\title{
Verbindung halten
}

(Post)kommunikation unter schwierigen Verhältnissen

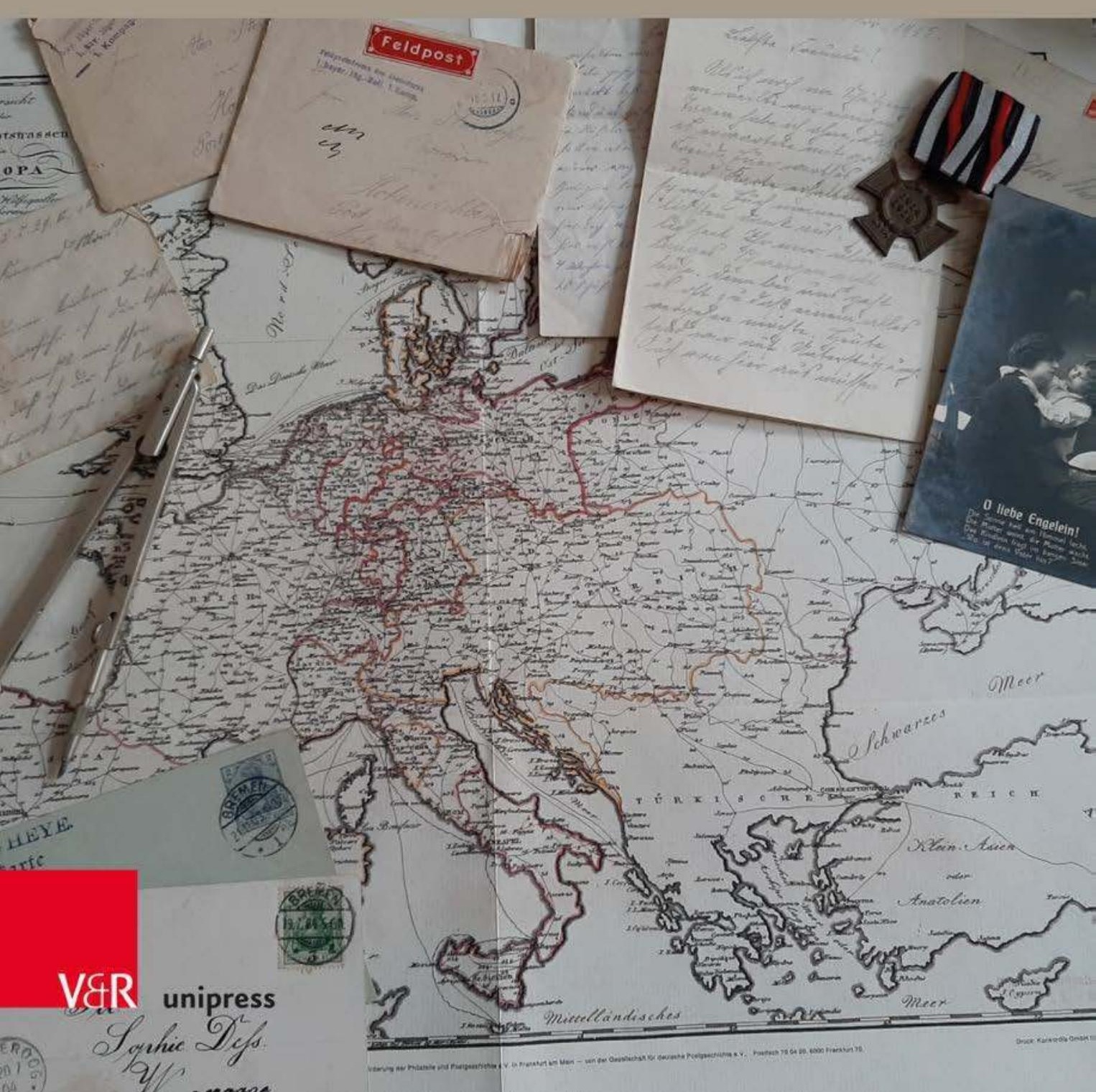


Post - Wert - Zeichen

Band 2

Herausgegeben von

Pierre Smolarski, René Smolarski und Silke Vetter-Schultheiß 


\section{René Smolarski (Hg.)}

\section{Verbindung halten}

\section{(Post)kommunikation unter schwierigen Verhältnissen}

Mit 62 Abbildungen

V\&R unipress 
Bibliografische Information der Deutschen Nationalbibliothek Die Deutsche Nationalbibliothek verzeichnet diese Publikation in der Deutschen Nationalbibliografie; detaillierte bibliografische Daten sind im Internet über https://dnb.de abrufbar.

Gedruckt mit freundlicher Unterstützung des Bundes Deutscher Philatelisten e.V., des Netzwerks für digitale Geisteswissenschaften und Citizen Science der Universität Erfurt und der Universitätsbibliothek Erfurt.

(c) 2021, Vandenhoeck \& Ruprecht GmbH \& Co. KG, Theaterstraße 13, D-37073 Göttingen Dieses Werk ist als Open-Access-Publikation im Sinne der Creative-Commons-Lizenz BY-NC-ND International 4.0 (»Namensnennung - Nicht kommerziell - Keine Bearbeitungen«) unter dem DOI 10.14220/9783737012287 abzurufen. Um eine Kopie dieser Lizenz zu sehen, besuchen Sie https://creativecommons.org/licenses/by-nc-nd/4.0/.

Jede Verwertung in anderen als den durch diese Lizenz zugelassenen Fällen bedarf der vorherigen schriftlichen Einwilligung des Verlages.

Umschlagabbildung: ๑ René Smolarski

Vandenhoeck \& Ruprecht Verlage | www.vandenhoeck-ruprecht-verlage.com

ISSN 2626-2134

ISBN 978-3-7370-1228-7 


\section{Inhalt}

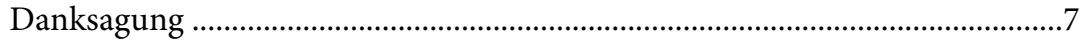

René Smolarski

Postgeschichte - Ein vernachlässigtes Gebiet historischer Forschung:

Zur Einführung .............................................................................................

Christoph Borbach

Gestörte Kommunikation. Die Geburt der Echoortung als Praxis der

Rohrpost - Eine Fußnote zur Mediengeschichte der Post

Christian Methfessel

Telegramme, Läufer, Brieftauben: Kommunikation in Zeiten

imperialer Kriege.

Stefan Geissler

Die Lloyd's List. Die Bedeutung des Postwesens für die frühneuzeitliche Marineversicherung.

Reiner Prass

Briefe aus Äthiopien und dem Sudan. Die Briefkommunikation zwischen europäischen Reisenden und dem Kartographen August

Petermann in Gotha, 1854-1880

Maksim Viktorovich Fomenko

Die Organisation und die Verwaltung der sowjetischen Militär-Feldpost

im Zweiten Weltkrieg

Heinz Wewer

Die Post der Häftlinge in Konzentrationslagern

Konstanze Soch

Ein »verpackter « Konflikt. Die persönliche und politische Dimension des Geschenkpaketverkehrs. 


\section{Amelie Dreecke}

Der Adel und die Ansichtskarte. Motivik im Dienste der Herrscher 209

Abbildungsnachweise 239 


\section{Danksagung}

Der vorliegende Band entstand im Nachgang der Tagung Klio \& Hermes: Post Geschichte - Kommunikation, die im November 2018 auf Initiative des leider unerwartet verstorbenen Romanisten und Philatelisten Reinhard Krüger im Museum für Kommunikation in Berlin durchgeführt worden ist. Die Heterogenität der umfangreichen Tagung machte es erforderlich, die Publikation der Beiträge in zwei inhaltlich unterschiedlich ausgerichteten Sammelbänden durchzuführen. Dieser Band konzentriert sich dabei auf die Aspekte der Postkommunikation und ihrer Bedeutung für die historische Forschung und wurde um weitere Beiträge aus diesem Untersuchungsfeld erweitert.

Das Entstehen dieses Bandes ist der fruchtbaren Kooperation vieler Beteiligter zu verdanken, denen hiermit ausdrücklich gedankt sein soll. Zu erwähnen wären hier nicht zuletzt die Teilnehmer und Teilnehmerinnen der Tagung, da die Veranstaltung ohne sie in einer so positiv empfundenen Atmosphäre nicht möglich gewesen wäre.

Finanziell ermöglicht haben diese Publikation der Bund Deutscher Philatelisten e.V., die Universitätsbibliothek Erfurt und das ebenfalls an der Universität Erfurt angesiedelte Netzwerk für digitale Geisteswissenschaften und Citizen Science.

Schließlich danke ich auch Juliane Wenke für das umfassende Lektorat und dem Verlag $V \& R$ unipress, und hier insbesondere Frau Marie-Carolin Vondracek, für die gute Betreuung bei der Drucklegung.

An dieser Stelle möchte ich auch auf die beiden Kartenabbildungen verweisen, die dem Band digital beigefügt sind und über die Webseite des Verlages heruntergeladen werden können (Vgl. http://vandenhoeck-ruprechtverlage.com/PWZ2 (unter Downloads)).

Erfurt, im Juli 2020 René Smolarski 
Open-Access-Publikation im Sinne der CC-Lizenz BY-NC-ND 4.0 (C) 2021, Vandenhoeck \& Ruprecht GmbH \& Co. KG, Göttingen ISBN Print: 9783847112280 - ISBN E-Lib: 9783737012287 


\section{Postgeschichte - Ein vernachlässigtes Gebiet historischer Forschung: Zur Einführung}

\section{Einleitung}

In seinem Portrait der Deutschen Bundespost, das 1982 unter dem Titel Damit wir in Verbindung bleiben ${ }^{1}$ erschien, stellte der damalige Bundespostminister Kurt Gscheidle (SPD) in Bezug auf eine der zentralen seinem Ressort unterstellten Institutionen, die Deutsche Bundespost, allgemeingültig fest:

»Die Post ist für die meisten Menschen unersetzlich, ja, lebensnotwendig. Dennoch ist sie kein Thema, das Menschen in ihrer Freizeit beschäftigt, wenn wir einmal von einigen Liebhabern und, natürlich, von den Philatelisten absehen, die in der Tat weit über die Briefmarken hinaus an der Post interessiert sind. $^{2}$

Ähnlich wie Gscheidle hier das Interesse der Gesellschaft an der Institution Post und ihren Aufgaben schildert, ließe sich auch das Interesse der Geschichtswissenschaft an der Post und der Postkommunikation beschreiben.

$\mathrm{Zu}$ einem ähnlichen Befund kam bereits 1984 auch der Nahost-Historiker Donald M. Reid, als er in einem Beitrag über den Symbolismus von Briefmarken als Quelle historischer Forschung konstatierte, dass zwar historische Untersuchungen nationaler Postinstitutionen existierten, diese jedoch nur von wenigen Historikern gelesen würden und auch in allgemeinen Übersichtwerken lediglich die Bedeutung von Dampfschiffen, Lokomotiven und Telegraphen für die Postrevolution im 19. Jahrhundert hervorgehoben wird. ${ }^{3}$ Reid

1 Der Titel des hier vorliegenden Sammelbandes orientiert sich an diesem von Gscheidle gewählten Titel.

2 Kurt Gscheidle: Damit wir in Verbindung bleiben. Portrait der Deutschen Bundespost, Stuttgart 1982, S. 11-12.

3 Reid führt hier beispielhaft die folgenden an: Eric J. Hobsbawm: Age of Capital 1848-1875, New York 1976 (dt. Eric J. Hobsbawm: Die Blütezeit des Kapitals. Eine Kulturgeschichte der Jahre 1848-1875, Zürich 1979) und John Patrick Tuer Bury: New Cambridge Modern History. The zenith of European power 1830-70, Cambridge 1967. Beispielhaft sei hier aus der neueren Literatur aber auch verwiesen auf: Jürgen Osterhammel: Die Verwandlung der Welt. Eine Ge- 
betonte in diesem Zusammenhang, dass die Telegraphenleitungen es seit etwa 1872 ermöglichten, diplomatische Depeschen, Neuigkeiten und Börsenkurse in hoher Geschwindigkeit von London nach New York, Bombay, Tokyo oder Adelaide zu übermitteln, angesichts dieses weltweiten Telegraphennetzes jedoch die Bedeutung des einfachen Postverkehrs, der zur gleichen Zeit ebenfalls ermöglichte, eine Nachricht in Form eines Briefes an nahezu jeden Ort in der Welt in wenigen Tagen oder Wochen statt Monaten zu übermitteln, vielfach übersehen würde. ${ }^{4}$

Eine signifikante Intensivierung der Postgeschichtsforschung setzte jedoch trotz dieses Aufrufs nicht ein. Vielmehr entstammt ein Großteil der historischen Analysen der Postgeschichte, wenn auch nicht alle, ${ }^{5}$ bis heute aus dem Umfeld der Philatelie, die in der Regel außeruniversitär organisiert ist und sich in verschieden Arbeitsgruppen den Fragen der postalischen Kommunikation angenommen hat und weiterhin annimmt, oder aus postgeschichtlich interessierten bürgerwissenschaftlichen Kreisen. ${ }^{6}$ In der universitären Geschichtswissenschaft sind sowohl Philatelie als auch Postgeschichte hingegen eher randständige Themen. ${ }^{7}$

schichte des 19. Jahrhunderts, München 2013, S. 74-76 und Thomas Nipperdey: Deutsche Geschichte 1866-1918. Arbeitswelt und Bürgergeist, München 1990, S. 260-263.

4 Vgl. Donald. M. Reid: The Symbolism of Postage Stamps: A Source for the Historian, in: Journal of Contemporary History 19 (1984) 2, S. 223-249, hier S. 225.

5 Für das späte 19. und frühe 20. Jahrhundert sei hier unter anderem auf das entsprechende Kapitel Siegfried Weichleins Habilitationsschrift verwiesen. Vgl. Siegfried Weichlein: Nation und Region. Integrationsprozesse im Bismarckreich, 2. Auflage, Düsseldorf 2006, S. 105-190. $\mathrm{Zu}$ verschiedenen Aspekten der Postgeschichte siehe auch Josef Foschepoth: Überwachtes Deutschland. Post- und Telefonüberwachung in der alten Bundesrepublik, Göttingen 2013; Wolfgang Behringer: Im Zeichen des Merkur, Reichspost und Kommunikationsrevolution in der Frühen Neuzeit, Göttingen 2013; Ders.: Thurn und Taxis. Die Geschichte ihrer Post und ihrer Unternehmen, München u. a. 1990; Wolfgang Lotz, Gerd R. Ueberschär: Die Deutsche Reichspost 1933-1945. Eine politische Verwaltungsgeschichte. 2 Bände. Berlin 1999; Karin Amtmann: Post und Politik in Bayern von 1808-1850. Der Weg der königlich-bayrischen Staatspost in den Deutsch-Österreichischen Postverein, München 2006 und Andreas Benz: Integration von Infrastrukturen in Europa im historischen Vergleich, Bd. 3: Post, Baden-Baden 2013.

6 Neben unzähligen heimatgeschichtlich motivierten "Postgeschichten« seien hier wenige Publikationen beispielhaft angeführt. So unter anderem Jürgen Ruszkowski: Post \& Seefahrt. Die Rolle der Seefahrt in der Postgeschichte, Berlin 2019; Wolfgang Reifferscheid: Rohrpost/Stadtrohrpost Berlin. Die Rohrpost in Berlin bis zum Ende des Dritten Reichs, Berlin 2018; Horst Diederichs: Die Umgestaltung des deutschen Postwesens zwischen der Französischen Revolution (1792) und dem Wiener Kongreß (1814/15), Bietigheim-Bissingen 2016 und Janusz Mozdzan: Postgeschichte des Konzentrationslagers Lublin - Majdanek: über das Lager, Briefe und Menschen, Manching 2010.

7 Ein Blick in die Publikationen des letzten Jahres zeigt jedoch, dass die Wissenschaft zumindest die Philatelie selbst stärker in den Blick genommen hat. Genannt seien hier neben dem ersten Band dieser Reihe (Pierre Smolarski, René Smolarski, Silke Vetter Schultheiß (Hrsg): Gezähnte 
Dies ist umso erstaunlicher, als der Post in der Kommunikationsgeschichte und damit in der Gesellschaftsgeschichte eine besondere Bedeutung zukommt. Dies stellte schon Gscheidle fest, als er diesbezüglich schrieb, dass »[a]lle erwachsenen Bürger [...] nahezu täglich mit der Post zu tun [hätten]«, die damit einhergehende »Wechselbeziehung [jedoch] in der Regel nur dann [registriert würde], wenn sie gestört ist und nicht funktioniert. ${ }^{8}{ }^{8}$ Wenn die Störung aber beseitigt wäre und sich die Lage normalisiert hätte, würde das Interesse sofort wieder absinken. Die Post sei eben, so der ehemalige Postminister, etwas Selbstverständliches.

\section{Konzeption des Bandes}

Dass die Post und ihre Geschichte nichts Selbstverständliches ist, versucht dieser Band aus einer Mehrzahl von Perspektiven zu verdeutlichen. Hervorgegangen sind die Idee zu diesem Band sowie ein Teil der hier versammelten Beiträge aus der Tagung Klio \& Hermes: Post - Geschichte - Kommunikation, die im November 2018 auf Initiative des leider bereits verstorbenen Romanisten und Philatelisten Reinhard Krüger im Museum für Kommunikation in Berlin durchgeführt worden ist. Aufgrund der Größe der Veranstaltung und der Heterogenität der dort behandelten Themen erschien es jedoch notwendig, die schriftlichen Beiträge der Veranstaltung in zwei thematisch getrennte Sammelbände zu bringen, von denen sich der hier vorliegende Band dem Thema Postkommunikation widmet. Im Fokus stehen dabei vor allem die von Gscheidle erwähnten Störungen bzw. Unregelmäßigkeiten im postalischen Verkehr, deren Ursachen sowohl auf politischen als auch technischinfrastrukturellen Gegebenheiten basieren konnten. Der Begriff der Störung ist dabei breit zu verstehen und kann eine konkrete und in der Regel punktuelle technische Störung ${ }^{9}$ oder auch durch Zeitverzögerungen provozierte bzw. begünstigte Veränderungen im Kommunikationsprozess ${ }^{10}$ meinen. Der Begriff soll an dieser Stelle aber auch die Auswirkungen politischer und gesellschaftlicher Rahmenbedingungen umfassen, die nicht immer zu einer tatsächlichen

Geschichte. Die Briefmarke als historische Quelle, Göttingen 2019) auch die folgenden Sammelbände: Dirk Naguschewski, Detlev Schöttker (Hrsg.): Philatelie als Kulturwissenschaft. Weltaneignung im Miniaturformat, Berlin 2019 und Achim Thomas Hack, Klaus Ries (Hrsg.): Geschichte zum Aufkleben. Historische Ereignisse im Spiegel deutscher Briefmarken, Stuttgart 2020.

8 Gscheidle 1982, S. 12.

9 Siehe hierzu den Beitrag von Christoph Borbach in diesem Band.

10 Siehe hierzu die Beiträge von Christian Methfessel, Stefan Geissler und Reiner Prass in diesem Band. 
Störung der postalischen Kommunikation führten, sondern diese unter Umständen nur - wenn auch nicht selten in erheblicher Art und Weise - erschwerten ${ }^{11}$ oder durch gesellschaftliche Normen und Erwartungen in ihrer Ausprägung veränderten. ${ }^{12}$

Der Band unternimmt damit den Versuch, in Anknüpfung an die bereits erschienenen Ergebnisse der vorhergehenden Tagung vom Oktober $2017^{13}$ und in Fortführung des dort begonnenen bürgerwissenschaftlichen Dialogs ${ }^{14}$ zwischen universitärer Wissenschaft und außeruniversitärer Philatelie, die Bedeutung philatelistischer und postgeschichtlicher Untersuchungen für die verschiedensten Bereiche der Geschichtswissenschaft auszuloten und damit Impulse für eine grundwissenschaftlich verstandene Philatelie zu legen. ${ }^{15}$

\section{Die Beiträge}

Christoph Borbach widmet sich in seinem Beitrag einer ganz spezifischen, technisch bedingten Form der Störung postalischer Kommunikation, nämlich, der Ortung steckengebliebener Büchsen in den Poströhren des 19. Jahrhunderts. Gemeint ist hier das von dem französischen Ingenieur Charles Bontemps entwickelte Ortungsverfahren, mit dem auf Basis der gemessenen Schallgeschwindigkeit, die Störungen in den Rohrpostanlagen gefunden und somit effizient beseitigt werden konnten. Borbach beschreibt damit nicht nur einen für den Betrieb der Pariser Röhrenpost relevanten Aspekt der Kanalwartung und -reparatur, sondern zeigt zudem die Bedeutung des damit verbundenen methodischen Vorgehens für die Technikgeschichte der Akustik und unter-

11 Siehe hierzu die Beiträge von Maksim Fomenko, Heinz Wewer und Konstanz Soch in diesem Band.

12 Siehe hierzu den Beitrag von Amelie Dreecke in diesem Band.

13 Vgl. Smolarski, Smolarski, Vetter-Schultheiß 2019.

14 Zur Frage der Bürgerwissenschaften, die unter dem Schlagwort citizen science auch in den Geisteswissenschaften vermehrt Bedeutung erlangt und sich insbesondere auch an Sammlerund Hobbygemeinschaften richtet siehe unter anderem Gerhard Ott: Hobbys. Private Quellen der Bürgerwissenschaft, in: Peter Finke (Hrsg.): Freie Bürger - Freie Forschung. Die Wissenschaft verlässt den Elfenbeinturm, München 2015, S. 70-74, und die verschiedenen Beiträge in Kristin Oswald, René Smolarski. Bürger - Künste - Wissenschaft. Citizen Science in Kultur und Geisteswissenschaften, Gutenberg 2016.

15 Auseinandersetzungen darüber, ob die Philatelie in die Reihe der historischen Hilfs- oder Grundwissenschaften gehört, finden sich unter anderem bei Joachim Helbig: Ist Philatelie eine Hilfswissenschaft?, in: Postgeschichte - Historie Postale - Storia Postale 82 (2000), S. 19-28, und Pierre Smolarski, René Smolarski: Wissenschaftliches Stiefkind und amateurhafte Liebhaberei: Ein designrhetorischer Zugang zur Philatelie als historischer Grundwissenschaft, in: Étienne Doublier, Daniela Schulz, Dominik Trump: Die Historischen Grundwissenschaften im Spannungsfeld zwischen Interdisziplinarität und Profilierung, Köln 2020, S. 95-119. 
streicht damit vor allem dessen medienhistorische Signifikanz und die Verwobenheit von Post- und Mediengeschichte.

Ebenso wie bei Borbach stehen auch im Beitrag von Christian Methfessel Verzögerungen der postalischen Kommunikation im Mittelpunkt der Betrachtung. Anders als im ersten, stellen diese delays jedoch im zweiten Beitrag kein inakzeptables technisches Übel dar, das es schnellstmöglich zu beseitigen galt, sondern im Kontext militärischer Auseinandersetzungen im Zeitalter imperialer Kriege vielmehr eine strukturell bedingte Ursache für Verschiebungen und Veränderungen in der öffentlichen Wahrnehmung dieser Konflikte. Methfessel zeigt anhand der Presseberichterstattung über den sogenannten `Boxerkrieg` in China und den >Hüttensteuerkrieg` in Sierra Leone auf, wie die zeitgenössischen Kommunikationswege die mediale Berichterstattung über imperiale Krisen und damit auch das Bild der Öffentlichkeit über diese und die damit direkt zusammenhängenden politischen Handlungsspielräume beeinflussten. ${ }^{16}$ Der Autor sieht in der Reflexion der zeitgenössischen Kommunikationswege einen unerlässlichen Baustein für das Verständnis der in Europa über die verschiedenen, weltweiten Militäreinsätze geführten Kontroversen im Zeitalter des Imperialismus. Dies vor allem deswegen, da der zurückzulegende Kommunikationsweg und der übermittelbare Umfang einer Nachricht die mit der Botschaft transportierten Deutungsmuster und damit auch das von der europäischen Presse vermittelte Bild des Geschehens maßgeblich beeinflussten.

Einem ganz anderen Aspekt der Postkommunikation, nämlich ihrer Bedeutung für das frühneuzeitliche Marineversicherungswesen geht Stefan Geissler in seinem Beitrag über die Entstehung der Lloyd's List, einer hochspezialisierten Londoner Wirtschaftszeitung, die vor allem auch über Standort und $\mathrm{Zu}$ stand von Hochseeschiffen informierte, nach. ${ }^{17}$ Um diese Informationen zeitnah zu erhalten war man bei Lloyd's of London Ende des 18. Jahrhunderts nicht zuletzt auch darum bemüht, einen engen Kontakt zur Post, die zeitgemäß quasi das wichtigste Netzwerk der Informationsübermittlung darstellte, zu unterhalten. Folgerichtig liegt das besondere Augenmerk von Geissler daher auf der aktiven Einflussnahme des Versicherungskonzerns auf die regulären

16 Zur Bedeutung der Medienberichterstattung im Kontext imperialer Auseinandersetzungen siehe vor allem die Dissertationsschrift von Methfessel, die diesem Beitrag zugrunde liegt: Christian Methfessel: Kontroverse Gewalt. Die imperiale Expansion in der englischen und deutschen Presse vor dem zweiten Weltkrieg, Köln/Weimar 2019.

17 Der Bereich der Schiffspost stellt in der Geschichtswissenschaft in vielen Bereichen ein Desiderat dar. Vorhandene Untersuchungen und Beschreibungen, die sich in der Regel auf eine konkrete Region beziehen, stammen in erster Linie aus dem Umfeld philatelistisch oder posthistorisch Interessierter. So zum Beispiel Richard Frick: Schiffspost im Nordsee- und Ostseeraum mit Anhang Deutsche Schiffs- und Seepost, Hannover 1981 und Thomas Keller: Die Verkehrsentwicklung und die Organisation der Schiffspost auf dem Zürichsee im 19. Jahrhundert, Selbstverlag 1994. 
postalischen Arbeitsabläufe, um sich selbst einen Geschwindigkeits- und damit Wettbewerbsvorteil gegenüber der Konkurrenz zu sichern. Eng damit verbunden war ein Aufbau eines weltweiten Agentennetzwerkes, durch welches man zum einen über Ansprechpartner für die Kunden vor Ort verfügte und zum anderen auch Einfluss auf politische Entscheidungen zu gewinnen suchte. Die engen Verbindungen von Lloyd's zum Postwesen beschränkten sich daher nicht allein auf die Kontakte zur britischen Post, sondern umfassten nach und nach auch immer mehr Poststellen weltweit.

Die internationale Postkommunikation steht auch im Zentrum des von Reiner Prass verfassten Beitrags über den Briefverkehr europäischer Forschungsreisender in Äthiopien und dem Sudan mit dem im Verlagshaus Justus Perthes in Gotha arbeitenden Kartographen August Petermann. Anhand der in der Sammlung Perthes ${ }^{18}$ überlieferten Briefkorrespondenz einiger auf dem Gebiet des heutigen Äthiopien und Sudan agierender Forschungsreisender, wie Gustav Nachtigal, Georg Rohlfs und Theodor von Heuglin, ermittelt Prass nicht nur die Brieflaufzeiten von verschiedenen afrikanischen Orten in das thüringische Gotha in der zweiten Hälfte des 19. Jahrhunderts, sondern rekonstruiert auch die Besonderheiten und spezifischen Charakteristiken der Kommunikationswege in diesem Teil Afrikas. Gerade diese Besonderheiten, die nicht selten langwierige Verzögerungen in der Postübermittlung oder gar den Verlust ganzer Postsendungen mit sich brachten, stellten eine besondere Herausforderung für die Arbeit der europäischen Forschungsreisenden dar, die sich mit ihren Briefen und Postsendungen, so Prass, direkt am Aufbau eines über kulturelle Grenzen hinweg reichenden Kommunikationsraums beteiligten. Die Erforschung der konkreten Ausformungen dieses Kommunikationsraums, der sich durch technische Neuerungen und infrastrukturelle Maßnahmen, wie den Bau des Suezkanals, die Inbetriebnahme von Eisenbahnverbindungen oder der Errichtung eines weltumspannenden Telegraphennetzwerks in der zweiten Hälfte des 19. Jahrhunderts zunehmend verdichtete, steht, wie der Autor betont, jedoch noch am Anfang.

Während Reiner Prass sich in seinem Beitrag der Organisation von postalischer Kommunikation auf der persönlichen Ebene aus dem Blick einzelner Entdeckungsreisender im 19. Jahrhundert widmete, nimmt Maksim Fomenko den Organisationsaspekt aus einem anderen Blickwinkel in Betracht. In seinem Beitrag beschreibt der Autor die Entwicklung der Verwaltung und Organisation der sowjetischen Feldpost im Verlauf des Zweiten Weltkriegs und behandelt damit ein Thema, das in der vergleichsweise umfangreichen deutschspra-

18 Siehe hierzu unter anderem Kathrin Paasch, Petra Weigel, Tan Sergej: Die Sammlung Perthes Gotha, Erfurt 2010. 
chigen Forschungsliteratur zur Feldpost ${ }^{19}$ - insbesondere des Ersten Weltkriegs - bislang wenig Beachtung fand..$^{20}$ Ausgehend von der Feststellung, dass die Feldpost und der von ihr hauptverantwortlich sicherzustellende Kommunikationsweg zwischen Front und Heimat nicht zuletzt auch wesentlich zur Erhaltung der Moral der kämpfenden Truppe beitrug, ${ }^{21}$ erläutert Fomenko, wie vor allem der Volkskommissar der Kommunikation Ivan Terentyevich Peresypkin die anfänglichen Probleme überwand und die Funktionsfähigkeit der sowjetischen Feld- und Marinepost verbesserte. Ein wesentlicher Kernpunkt dieser Verbesserungen beruhte auf der Überarbeitung der Adressangaben, die auf der einen Seite die postalische Zustellung durch möglichst genaue Angaben sicherstellen, auf der anderen Seite jedoch dem Gegner, im Falle einer Erbeutung der Post, so wenig wie möglich Angaben über die Struktur und räumliche Verteilung der sowjetischen Streitkräfte liefern sollten. Letztlich übernahm man hierbei sogar das fünfstellige Postleitzahlensystem der Wehrmacht, um die Übermittlung der Post und damit auch der berühmten Dreiecksbriefe von der Heimat an die Front und vice versa sicherzustellen.

Heinz Wewer untersucht in seinem Beitrag die Möglichkeiten postalischer Kommunikation in den nationalsozialistischen Konzentrationslagern zwischen 1933 und 1945. Er betont hierbei die Bedeutung, die die ein- und ausgehende Post für die Häftlinge hatte, da diese doch, von sehr wenigen Ausnahmen abgesehen, die einzige verbleibende Verbindung zur Welt außerhalb des Lagers darstellte. Umso mehr nutzten die Lageraufseher daher auch Restriktionen beim Postverkehr als Bestrafungsmaßnahme - unter anderem bei Zuwiderhandlung gegen ein umfangreiches, undurchsichtiges und, wie der Autor hervorhebt, den einzelnen Häftlingen zumeist unbekanntes Regelwerk für die Postkommunikation, das zudem von Lager zu Lager verschieden war und sich des Weiteren auch mit der Zeit wandelte. Anhand eindrucksvoller Beispiele zeichnet Wewer die Entwicklungsgeschichte der Postbestimmungen in den Konzentrationslagern nach und geht dabei auch auf die vielen Sonderbestimmungen und Abweichungen für die verschiedenen Häftlingsgruppen ein.

19 Siehe hierzu unter anderem den aus einer Tagung im Museum für Kommunikation Berlin im September 2010 hervorgegangenen Sammelband: Veit Didczuneit, Jens Ebert, Thomas Jander (Hrsg.): Schreiben im Krieg - Schreiben vom Krieg. Feldpost im Zeitalter der Weltkriege, Essen 2011.

20 Einen Überblick über die organisatorische Entwicklung der sowjetischen Feldpost gibt unter anderem Elke Scherstjanoi: »Wir sind in der Höhle der Bestie." Die Briefkommunikation mit der Heimat über ihre Erlebnisse in Deutschland, in: Dies. (Hrsg.): Rotarmisten schreiben aus Deutschland: Briefe von der Front (1945) und historische Analysen, München 2004, S. 194-228.

21 Siehe hierzu unter auch Clemens Schwender: Feldpost als Medium sozialer Kommunikation, in: Veit Didczuneit, Jens Ebert, Thomas Jander (Hrsg.): Schreiben im Krieg - Schreiben vom Krieg. Feldpost im Zeitalter der Weltkriege, Essen 2011, S. 127-138. 
Mit dem deutsch-deutschen Päckchen- und Paketverkehr zur Zeit des Kalten Krieges stellt Konstanze Soch in ihrem Beitrag einen wichtigen Aspekt der deutschen Geschichte des 20. Jahrhunderts in den Mittelpunkt ihrer Untersuchung, der über mehrere Generationen hinweg eine wenngleich, wie die Autorin deutlich macht, für jede Generation auf jeweils eigene Weise prägende, Wirkung hatte. ${ }^{22}$ Soch richtet dabei ihren Blick nicht allein auf das berühmte Westpaket, sondern auch auf die Geschenksendungen, die die innerdeutsche Grenze in die entgegengesetzte Richtung passierten. Ausgehend von einem Vergleich der generationellen Besonderheiten dieses von beiden politischen Systemen in unterschiedlicher Art und Weise gewollten innerdeutschen Austausches, der sich nicht nur in den Inhalten der Pakete, sondern auch in den an sie geknüpften Erwartungen äußert, beschreibt die Autorin die verschiedenen Stadien des Paketverkehrs während des Kalten Krieges und darüber hinaus. Im Mittelpunkt stehen hierbei insbesondere die Paketkontrollen auf beiden Seiten der Mauer und die damit verbundenen Diebstähle in der DDR. Soch gibt zudem einen vergleichenden Einblick in den Geschenkpaketverkehr der beiden deutschen Staaten nach Polen.

Amelie Dreecke untersucht in ihrem Beitrag über die Ansichtskartenmotivik im Dienste des deutschen Adels einen besonderen Aspekt der Postkommunikation, bei dem es vor allem um die Erfüllung und Setzung von gesellschaftlichen Normen und die Präsentation des Herrscherhauses auf einem der weit verbreitetsten philatelistischen Medien geht. ${ }^{23}$ Dreecke geht dabei anhand ausgewählter Beispiele den Fragen nach, in welcher Art und Weise sich der Adel auf Ansichtskarten präsentierte beziehungsweise präsentiert wurde, welche Wertvorstellungen mit diesem Massenmedium transportiert und welche

22 Siehe hierzu auch die dem Beitrag zugrunde liegende Dissertation: Konstanze Soch: Eine große Freude? Der innerdeutsche Paketverkehr im Kalten Krieg (1949-1989), Frankfurt am Main/ New York 2018.

23 Post- und Ansichtspostkarten - wobei letztere auch eine Form der Postkarte ist, die jedoch in der Regel nicht amtlich verausgabt wurde und mit einem Bilddruck oder Foto auf der Rückseite versehen ist (Vgl. Ulrich Häger: Kleines Lexikon der Philatelie, Gütersloh 1977, S. 22) -, sind eine der wenigen philatelistischen Quellen, denen sich die universitäre Geschichtswissenschaft verschiedentlich, wenn auch in der Regel mit Blick auf die Bildseite, zugewandt hat. Zu nennen wären hier neben allgemeinen Übersichten zur Mediengeschichte (so zum Beispiel Werner Faulstich: Medienwandel im Industrie- und Massenzeitalter (1830-1900), Göttingen 2004, S. 167 ff.) unter anderem Hinnerk Onken: Ambivalente Bilder. Fotografien und Bildpostkarten aus Südamerika im Deutschen Reich (1880-1930), Bielefeld 2019 und Helmut Gold, Georg Heuberger: Abgestempelt. Judenfeindliche Postkarten, Heidelberg 1999. Einen Blick auf die verbale Funktion der Postkarte richtet unter anderem Anett Holzheid: Das Medium Postkarte: Eine sprachwissenschaftliche und mediengeschichtliche Studie, Berlin 2011. In den Veröffentlichungen der außeruniversitären Philatelie nehmen Untersuchungen zu Ansichts- und Bildpostkarten ebenfalls einen besonderen Platz ein. Zu nennen wären hier vor allem die umfangreichen Arbeiten des Lehrers und passionierten Sammlers Otto May. 
Formen zeitgenössischer Propaganda durch die entsprechende Präsentationsweise angesprochen wurden. Zudem fragt die Autorin danach, welche stilistischen Unterschiede zwischen den Ansichtskarten des späten 19. und frühen 20. Jahrhunderts und den klassischen Kunstwerken - vor allem der Malerei zu identifizieren sind. Thematischer Ausgangspunkt von Dreeckes Untersuchung sind die verschiedenen Schritte des damals üblichen und in der Regel geschlechtsspezifischen Lebenswegs und Werdegangs einer beziehungsweise eines Adeligen um die Jahrhundertwende, den sie auf der Grundlage diverser Ansichtskarten illustriert und durch historische Vor- als auch Rückgriffe erläutert. Im Zentrum stehen daher vor allem die adelige Hochzeit, die Sicherung der Nachfolge, die militärische Laufbahn der männlichen Adeligen und nicht zuletzt der Tod und die posthume Glorifizierung.

\section{Literatur}

Karin Amtmann: Post und Politik in Bayern von 1808-1850. Der Weg der königlichbayrischen Staatspost in den Deutsch-Österreichischen Postverein, München 2006.

Wolfgang Behringer: Im Zeichen des Merkur, Reichspost und Kommunikationsrevolution in der Frühen Neuzeit, Göttingen 2013.

Wolfgang Behringer: Thurn und Taxis. Die Geschichte ihrer Post und ihrer Unternehmen. Piper, München u. a. 1990.

Andreas Benz: Integration von Infrastrukturen in Europa im historischen Vergleich, Bd. 3: Post, Baden-Baden 2013.

Veit Didczuneit, Jens Ebert, Thomas Jander (Hrsg.): Schreiben im Krieg - Schreiben vom Krieg. Feldpost im Zeitalter der Weltkriege, Essen 2011.

Horst Diederichs: Die Umgestaltung des deutschen Postwesens zwischen der Französischen Revolution (1792) und dem Wiener Kongreß (1814/15), Bietigheim-Bissingen 2016.

Werner Faulstich: Medienwandel im Industrie- und Massenzeitalter (1830-1900), Göttingen 2004.

Josef Foschepoth: Überwachtes Deutschland. Post- und Telefonüberwachung in der alten Bundesrepublik, Göttingen 2013.

Richard Frick: Schiffspost im Nordsee- und Ostseeraum mit Anhang Deutsche Schiffs- und Seepost, Hannover 1981.

Helmut Gold, Georg Heuberger: Abgestempelt. Judenfeindliche Postkarten, Heidelberg 1999.

Kurt Gscheidle: Damit wir in Verbindung bleiben. Portrait der Deutschen Bundespost, Stuttgart 1982.

Ulrich Häger: Kleines Lexikon der Philatelie, Gütersloh 1977, S. 22.

Joachim Helbig: Ist Philatelie eine Hilfswissenschaft?, in: Postgeschichte - Historie Postale Storia Postale 82 (2000), S. 19-28.

Eric J. Hobsbawm: Age of Capital 1848-1875, New York 1976. 
Eric J. Hobsbawm: Die Blütezeit des Kapitals. Eine Kulturgeschichte der Jahre 1848-1875, Zürich 1979.

Anett Holzheid: Das Medium Postkarte: Eine sprachwissenschaftliche und mediengeschichtliche Studie, Berlin 2011.

Achim Thomas Hack, Klaus Ries (Hrsg.): Geschichte zum Aufkleben. Historische Ereignisse im Spiegel deutscher Briefmarken, Stuttgart 2020.

Thomas Keller: Die Verkehrsentwicklung und die Organisation der Schiffspost auf dem Zürichsee im 19. Jahrhundert, Selbstverlag 1994.

Wolfgang Lotz, Gerd R. Ueberschär: Die Deutsche Reichspost 1933-1945. Eine politische Verwaltungsgeschichte. 2 Bände. Berlin 1999.

Christian Methfessel: Kontroverse Gewalt. Die imperiale Expansion in der englischen und deutschen Presse vor dem zweiten Weltkrieg, Köln/Weimar 2019.

Janusz Mozdzan: Postgeschichte des Konzentrationslagers Lublin - Majdanek: über das Lager, Briefe und Menschen, Manching 2010.

Dirk Naguschewski, Detlev Schöttker (Hrsg.): Philatelie als Kulturwissenschaft. Weltaneignung im Miniaturformat, Berlin 2019.

Thomas Nipperdey: Deutsche Geschichte 1866-1918. Arbeitswelt und Bürgergeist, München 1990.

Jürgen Osterhammel: Die Verwandlung der Welt. Eine Geschichte des 19. Jahrhunderts, München 2013, S. 74-76.

Kristin Oswald, René Smolarski. Bürger - Künste - Wissenschaft. Citizen Science in Kultur und Geisteswissenschaften, Gutenberg 2016.

Gerhard Ott: Hobbys. Private Quellen der Bürgerwissenschaft, in: Peter Finke (Hrsg.): Freie Bürger - Freie Forschung. Die Wissenschaft verlässt den Elfenbeinturm, München 2015, S. 70-74.

Kathrin Paasch, Petra Weigel, Tan Sergej: Die Sammlung Perthes Gotha, Erfurt 2010.

Donald. M. Reid: The Symbolism of Postage Stamps: A Source fort he Historian, in: Journal of Contemporary History 19 (1984) 2, S. 223-249.

Wolfgang Reifferscheid: Rohrpost/Stadtrohrpost Berlin. Die Rohrpost in Berlin bis zum Ende des Dritten Reichs, Berlin 2018.

Jürgen Ruszkowski: Post \& Seefahrt. Die Rolle der Seefahrt in der Postgeschichte, Berlin 2019.

Elke Scherstjanoi: „Wir sind in der Höhle der Bestie.« Die Briefkommunikation mit der Heimat über ihre Erlebnisse in Deutschland, in: Dies. (Hrsg.): Rotarmisten schreiben aus Deutschland: Briefe von der Front (1945) und historische Analysen, München 2004, S. 194-228.

Clemens Schwender: Feldpost als Medium sozialer Kommunikation, in: Veit Didczuneit, Jens Ebert, Thomas Jander (Hrsg.): Schreiben im Krieg - Schreiben vom Krieg. Feldpost im Zeitalter der Weltkriege, Essen 2011, S. 127-138.

Pierre Smolarski, René Smolarski: Wissenschaftliches Stiefkind und amateurhafte Liebhaberei: Ein designrhetorischer Zugang zur Philatelie als historischer Grundwissenschaft, in: Étienne Doublier, Daniela Schulz, Dominik Trump: Die Historischen Grundwissenschaften im Spannungsfeld zwischen Interdisziplinarität und Profilierung, Köln 2020, S. 95119. 
Pierre Smolarski, René Smolarski, Silke Vetter Schultheiß: Gezähnte Geschichte. Die Briefmarke als historische Quelle, Göttingen 2019.

John Patrick Tuer Bury: New Cambridge Modern History. The zenith of European power 1830-70, Cambridge 1967.

Siegfried Weichlein: Nation und Region. Integrationsprozesse im Bismarckreich, 2. Auflage, Düsseldorf 2006. 
Open-Access-Publikation im Sinne der CC-Lizenz BY-NC-ND 4.0 (C) 2021, Vandenhoeck \& Ruprecht GmbH \& Co. KG, Göttingen ISBN Print: 9783847112280 - ISBN E-Lib: 9783737012287 


\section{Christoph Borbach}

\section{Gestörte Kommunikation. Die Geburt der Echoortung als Praxis der Rohrpost - Eine Fußnote zur Mediengeschichte der Post}

\section{Medien- und Postgeschichte}

Auf die Relevanz der Postgeschichte für Fragen der Medienwissenschaft oder genauer: der Mediengeschichte und Medienepistemologie ist schon mehrfach hingewiesen worden. Dafür ließen sich verschiedene Beispiele geben, von denen ich im Folgenden nur zwei anführen will, nämlich die Möglichkeit der Bedingung der Etablierung von Autorschaft im 18. und 19. Jahrhundert und zum zweiten das Konzeptionalisieren von Hausnummern als postalische Adressen.

Der Medienhistoriker Bernhard Siegert arbeitete in Relais. Geschicke der Literatur als Epoche der Post die mediengeschichtliche und -theoretische Bedeutung heraus, die vor allem Medientechniken der Übertragung für literarische Werke und die Autorität eines Autors vornehmlich im 18. und 19. Jahrhundert innehatten. ${ }^{1}$ Er legte dar, dass postalische Übertragungstechniken die Bedingung für die Distribution und Zirkulation literarischer Werke und damit zugleich die Grundlage dafür waren, dass es den Autor als Autorität überhaupt geben kann. Dies gilt für den Download eines digitalen E-Books und - wie im Falle des Untersuchungshorizonts Siegerts - postalische Übertragungstechniken physischer, das heißt gedruckter Bücher. Daher ist die Geschichte der Literatur für Siegert zwischen dem 17. und 19. Jahrhundert wesentlich eine Geschichte der Post als ihrer Bedingung, fiel doch die Übertragung von Dingen und Nachrichten, aber auch Personen in ihren Kompetenzbereich. Dazu waren komplexe Postsysteme notwendig, die menschliche Akteure (institutionelle Boten, Postbeamte), aber auch Infrastrukturen (physische Stationen, Bahnpostwagen, Bahnschienen) und nicht zuletzt nicht-menschliche Akteure (Bü-

1 Bernhard Siegert: Relais. Geschicke der Literatur als Epoche der Post, Berlin 1993. 
cher, Postpferde) systematisch miteinander in Beziehung setzten, um überhaupt erst die Verbreitung später kanonisch gewordener Literatur zu gewährleisten. ${ }^{2}$ Denn ohne die Post als Distributionssystem wäre auch der Autor Goethe nie zur Autorität geworden. Nicht umhin meint der terminus technicus Relais nicht ausschließlich ein elektrisches Schaltelement, sondern geht etymologisch auf einen genuinen Postbegriff zurück: Das Relais beziehungsweise die Relaisstation war der Post-institutionelle Ort, an welchem Postkutscher ihre müden und erschöpften Pferde gegen `frische` und erholte, mithin schnellere Pferde auswechseln konnten. Im Relais als vortechnischer Einrichtung schaltete also nicht ein Schalter, sondern wechselte ein Postkutscher auf ein frisches Pferd um.

Posthistorisch relevant für die Medienwissenschaft wäre beispielsweise auch die produktive Zweckentfremdung staatshoheitlicher Rechteeinforderung, die in dem kulminierte, was sich unter dem Begriff der Adresse firmiert und erst später der Identifikation von Sendungs-Zielen diente. Zweck der Häusernummerierung als originärer Praktik der objektiven Adressierbarkeit auf Basis alphanumerischer Codes war es nämlich nicht, Fremden oder Einheimischen die Orientierung oder einem Postbeamten eine Briefzustellung zu ermöglichen, sondern die Hausnummerierung diente zunächst ausschließlich staatlichen Zielen wie der Besteuerung, der Brandschutzversicherung oder der Militäreinquartierung und -rekrutierung, wie Elena Fingerhut zeigte. ${ }^{3}$ Erst postalische Praktiken waren es, die (staatlich vergebene) Hausnummern produktiv umnutzten, um Zustellbarkeit zu gewährleisten und damit - medienhistorisch gelesen - Kommunikation unter Abwesenden unabhängig von einem bestehenden »Lokalgedächtnis ${ }^{4}$ realisierten. Damit wurde der Grundstein für das Prinzip der Adressierbarkeit gelegt, wie es heute omnipräsent ist. Denn mit dem anschriftlichem Signum Adresse wird in der aktuellen Medienkulturlandschaft noch immer das Ziel einer herzustellenden Verbindung bezeichnet, gleich ob der Begriff die Postanschrift oder eine E-Mail-Adresse, eine IPAdresse, einen Datenbankschlüssel oder eine Speicheradresse bezeichnet. Damit benennt das Konzept der Adresse einen medienwissenschaftlichen Grund-

2 Die Begriffe `menschlicher beziehungsweise `nicht-menschlicher Akteur gehen auf die Akteur-Netzwerk-Theorie (ANT) zurück, die Netzwerke auf den kritischen Anteil menschlicher und nicht-menschlicher Akteure an und in ihnen nach symmetrischem Prinzip hin befragt. Siehe bspw. Jim Johnson [Bruno Latour]: Mixing Humans and Nonhumans Together. The Sociology of a Door-Closer, in: Social Problems 35.3 (1988), S. 198-310 oder Bruno Latour: Science in Action. How to Follow Scientists and Engineers through Society, Cambridge 1987.

3 Elena Fingerhut: Übertragen und Speichern. Zum Verhältnis von Adressen und medialen Gehäusen, in: Christina Bartz, Timo Kaerlein, Monique Miggelbrink u.a. (Hrsg.): Gehäuse. Mediale Einkapselungen, Paderborn 2019, S. 343-361.

4 Markus Krajewski: Zettelwirtschaft. Die Geburt der Kartei aus dem Geiste der Bibliothek, Berlin 2002, S. 36. 
begriff, der sich postalischen Praktiken des Zustellens auf Basis von identitätsstiftender Nummerierung verdankt. Diese Fiktion persönlicher Anschriften bestimmt noch immer maßgeblich unsere E-Mail-Kommunikation, denn »[w]ie die Post braucht auch ein Netzwerk den Namen und die Adresse des Empfängers, um den richtigen Zielort für die Pakete zu kennen. ${ }^{5}$

Dass Fragestellungen der Postgeschichte und der Medienwissenschaft - neben den beiden angeführten Beispielen - notwendigerweise auf grundlegende Weise systematisch miteinander verwoben, verschränkt und überlagert sind, mag selbstredend nicht erstaunen. ${ }^{6}$ Schließlich eint beide Disziplinen der zentrale Begriff der Kommunikation und die Frage nach der Dimension seiner medialen, institutionellen und infrastrukturellen Bedingtheit, verbunden mit einem Fokus auf die maßgeblichen Techniken, die es Kulturen erlauben, für sie relevante Daten und Dinge zu speichern und zu übertragen (medienwissenschaftlich, allerdings nicht posthistorisch von Belang wäre zudem das Prozessieren $)^{7}$.

Auch dieser Beitrag wird die Verwobenheit von Post- und Mediengeschichte auf Basis eines konkreten Artefakts des französischen Ingenieurs Charles Bontemps aus dem Kontext der Post, nämlich der Rohrpost, thematisieren. Dabei geht es weniger um Situationen des Funktionierens von Rohrposten, sondern um das Wieder-Herstellen von Ordnung nach Störungen. Das besondere Potenzial von Störungen für die theoretische Reflexion von technischen Medien sowie von Medien- und Kommunikationssystemen ist bereits betont worden, da sich Störungen nicht allein als destruktiv erweisen müssen, sondern umgekehrt, produktiv werden können, da sie »konstitutiv für die Entstehung neuer Ordnung « sind. ${ }^{8}$ Im Sinne der im kanonischen Beitrag »A Mathematical Theory of Communication « von Claude Shannon präsentierten Schematisierung einer allgemeinen Kommunikationsanordnung, die den störenden

5 Michael Dertouzos: What Will Be? Die Zukunft des Informationszeitalters, Wien/New York 1999, S. 481. Die Metaphern des Postalischen nehmen im digitalen Raum nicht ab, sondern vielmehr zu. Ein Beispiel wäre das so genannte Post Office Protocol (POP), das dem Abrufen der allseits bekannten electronic mails von Servern dient.

6 Darauf hat bereits Harold Innis in seinem für die Mediengeschichte programmatischen Grundlagenwerk Empire and Communications von 1950 hingewiesen, in welchem er den Medientechniken des postalischen Transports und der Kommunikation entscheidende Bedeutung für die Prägung von Kulturen und den Aufstieg oder Fall von Weltreichen zugesteht. Siehe Harold Adams Innis: Empire and Communications, Oxford 1950.

7 Hierzu grundlegend Hartmut Winkler: Prozessieren. Die dritte, vernachlässigte Medienfunktion, Paderborn 2015.

8 Albert Kümmel, Erhard Schüttpelz: Medientheorie der Störung/Störungstheorie der Medien. Eine Fibel, in: dies. (Hrsg.): Signale der Störung, München 2003, S. 9-13, hier 9. 
Einfluss von externem »noise « auf Signalsendungen grafisch darstellt ${ }^{9}$ - diese ist zudem die Mutter aller Sender-Empfänger-Modelle -, könnte gar behaupten werden, dass erfolgreiche Kommunikation die fortwährende Arbeit an der Störung verlangt, was die Frage nach Störungen umso dringlicher werden lässt.

Bevor jedoch das erwähnte Artefakt von Bontemps historisch entfaltet wird, wird der Beitrag im Folgenden den problemrelevanten Kontext der Rohrpost im 19. Jahrhundert erläutern. Abschließend wird dargelegt werden, welche Bedeutung dem Artefakt medien- und technikhistorisch innewohnt und warum dieses zudem insbesondere für eine Wissens- und Technikgeschichte der Akustik von Interesse ist.

\section{Gestörte Rohrposten}

»Un train vient à s'arrèter [sic] dans le tube, que fait-on en pareil cas? ${ }^{10}$ Mit dieser Frage - Ein Zug kommt in der Röhre zum Stehen, was machen wir in so einem Fall? - eröffnete Charles Bontemps, dessen Name untrennbar mit dem Aufbau des Pariser Rohrpostsystems verbunden ist, das Kapitel über Störungen (Dérangements) seines Buches Les Systèmes Télégraphiques. Dieses Buch fasste alles $\mathrm{zu}$ seinerzeit Wissenswerte über optische beziehungsweise LuftTelegrafie (Télégraphiques Aériens), elektrische Telegrafie (Télégraphiques Électrique) und schließlich pneumatische Telegrafie oder schlechthin: Rohrpost (Télégraphiques Pneumatiques) zusammen. Anders formuliert war die erwähnte Frage die Frage nach der Lösungsstrategie gegen materielle Störungen im Rohrpostrohr respektive -kanal, die den Rohrpostbüchsen- und das heißt Nachrichten-Austausch verhinderten. Diese Frage, deren Beantwortung prima facie leicht fallen sollte, rüttelte im Veröffentlichungsjahr des Buchs, 1876, an einem Grundproblem der Rohrpost in der zweiten Hälfte des 19. Jahrhunderts. Denn mit der Einführung der Rohrpost wurde Kanalarbeit buchstäblich und die Frage nach dem Funktionieren von Kommunikation zu einer Frage nach etwaigen Störungen im Kanal. Oder mit Bontemps selbst kommentiert, der - wie bereits oben angekündigt - erfolgreiches Kommunizieren als fortwährende Arbeit an der Störung beschreibt: »Intimately connected with the working of the [pneumatic, C.B.] tubes is the removal of obstructions which occur from time to time, causing not unfrequently serious incon-

9 Claude Elwood Shannon: A Mathematical Theory of Communication, in: Bell System Technical Journal 27.3 (1948), S. 379-423.

10 Charles Bontemps: Les Systèmes Télégraphiques. Aériens - Électriques - Pneumatiques. Paris 1876 , S. 327. 
venience and delay. ${ }^{11}$ Und dieses »delay« galt dem System Rohrpost, wie jedem anderen Kommunikationssystem auch, als inakzeptables Übel. Schließlich war das System der Rohrpost eines, das materielle Kommunikation automatisierte und Postsendungen mit damals möglicher Höchstgeschwindigkeit transportierte. Schon die englische Bezeichnung Pneumatic Despatch für Rohrpost (heute eher Dispatch) ist dafür programmatisch. Nach dem Oxford English Dictionary bedeutet das Wort nämlich als Substantiv das Versenden von etwas (oder jemandem) zu einem Ziel (seien es Briefe, Truppen oder Rechnungen), ebenso als Verb aber auch das schnelle und effiziente Handhaben einer Aufgabe.

Störungen im Kanal der Rohrpost, namentlich steckengebliebene Rohrpostbüchsen, standen dem destruktiv entgegen. Denn Rohrposten erinnern daran, dass sämtliche Kommunikationsnetzwerke - wie ortslos das digitale world wide web auch erscheinen mag - immer räumlich rückgebunden und das heißt auf Kanäle, Röhren oder Kabel angewiesen sind, die störungsfrei zu funktionieren haben, um die Möglichkeit des Nachrichtenaustauschs zu garantieren. Rohrposten sind dafür ein geradezu exemplarisches Beispiel. Denn jede im Postrohr steckengebliebene Rohrpostbüchse machte sogleich den ganzen Kanal unbrauchbar. Schließlich waren die einzelnen Rohre der Rohrpost zwischen zwei Stationen materiell geschlossene Systeme, was bedeutete, dass der Luftdruck eines Rohrs nicht an beliebiger Stelle verändert werden konnte, sondern entweder Ergebnis von kontinuierlichem Überdruck der Sendestation oder kontinuierlichem Unterdruck seitens der Empfangsstation war. Technisch gesprochen war damit die Bandbreite des Kanals sehr limitiert. Steckten Botschaften einmal im Rohrpostrohr fest, konnten keine weiteren Nachrichten mehr durch das Rohr gesendet werden, bevor die Störung beseitigt war. War die Bedeutung der Störungsbeseitigung in technischen Kanälen seinerzeit in anderen Diskursen bereits brisant geworden - allen voran im Kontext von Telegraphen-Kabeln, besonders den meist vergleichsweise langen unterseeischen Kabeln ${ }^{12}$ - geriet damit nun die Störung als zentrales Element von Kommunikation in den Fokus von Ingenieuren, die sich mit materieller statt elektrotechnischer Übertragung beschäftigten.

Zur Störung eines Kanals, also dem Steckenbleiben einer Büchse im Rohrpostrohr, konnte es kommen, wenn 1.) der benötigte Luftdruck nicht ausreichte, um die Sendung zu befördern oder 2.) der Unterdruck an der Empfangssta-

11 Charles Bontemps: The Pneumatic Telegraphs of Paris, in: Minutes of Proceedings of the Institution of Civil Engineers 43 (1875), S. 116-134, hier S. 132.

12 Exemplarisch bspw. Latimer Clark: Testing for Faults, in: ders.: An Elementary Treatise on Electrical Measurement for the Use of Telegraph Inspectors and Operators, London 1868, S. 69-77. 
tion nicht ausreichte, wenn sich 3.) Wasser in den Rohren sammelte oder 4.) wenn sich die Röhren - vornehmlich im Winter - durch massiven Bodenfrost verengten. So wurde zwar die am 1. März 1893 in Betrieb genommene Rohrpostlinie in Philadelphia $1,2 \mathrm{~m}$ tief im Boden verlegt und war damit unterhalb der Frostgrenze, ${ }^{13}$ dahingegen war aber ein Großteil der Berliner Rohrpost (geplant und gebaut von Siemens \& Halske, deren erste Strecke 1865 zwischen dem zentralen Telegraphengebäude und der Börse eröffnet wurde) lediglich zwei bis drei Fuß "unter dem Straßenpflaster" verlegt worden, wie Werner Siemens im selben Jahr, 1865, berichtete. ${ }^{14}$ Dadurch war die Berliner Rohrpostanlage in ihren Anfangsjahren wesentlich wetterabhängig, wie es auch 1867 kommentiert wurde: Durch die geringe Tiefe der Rohrpost-Rohre unter den Gehwegen

»kam es, besonders bei feuchtem Wetter vor, daß das in der verdichteten, erwärmten Luft enthaltene Wasser in den kalten Röhren condensirte und dadurch die Telegrammbüchsen nebst Inhalt in den Röhren feucht wurden, im Winter aber, sobald das Wasser gefror, in denselben stecken blieben. ${ }^{15}$

\section{Uneinsichtige Rohre}

Aufgrund dieses geschilderten Problems geriet der Kanal als die intermediäre Instanz der Rohrpost-Kommunikation, die allenfalls passive Erwähnung in den Schriften zur Rohrpost findet, nämlich als etwas, das es innerhalb möglichst kurzer Zeiten zu überwinden galt, ${ }^{16}$ in den Fokus der Aufmerksamkeit. In den zu ihrer Zeit gängigen Verfahren der Störungsbeseitigung bei Steckenbleiben einer Rohrpostsendung im Kanal zählte es, durch eine Umkehrung des Luftdrucks die Büchse wieder zu ihrem Ausgangspunkt zurück zu befördern. Eine zweite Möglichkeit war es, auf der bereits gefahrenen Strecke der Postbüchse einen Unterdruck zu erzeugen, was dasselbe bewirken sollte.

13 Ingmar Arnold: Luft-Züge. Die Geschichte der Rohrpost, Berlin 2016, S. 106.

14 Werner Siemens: Die pneumatische Depeschenbeförderung zwischen der Central-Telegraphenstation in Berlin und dem Bösengebäude daselbst, in: ders.: Wissenschaftliche und Technische Arbeiten. Zweiter Band. Technische Arbeiten, Berlin 1891 [1865], S. 217-229, hier S. 227.

15 Zit. n. Arnold, Luft-Züge, S. 120. Rechtschreibung folgt dem Original.

16 Bspw. heißt es über die Berliner Rohrpost 1909: „Die längste Fahrt zwischen zwei einzelnen Ämtern geht über 3 Kilometer; offiziell braucht man zum Überwinden dieser Distanz vier Minuten, 19 Sekunden bei komprimierter Luft sowie fünf Minuten dreißig Sekunden bei verdünnter Luft. Die höchsten Geschwindigkeiten werden bei kürzeren Fahrten erzielt, wo eine Höchstgeschwindigkeit von 20 Metern/Sekunde bei komprimierter Luft und 16 Metern/Sekunde mit verdünnter Luft erreicht werden. "Anonym: Investigations as to Pneumatic Tube Service for Mails, Washington 1909, hier zitiert nach Arnold, Luft-Züge, S. 133. 
Ließ sich die Störung mit all diesen Prozeduren nicht beseitigen - anders gewendet: präsentierte sich das Rohrpostrohr statt als passiver Überträger von Nachrichten als unbeabsichtigter Speicher eben jener Nachrichten -, »so bleibt freilich nichts anders übrig «, so Werner Siemens 1865, »als den Röhrenstrang aufzunehmen«, um die steckengebliebene Büchse manuell zu entfernen. Das zentrale Problem aber, Siemens weiter, war, dass sich die Störung »irgendwo in der Leitung « befinden konnte, wobei die Betonung auf diesem Irgendwo der buchstäblich uneinsichtigen Röhre lag. Um die Röhre zu öffnen, damit die Störung manuell beseitigt werden konnte, musste also, so Siemens, »der Ort, wo der Wagen sitzt, wenigstens annähernd bekannt sein « ${ }^{17}$, schließlich war es wenig ökonomisch, das ganze Rohr zu öffnen. So wurde zum historisch ersten Mal die Frage nach dem Wo eines Dings, das man weder sehen, noch hören, noch riechen kann, zum kritischen Moment von Kommunikationstechnik. Wie also lässt sich der Ort von etwas wissen, auf das kein direkter Zugriff möglich ist? Anders gefragt, wie misst man die Entfernung zu einem Ding, von dem keiner weiß, wo es ist?

Wichtig vorab für die Beantwortung dieser Fragen sowie für das Verständnis der Brisanz von Störungen im Kontext der Rohrpost und der erheblichen Schwierigkeiten, die diese mit sich brachten, ist zunächst ein Blick auf ihren historischen Kontext. Die in der zweiten Hälfte des 19. Jahrhunderts entstehenden Rohrpostnetze in Paris, Marseille, Berlin, München, Hamburg, Wien, New York und Boston - um nur einige der größten zu nennen - waren Infrastrukturen, die die Großstädte netzartig durchzogen (so genannte Fernrohrpostanlagen im Gegensatz zu Innenrohrpostanlagen). Vornehmlich in Bezug auf konkrete Fallbeispiele sind diese frühen Rohrpostsysteme historisch bereits erschlossen worden. Für eine historische Aufarbeitung beispielsweise der Wiener Rohrpost sei auf Walther Turner ${ }^{18}$ oder Hans Hajek ${ }^{19}$ verwiesen, mit Bezug auf Berlin legte Ingmar Arnold eine umfassende Arbeit vor. ${ }^{20}$ Auch sind an

17 Siemens, Die pneumatische Depeschenbeförderung, S. 217-229, hier S. 227. Mit »Wagen« ist dabei die Rohrpostbüchse gemeint. Diese Äquivalenz von Nachrichten- und Transporttechnik in der Bezeichnung gilt auch insofern mehrere "Wagen« zu so genannten "Zügen« gruppiert wurden. Im Englischen erfolgte die Bezeichnung der Rohrpostbüchsen und ihre Aneinanderreihung mit »wagon" und »train« demselben rhetorischen Schema.

18 Walther Turner: Die Stadtrohrpost in Wien 1875-1956, Wien 1978.

19 Hans Hajek: Geschichte der Wiener Rohrpost, Wien 1933. Zudem von Interesse für die Wiener Rohrpost: Christine Kainz: Österreichs Post. Vom Botenposten zum Postboten, Wien 1995, insb. S. 113-117; allgemein auch: Florian Bettel: »Der >vollkommenen Welt um einen Schritt näher.» Die Rohrpost am Arbeitsplatz in fünf Bildern, in: Blätter für Technikgeschichte 73 (2011), S. 127-148.

20 Ingmar Arnold: Luft-Züge. Die Geschichte der Rohrpost in Berlin und anderswo, Berlin 2000. Zur Berliner Rohrpost siehe auch: Reinhard Krüger: Pneumatische Streifzüge I zur Geschichte der Berliner Rohrpost (1863-1976), Frankfurt am Main 2013, oder Wolfgang Wengel: Come- 
dieser Stelle die umfassenden Arbeiten von Reinhard Krüger zur Rohrpost erwähnenswert. $^{21}$

Diese und andere historische Darstellungen innerstädtischer Rohrpostanlagen illustrieren, dass - im Gegensatz zu den noch heute gebräuchlichen Rohrpostsystemen, die die Grenzen eines Gebäudes meist nicht übersteigen ${ }^{22}$-, die Rohre zwischen den einzelnen, miteinander verbundenen innerstädtischen Sende- und Empfangsstationen mitunter recht lang waren. Sie waren nicht im Bereich weniger, sondern hunderter Meter, teilweise sogar im Kilometerbereich. Beispielsweise erstreckte sich das Berliner Rohrpostnetz bereits 1868 über eine Länge von 18 Kilometern. ${ }^{23}$ Als am 1. Dezember 1876 die Berliner Rohrpost auch für die Öffentlichkeit zugänglich gemacht wurde, verfügte sie bereits über 15 Rohrpostämter mit einer gesamten Ausdehnung von rund 26 Kilometern Rohrlänge. Dass die innerstädtischen Rohrpostnetze tendenziell das Zentrumsgebiet der jeweiligen Städte vollständig abzudecken versuchten, zeigen ihre schematischen Darstellungen, von denen hier repräsentativ zwei zur Illustration herhalten sollen: Ein Plan des Rohrpostnetzes von Paris aus dem Jahr 1888 (siehe Abbildung 1) und die Schematisierung des Rohrpostnetzes von Berlin aus dem Jahr 1884 (siehe Abbildung 2).

\section{Arbeit an der Störung}

Aufgrund der Ausmaße dieser Fernrohrpostanlagen war das Auffinden steckengebliebener Rohrpostbüchsen mit konventionellen Verfahren nicht mehr möglich. Denn die bereits bekannte Methode, eine steckengebliebene Postbüchse mit Hilfe von Draht beziehungsweise einer Gelenkstange zu lokalisieren, die von Seiten einer Sende- oder Empfangsstation in das gestörte Rohr geschoben wurde, erübrigte sich, da dies nur auf eine Entfernung von etwa $60 \mathrm{~m}$ glückte. Vonnöten wurde die Konzeption und Realisierung neuer Strategien zur Störungslokalisation, damit die gestörten Rohre möglichst nah an der

back der Rohrpost? 125 Jahre Stadtrohrpost Berlin - auch heute noch ein Vorbild für technische Innovation, in: Das Archiv 1.2 (2002), S. 6-19.

21 Reinhard Krüger: Die Rohrpost von Marseille, Berlin 2017; ders.: Studien und Quellen zur Geschichte der Stadtrohrpost Hamburg bis 1935, Berlin 2015; ders.: Die Rohrpost von Algier, Berlin 2015.

22 So verfügt noch heute (2019) beispielsweise das Bundeskanzleramt in Berlin über ein internes Rohrpostsystem, sowie auch das Heidelberger Universitätsklinikum. Zweiteres verdeutlicht, dass sich die innerbetriebliche Rohrpost heutzutage vor allem noch dort bewähren kann, wo es gilt, nicht ausschließlich Daten, sondern auch Dinge respektive Flüssigkeiten (Medikamente, Blut) möglichst schnell und personal-ökonomisch zu versenden.

23 Die größte Ausdehnung erreichte das Berliner Rohrpostnetz im Jahr 1940 mit einer gesamten Rohrlänge von insgesamt knapp 400 Kilometern. 
Störung geöffnet werden konnten, um die steckengebliebene Rohrpostbüchsen manuell zu entfernen.

Von einem hierzu genutzten Verfahren berichtet der bereits zur Rohrpost zitierte Werner Siemens. Seine Lösung des Problems der Störungslokalisierung im Kanal operierte auf Basis von Rohrdurchmesser multipliziert mit der Länge des Rohrs bis zur Störung. In anderen Worten, es basierte auf dem Volumen des Rohrs bis zur steckengebliebenen Rohrpostbüchse. Um dieses zu ermitteln, verwendete er ein technisches Objekt, das sein Bruder Carl Wilhelm Siemens einige Jahrzehnte zuvor massiv verbessert und für welches er 1852 ein englisches Patent erhalten hatte: Den Wassermesser, der Wasserverbrauch direkt anzeigte und damit vom Prinzip her ein Wassermesser ist, wie er noch heute verwendet wird. ${ }^{24}$ Unter Einschaltung dieses Wassermessers galt es Werner Siemens, Wasser in einer Ledermanschette in das gestörte Rohr zu leiten, bis dieses zwischen Poststation und Störung vollständig mit Wasser gefüllt war. ${ }^{25}$ Dieses durchaus primitive, dennoch wirksame Verfahren war allerdings mit dem Problem verbunden, dass zwar gemäß Wasservolumen die Position der festsitzenden Rohrpostbüchse - bei bekanntem Rohrdurchmesser - annähernd exakt bestimmt werden konnte, das Rohr aber anschließend wieder leergepumpt werden musste, was in der Praxis der Rohrpost zu Problemen führte, da dies sehr zeitaufwendig war.

Ein zweites in der Rohrpost-Praxis angewandtes Verfahren, die unbekannte Position steckengebliebener Rohrpostbüchsen zu bestimmen, d.h. Störungen $\mathrm{zu}$ lokalisieren, das ebenso indirekt funktionierte wie das bereits geschilderte, basierte auf Luftdruck-Messungen, beziehungsweise genauer: Der Differenz zweier Luftdruck-Messungen. Dabei wurde die Veränderung des Luftdrucks eines Drucklufttanks in der Rohrpoststation des gestörten Kanals auf einem Manometer (ein Druckmessgerät) beobachtet, wenn dieses nacheinander mit einer Linie bekannter Länge und anschließend mit der gestörten Rohrpostlinie in Verbindung gebracht wurde. Die Druckdifferenzen konnten dann auf dem Manometer abgelesen werden. Dieses war zwar nicht auf Entfernungen geeicht, bei ausreichender Empirie des Postmitarbeiters, also bei genügend praktischer Erfahrung, konnte dieser an der Skalierung des Manometers jedoch bei bekannter Länge des Referenzrohres die Störungsstelle annähernd bestimmen. Andererseits - und ebenso basierend auf Luftdruck - konnte bei bekanntem Anfangs- und Enddruck im betreffenden, gestörten Rohr die Fehlerstelle bestimmt werden, da sich ihre Position proportional zum Luftdruck zwischen Anfangs- und Enddruck im Rohr verhält. Hierzu war wiederum ein Manome-

24 Für einen historischen Abriss über heimische Wasserzähler siehe L. Sell: Die Wassermesser für Hausleitungen, in: Polytechnisches Journal 301 (1896), S. 241-248.

25 Siemens, Die pneumatische Depeschenbeförderung, S. 227. 
ter von Nöten. Gemäß des physikalischen Gesetzes von Boyle-Mariotte waren Störungsstellen damit mathematisch bestimmbar. Erschwert wurden diese Prozeduren allerdings durch das Ablesen des Barometers, das millimetergenau $\mathrm{zu}$ erfolgen hatte, was in der Praxis meist schwer umzusetzen war. Allerdings konnte auch so die Position der steckengebliebenen Rohrpostbüchse nur bis auf höchsten $30 \mathrm{~m}$ genau bestimmt werden. ${ }^{26}$ Damit war das Verfahren $\mathrm{zu}$ ungenau.

Der bereits eingangs erwähnte französische Ingenieur und Pariser Rohrpost-Pionier Charles Bontemps (1839-1884) berichtete demgegenüber über eine seinerzeit neue, aus heutiger Perspektive genuin moderne Methode, die Entfernung zu einer steckengebliebenen Rohrpostbüchse zu ermitteln. Eine Methode, die - so scheint es in der Retrospektive - ihrer Zeit weit voraus war, insofern apparative Verfahren, die auf gleichen Epistemen fußen, erst Jahrzehnte später breite Wirkmächtigkeit entfalten sollten. Sein Verfahren nebst der verwendeten Apparatur als neuem »epistemischen Ding ${ }^{27}$ in der postalischen Praxis markiert den historischen Ursprung einer ganzen Reihe unterschiedlicher Techniken der Entfernungsbestimmung. Um diese Apparatur, ihre Herkunft und ihren originären Zweck zu verstehen, ist jedoch ein kleiner historischer Umweg erforderlich, der beim französischen Chemiker und Physiker Henri Victor Regnault (1810-1878) beginnt.

Regnault beschäftigte sich in den 1860er Jahren mit der Messung von Schallgeschwindigkeit, die bis dato noch keineswegs als gesicherte Größe galt. Dies machte er exakt in der Stadt, in welcher Bontemps später maßgeblich an der Installation des dortigen Rohrpostsystems beteiligt sein sollte - Paris -, zudem an genau jenem Ort, in welchem die spätere Rohrpost verlegt werden sollte - der Pariser Kanalisation - und nicht zuletzt war es Regnaults erklärtes Ziel, die Schallgeschwindigkeit in den Objekten zu bestimmen, welche auch für die Rohrpost konstitutiv werden sollten: In Röhren.

Regnaults apparatives Verfahren basierte auf dem Öffnen und anschließendem Schließen eines zunächst geschlossenen Stromkreises, wobei jenes Öffnen und Schließen am Anfang beziehungsweise Ende einer Röhre bekannter Länge geschah. Regnault feuerte eine ungeladene Pistole am Anfang jener Röhre ab, wodurch ein akustischer Impuls erzeugt wurde, dessen Druckwelle einen feinen Draht durchtrennte (und damit den Stromkreis öffnete) und der sich anschließend durch die Röhre fortpflanzte. Am Ende der Röhre bewirkte die akustische Welle eine Wölbung eines zweiten feinen Drahts, wodurch der

26 Bontemps, Les Systèmes Télégraphiques, S. 328.

27 Zum Begriff des epistemischen Dings siehe insbesondere Hans-Jörg Rheinberger: Experimentalsysteme und epistemische Dinge. Eine Geschichte der Proteinsynthese im Reagenzglas, Göttingen 2001. 
Stromkreis wieder geschlossenen wurde. Kritisches Moment der Messanordnung war es nun, die Zeit zwischen Öffnen und Schließen des Stromkreises möglichst exakt zu bestimmen, um die bekannte Rohrlänge durch dieses Zeitintervall dividieren zu können, um sodann einen Wert für die Schallgeschwindigkeit mathematisch bestimmbar zu machen. ${ }^{28} \mathrm{Zu}$ diesem Zweck war das Öffnen und Schließen des Stromkreises mit einem Elektromagneten synchronisiert, der entsprechend seines Zustands (magnetisiert/nicht-magnetisiert) einen Hebel anzog oder nicht. Wurden diese beiden Zustände des Hebels auf einer sich drehenden Walze mit berußtem Papier und zudem ein Maßstab für die Zeit verzeichnet, konnte das zu bestimmende Zeitintervall buchstäblich gesehen und ausgezählt werden, da ein zeitliches Ereignis in ein räumliches nämlich ein graphisches - übersetzt worden war. Fünf Jahre lang, zwischen 1862 und 1866, bestimmte Regnault die Schallgeschwindigkeit in Gas- und (Ab-)Wasserleitungsröhren in Paris mit diesem Verfahren. Er nutzte dazu Rohre mit einer Länge von bis zu $20 \mathrm{~km}$ und einem Durchmesser zwischen rund $10 \mathrm{~cm}$ und $1 \mathrm{~m}$ und konnte damit recht konkrete Werte für die Schallgeschwindigkeit in Röhren unterschiedlichen Durchmessers bei unterschiedlichen Temperaturen bestimmen.

\section{Die Räumlichkeit des Schalls}

Regnault brachte also die Räumlichkeit des Schalls in Röhren unterschiedlichen Durchmessers zum ersten Mal auf konkrete Werte und er entwarf und beschrieb eine Messanordnung, die es potenziell jedem Interessierten erlaubte, seine Versuche zu reproduzieren. Das Novum und die Pionierleistung Bontemps' war es, diese Regnault'sche Apparatur zu verwenden, aber mit dieser eine andere Frage zu beantworten, insofern er die bekannten und unbekannten Parameter der Messanordnung produktiv miteinander vertauschte. Bontemps galt es nicht mehr, bei bekannter Länge eines Rohres eine vormals unbekannte Schallgeschwindigkeit $\mathrm{zu}$ ermitteln, sondern bei nunmehr hinreichend bekannter Schallgeschwindigkeit den unbekannten Parameter Rohrlänge - nämlich den Abstand zwischen einer Rohrpoststation und einer steckengebliebenen Rohrpostbüchse - akustisch zu messen. Bontemps adaptierte also Regnaults Verfahren unter vorzeichenverkehrten Bedingungen. Über die Neu-

28 Dieses Verfahren der Bestimmung von Zeitintervallen auf Basis des Öffnens und Schließens eines Stromkreises war im historischen Kontext als »zeitmessender Strom« bekannt und entstammt der experimentalpraktischen Ballistik und Physiologie, genauer: Der Elektrophysiologie. Prominenten Einsatz erfuhr das Verfahren zur Mitte des 19. Jahrhunderts in Hermann von Helmholtz' Messung der Nervenleitgeschwindigkeit. 
artigkeit dieser Messtechnik (L'originalité de ces expériences) im Dienste aktiver akustischer Ortung war sich Bontemps schon seinerzeit durchaus bewusst. ${ }^{29}$

Zudem überführte Bontemps die bis dato vollkommen zweckfreie Forschung Regnaults in den Bereich des Praktischen, indem er die Konkretisierung der Geschwindigkeit des Schalls zum Zwecke der Vermessung des Raums (nämlich von Röhren) nutzte. Damit realisiert die Apparatur nebst ihrer praktischen Implementierung das historisch erste technische Verfahren des remote sensing auf Basis von Laufzeitmessung und - das ist insbesondere medienhistorisch von Bedeutung - ist somit das erste apparative Verfahren der Echoortung. ${ }^{30}$ Denn der einzige Unterschied in Bontemps' Apparatur im Gegensatz zu der von Regnault war, dass nicht mehr am Ende eines Rohres der Zeitpunkt zur Markierung kam, wenn der Schall des Pistolenschusses dieses erreichte, sondern jeder Zeitpunkt markiert wurde, wenn das Echo von einer steckengebliebenen Rohrpostbüchse wieder am Anfang des Rohres eintraf.

Die mechanische Komplexität des Verfahrens wird in einer Abbildung deutlich, die Bontemps in seinem Buch über Telegrafie zeigte (siehe Abbildung 3). Von besonderem Interesse ist dabei die zentral zu sehende Zylinderwalze mit berußtem Papier, auf der insgesamt drei Schwingungsvorgänge chronographisch verzeichnet werden (und damit das so genannte Chronogramm erzeugen), um das Zeitintervall zwischen Ortungsimpuls (Pistolenschuss) und seinem ebendort wieder empfangenen, an einer steckengebliebenen Rohrpostbüchse reflektierten Echo möglichst exakt zu bestimmen. Damit diese Translation einer Zeit- in eine Raumdifferenz auf dem berußten Papier tatsächlich auch mathematisierbar, d.h. in numerische Werte überführbar war, wurden neben den sukzessiven Echos weiterhin die Schwingung eines Sekundenpendels binär verzeichnet als auch die Schwingungen einer Stimmgabel, die - in diesem Fall - eine Frequenz von $33 \mathrm{~Hz}$ hatte, d.h. 33 Schwingungen pro Sekunde, was aufgrund der Verzeichnung des Sekundenpendels ersichtlich war.

Damit war das Verfahren der Ortung von Störungen im Rohrpostkanal praktisch eine chronographische Operation, nämlich das Abzählen von Stimmgabelschwingungen, die zwischen einem Echo und dem nächsten vergingen, wie es Bontemps auch selbst berichtete: "Si l'on réussit à évaluer l'intervalle de temps écoulé entre les apparitions des deux marques, il est aisé de voir qu'on pourra calculer la distance de la membrane à l'obstacle.« (»Wenn es uns gelingt, das Zeitintervall zwischen zwei Markierungen [d.h. zwei sukzessiven Echos] zu bestimmen, ist es leicht zu sehen, dass wir die Entfernung zwischen Membran und Hindernis [die steckengebliebene Rohrpostbüchse]

29 Bontemps, Les Systèmes Télégraphiques, S. 329.

30 Remote sensing, zu deutsch Fernerkundung. 
berechnen können.«) $)^{31}$ Diese Anzahl an Stimmgabelschwingungen zwischen zwei Echos wurde sodann durch zwei dividiert, da es sich schließlich um ein Echo handelt und somit die doppelte Schallstrecke zurückgelegt wurde. Dieses Ergebnis wurde mit der von Regnault ermittelten Schallgeschwindigkeit (rund $330 \mathrm{~m} / \mathrm{s}$ ) multipliziert. Das heißt, ein Zeitereignis wurde zunächst in einen Graphen übersetzt, mithin visualisiert, um aus dieser Visualisierung wiederum das Zeitereignis mathematisch möglichst exakt zu bestimmen, um letztlich eine räumliche Distanz errechenbar zu machen. Damit stand am Ende dieser Operationskette eine Entfernungsangabe, die im Idealfall möglichst exakt mit der Entfernung zwischen Rohrpoststation und steckengebliebener Rohrpostbüchse korrespondierte. An jener Stelle wurde schließlich das gestörte Rohrpostrohr geöffnet und die Büchse manuell entfernt. Die mit jeder Ortung notwendig einhergehende chronographische Visualität ist dabei noch nicht als "Phänomenotechnik« im Sinne Gaston Bachelards zu bewerten. ${ }^{32}$ Allerdings wurde es mit dem Verfahren möglich, Aussagen über ein tatsächlich unsichtbares Objekt zu treffen, nämlich Aussagen über die Position einer Rohrpostbüchse. Denn die Rohre großer Rohrpostsysteme sind buchstäblich verschlossen; die Störungen in ihnen waren materiell, allerdings entzogen sie sich den menschlichen Sinnen. Aus diesem Grund stellt das Verfahren ein in höchstem Grade mediales dar: Es erzeugt technisch Einsichtigkeit ansonsten uneinsichtiger Rohre.

Aus den überlieferten Quellen lässt sich ersehen, dass diese Form der Echoortung im Rahmen der Rohrpost in der zweiten Hälfte des 19. Jahrhunderts tatsächlich praktiziert wurde. ${ }^{33}$ Wie viele Apparate, die Bontemps chronographe enregistreur nannte (zu deutsch etwa chronographisches Registriergerät), international Verwendung fanden, lässt sich allerdings nur schwerlich rekonstruieren. Dies schränkt das Novum der apparativen Methode aber weder ein, noch ändert es etwas daran, dass es das historisch erste technische Verfahren der Echoortung war; ein Verfahren, welches - dazu abschließend mehr - seit dem 20. Jahrhundert in unterschiedlichsten Kontexten der Echoortung und Laufzeitmessung erneut technisch brisant werden sollte. Dass das Verfahren

31 Bontemps, Les Systèmes Télégraphiques, S. 329.

32 Bachelard prägte den Begriff der »Phänomenotechnik« in den 1930er Jahren und referiert mit diesem unter anderem auf wissenschaftliche Bilder, die das, was sie zu zeigen vorgeben, selbst erst hervorbringen und damit selbst zu Erkenntnisobjekten werden. Zum Begriff siehe auch Hans-Jörg Rheinberger: Gaston Bachelard und der Begriff der `Phänomenotechnik», in: Marc Schalenberg, Peter T. Walther (Hrsg.): ‘.. immer im Forschen bleiben«. Rüdiger vom Bruch zum 60. Geburtstag, Stuttgart 2004. S. 297-310.

33 Max Jüllig: Ueber akustische Distanzmessung. Vortrag, gehalten am 17. November 1880, in: Schriften des Vereins zur Verbreitung naturwissenschaftlicher Kenntnisse 21 (1881), S. 55-87, hier S. 86. 
im 19. Jahrhundert allerdings noch ein Nischendasein fristete, lässt sich auch daran ablesen, dass es fortwährend singulär an den Namen Bontemps gekoppelt ist und sich die Zirkulation seiner Beschreibung entsprechend ausgestaltete. Bontemps berichtete über das Verfahren zunächst 1873 auf französisch im Journal de Physique Théorique et Appliquée, ${ }^{34} 1874$ wurde der kurze Artikel identisch in englischer Übersetzung im Journal of the Society of Telegraph Engineers abgedruckt ${ }^{35}$ und 1875 erneut in den Minutes of Proceedings of the Institution of Civil Engineers. ${ }^{36} 1876$ beschrieb Bontemps das Verfahren erneut in seiner bereits erwähnten Übersichtsdarstellung telegraphischer Verfahren, ${ }^{37}$ 1881 wurde die Beschreibung erneut identisch im Dictionary of Engineering abgedruckt. ${ }^{38}$ Noch 1897 druckte Birney Clark Batcheller, der die erste USamerikanische Rohrpost in Philadelphia und später die New Yorker Rohrpost konzipierte, die Beschreibung abermals in seiner Überblicksdarstellung über pneumatische Postanlagen ab. ${ }^{39}$ Batcheller fügte dem Nachdruck der Beschreibung Bontemps' den Kommentar hinzu, dass er selbst von der Beseitigung von Störungen in den Rohren »no experience « habe und deshalb Bontemps zitieren müsse - dessen Artikel zu diesem Zeitpunkt wohlgemerkt schon 24 Jahre alt war.

\section{Resümee}

Dass das Auffinden von Störungen in den Kanälen der Pariser Rohrpost ein Problem war, das akustisch-chronographisch gelöst wurde, ist aus dem historischen und situierten Kontext heraus zu erklären. Regnaults Messung der Schallgeschwindigkeit in Röhren von unterschiedlichem Durchmesser war einerseits erst wenige Jahre jung und seine apparative Methode war Physikern ihrer Zeit durch umfangreiche Publikationen bekannt. ${ }^{40}$ Andererseits führte

34 Charles Bontemps: Note sur un procédé pour la détermination du point d'arrêt d'un convoi de dépêches dans les tubes pneumatiques, in: Journal de Physique Théorique et Appliquée 2.1 (1873), S. 257-260.

35 Charles Bontemps: Note on a Method of Discovering the Point of Stoppage of a Carrier in Pneumatic Tubes, in: Journal of the Society of Telegraph Engineers 3.7 (1874), S. 500-502.

36 Charles Bontemps: The Pneumatic Telegraphs of Paris, in: Minutes of Proceedings of the Institution of Civil Engineers Vol. 43 (1875), S. 116-134, hier 132-134.

37 Bontemps, Les Systèmes Télégraphiques.

38 Ernest Spon (Hrsg.): Supplement to Spons' Dictionary of Engineering, Civil, Mechanical, Military, and Naval. Division III, London 1881, S. 943.

39 Birney Clark Batcheller: The Pneumatic Despatch Tube System of the Batcheller Pneumatic Tube Co. Also Facts and General Information Relating to Pneumatic Despatch Tubes, Philadelphia 1897, S. 143-147.

40 Das apparative Verfahren Regnaults ist hierbei ein programmatisches Beispiel für so genannte »Selbstschreibeverfahren« beziehungsweise »Selbstschreibeapparaturen«, deren chronographi- 
Regnault seine Experimente in einem Raum durch, für den Paris seinerzeit weltbekannt und einzigartig war: Der Kanalisation, also exakt in jenem unterirdischen Netzwerk, in welchem sich der Pariser Rohrpost-Ausbau, geleitet von Bontemps, später vollziehen sollte. Gleichwohl die oben gegebene nüchterne Beschreibung des Verfahrens seine Brisanz fast zu negieren scheint, ist Bontemps' chronographe enregistreur dabei als posthistorisches Artefakt hinsichtlich einer Technikgeschichte der Akustik und vor allem medienhistorisch von erheblicher Signifikanz. Bontemps' inverse Adaption des Regnault'schen Verfahrens markiert den Anbeginn einer Mediengeschichte, die sich im 20. Jahrhundert in so bedeutenden Medientechniken wie dem Echolot, dem Sonar, der Sonografie, dem Radar oder dem Navstar GPS fortschreibt. Denn alle diese Medien messen zwar räumliche Entfernung, aber eigentlich Zeit, nämlich Zeitdifferenzen: Die Laufzeit akustischer (beziehungsweise im Fall von Radar und GPS elektromagnetischer) Impulse. Aus diesen Zeitdifferenzen zwischen Senden und Wieder-Empfangen eines Impulses wird erst eine räumliche Größe auf Basis der Schall- beziehungsweise Lichtgeschwindigkeit mathematisch ermittelt. Die epistemische Pionierleistung Bontemps' ist es, diese Laufzeit akustischer Impulse nicht mehr als zweckfreie Nebenbedingung jeder akustischen Übertragung anzusehen, sondern sie strategisch zu nutzen und dadurch Akustik zum ersten Mal zu Zwecken der Entfernungsmessung operationalisiert zu haben.

Techniken der aktiven Ortung sind aus dem neuzeitlichen mediatisierten Alltag nicht wegzudenken. Auf ihrer Basis wird der Luftraum organisiert und es wird navigiert (Radar), findet die Vermessung der Tiefen des Ozeans und das Auffinden von U-Booten statt (Echolot und Sonar), werden körperimmanente Strukturen untersucht (Sonografie) und in der Navigations- und Wegfindungspraxis ist durch so genannte smart devices und smart phones auch die Nutzung von GPS im zivilen Sektor alltäglich und ubiquitär geworden. Umso mehr mag erstaunen, dass die Geschichte der aktiven Ortungstechnik bisher noch nicht geschrieben wurde und folglich auch ihr Ursprung einer wissenschaftlichen Aufarbeitung harrt - und Bontemps Ortungsverfahren dadurch bisher unbeachtet blieb und damit ein wissenschafts-, technik- und medienhistorisches Desiderat darstellt.

Zwar mag sich die technisch-apparative Bedingung der Zeitmessung bei Bontemps vom Radar oder digitalen Verfahren der Zeitdifferenzmessung massiv unterscheiden, insofern sie sich - wie im 19. Jahrhundert üblicherweise praktiziert - chronographisch ausgestaltete. Der signifikante Aspekt ist jedoch

schen Kurvenbildern im 19. Jahrhundert die höchste Objektivität und größtmögliche Evidenz bescheinigt wurde. Hierzu siehe auch Stefan Rieger: Schall und Rauch. Eine Mediengeschichte der Kurve, Frankfurt am Main 2009. 
vielmehr, dass Bontemps eine akustische Übertragungsanordnung als Selbstzweck konstituierte, insofern eine Impulssendung selbstreflexiv geschah: Sie geschah allein, um den temporalen Index eben jener Sendung zu bestimmen, nämlich ihre Dauer, um aus dieser die Entfernung zu einem Objekt zu ermitteln, das sich den menschlichen Sinnen sonst entzieht. Es ging ihm also nicht um das akustische Übertragen semantisch sinnvoller Nachrichten, sondern es galt ihm, die Dauer von Übertragungen messtechnisch zu bestimmen. Diese Verzeitlichung des Raums als "Signum der Moderne ${ }^{41}$ und wie sie in derart ubiquitären Medien wie der Sonografie oder dem GPS basale Charakteristik ist, hat - wie gezeigt wurde - ihren epistemischen und technischen Ursprung in der Rohrpost. Einmal mehr erweist sich Postgeschichte damit als relevant für eine Geschichte der Medienkultur.

\section{Literaturverzeichnis}

Anonym: Investigations as to Pneumatic Tube Service for Mails, Washington 1909.

Ingmar Arnold: Luft-Züge. Die Geschichte der Rohrpost, Berlin 2016.

Birney Clark Batcheller: The Pneumatic Despatch Tube System of the Batcheller Pneumatic Tube Co. Also Facts and General Information Relating to Pneumatic Despatch Tubes, Philadelphia 1897.

Florian Bettel: »Der >vollkommenen Welt um einen Schritt näher.« Die Rohrpost am Arbeitsplatz in fünf Bildern, in: Blätter für Technikgeschichte 73 (2011), S. 127-148.

Charles Bontemps: Note sur un procédé pour la détermination du point d'arrêt d'un convoi de dépêches dans les tubes pneumatiques, in: Journal de Physique Théorique et Appliquée 2.1 (1873), S. 257-260.

Charles Bontemps: Note on a Method of Discovering the Point of Stoppage of a Carrier in Pneumatic Tubes, in: Journal of the Society of Telegraph Engineers 3.7 (1874), S. 500502.

Charles Bontemps: The Pneumatic Telegraphs of Paris, in: Minutes of Proceedings of the Institution of Civil Engineers 43 (1875), S. 116-134.

Charles Bontemps: Les Systèmes Télégraphiques. Aériens - Électriques - Pneumatiques, Paris 1876.

Latimer Clark: Testing for Faults, in: ders.: An Elementary Treatise on Electrical Measurement for the Use of Telegraph Inspectors and Operators, London 1868, S. 69-77.

Michael Dertouzos: What Will Be? Die Zukunft des Informationszeitalters, Wien/New York 1999.

Elena Fingerhut: Übertragen und Speichern. Zum Verhältnis von Adressen und medialen Gehäusen, in: Christina Bartz, Timo Kaerlein, Monique Miggelbrink u.a. (Hrsg.): Gehäuse. Mediale Einkapselungen, Paderborn 2019, S. 343-361.

Hans Hajek: Geschichte der Wiener Rohrpost, Wien 1933.

41 Jens Schröter: A momentary flash. Kurze Anmerkung zu sehr kurzen Lichtblitzen, in: Axel Volmar (Hrsg): Zeitkritische Medien, Berlin 2009, S. 167-175, hier S. 171. 
Harold Adams Innis: Empire and Communications, Oxford 1950.

Jim Johnson [Bruno Latour]: Mixing Humans and Nonhumans Together. The Sociology of a Door-Closer, in: Social Problems 35.3 (1988), S. 198-310.

Max Jüllig: Ueber akustische Distanzmessung. Vortrag, gehalten am 17. November 1880, in: Schriften des Vereins zur Verbreitung naturwissenschaftlicher Kenntnisse 21 (1881), S. 55-87.

Christine Kainz: Österreichs Post. Vom Botenposten zum Postboten, Wien 1995.

Markus Krajewski: Zettelwirtschaft. Die Geburt der Kartei aus dem Geiste der Bibliothek, Berlin 2002.

Reinhard Krüger: Pneumatische Streifzüge I zur Geschichte der Berliner Rohrpost (18631976), Frankfurt am Main 2013.

Reinhard Krüger: Studien und Quellen zur Geschichte der Stadtrohrpost Hamburg bis 1935, Berlin 2015.

Reinhard Krüger: Die Rohrpost von Algier, Berlin 2015.

Reinhard Krüger: Die Rohrpost von Marseille, Berlin 2017.

Albert Kümmel, Erhard Schüttpelz: Medientheorie der Störung/Störungstheorie der Medien. Eine Fibel, in: dies. (Hrsg.): Signale der Störung, München 2003, S. 9-13.

Bruno Latour: Science in Action. How to Follow Scientists and Engineers through Society, Cambridge 1987.

Hans-Jörg Rheinberger: Experimentalsysteme und epistemische Dinge. Eine Geschichte der Proteinsynthese im Reagenzglas, Göttingen 2001.

Hans-Jörg Rheinberger: Gaston Bachelard und der Begriff der `Phänomenotechnik«, in: Marc Schalenberg, Peter T. Walther (Hrsg.): ‘.. immer im Forschen bleiben`. Rüdiger vom Bruch zum 60. Geburtstag, Stuttgart 2004, S. 297-310.

Stefan Rieger: Schall und Rauch. Eine Mediengeschichte der Kurve, Frankfurt am Main 2009. Jens Schröter: A momentary flash. Kurze Anmerkung zu sehr kurzen Lichtblitzen, in: Axel Volmar (Hrsg): Zeitkritische Medien, Berlin 2009, S. 167-175.

L. Sell: Die Wassermesser für Hausleitungen, in: Polytechnisches Journal 301 (1896), S. 241248.

Claude Elwood Shannon: A Mathematical Theory of Communication, in: Bell System Technical Journal 27.3 (1948), S. 379-423.

Bernhard Siegert: Relais. Geschicke der Literatur als Epoche der Post, Berlin 1993.

Werner Siemens: Die pneumatische Depeschenbeförderung zwischen der CentralTelegraphenstation in Berlin und dem Bösengebäude daselbst, in: ders.: Wissenschaftliche und Technische Arbeiten. Zweiter Band. Technische Arbeiten, Berlin 1891 [1865], S. 217-229.

Ernest Spon (Hrsg.): Supplement to Spons' Dictionary of Engineering, Civil, Mechanical, Military, and Naval. Division III, London 1881.

Walther Turner: Die Stadtrohrpost in Wien 1875-1956, Wien 1978.

Wolfgang Wengel: Comeback der Rohrpost? 125 Jahre Stadtrohrpost Berlin - auch heute noch ein Vorbild für technische Innovation, in: Das Archiv 1.2 (2002), S. 6-19.

Hartmut Winkler: Prozessieren. Die dritte, vernachlässigte Medienfunktion, Paderborn 2015. 
Open-Access-Publikation im Sinne der CC-Lizenz BY-NC-ND 4.0 (C) 2021, Vandenhoeck \& Ruprecht GmbH \& Co. KG, Göttingen ISBN Print: 9783847112280 - ISBN E-Lib: 9783737012287 


\section{Abbildungen}

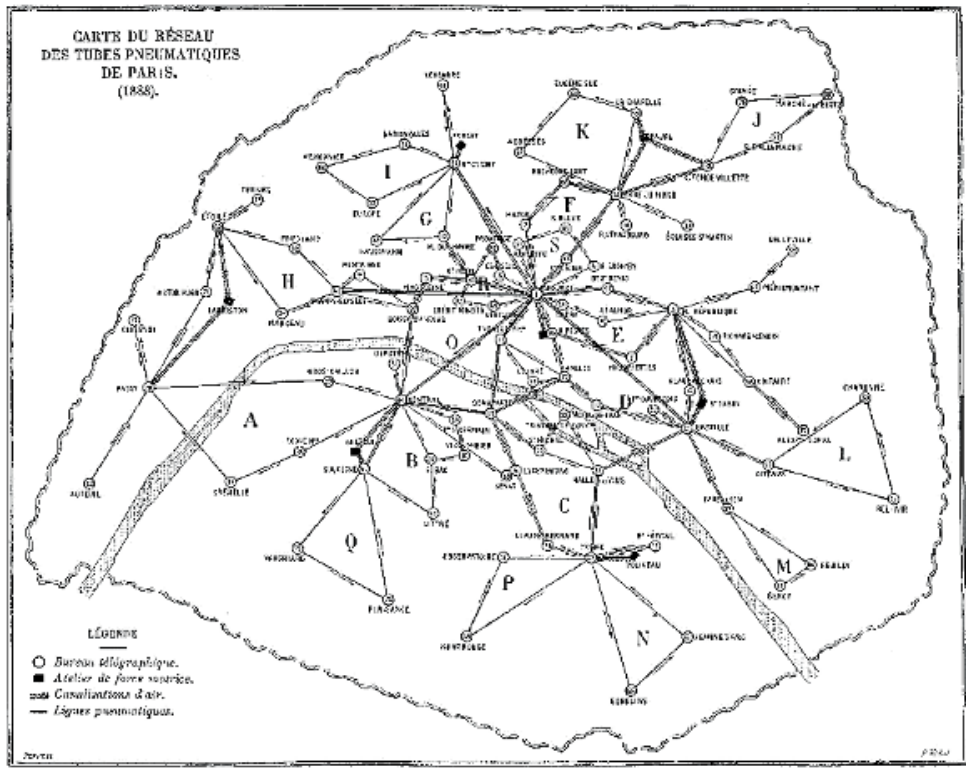

Abbildung 1: Schematisierung des Pariser Rohrpostnetzes aus dem Jahr 1888. Die mitunter großen Entfernungen zwischen den einzelnen Sende- und Empfangsstationen sind ersichtlich.

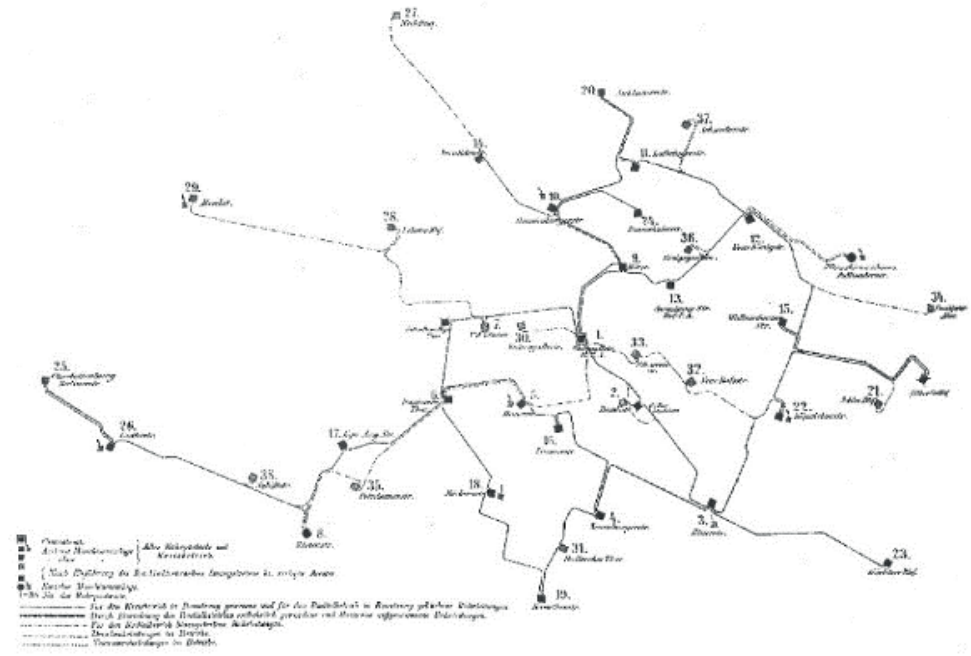

Abbildung 2: Schematisierung des Rohrpostnetzes von Berlin aus dem Jahr 1884. Es handelt sich hierbei ebenfalls um eine so genannte Fernrohrpostanlage. 


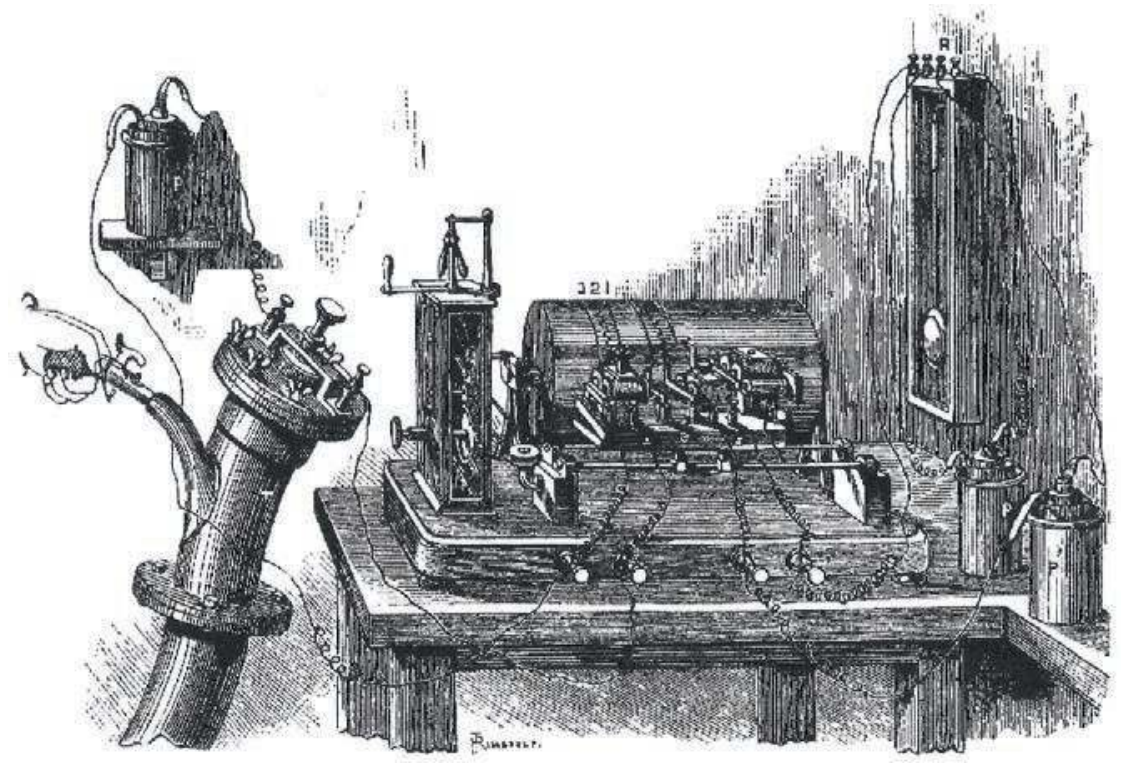

Abbildung 3: Grafische Darstellung von Charles Bontemps' »chronographischem Registriergerät« - die historisch erste Apparatur der aktiven Echoortung. 


\section{Christian Methfessel}

\section{Telegramme, Läufer, Brieftauben: Kommunikation in Zeiten imperialer Kriege}

\section{Einleitung}

Am 5. März 1898 traf ein Telegramm aus Sierra Leone im Colonial Office in London ein, das über die jüngste krisenhafte Entwicklung dort berichtete. Der Gouverneur der Kolonie, Frederic Cardew, informierte über die Kämpfe, die im >Hinterland « der Kolonie ausgebrochen waren: »I have received by carrier pigeon a report from Major Norris, Officer Commanding of the Troops, stating that he marched from Karene to Port Lokko yesterday, and stormed six villages and three fortified towns. “ ${ }^{1}$ In vier weiteren Sätzen wird kurz auf den Verlauf der Entwicklung nach Ausbruch des Konflikts eingegangen, der als >Hüttensteuerkrieg` in die britische Kolonialgeschichte eingegangen ist. ${ }^{2}$

Dass ein Kolonialgouverneur sich veranlasst sah, dem Kolonialminister in London, Joseph Chamberlain, zeitnah über den Verlauf der Gefechte an der Front zu berichten, zeigt wie sehr die Vernetzung der Welt durch Überseekabel und Telegraphie die politische Kommunikation am Ende des 19. Jahrhunderts verändert hatte. Politik und Öffentlichkeit erwarteten, schon am Folgetag über Ereignisse und die jüngste Entwicklung in weit entfernten Weltregionen informiert zu werden. Der Verweis auf den Empfang der Nachricht durch eine Brieftaube zeigt allerdings auch, dass die neue Technik der Telegraphie die älteren Kommunikationstechniken nicht vollständig verdrängt hatte. Die Grenzen der telegraphischen Nachrichtenübermittlung erforderten es vielmehr, die neue Technologie in Verbindung mit älteren Technologien zu nutzen. So war das telegraphische Netzwerk keinesfalls flächendeckend und Nach-

1 Governor Sir F. Cardew to Mr. Chamberlain (Received 12.30 a.m. March 5, 1898.) Telegram, National Archives, London (=NA), CO 879/55/1, Nr. 17, S. 19.

2 Vgl. Arthur Abraham: Nyagua, the British, and the Hut Tax War, in: The International Journal of African Historical Studies 5 (1972), S. 94-98; Ders., Bai Bureh, the British, and the Hut Tax War, in: The International Journal of African Historical Studies 7 (1974), S. 99-106. 
richten mussten in der Regel von einem Boten zur nächsten Telegraphenstation gebracht werden - oder wie in diesem Fall durch eine Brieftaube. Zudem war die telegraphische Nachrichtenübermittlung teuer, so dass solche Nachrichten meist kurz und skizzenhaft waren. ${ }^{3}$

Für die Kommunikation längerer Berichte war der Brief um 1900 zumeist alternativlos. Alterdings dauerte die Übermittlung von Briefen, gerade wenn sie von einem Kontinent $\mathrm{zu}$ einem anderen gebracht werden mussten, vergleichsweise lang. Das lässt sich auch an der Kommunikation zwischen dem Kolonialgouverneur in Sierra Leone und dem Minister in London aufzeigen. Neben dem kurzen Telegramm verfasste Ersterer am 5. März auch einen längeren Brief, in dem er ausführlich auf die Lage an der Front einging. Dieser Brief traf allerdings erst am 22. März ein, knapp zweieinhalb Wochen nach dem Telegramm. ${ }^{4}$

Dieses Nebeneinander alter und neuer Kommunikationstechnologien um 1900 wird im Folgenden anhand der Presseberichterstattung über Kolonialkriege skizziert. Der Blick auf die Medien erlaubt es dabei insbesondere, zu untersuchen, wie in Zeiten imperialer Krisen die Wege der Nachrichtenübermittlung das Bild beeinflussten, das man sich in Europa über weit entfernte Konflikte machte. Der Fokus wird auf das Britische Empire gelegt, zudem werden vereinzelt Seitenblicke auf das deutsche Kolonialreich geworfen. Abschließend wird dann nochmal ausführlich auf den eingangs erwähnten Krieg in Sierra Leone eingegangen. Dabei soll gezeigt werden, dass die Analyse der unterschiedlichen Formen der Kommunikation und ihres jeweils spezifischen timings entscheidend ist, um den Verlauf der politischen Kontroverse über den Ausbruch der Gewalt zu verstehen. Und Briefe spielten dabei weiterhin eine entscheidende Rolle.

3 Zur Geschichte der Telegraphie vgl. Daniel R. Headrick: The Invisible Weapon. Telecommunications and International Politics, 1851-1945, Oxford/New York 1991; Roland Wenzlhuemer: »Less Than No Time«. Zum Verhältnis von Telegrafie und Zeit, in: Geschichte und Gesellschaft 37 (2011), S. 592-613. Einen Eindruck der zunehmenden Vernetzung der Welt in der zweiten Hälfte des 19. Jahrhunderts vermittelt die dem Sammelband digital beigefügte »Chart of the World « (11. Aufl., 1886), auf der alle »Great Telegraph Lines« eingezeichnet sind. Die ausführliche Darstellung der "Steam Packet Communication«-Linien zeigt zudem die Wege des interkontinentalen Briefversands auf, der auch im Zeitalter der Telegraphie weiterhin essenziell für die globale Kommunikation war. Zur Chart of the World vgl. Iris Schröder, Eine Weltkarte aus der Provinz: Die Gothaer Chart of the World und die Karriere eines globalen Bestsellers, in: Historische Anthropologie 25 (2017), S. 353-376.

4 Governor Sir F. Cardew to Mr. Chamberlain (Received March 22, 1898.), NA, CO 879/55/1, Nr. 26, S. 29-33. 


\section{Nachrichtenübermittlung in Krisenzeiten: Kommunikationswege der Kriegsberichterstattung}

Die Dekaden nach 1880 gelten als »Zeitalter des Imperialismus«; die Kolonialmächte bauten ihren Herrschaftsbereich in der außereuropäischen Welt konstant aus, und die Konflikte zwischen ihnen um die >Aufteilung ‘ der international noch umstrittenen Territorien verschärften sich. ${ }^{5}$ Für die Presse in den imperialen Metropolen boten Nachrichten aus den Kolonien genau die Schlagzeilen, mit denen sich die Auflage steigern ließ. Insbesondere wenn es zum Ausbruch von Gewalt kam, ließen sich Ereignisse in der außereuropäischen Welt zu reißerischen Geschichten aufbauen. Gerade neue Zeitungsformate wie die 1896 auf den Markt gebrachte Daily Mail nutzten die Berichterstattung über Konflikte in den Kolonien, um eine stetig wachsende Leserschaft anzusprechen. ${ }^{6}$ So waren die imperiale Expansion und das rasante Wachstum der Presse am Ende des 19. Jahrhunderts eng miteinander verbunden. ${ }^{7}$

Die technologische Voraussetzung hierfür war die Verlegung von Unterseekabeln und Telegraphenleitungen in der zweiten Hälfte des 19. Jahrhunderts. Diese ermöglichten es, schon am Folgetag über die jüngste Entwicklung in anderen Kontinenten zu berichten. Wie schon erwähnt, war die telegraphische Übermittlung von Nachrichten jedoch sehr teuer. Korrespondenten mussten sich deswegen kurz fassen. Auch finanzstarke Medienunternehmen wie die Nachrichtenagentur Reuters setzten hier Grenzen. So instruierte Reuters ihren Berichterstatter während des britischen Krieges gegen den Sudan ${ }^{8}$ im November 1896 folgendermaßen:

»1. To send, if possible, a daily bulletin of 50 words reporting progress of the Expedition always prefixing the message by place and date of despatch, which must be telegraphed to us with the text of the message by the Retransmitting Agent.

5 Vgl. Jürgen Osterhammel: Die Verwandlung der Welt. Eine Geschichte des 19. Jahrhunderts, München 2009, S. $577 f$.

6 Vgl. Sally J. Taylor: The Great Outsiders. Northcliffe, Rothermere and the Daily Mail, London 1996, S. 36f., 44f.; Andrew Thompson: The Empire Strikes Back? The Impact of Imperialism on Britain from the Mid-Nineteenth Century, Harlow u.a. 2005, S. $241 f$.

7 Vgl. Christian Methfessel: Kontroverse Gewalt. Die imperiale Expansion in der englischen und deutschen Presse vor dem Ersten Weltkrieg, Köln/Weimar 2019, S. 133-140.

8 Die im März 1896 initiierte und Ende 1899 abgeschlossene militärische Kampagne gegen den Sudan verfolgten die englischen Zeitungen mit großem Interesse. Mediale Höhepunkte waren die Schlacht von Omdurman, bei der die Briten die sudanesische Hauptstadt einnahmen, und der diplomatische Konflikt mit Frankreich während der Faschoda-Krise im Herbst 1898, vgl. die medienhistorischen Beiträge in Edward M. Spiers (Hrsg.): Sudan. The Reconquest Reappraised, London/Portland 1998, sowie Simon J. Potter: Jingoism, Public Opinion, and the New Imperialism, in: Media History 20 (2014), S. 34-50. 
2. On the occasion of any very important fighting, to despatch, if possible, a short message giving bare result, so that we may get something through very quickly. In such cases the Correspondent will despatch to us a full account as speedily as possible afterwards.

3. In important cases the Correspondent's despatch may extend to 500 words. ${ }^{9}$

Als die Daily Mail zum Höhepunkt des >Boxerkriegs $\triangleleft{ }^{10}$ im Juli 1900 ein außergewöhnlich langes Telegramm nach Europa schickte, betonte die Zeitung die enormen Kosten, die damit verbunden waren. Allerdings waren die Umstände auch dramatisch. Zu dieser Zeit verfolgte man in Europa besorgt das Schicksal der im Pekinger Gesandtenviertel belagerten Europäerinnen und Europäer. Am 16. Juli schien es dann Gewissheit, dass alle Eingeschlossenen tot seien. Und der Shanghai-Korrespondent der Daily Mail hatte einen exklusiven Bericht über den genauen Tathergang:

»The complete account of the massacre at Pekin [sic!] which we publish this morning was despatched from Shanghai last night (Sunday) at 8 p.m. (Chinese time), and marked $>U r-$ gent. This meant that it travelled at the highest possible telegraphic cost, and reached us in almost record time, arriving at our offices at 7 p.m. (English time), Shanghai time being $7 \%$ hours ahead of ours. « ${ }^{11}$

Bei dem Bericht über das Massaker handelte es sich allerdings um eine Falschmeldung. Als die Truppen der alliierten imperialistischen Staaten gut einen Monat später Peking einnahmen, stellte sich heraus, dass die Belagerten (mit Ausnahme des deutschen Gesandten) noch am Leben waren. Damit zeigt diese Falschmeldung zum einen die Grenzen der telegraphischen Kommunikation um 1900 auf, zum anderen verdeutlicht sie, wie selbstverständlich die zeitnahe Berichterstattung aus Regionen wie China zu dieser Zeit zur Erwartungshaltung der Zeitungslesenden gehörte. Zuverlässige Berichte über die

9 Reuter's Telegram Company, Limited. Niger-Sudan Campaign. Instructions to Mr. William Wallace, Reuter's Correspondent. London, November 19th, 1896, Reuters Archive, London, Box LN 1016, 1/014435.

10 Bei den >Boxern ‘ handelte es sich um eine gegen die Präsenz der imperialistischen Staaten in China gerichtete Volksbewegung. Als diese sich im Sommer 1900 immer weiter ausbreitete, solidarisierte sich der chinesische Kaiserhof mit den >Boxern` und es kam zum Krieg zwischen China und den alliierten imperialistischen Staaten, der mit dem Friedensschluss im September 1901 (dem »Boxerprotokoll«) seinen Abschluss fand. Die europäischen Zeitungen verfolgten die Ereignisse in China gerade zu Beginn des Konflikts mit großer Spannung, vgl. Thoralf Klein: Propaganda und Kritik. Die Rolle der Medien, in: Mechthild Leutner, Klaus Mühlhahn (Hrsg.): Kolonialkrieg in China. Die Niederschlagung der Boxerbewegung 1900-1901, Berlin 2007, S. 173-180; Christian Methfessel: »Oxident gegen Orient«. Europabilder in der Berliner Morgenpost während des Boxerkriegs, in: Themenportal Europäische Geschichte (2009), https:// www.europa.clio-online.de/essay/id/fdae-1513 (letzter Zugriff: 08.08.2019).

11 The Pekin Massacre. All White Men, Women, and Children Put to the Sword. Awful Story of the 6th and 7th July. How Our People Died Fighting Prince Tuan's Hordes. Full Details From Our Special Correspondent, in: Daily Mail, Nr. 1321, 16.7.1900, S. 5. 
Situation der Belagerten in Peking waren jedoch schlicht nicht möglich. Die Telegraphenleitung nach Peking war von den chinesischen >Boxern` gekappt worden und es gelangten keine zuverlässigen Informationen nach Shanghai. So hatten die dort arbeitenden Korrespondenten nur die Möglichkeit, Spekulationen und Gerüchte wiederzugeben - was sie dann mit Blick auf das große Publikumsinteresse in Europa auch taten. ${ }^{12}$

Die Berichterstattung während des Boxerkriegs war sicherlich ein Extremfall. Aber zuverlässige Nachrichten vom Ort des Geschehens zu bringen war eine Herausforderung. So mahnte Reuters auch in Instruktionen aus dem Jahre 1898 zum schon erwähnten Sudankrieg mit Blick auf die erwarteten Berichte an:

»They, should, therefore, be compiled in the first person, and made as vivid, graphic, and interesting as possible, while not departing from proper sobriety of expression, and being careful to avoid mere inflation and extravagant rhetoric. Whenever the Correspondent may have to rely upon other parties for information of any fighting or other event, he should always endeavour to obtain the narrative of an eye-witness, or partaker in the affair, and give the same in the informant's own words. ${ }^{13}$

Sicherlich entsprachen nicht alle Kriegskorrespondenten dem in den Instruktionen geforderten Ideal, und ihre Artikel waren häufig von Übertreibungen geprägt. Darüber hinaus gaben die Berichterstatter immer wieder vor, als $\mathrm{Au}$ genzeugen vor Ort gewesen zu sein, selbst wenn ihre Ausführungen nur auf Gesprächen mit Beteiligten beruhten. ${ }^{14}$

Nicht nur zuverlässige Nachrichten von der Front zu bekommen, war eine Herausforderung, schließlich mussten diese auch vom Ort des Geschehens zur nächsten Telegraphenstation gebracht werden. Hierfür engagierten die Korrespondenten in der Regel Läufer. Dabei kam es immer wieder vor, dass Mitteilungen verloren gingen. Am schwierigsten war der Transport der Nachrichten wohl zu Beginn des `Burenkriegs`, den das Britische Empire um die Jahrhundertwende in Südafrika führte. ${ }^{15} \mathrm{Zu}$ Anfang des Krieges belagerten die

12 Vgl. Methfessel, Kontroverse Gewalt, S. 134, 144f., $183 f$.

13 Reuter's Telegram Company, Limited. Sudan Campaign (Autumn 1898). Instructions to Mr. Lionel James, Reuter's Correspondent. London, June 21st, 1898, Reuters Archive, London, Box LN 1016, 1/014435.

14 Vgl. Hugh Cecil: British Correspondents and the Sudan Campaign of 1896-98, in: Spiers (Hrsg.): Sudan, S. 102-127, hier S. 112f.; Stephen Badsey: War Correspondents in the Boer War, in: John Gooch (Hrsg.): The Boer War. Direction, Experience and Image, London/Portland 2000, S. 187-202, hier S. 190.

15 Bei den Buren handelte es sich um europäische Einwanderer im südlichen Afrika. Der Krieg des Britischen Empires gegen die Burenrepubliken Oranje-Freistaat und Transvaal (1899-1902) war neben dem >Boxerkrieg` das größte Medienereignis um die Jahrhundertwende, vgl. Kenneth Owen Morgan: The Boer War and the Media (1899-1902), in: 20th Century British History 13 (2002), S. 1-16. 
Buren mehrere Städte, unter den Eingeschlossenen befanden sich auch Zeitungskorrespondenten, die bestrebt waren, ihre Berichte nach England zu übermitteln. Häufig erreichten die eingesetzten afrikanische Läufer nicht ihr Ziel, sondern wurden auf ihrem Weg abgefangen, sodass die Mitteilungen verloren gingen und die Läufer sich der Gewalt der Buren ausgesetzt sahen. ${ }^{16}$

Jedoch musste nicht nur der erfolgreiche Transport der Nachrichten zur nächsten Telegraphenstation bewältigt werden, auch die Zensur durch britische Behörden bildete eine weitere Schranke, die es zu überwinden galt. Insbesondere die Besorgnis, dass Korrespondenten militärisch sensible Informationen verbreiteten würden, die die Buren für ihre Kriegsführung nutzen könnten, ließ eine strenge Zensur zwingend erscheinen. Aber auch Kritik ließ sich so unterdrücken. Korrespondenten übermittelten deswegen Mitteilungen, bei denen sie Zensur befürchteten, häufig per Brief nach England. Aufgrund der zeitlichen Verzögerung der Nachrichtenübermittlung auf diese Weise waren Briefe von der Zensur befreit und ermöglichten den Journalisten so größeren Spielraum. ${ }^{17}$

Während des Sudankriegs führte die Abhängigkeit der Journalisten von der militärischen Infrastruktur dazu, dass der Kriegsreporter Ernest Bennett seine scharfe Kritik an dem Feldzug erst übte, nachdem er nach England zurückgekehrt war. In einem Artikel, der im Januar 1899 in der Contemporary Review erschien, warf er britischen Soldaten vor, Verwundete getötet zu haben und berichtete von Plünderungen nach der Einnahme der sudanesischen Stadt Omdurman. Besonders kontrovers waren seine Ausführungen über die Schändung des Grabs des Mahdis, jenes religiösen Führers, der in den frühen 1880er Jahren die ägyptische Herrschaft über den Sudan beendet und den Staat, gegen den die Briten nun Krieg führten, aufgebaut hatte. ${ }^{18}$

Die Kommunikationstechniken, die für den Transport genutzt wurden, beeinflussten so nicht nur Länge und timing der Nachrichten, sie hatten auch entscheidenden Einfluss auf deren Inhalt. Mit Blick auf die Artikel der Kriegsberichterstatter waren Vorwürfe wie die von Ernest Bennett dennoch eine Ausnahme. Kritik von Korrespondenten beschränkte sich in der Regel auf Taktiken des militärischen Vorgehens, nur sehr selten wurde von dieser Seite die extrem brutale Kriegsführung in den Kolonien angeklagt. Bei den Kriegsreportern handelte es sich zumeist um ehemalige Militärs, die selbst überzeugte

16 Vgl. Badsey: War Correspondents in the Boer War, S. 191; Jacqueline Beaumont: The British Press During the South African War. The Sieges of Mafeking, Kimberley and Ladysmith, in: Mark Connelly, David Welch (Hrsg.): War and the Media. Reportage and Propaganda, 19002003, London/New York 2005, S. 1-18, hier S. 5-7, 11.

17 Vgl. Badsey, War Correspondents in the Boer War, bes. S. 196, $201 \mathrm{f}$.

18 Vgl. Cecil, British Correspondents, S. 107, $121 \mathrm{f}$. 
Anhänger der imperialen Expansion waren. Sie teilten Perspektive und Werte der Truppen, die sie begleiteten, und hatten grundsätzlich Verständnis für die ausgeübte Zensur, wenngleich sie häufig deren konkrete Umsetzung als zu streng ansahen. ${ }^{19}$

Dennoch unterschieden sich Telegramme und Briefe der Kriegskorrespondenten. Die kurzen Meldungen in den Telegrammen beschränkten sich zumeist auf die Mitteilung der wichtigsten Neuigkeiten und Entwicklungen. In Briefen - und noch mehr in den nach den Kriegen veröffentlichten Büchern hingegen finden sich auch ausführliche Landschaftsbeschreibungen und Beobachtungen über Menschen und Kultur der bereisten Krisenregionen. Zum britischen Krieg gegen Tibet ${ }^{20}$ erschien etwa am 27. August 1904 in der Times ein kurzer Artikel, der am 20. August in Lhasa verfasst wurde und von Konflikten zwischen britischen Truppen und einheimischer Bevölkerung in der eingenommenen Stadt berichtete. ${ }^{21}$ Zudem erschien am gleichen Tag ein sehr langer Bericht, in der Zeit vom 16. bis zum 22. Juli während des Vormarschs auf Lhasa verfasst, in dem der Korrespondent ausführlich das Himalaya-Gebirge beschrieb und dabei auch die Botanik der Region thematisierte und auf den "Aberglauben" der Tibeter einging. ${ }^{22}$ So trug die Kriegsberichterstattung zur Herstellung und Reproduktion von Selbst- und Fremdbildern bei, wobei die Korrespondenten vor allem Vorstellungen europäischer Überlegenheit verbreiteten und zur Aufrechterhaltung des imperialen Selbstverständnisses beitrugen. ${ }^{23}$

Der Zusammenhang zwischen den genutzten Kommunikationstechniken und den Bildern, die in Europa von Kolonialkriegen vermittelt werden konnten, ist auch mit Blick auf die Frage, welche Akteure Zugang zur Telegraphie

19 Vgl. ebd., S. 107-113; Andreas Steinsieck: Ein imperialistischer Medienkrieg. Kriegsberichterstatter im Südafrikanischen Krieg (1899-1902), in: Ute Daniel (Hrsg.): Augenzeugen. Kriegsberichterstattung vom 18. zum 21. Jahrhundert, Göttingen 2006, S. 87-112, hier S. 93-96.

20 Veranlasst durch die Sorge über den angeblich wachsenden Einflusses Russlands in Tibet, rückten Truppen Britisch-Indiens 1903 nach Lhasa vor und zwangen den Tibetern im September 1904 gewaltsam einen Handels- und Grenzvertrag auf. Die englische Presse verfolgte die Ereignisse mit einer Mischung aus Faszination und Zweifeln an der Sinnhaftigkeit des Militäreinsatzes, vgl. Glenn R. Wilkinson: `There is No More Stirring Story`: The Press Depiction and Images of War During the Tibet Expedition 1903-1904, in: War \& Society 9 (1991), S. 1-16; Methfessel, Kontroverse Gewalt, S. 261-166.

21 The British Mission to Tibet, in: The Times, Nr. 37484, 27.08.1904, S. 3.

22 With the Tibetan Mission. (From Our Special Correspondent.) The March to Lhasa, in: The Times, Nr. 37484, 27.8.1904, S. 10.

$23 \mathrm{Zu}$ europäischen Selbst- und Fremdbildern in der Berichterstattung über Kolonialkriege vgl. Christian Methfessel: Europa als Zivilisationsmacht. Kolonialkriege und imperialistische Interventionen in der deutschen und britischen Öffentlichkeit um 1900, in: Frank Bösch, Ariane Brill, Florian Greiner (Hrsg.): Europabilder im 20. Jahrhundert. Entstehung an der Peripherie, Göttingen 2012, S. 54-78. 
hatten, relevant. In der Regel konnten es sich nur die politischen und militärischen Vertreter der Kolonialmächte sowie die von Nachrichtenagenturen und finanzstarken Zeitungen entsandten Korrespondenten leisten, ihre Mitteilungen telegraphisch zu übermitteln, und bestimmten so die zeitnahe Berichterstattung über die Militäreinsätze. Eindrücke und Stellungnahmen anderer Akteure gelangten nur mit zeitlicher Verzögerung per Brief von den Krisenregionen nach Europa. Das trifft etwa auf die Briefe gewöhnlicher Soldaten zu, die an die Familie in der Heimat gerichtet waren, von den Familienmitgliedern aber zuweilen an lokale Zeitungen weitergereicht wurden. Während des Burenkrieg enthielten solche Briefe häufig Schilderungen von den Strapazen des Lebens an der Front, sodass auf diese Weise ein anderes Bild des Konfliktes ans Licht der englischen Öffentlichkeit kam als es die Berichte der Kriegskorrespondenten vermittelten. ${ }^{24}$ In deutschen Zeitungen erschienen während des Boxerkriegs und während des Kolonialkriegs in Südwestafrika ${ }^{25}$ Briefe, in denen beschrieben wurde, wie die eigenen Truppen Massaker an wehrlosen Zivilisten begingen. Für die Presse der oppositionellen Sozialdemokratie waren solche Briefe die Hauptquelle, um die Brutalität der deutschen Kriegsführung anzuklagen. ${ }^{26}$

So konnte das spätere Eintreffen von Nachrichten per Brief die Darstellungen von Kolonialkriegen in der europäischen Presse ändern und öffentliche Kontroversen auslösen. ${ }^{27}$ Im Folgenden soll das Zusammenspiel von telegraphischen und brieflichen Mitteilungen ausführlicher anhand der Debatte über die Ursachen des zu Beginn dieses Beitrags erwähnten Aufstands in der britischen Kolonie Sierra Leone 1898 analysiert werden.

24 Vgl. Brad Beaven: Visions of Empire. Patriotism, Popular Culture and the City, 1870-1939, Manchester u.a. 2012, S. 74-76.

25 Während der Niederschlagung der Aufstände der Herero und Nama in Südwestafrika (19041908) kam es zu einem selbst für die damaligen kolonialen Verhältnisse extrem brutalen Einsatz von Gewalt. Insbesondere die sozialdemokratische Presse kritisierte die genozidale Vorgehensweise der deutschen Truppen scharf, vgl. Methfessel, Kontroverse Gewalt, S. 266-288.

26 Vgl. Ute Wieland, Michael Kaschner: Die Reichstagsdebatten über den deutschen Kriegseinsatz in China: August Bebel und die »Hunnenbriefe«, in: Susanne Kuß, Bernd Martin (Hrsg.): Das Deutsche Reich und der Boxeraufstand, München 2002, S. 183-288; Merle Zeigerer: Kriegsberichterstatter in den deutschen Kolonialkriegen in Asien und Afrika. Augenzeugen, Anstifter, Komplizen?, Kiel 2016, S. 383.

27 Dieses Phänomen lässt sich schon in der Berichterstattung über den Indischen Aufstand 1857 beobachten, der erste Kolonialkrieg, der in Europa ein großes Medienereignis war, vgl. Tim Pratt: Ernest Jones' Mutinity. The People's Paper, English Popular Politics and the Rebellion 1857-58, in: Chandrika Kaul (Hrsg.): Media and the British Empire, Basingstoke/New York 2006, S. 88-103, hier S. $97 \mathrm{f}$. 


\section{Die Macht des Briefes: Die Debatte um den Kolonialkrieg in Sierra Leone}

Der Ausbruch der Gewalt in Sierra Leone 1898 muss im Kontext der sich wandelnden Kolonialpolitik in der zweiten Hälfte der 1890er Jahre verortet werden. Der 1895 ins Amt gekommene Kolonialminister Joseph Chamberlain maß den britischen Territorien in Westafrika eine hohe Bedeutung zu und unterstützte die koloniale Expansion ins Innere Afrikas. Gegen die britische Tradition setzte er sich für stärkeres staatliches Engagement und Investitionen in die Kolonien ein. Diese Politik der Expansion und Intensivierung der Kolonialherrschaft stieß vielerorts auf Widerstand. So kam es in der zweiten Hälfte der 1890er Jahre zu zahlreichen Kolonialkriegen in Westafrika, und regelmäßig thematisierten die Medien die dortigen Kriegsschauplätze. ${ }^{28}$

Ausbau und Ausweitung der Kolonialherrschaft und -verwaltung bildeten auch den Hintergrund für den Aufstand in Sierra Leone 1898, der in England eine Debatte über die richtige Administration im >Hinterland der Kolonie auslöste. Die britische Präsenz in Sierra Leone beschränkte sich lange Zeit auf das Küstengebiet. Hier siedelte das Empire in der Stadt Freetown und Umgebung befreite Sklaven an - diese und ihre Nachfahren werden in der Forschung zumeist >Kreolen` genannt, um zwischen ihnen und der afrikanischen Bevölkerung im Inneren Sierra Leones zu unterscheiden. Erst 1896 wurde über das >Hinterland ‘ der Kolonie ein britisches Protektorat deklariert. Der Gouverneur von Sierra Leone, Frederick Cardew, arbeitete zielstrebig daran, dort den britischen Einfluss auszubauen. Zur Finanzierung von Verwaltung und Infrastruktur führte er eine Hüttensteuer ein, die von Anfang 1898 an eingetrieben wurde. ${ }^{29}$

Im Februar stieß die Steuer im Norden des Protektorats auf Widerstand, der schließlich in einen gewaltsamen Konflikt mündete. Am 18. Februar fielen die ersten Schüsse, am Folgetag berichteten die Kommandierenden vor Ort hierüber an den Gouverneur. Da die eingangs erwähnten Brieftauben erst mit den später eintreffenden Verstärkungen zum Einsatz kamen, dauerte es, bis die Nachrichten aus Karina, einem Ort im Nordwesten Sierra Leones, von einem Boten in das ca. 55km Luftlinie entfernte Freetown gebracht wurden. Aber spätestens am 22. Februar war Cardew informiert; an diesem Tag telegraphier-

28 Vgl. Ronald Robinson, John Gallagher, Alice Denny: Africa and the Victorians. The Climax of Imperialism in the Dark Continent, New York/London 1961, S. 395-409.

29 Vgl. J. D. Hargreaves: The Establishment of the Sierra Leone Protectorate and the Insurrection of 1898, in: Cambridge Historical Journal 12 (1956), S. 56-80, hier S. 57-69. 
te er nach London über den Ausbruch der Gewalt, die Mitteilung traf dort um 21:00 Uhr ein. ${ }^{30}$

Damit war die Nachrichtenübermittlung von Sierra Leone nach England schneller als die Nachrichtenübermittlung von der Front zum Amtssitz des Gouverneurs. Die zeitnahe Berichterstattung an das Kolonialministerium war möglich, da Freetown seit 1886 direkt an das globale Telegraphennetzwerk angeschlossen war. ${ }^{31}$ Es dauerte dementsprechend nicht lange, bis auch die Zeitungen in London über die Ereignisse schrieben. Die Times berichtete erstmals am 25. Februar auf Basis eines Reuters-Telegramms aus Freetown vom Vortag über den Ausbruch von »serious disturbances «. ${ }^{32}$ Da keine Korrespondenten die Truppen begleiteten, waren die Kämpfe selbst kein großes Medienthema, es erschienen nur kurze Nachrichten auf Basis der Informationen, die von dem britischen Militär bereit gestellt wurden. Zudem war man besorgt über das Schicksal der im >Hinterland tätigen Missionare. ${ }^{33}$

Die eintreffenden Telegramme waren zumeist sehr kurz, es dauerte, bis Briefe aus der Krisenregion in England eintrafen. Dabei war der Personen- und Posttransport durch Dampfschiffe von Sierra Leone nach Europa schon seit einem knappen halben Jahrhundert gut etabliert. 1851 begannen Postdampfschiffe von England nach Südafrika auch in Freetown Station zu machen. Da sich diese Lösung als zu zeitaufwendig erwies, brachte ab 1852 ein Dampfschifffahrtslinie Pakete und Passagiere von mehreren westafrikanischen Häfen, darunter Freetown, zunächst nach Plymouth, ab 1858 nach Liverpool. In der Folgezeit nahm der Schiffsverkehr zwischen Westafrika und Europa stetig zu. Zudem sanken die Preise, sodass sich ein größer werdender Personenkreis, darunter auch kreolische Händler, die Reise von Afrika nach Europa leisten konnte. ${ }^{34}$

30 Vgl. Governor Sir F. Cardew to Mr. Chamberlain (Received 9 p.m., February 22, 1898.), Telegram, NA, CO 879/55/1, Nr. 15, S. 18; Governor Sir F. Cardew to Mr. Chamberlain (Received March 19, 1898.), NA, CO 879/55/1, Nr. 25, S. 22-29; Christopher Fyfe: A History of Sierra Leone, London u.a. 1962, S. 561-563. Vgl. auch Abraham, Bai Bureh, demzufolge der Ausbruch des militärischen Konfliktes auf britische Initiative zurückging.

31 Vgl. Fyfe, A History of Sierra Leone, S. 457f. In der digital beigefügten Chart of the World aus dem Jahre 1886 ist der telegraphische Anschluss Freetowns noch als "projected « eingetragen.

32 Sierra Leone, in: The Times, Nr. 35449, 25.2.1898, S. 5.

33 Vgl. etwa The Fighting in Sierra Leone, in: The Times, Nr. 35459, 9.3.1898, S. 5; Sierra Leone, in: The Times, Nr. 35477, 30.3.1898, S. 7.

34 Vgl. Fyfe, A History of Sierra Leone, S. 266, 415, 445, 458, 478. Die Dampfschifffahrtslinie zwischen Freetown und Liverpool sowie die Verbindungen zwischen Westafrika und Europa allgemein sind in der digital beigefügten Chart of the World aufgeführt. 
Trotz der kontinuierlichen Fortschritte in der Schifffahrtstechnik ${ }^{35}$ sowie dem regelmäßigen Verkehr zwischen Sierra Leone und England vergingen Wochen, bis Briefe aus der Kolonie in London eintrafen. So verfasste Gouverneur Cardew nach Ausbruch des Aufstands nicht nur ein Telegramm am 22. Februar, drei Tage später schrieb er zudem einen ausführlichen Brief an Chamberlain. Dieser traf jedoch erst am 19. März, also gut drei Wochen später, im Kolonialministerium ein. ${ }^{36}$

Die Times veröffentlichte erstmals am 9. April einen Brief, in dem ein Korrespondent ausführlicher auf den bisherigen Verlauf der Ereignisse einging. Dort kann man etwa zum Einsatz von Brieftauben lesen:

"For some days no news was received in Freetown of how the expedition was faring. It was after a time rumoured that some sharp fighting had taken place, and at last rumour was resolved into fact by the arrival of some carrier pigeons which had been taken with the expedition. These birds, which have so far been the only rapid and reliable means of communication between the troops and the town, belong, it is understood, to a merchant in Freetown who uses them in his business with the interior, and who placed them at the disposal of the Government on the present occasion. « ${ }^{37}$

Ein weiterer ausführlicher Artikel erschien dann am 13. April auf Basis von Briefen und Berichten von Passagieren, die am Vortrag mit dem Dampfschiff Axim in Liverpool eingetroffen waren. Dem Artikel zufolge stammten die letzten von Brieftauben übermittelten Nachrichten vom 26. März, zwei Tage bevor die Axim von Sierra Leone nach Europa aufgebrochen war. ${ }^{38}$

Ende April weitete sich der Aufstand dann auf den Süden des Protektorats aus, und die Nachrichten wurden dramatischer. ${ }^{39}$ Die Daily Mail schrieb unter der Überschrift Bloodshed in Sierra Leone am 19. Mai: »The whole of the natives in the Sherbro district, it was stated, were in open rebellion, and had butchered over 200 people, and destroyed all the up-river factories. $\aleph^{40} \mathrm{Die}$ Presse erörterte die Gefahr für Freetown und berichtete über Massaker an

35 Vgl. Daniel Richard Headrick: The Tools of Empire. Technology and European Imperialism in the Nineteenth Century, New York/Oxford 1981, S. 129-149.

36 Governor Sir F. Cardew to Mr. Chamberlain (Received March 19, 1898.), NA, CO 879/55/1, Nr. 25, S. 22-29.

37 The Disturbances in Sierra Leone, in: The Times, Nr. 35486, 9.4.1898, S. 4. Das Schreiben ist leider undatiert, Länge wie Inhalt lassen jedoch schlussfolgern, dass es sich um einen Brief handelt.

38 Events in Sierra Leone and Lagos, in: The Times, Nr. 35489, 13.4.1898, S. 8.

39 Nach Abraham, Nyagua, gingen die britischen Truppen im Süden auch gegen Afrikaner vor, die sich eigentlich nicht am Aufstand beteiligt hatten und trugen so zur weiteren Eskalation der Gewalt bei.

40 Bloodshed in Sierra Leone, in: Daily Mail, 19.5.1898, S. 3. 
amerikanischen Missionaren. ${ }^{41}$ Aber schon Mitte Juni gelang es den Truppen des Empires, die Lage unter Kontrolle zu bringen, auch wenn sich die Kämpfe im Protektorat noch bis Ende 1898 hinzogen. ${ }^{42}$ Die Situation an der Front bildete allerdings nicht den Fokus des Medieninteresses. Die Diskussionen drehten sich vielmehr um die Ursachen des Aufstandes.

Die Debatte über die politische Verantwortung für den Konflikt begann recht spät. Als sich Mitte April herausstellte, dass die Lage ernster war als zunächst vermutet, erschienen vereinzelt Kommentare in der Presse hierzu. Und erst im Mai begannen die Zeitungen, sich ausführlicher der Frage zu widmen, wer Schuld an der Eskalation der Gewalt hatte. Dabei herrschte zunächst Konsens, dass die Einführung der Hüttensteuer ein politischer Fehler gewesen war und die Kolonialadministration dementsprechend die Verantwortung für die Entwicklung trug. Diese Interpretation schien insbesondere schlüssig, da die Manchester und Liverpooler Handelskammern sowie die Afrikareisende Mary Kingsley sich schon zuvor öffentlich gegen die Einführung der Hüttensteuer positioniert hatten. In einem Treffen der Manchester Handelskammer, über das der Manchester Guardian am 14. April 1898 berichtete, erinnerten Redner daran, dass sie schon im Sommer des Vorjahres vor der Einführung der Hüttensteuer gewarnt hatten, aber im Kolonialministerium auf kein Verständnis gestoßen seien. ${ }^{43}$ Am Folgetag kommentierte der Guardian daraufhin:

»[C]ommercial bodies, as well as travellers like Miss Kingsley, quite expected the disturbances which have resulted. The collection of the tax has been ruinously expensive. [...] The Government of the colony must surely withdraw an impost at once ruinous to itself and obnoxious to the natives. ${ }^{44}$

Nicht nur der linksliberale Guardian, auch die konservative Pall Mall Gazette urteilte in diesem Sinne. Am 5. Mai veröffentliche sie ein Interview mit einem Liverpooler Händler, der die Hüttensteuer als »unjust and tyrannical policy" bezeichnete und sich für die Abberufung des Gouverneurs aussprach. ${ }^{45}$ Einen Tag später urteilte die Pall Mall Gazette dann selbst:

41 Vgl. The Troubles in Sierra Leone, in: The Times, Nr. 35508, 5.5.1898, S. 5; The Rising in Sierra Leone, in: The Times, Nr. 35509, 6.5.1898, S. 5; The Rising in Sierra Leone, in: The Times, Nr. 35510, 7.5.1898, S. 7.

42 Vgl. Hargreaves, The Establishment of the Sierra Leone Protectorate, S. 72.

43 Manchester Chamber of Commerce, in: The Manchester Guardian, Nr. 16123, 14.4.1898, S. 10.

44 O.T., in: The Manchester Guardian, Nr. 16124, 15.4.1898, S. 5.

45 The Hut-Tax Question in West Africa. [From a Liverpool Correspondent.], in: The Pall Mall Gazette, Nr. 10329, 5.5.1898, S. 3. 
»We are always reluctant to be hasty in saying anything that reflects upon a British official at his post in a distant land, but there can be no doubt that the hut-tax was a bad mistake, or that the Governor has been going distinctly too fast. « ${ }^{46}$

Etwas vorsichtiger argumentierte die regierungsnahe Times. Für sie war der Aufstand eine Folge der Einführung >zivilisierter` Herrschaft in einem >barbarischen`Gebiet. Aber die Times schloss nicht aus, dass die Hüttensteuer die falsche Methode gewesen sei, um den Aufbau der britischen Verwaltung zu finanzieren, und hielt es für notwendig, über Alternativen nachzudenken. ${ }^{47}$

Auch Kolonialminister Chamberlain sah zunächst im Einklang mit der öffentlichen Meinung in der Hüttensteuer die Ursache für den Ausbruch des Aufstands in Sierra Leone, wenngleich er sich mit öffentlichen Stellungnahmen in diesem Sinne zunächst zurückhielt. ${ }^{48}$ Die Kritik der Presse setzte ihn aber zunehmend unter Druck, sich in dieser Frage zu positionieren. Zudem richteten die in Sierra Leone aktiven Händler Eingaben direkt an das Colonial Office, in denen sie ihre Kritik an der Hüttensteuer wiederholten. Einem Schreiben der Liverpooler Handelskammer vom 15. April etwa waren noch mehrere Briefe aus Sierra Leone beigelegt, in denen Gouverneur Cardew und seine Politik scharf angegriffen wurden. ${ }^{49}$ Eine Eingabe der Manchester Handelskammer vom 2. Mai forderte Chamberlain mit Verweis auf die aus Sierra Leone erhaltenen Telegramme und Briefe dazu auf, Cardew zu instruieren, die Einziehung der Hüttensteur unverzüglich zu suspendieren. ${ }^{50}$ In Reaktion auf die anhaltende Kritik telegraphierte Chamberlain am 3. Mai an Cardew:

»In view of questions in House of Commons, report fully by telegraph your views as to situation. Merchants in the Colony are telegraphing to correspondents here situation of affairs serious, entirely due to hut tax imposed contrary to local advice, and ought to be withdrawn. It is evident that policy must be reconsidered; have you any suggestions? « ${ }^{51}$

Cardew erbat sich am Folgetag Zeit und versprach eine Antwort, sobald sich die Lage beruhigt habe. ${ }^{52}$ Damit gab sich Chamberlain allerdings nicht zufrieden; in einem weiteren Telegramm vom 6. Mai empfahl er dem Gouverneur, öffentlich zu erklären, dass er die Einwände gegen die Eintreibung der Hütten-

46 O.T., in: The Pall Mall Gazette, Nr. 10330, 6.5.1898, S. 2.

47 The Colonies. The Disturbances in Sierra Leone, in: The Times, Nr. 35511, 9.5.1898, S. 12.

$48 \mathrm{Vgl}$. Hargreaves, The Establishment of the Sierra Leone Protectorate, S. 73.

49 Liverpool Chamber of Commerce to Colonial Office (Received April 16, 1898.), NA, CO $879 / 55 / 1$, Nr. 46, S. 57-59.

50 Manchester Chamber of Commerce to Colonial Office (Received May 3, 1898.), NA, CO/879/55/1, Nr. 63, S. 71.

51 Mr. Chamberlain to Governor Sir F. Cardew .(Sent 1.15 p.m., May 3, 1898.) Telegram, NA, CO 879/55/1, Nr. 64, S. 72.

52 Governor Sir F. Cardew to Mr. Chamberlain. (Received 10.35 a.m., May 4, 1898.) Telegram, NA, CO 879/55/1, Nr. 65, S. 72. 
steuer in dieser Höhe anerkenne und deren Einziehung vorab suspendiere. ${ }^{53}$ Daraufhin verfasste Cardew am 9. Mai ein Telegramm, in dem er der Kritik der Händler widersprach. Diese argumentierten nur aus ihrem Eigeninteresse heraus und wären an anderen Ursachen des Aufstandes als der Hüttensteuer nicht interessiert, darin würden sie sich von den Missionaren unterscheiden. Die Situation sei von den Händlern und der Presse in Freetown angeheizt worden, die gegen die Hüttensteuer agitiert hätten. Damit gab er den Kreolen die Schuld am Ausbruch der Gewalt. Tatsächlich wäre es für die Bewohner im Protektorat ein Leichtes, die Hüttensteuer zu bezahlen. ${ }^{54}$

Das Telegramm traf vormittags ein, und als Chamberlain sich am Nachmittag des 9. Mai im House of Commons zu den Ereignissen in Sierra Leone äußerte, sprach er sich gegen eine voreilige Verurteilung des Gouverneurs aus. Im Einklang mit Cardew verurteilte er die Agitation der Kreolen und beschrieb mit Verweis auf einen Brief des Bischofs von Sierra Leone den Anführer der Aufständischen als »drunken slaver«. Zur Fortsetzung der Hüttensteuer wollte er sich jedoch nicht festlegen und erklärte, dass ein Special Commissioner nach Sierra Leone entsandt werde, um die Ursachen des Aufstands zu untersuchen. ${ }^{55}$ Unterstützung bekam Cardew zudem von Graham Ingham, dem ehemaligen Bischof von Sierra Leone, der den Gouverneur in einem Brief verteidigte, den die Times am 12. Mai veröffentlichte. Für ihn war die Entsendung eines Special Commissioner unnötig; im Einklang mit Cardew wies er in dem Brief der Freetown Presse die Schuld zu. Dafür berief er sich auf seine langjährige Erfahrung mit dem illoyalen Charakter der Kreolen, räumte aber - inzwischen wieder in England lebend - ein, dass er die vor Ausbruch des Aufstands verfassten Artikel nicht kenne. ${ }^{56}$

Nachdem zunächst klar schien, dass die Einführung der Hüttensteuer den Konflikt verursacht habe, war es inzwischen wieder eine offene Frage, wem die Schuld am Lauf der Dinge zuzuschreiben sei. Auch die Pall Mall Gazette urteilte nun: »Bishop Ingram's letter to the Times throws a good deal of light on the Sierra Leone business, and makes strongly for the view that there is

53 Mr. Chamberlain to Governor Sir F. Cardew. (Sent 3.15 p.m., May 6, 1898.) Telegram, NA, CO 879/55/1, Nr. 72, S. 78.

54 Governor Sir F. Cardew to Mr. Chamberlain. (Received 9.50 a.m., May 9, 1898.) Telegram, NA, CO 879/55/1, Nr. 78, S. 80. Am 10. Mai ergänzte Cardew in einem Folgetelegramm, dass der wahre Grund für den Aufstand nicht die Hüttensteuer, sondern die Unterdrückung des Sklavenhandels im >Hinterland d durch das Empire sei, Governor Sir F. Cardew to Mr. Chamberlain. (Received 10.54 a.m., May 10, 1898.) Telegram, NA, CO 879/55/1, Nr. 81, S. 82.

55 Zit. nach Parliament, in: The Times, Nr. 35512, 10.5.1898, S. 8-9, Zitat S. 8.

56 E. Graham Ingham, Sierra Leone. To the Editor of the Times, in: The Times, Nr. 35514, 12.5.1898, S. 7. Im August veröffentlichte die Times eine Erwiderung des Machers der Sierra Leone Weekly News, vgl. C. May, The Sierra Leone Rising. To the Editor of the Times, in: The Times, Nr. 35589, 8.8.1898, S. 4. 
another side to the complaints against Sir F. Cardew's action." Im Gegensatz zu Ingram hielt die Pall Mall Gazette die Entsendung eines Special Commissioner jedoch weiterhin für nötig. Man müsse prüfen, ob die Einführung der Steuer klug gewesen sei, und ob man sie beibehalten werde. ${ }^{57}$

Tatsächlich sollten sich entscheidende Akteure schon ihre Meinung bilden, bevor es zur Entsendung des Special Commissioner kam. Ausschlaggebend dafür war, dass im Juni Briefe eintrafen, die den Deutungswandel bestärkten, der sich schon im Mai abgezeichnet hatte. Nach seinem Telegramm verfasste Cardew am 28. Mai einen längeren Brief, der am 13. Juni in London eintraf. In diesem wiederholte er ausführlich seine Kritik an der Freetown Presse und den Händlern. Zur Rolle der Zeitungen schrieb er:

»[I]t is a well-known fact that these journals are circulated amongst them [die >chiefs $<-$ $\mathrm{CM}]$ and interpreted to them by the English-speaking clerk whom each chief of any importance has on his staff."

Zur Rolle der Händler äußerte er sich ebenso kritisch: Diese hätten nur ihre kurzfristigen ökonomischen Interessen im Auge und stünden der Entwicklung des Protektorats gleichgültig gegenüber. So habe sich ein Händler beschwert, dass die Abschaffung der Sklaverei schlecht für sein Geschäft sei. Ein anderer habe Schießpulver an die Bewohner des `Hinterlandes` verkauft. In den Augen Cardews war der Aufstand vor allem eine Rebellion der >chiefs‘, die durch die Abschaffung der Sklaverei ihre traditionelle Autorität in Gefahr sähen. Die Hüttensteuer sei nur ein Vorwand gewesen. Stellungnahmen aus Missionskreisen, die auf Bitte Cardews hin verfasst wurden und dem Brief beilagen, unterstützten diese Interpretation. ${ }^{58}$ Mit diesem Brief schaffte Cardew es, Chamberlain von seiner Position zu überzeugen. Bevor der zum Special Commissioner ernannte David Chalmers seine Reise nach Sierra Leone antrat, wurde er von Chamberlain ermahnt, nicht zu hart über die Hüttensteuer zu urteilen. ${ }^{59}$

Auch die Times positionierte sich in diesem Monat eindeutig auf der Seite Cardews. In einem längeren Artikel argumentierte ein Korrespondent am 27. Juni, dass die Rolle der Hüttensteuer überschätzt werde. Der Aufstand richte sich hauptsächlich gegen die Bekämpfung von Sklaverei und Kannibalismus im

57 O.T., in: The Pall Mall Gazette, Nr. 10335, 12.5.1898, S. 2.

58 Governor Sir F. Cardew to Mr. Chamberlain. (Received June 13, 1898.), NA, CO 879/55/1, Nr. 100, S. 112-127. Die Vorwürfe gegenüber der Freetown Presse dienten vor allem dazu, auf einen Sündenbock für den Ausbruch des Aufstandes zeigen zu können. Tatsächlich riefen die Artikel zur Hüttensteuer bei aller Kritik nicht zu Gewalt gegen die britische Herrschaft auf. Auch die Angaben Cardews zur Praxis der Zeitungslektüre unter den >chiefs` sind wohl übertrieben, wobei es durchaus Belege dafür gibt, dass Zeitungen im `Hinterland`Sierra Leones zirkulierten, vgl. Fyfe, A History of Sierra Leone, S. 579.

59 Daniel R. Magaziner, Removing the Blinders and Adjusting the View: A Case Study from Early Colonial Sierra Leone, in: History in Africa 34 (2007), S. 169-188, hier S. 180. 
britischen Protektorat und sei durch den »evil influence of the Freetown Press« ermutigt worden. ${ }^{60}$ In einem Kommentar am gleichen Tag verglich die Times die Kritik an der Hüttensteuer mit den Ausführungen des Korrespondenten und schloss sich der letzteren Position an. Die Unruhen in Sierra Leone seien "one more instance of the resistance which barbarous social organism everywhere offer to the introduction of the first rudiments of order and justice.«Zur Presse der Kreolen schrieb sie:

»Not the least interesting part of Sir David Chalmer's report will be that which deals with the conduct of the inhabitants of the colony proper, whom Mr. Chamberlain accuses of inciting the chiefs to resist the Government of the Queen. ${ }^{61}$

Chalmers sollte die Erwartungen Chamberlains und der Times enttäuschen. Für ihn war die Hüttensteuer unzweideutig Ursache des Aufstandes. Allerdings sollte es dauern, bis sein Bericht erschien. Chalmers erreichte Freetown im Juli 1898 und blieb dort für vier Monate, um Zeugen zu befragen. Im Januar 1899 reichte er einen Bericht beim Colonial Office ein, in dem er die Kolonialverwaltung für den Aufstand verantwortlich machte. Nach kurzem Schwanken entschied Chamberlain sich, Cardew die Möglichkeit zu einer Gegendarstellung einzuräumen. Den beiden Stellungnahmen fügte das Colonial Office einen dritten Teil hinzu, als dessen Autor Chamberlain angegeben wurde und der in den wesentlichen Punkten die Position Cardews unterstützte. ${ }^{62}$

In der Presse erregte die Kritik an der Hüttensteuer nur noch begrenztes Aufsehen, als der Bericht schließlich im Juli 1899 veröffentlicht wurde. Die Manchester und Liverpooler Handelskammer waren zu diesem Zeitpunkt schon auf Linie gebracht und hielten sich in der Öffentlichkeit zurück. ${ }^{63} \mathrm{Er}$ wartungsgemäß bezog die Times in ihrem Kommentar am 27. Juli Stellung im Sinne Cardews und Chamberlains. ${ }^{64}$ Für die Pall Mall Gazette zeigte der Bericht von Chalmers zwar, dass Cardew mit der Hüttensteuer zu überstürzt

60 The Rising in Sierra Leone. (From a Correspondent), in: The Times, Nr. 35553, 27.6.1898, S. 4. Die Ausführungen sind im Wesentlichen auf der Linie des Briefes, den Cardew an Chamberlain richtete. Einige Details - etwa die zur Architektur der Hütten im Protektorat - finden sich im fast gleichen Wortlaut in beiden Texten, sodass man vermuten kann, dass der Artikel zumindest unter Einwirkung von Cardew in Sierra Leone verfasst wurde oder der Text über den Umweg des Colonial Office seinen Weg von Sierra Leone in die Redaktion der Times fand. Für die zweite Vermutung spricht auch, dass das Colonial Office im Oktober 1898 - ein Zeitpunkt, zu dem die Arbeit des Special Commissioners noch nicht abgeschlossen war - einen Bericht an die Times weitergab, der Cardews Politik verteidigte, vgl. Magaziner, Removing the Blinders, S. 178. 61 The Disturbances in Sierra Leone, in: The Times, Nr. 35553, 27.6.1898, S. 11.

62 Vgl. Hargreaves, The Establishment of the Sierra Leone Protectorate, S. 75-79.

63 Darauf verwies ein Gastbeitrag, der kurz vor Erscheinen des Berichtes in der Pall Mall Gazette erschien. Der Autor des Beitrags hielt dessen ungeachtet an der Kritik an der Hüttensteuer fest, vgl. E.D.M., The Hut-Tax in West-Africa, in: The Pall Mall Gazette, Nr. 10709, 24.7.1898, S. 1f. 64 The Sierra Leone Papers, in: The Times, Nr. 35892, 27.7.1898, S. 9. 
vorgegangen sei. Insgesamt hielt sie den Kurs von Chamberlain, die Hüttensteuer fortzuführen, aber in Einzelfällen ganz oder teilweise erlassen zu können, für vernünftig. ${ }^{65}$

Der Manchester Guardian sah in dem Report Chalmers eine eindeutige Verurteilung der Kolonialpolitik in Sierra Leone und den Beweis, dass der Aufstand auf die Hüttensteuer zurückzuführen sei. Er griff Chamberlain an, dass er den Bericht wie "waste paper « behandle, und urteilte, dass die Untersuchung nur ein Trick gewesen sei, damit die Öffentlichkeit den Skandal vergesse: »But the matter cannot be allowed to end here. ${ }^{66}$ Doch die Hüttensteuer wurde nicht abgeschafft. ${ }^{67}$ Anfang August konnte Chamberlain zudem die »Colonial Loans Bill« im House of Commons durchbringen, die größere Investitionen in Westafrika vorsah, unter anderem für eine dritte Eisenbahn in Sierra Leone. Die kolonialfreundliche Stimmung zu dieser Zeit zeigte sich nicht zuletzt darin, dass das Gesetz selbst bei den oppositionellen Liberalen nur auf begrenzten Widerstand stieß. ${ }^{68}$

Dass es Chamberlain im Sommer 1899 gelang, seinen Kurs des stärkeren staatlichen Engagements in Westafrika durchzusetzen, hing auch mit der allgemeinen Stimmung zu dieser Zeit zusammen. Als der Aufstand in Sierra Leone im Frühjahr 1898 ausbrach, schien die Lage an mehreren Fronten des britischen Kolonialreichs kritisch. Im gleichen Jahr kam es in mehreren afrikanischen Kolonien zum Ausbruch von Gewalt; an der indischen Grenze sah sich das Empire immer noch mit einem größeren Aufstand konfrontiert, der schon im Sommer des Vorjahres ausgebrochen war; der Feldzug in den Sudan bereitete zwar aus britischer Perspektive weniger Sorgen, aber mit Blick auf einen möglichen Konflikt mit Frankreich war die Lage durchaus brenzlig; und in China setzten die Expansionsbestrebungen Deutschlands und Russlands das Empire unter Druck. Im Sommer 1899 hingegen war die Lage an der indischen Grenze unter Kontrolle gebracht; die Presse feierte den Sudanfeldzug als großen Erfolg und London ging aus der Faschoda-Krise als Sieger im diplomatischen Konflikt mit Paris hervor; insgesamt schienen die Aussichten für eine aktivere Imperialpolitik nun weitaus vielversprechender zu sein. ${ }^{69}$ Damit hatte sich die Taktik Chamberlains, auf Zeit zu setzen und die Entscheidung über die Hüttensteuer über ein Jahr hinaus zu verzögern, letztlich bewährt.

65 O.T., in: The Pall Mall Gazette, Nr. 10712, 27.7.1898, S. 2.

66 O.T., in: The Manchester Guardian, Nr. 16525, 27.7.1898, S. 7.

67 Vgl. Hargreaves, The Establishment of the Sierra Leone Protectorate, S. 79f.

68 Vgl. Robinson, Gallagher, Denny, Africa and the Victorians, S. 400-402. Ein Jahr zuvor hatte

Chamberlain das Gesetz in Anbetracht starker Opposition zurückziehen müssen.

69 Vgl. Hargreaves, The Establishment of the Sierra Leone Protectorate, S. 72; Methfessel, Kontroverse Gewalt, S. 116-119. 


\section{Fazit}

Die Kontroversen um den Hüttensteuerkrieg in Sierra Leone zeigen, wie sehr das timing bestimmter Beiträge und Entscheidungen sowohl Verlauf als auch Ausgang der Debatte beeinflussten. Um dieses timing zu verstehen, ist es wichtig, die Kommunikationswege von der Krisenregion zur imperialen Metropole nachzuvollziehen. Als der Aufstand im Frühjahr 1898 ausbrach, bestimmten die Handelskreise in Manchester und Liverpool die Interpretation der Ereignisse in der englischen Öffentlichkeit. Sie hatten die Einführung der Hüttensteuer schon zuvor abgelehnt und konnten sich in ihren Stellungnahmen auf in Sierra Leone tätige Händler berufen. Die allgemein als krisenhaft wahrgenommene Situation im Empire trug zusätzlich dazu bei, dass eine solche Deutung Wirkungsmacht entfalten konnte. Auch konservative Zeitungen wie die Pall Mall Gazette und sogar der Kolonialminister selbst hinterfragten zu diesem Zeitpunkt die Weisheit der in Sierra Leone verfolgten Politik.

Es dauerte, bis der Gouverneur vor Ort begriff, wie gefährlich die Debatten in England für seine Politik - und seine Position - waren. Dann schaffte er es jedoch in Zusammenarbeit mit Verbündeten aus Missionskreisen erfolgreich, die Debatte in seinem Sinne zu beeinflussen. Mit den kurzen Telegrammen, die er zunächst verschickte, konnte er sich allerdings nur etwas Zeit erkaufen. Entscheidend waren die längeren Briefe, die ab Juni in London eintrafen und die Politik des Gouverneurs verteidigten. So konnte zumindest in prokolonialen Kreisen eine Deutung der Ereignisse durchgesetzt werden, nach der nicht die Hüttensteuer, sondern Widerstand gegen die Unterdrückung des Sklavenhandels und die Illoyalität der Kreolen die eigentlichen Ursachen des Aufstandes waren. Die Entsendung eines Special Commissioner hatte dann kaum noch Auswirkungen auf die Debatte. Seine Verurteilung der Hüttensteuer bewegte niemanden in England mehr, die einmal eingenommene Position zu ändern. Ausschlaggebend für den Ausgang der Kontroverse war nur, dass der Bericht des Special Commissioner erst im Sommer 1899 veröffentlicht wurde - zu einem Zeitpunkt, als der Aufstand in Sierra Leone längst niedergeschlagen war und die Situation im Empire allgemein weniger kritisch schien als noch ein Jahr zuvor. Unter diesen Umständen gelang es Chamberlain schließlich, seinen kolonialen Kurs erfolgreich in der englischen Politik durchzusetzen.

Dass eine zunächst kritische öffentliche Meinung durch Briefe auf den kolonialen Kurs der Regierung gebracht wurde, war allerdings in der Zeit um 1900 eher selten der Fall. Die öffentlichen Beiträge der Manchester und Liverpooler Handelskammern, die schon zuvor die Politik des Gouverneurs in Sierra Leone abgelehnt hatten und regelmäßig mit Händlern vor Ort kommunizierten, führten dazu, dass sich zunächst eine äußerst regierungskritische Posi- 
tion durchsetzen konnte. Zumeist stammten die ersten Nachrichten, die telegraphisch aus den Krisenregionen nach Europa gelangten, von den politischen oder militärischen Vertretern der Regierung in der Kolonie. Auch wenn Zeitungskorrespondenten vor Ort waren, nahmen diese in der Regel einen ähnlichen Blickwinkel ein und unterstützten die imperiale Expansion ihres Landes. Abweichende Stimmen kamen nur mit Verzögerung per Brief nach Europa. Dies war etwa während der deutschen Kolonialkriege in China und Südwestafrika der Fall, als Briefe gewöhnlicher Soldaten der sozialdemokratischen Presse als Beleg dienten, um die brutale Kriegsführung der Truppen des Kaiserreichs anzuklagen.

In jedem Fall ist es unerlässlich, die Kommunikationswege zu reflektieren, will man die Kontroversen verstehen, die im Zeitalter des Imperialismus in Europa über Militäreinsätze in weit entfernten Weltregionen geführt wurden. Das Nebeneinander unterschiedlicher Kommunikationstechniken führte dazu, dass manche Nachrichten früher und andere später den Weg in die Zeitungen fanden. Der Kommunikationsweg hatte zudem Einfluss auf die Länge der übermittelten Botschaften und die auf diese Weise erfolgreich transportierten Deutungsmuster. Und die Hindernisse, die Nachrichten auf ihrem Weg überwinden mussten - von abgefangenen Läufern und gekappten Telegraphenleitungen bis hin zur Zensur der Militärbehörden - beeinflussten, was wann in welcher Länge in Europa veröffentlicht werden konnte und was keine Aussichten hatte, ans Licht der Öffentlichkeiten der kolonisierenden Staaten zu gelangen. In diesem Zusammenhang werden auch die Grenzen der Telegraphie deutlich, die im 19. Jahrhundert zu einem Wandel der globalen Kommunikation führte. Gerade wenn es darum ging, in Europa vorherrschende Deutungsmuster zu hinterfragen, waren Briefe und ihre Möglichkeit, sich ausführlich mitteilen zu können, weiterhin von großer Wichtigkeit.

\section{Literaturverzeichnis}

Arthur Abraham: Nyagua, the British, and the Hut Tax War, in: The International Journal of African Historical Studies 5 (1972), S. 94-98.

Arthur Abraham: Bai Bureh, the British, and the Hut Tax War, in: The International Journal of African Historical Studies 7 (1974), S. 99-106.

Stephen Badsey: War Correspondents in the Boer War, in: John Gooch (Hrsg.): The Boer War. Direction, Experience and Image, London/Portland 2000, S. 187-202.

Jacqueline Beaumont: The British Press During the South African War. The Sieges of Mafeking, Kimberley and Ladysmith, in: Mark Connelly, David Welch (Hrsg.): War and the Media. Reportage and Propaganda, 1900-2003, London/New York 2005, S. 1-18.

Brad Beaven: Visions of Empire. Patriotism, Popular Culture and the City, 1870-1939, Manchester u.a. 2012. 
Hugh Cecil: British Correspondents and the Sudan Campaign of 1896-98, in: Spiers (Hrsg.): Sudan. The Reconquest Reappraised, London/Portland 1998, S. 102-127.

Christopher Fyfe: A History of Sierra Leone, London u.a. 1962.

Daniel R. Headrick: The Tools of Empire. Technology and European Imperialism in the Nineteenth Century, New York/Oxford 1981.

Daniel R. Headrick: The Invisible Weapon. Telecommunications and International Politics, 1851-1945, Oxford/New York 1991.

John D. Hargreaves: The Establishment of the Sierra Leone Protectorate and the Insurrection of 1898, in: Cambridge Historical Journal 12 (1956), S. 56-80.

Thoralf Klein: Propaganda und Kritik. Die Rolle der Medien, in: Mechthild Leutner, Klaus Mühlhahn (Hrsg.): Kolonialkrieg in China. Die Niederschlagung der Boxerbewegung 1900-1901, Berlin 2007, S. 173-180.

Daniel R. Magaziner, Removing the Blinders and Adjusting the View: A Case Study from Early Colonial Sierra Leone, in: History in Africa 34 (2007), S. 169-188.

Christian Methfessel: »Oxident gegen Orient«. Europabilder in der Berliner Morgenpost während des Boxerkriegs, in: Themenportal Europäische Geschichte (2009), https:// www.europa.clio-online.de/essay/id/fdae-1513 (letzter Zugriff: 08.08.2019).

Christian Methfessel: Europa als Zivilisationsmacht. Kolonialkriege und imperialistische Interventionen in der deutschen und britischen Öffentlichkeit um 1900, in: Frank Bösch, Ariane Brill, Florian Greiner (Hrsg.): Europabilder im 20. Jahrhundert. Entstehung an der Peripherie, Göttingen 2012, S. 54-78.

Christian Methfessel: Kontroverse Gewalt. Die imperiale Expansion in der englischen und deutschen Presse vor dem Ersten Weltkrieg, Köln/Weimar 2019.

Jürgen Osterhammel: Die Verwandlung der Welt. Eine Geschichte des 19. Jahrhunderts, München 2009.

Kenneth Owen Morgan: The Boer War and the Media (1899-1902), in: 20th Century British History 13 (2002), S. 1-16.

Simon J. Potter: Jingoism, Public Opinion, and the New Imperialism, in: Media History 20 (2014), S. 34-50.

Tim Pratt: Ernest Jones' Mutinity. The People's Paper, English Popular Politics and the Rebellion 1857-58, in: Chandrika Kaul (Hrsg.): Media and the British Empire, Basingstoke/New York 2006, S. 88-103.

Ronald Robinson, John Gallagher, Alice Denny: Africa and the Victorians. The Climax of Imperialism in the Dark Continent, New York/London 1961.

Edward M. Spiers (Hrsg.): Sudan. The Reconquest Reappraised, London/Portland 1998.

Andreas Steinsieck: Ein imperialistischer Medienkrieg. Kriegsberichterstatter im Südafrikanischen Krieg (1899-1902), in: Ute Daniel (Hrsg.): Augenzeugen. Kriegsberichterstattung vom 18. zum 21. Jahrhundert, Göttingen 2006, S. 87-112.

Sally J. Taylor: The Great Outsiders. Northcliffe, Rothermere and the Daily Mail, London 1996.

Andrew Thompson: The Empire Strikes Back? The Impact of Imperialism on Britain from the Mid-Nineteenth Century, Harlow u.a. 2005.

Roland Wenzlhuemer: »Less Than No Time«. Zum Verhältnis von Telegrafie und Zeit, in: Geschichte und Gesellschaft 37 (2011), S. 592-613. 
Ute Wieland, Michael Kaschner: Die Reichstagsdebatten über den deutschen Kriegseinsatz in China: August Bebel und die »Hunnenbriefe«, in: Susanne Kuß, Bernd Martin (Hrsg.): Das Deutsche Reich und der Boxeraufstand, München 2002, S. 183-288.

Glenn R. Wilkinson: >There is No More Stirring Story«: The Press Depiction and Images of War During the Tibet Expedition 1903-1904, in: War \& Society 9 (1991), S. 1-16.

Merle Zeigerer: Kriegsberichterstatter in den deutschen Kolonialkriegen in Asien und Afrika. Augenzeugen, Anstifter, Komplizen?, Kiel 2016. 
Open-Access-Publikation im Sinne der CC-Lizenz BY-NC-ND 4.0 (C) 2021, Vandenhoeck \& Ruprecht GmbH \& Co. KG, Göttingen ISBN Print: 9783847112280 - ISBN E-Lib: 9783737012287 


\section{Stefan Geissler}

\section{Die Lloyd's List. Die Bedeutung des Postwesens für die frühneuzeitliche Marineversicherung}

\section{Einleitung}

"The masters of steamboats under contract with the Department, will deliver into the post offices (or to the local agent of the Department, if there be any), at the places at which they arrive, all letters received by them, or by any person employed on their boats, at any point along the route. ${ }^{1}$

Im Laufe des 19. Jahrhunderts wurde für alle Beteiligte im globalen Handelsverkehr ein relativ stabil funktionierendes Informationssystem immer wichtiger. Naturgemäß war man bemüht, das bestehende postalische Netzwerk für diese Zwecke einzusetzen, allerdings konnte das aufgrund der Zunahme des Welthandels nur ein erster Ausgangspunkt sein. Es stellte vielmehr das zugrundeliegende "Netzwerk der Netzwerke« dar, welches mit der etablierten (und mehr oder minder verlässlichen) Geschwindigkeit und vor allem Reichweite alle bestehenden und künftigen Netzwerke überspannte. ${ }^{2}$

Im Folgenden wird die Nutzung des Postwesens durch Händler und Marineversicherer vorgestellt, welche ihre Geschäfte im Laufe des 19. Jahrhunderts bei Lloyd's of London tätigten. Das besondere Augenmerk soll hierbei auf die aktive Einflussnahme der regulären postalischen Arbeitsabläufe gelegt werden, um Lloyd's einen Geschwindigkeits- und Wettbewerbsvorteil gegenüber der Konkurrenz zu ermöglichen. ${ }^{3}$

1 James T. Lloyd: Lloyd's Post Office Directory. Of the Unites States and Canadas, with new postal laws, New York 1863, S. 124. Bestandteil des Artikel 165, 03.03.1825.

2 Vgl. Wolfgang Behringer: Im Zeichen des Merkur. Reichspost und Kommunikationsrevolution in der frühen Neuzeit (Veröffentlichungen des Max-Planck-Instituts für Geschichte 189), Göttingen 2003, S. 685.

3 Wenn in dieser Arbeit von Lloyd's gesprochen wird, ist, wenn nicht anders dargestellt das steuernde und beschliessende Kommitee der Lloyd's of London gemeint. Allerdings hatten die gewählten Mitglieder auch immer ihre persönlichen Vorteile in ihrer Eigenschaft als Versicherer und Geldgeber im Blick. 
Die maritimen Händler der Frühen Neuzeit begegneten den diversen Gefahren der See und den damit verbundenen Risiken mit unterschiedlichen Maßnahmen. Maßgeblich hierbei war ein sehr gut funktionierendes Kommunikations- und Informationssystem, dass im Laufe der Jahrzehnte eine globale Ausdehnung erreichte. Durch den Aufbau (und Unterhalt) eines mit der Zeit global funktionierenden Versicherungswesens konnten vor allem die Eigner der Schiffe das Risiko eines Verlustes minimieren und die Gefährlichkeit einer Reise berechnen und damit abschätzen.

Wenn über Marineversicherungen in der (frühen) Neuzeit gesprochen wird, findet man sich sehr schnell bei Lloyd's of London wieder. ${ }^{4}$ Im frühneuzeitlichen London war es gewissermaßen Tradition, dass sich Gruppen mit gleichen (Handels-)Interessen in bestimmten Kaffeehäusern trafen, welche mit der Zeit dann synonym mit der entsprechenden Gesellschaft wurden. Zu den bereits existierenden Kaffeehäusern in London gesellte sich 1692 das namensgebende Lloyd's Coffee House, um fortan das geschäftliche Zentrum für all jene Personen zu werden, welche ein Interesse am Schiffsverkehr und maritimen Handel hatten. ${ }^{5}$ Der internationale Seehandel boomte ab dem 18. Jahrhundert und war dabei wesentlich günstiger und schneller als der Transport auf dem Landweg. ${ }^{6}$ Im Umfeld der Kaufleute, Reeder und Schiffshändler entwickelte sich aus einem Sicherheitsbedürfnis auf der einen, einer Gewinnorientierung auf der anderen Seite die Frühform eines maritimen Versicherungswesens, was den Grundstein für den heute noch existierende Versicherungskonzern Lloyd's of London darstellte.

Kaufleute streben seit Jahrhunderten nach Sicherheit und suchten daher nach Möglichkeiten, ihre Waren und deren Transport gegen unvorhergesehen Ereignisse abzusichern und das Risiko eines Ausfalls zu reduzieren. ${ }^{7}$ "[Merchants need] to know the world and its markets because knowledge was central

4 Im Folgenden wird oftmals die verkürzte Form Lloyd's verwendet, welche sich auch in den Quellen ab dem 19. Jahrhundert wiederfindet. Jedoch ist damit nicht die (Hamburger)-Lloyd oder Hapag-Lloyd gemeint.

5 Vgl. Ralph Davis: The rise of the English shipping industry. In the seventeenth and eighteenth centuries. 2. impr., Newton Abbot 1972, S. 163.

6 Vgl. Ronald Findlay, Kevin H. O’Rourke: Power and plenty. Trade, war, and the world economy in the second millennium (The Princeton economic history of the Western world), Princeton 2009, S. 382.

7 Anders als in einigen Arbeiten werden hier die Begriffe Risiko und Gefahr nicht Synonym verwendet.»Gefahren entsprechen unspezifischen Schadenspotentialen, wohingegen Risiken kalkulierbare Schadenspotentiale darstellen.« Vgl. Martin Lengwiler: Risikopolitik im Sozialstaat. Die schweizerische Unfallversicherung 1870-1970 (Industrielle Welt 69), Köln 2006, S. 37. 
to managing risk, whether it was the vessel itself, or markets as places or commodities. $\ll^{8}$

Ein Kaufmann in der frühen Neuzeit verfügte natürlich nur über eine bestimmte Menge an zu investierendem Kapital. Dieses wollte er möglichst gewinnbringend einsetzen und der Überseehandel versprach in dieser Zeit enorme Gewinne. Die Gefahr einer solchen Unternehmung war jedoch ebenso enorm. Bis zum 17. Jahrhundert lässt sich eine erhöhte Verlustrate im Seehandel feststellen, wenige Schiffe erreichten ein Dienstalter von mehr als 15 Jahren - bei einer einfachen Fahrtdauer von mehreren Monaten gen Asien eine sehr kostspielige Investition mit hohen Einsätzen. ${ }^{9}$ Viele Händler wagten dennoch das lukrative Geschäft, wollten die Gefahr eines Verlusts jedoch in gewissem Umfang beherrschen und in Form eines berechneten Risikos kalkulieren können. Kein Händler zu dieser Zeit wollte seine Schiffe und Waren als Hochrisikoanlagen betrachten, daher musste er sich intensiv mit den Themen Risikotragfähigkeit eines Marineversicherers und der eigenen Risikobereitschaft auseinandersetzen. Das Konzept des Risiko-Managements war im 18. Jahrhundert nicht unbekannt und wurde immer weiter verbessert. Mithilfe der Statistik und Mathematik konnten Daten in großem Umfang erfasst und ausgewertet werden. Gerade Lebens- und Marineversicherungen erlebten dadurch ihre erste Hochphase.

Die aufkommenden Versicherungen etablierten die institutionellen Grundlagen und damit die Sicherheit für einen Händler, seinen Handel oder Transport trotz eines bestehenden Risikos durchzuführen. ${ }^{10}$ Vor allem ab dem 18. Jahrhundert waren daher Londoner Kaufleute bereit, hochriskante Fernfahrten zu unternehmen, um mit den hierbei erzielten höheren Gewinnen den nahen Küsten- und Mittelmeerhandel zu überbieten. ${ }^{11}$ Mit der erhöhten Reichweite der Schiffe und ihrer langen Fahrtzeit wurden Informationen über ihren Standort und Zustand essentiell - sowohl für die Eigner wie auch die Versicherer.

8 Sheryllynne Haggerty: >Merely for money^? Business culture in the British Atlantic, 1750-1815 (Eighteenth-century worlds), Liverpool 2012, S. 55.

9 Vgl. Kenneth R. Andrews: Ships, money, and politics. Seafaring and naval enterprise in the reign of Charles I., Cambridge 1991, S. 32.

10 Vgl. Peter Borscheid: Systemwettbewerb, Institutionenexport und Homogenisierung. Der Internationalisierungsprozess der Versicherungswirtschaft im 19. Jahrhundert, in: Zeitschrift für Unternehmensgeschichte 51 (2006), S. 39.

11 Margrit Schulte Beerbühl: Deutsche Kaufleute in London. Welthandel und Einbürgerung (1660-1818) (Veröffentlichungen des Deutschen Historischen Instituts London 61), München 2007, S. 328. 
»With the practice of insuring ships and their cargoes against sea risk there would naturally arise the necessity of adopting means to ascertain whether the vessels were seaworthy, and to have the relative qualities of ships in this respect classified and recorded in some manner convenient to persons interested in shipping. " ${ }^{12}$

Eine Antwort auf die zunehmenden Bedürfnisse der (britischen) Kaufleute war die Lloyd's List mit ihren späteren Erweiterungen. Diese hochspezialisierte Wirtschaftszeitung, zu Beginn des 18. Jahrhunderts durch Edward Lloyd im Umfeld des Lloyd's Coffee House entstanden, dient noch heute als Aggregator dieser Informationen und Wissen für Schifffahrtsindustrie und Handel. ${ }^{13} \mathrm{Im}$ namensgebenden Londoner Kaffeehaus trafen sich seit 1691 Geschäftsleute und private Versicherungsgeber, um über Risikoversicherungen von globalen Schiffstransporten zu verhandeln. Die einige Jahre später gegründete Society of Lloyd's (die spätere Lloyd's of London) institutionalisierte diese Verbindungen und stellte den Beginn einer historisch bedeutenden Versicherungsgesellschaft dar. ${ }^{14}$ Bis in das 19. Jahrhundert hinein entwickelte sich London so zum "globalen Zentrum der maritimen Versicherungen $« .{ }^{15}$ Genaue Informationen über Lage, Route und Beladung von Schiffen hatten dabei einen sehr hohen Stellenwert. Diese wurden in den Lloyd's Lists gesammelt und zusammen mit anderen die Schifffahrt betreffenden Meldungen und Werbeanzeigen wöchentlich herausgegeben.

Der Inhalt dieser Zeitung veränderte sich je nach den Bedürfnissen der Leser, stellte jedoch nur eine Zusammenfassung der gesammelten Informationen bei Lloyd's dar. Im konkreten Versicherungsfall wurden natürlich alle Informationen zusammengetragen, um allen Beteiligten die bestmögliche Unterstützung zu gewähren. ${ }^{16}$

12 Lloyd's Register of Shipping: Annals of Lloyd's Register. Being a sketch of the origin, constitution, and progress of Lloyd's Register of British \& foreign shipping, London 1884, S. 3.

13 Auch wenn diese beiden Begriffe ohne Unterscheidung verwendet werden, sind sie für dieses Thema klar zu unterschieden. Ich verwende hierfür unter anderem die Arbeiten von Peter Burke (Peter Burke: Die Explosion des Wissens. Von der Encyclopédie bis Wikipedia, Berlin 2014.) und Ikujiro Nonaka (Ikujiro Nonaka: A Dynamic Theory of Organizational Knowledge Creation, in: Organization Science 5/1 (1994), S. 14-37, hier S. 15: »In short, information is a flow of messages, while knowledge is created and organized by the very flow of information, anchored on the commitment and beliefs of its holder."

14 Anders als im Bereich der Feuerversicherungen als zweitem Pfeiler der privaten Versicherungen existiert bisher keine quellennahe Studie über diesen Teil der (europäischen) maritimen Versicherungsgeschichte. Vgl. Cornel Zwierlein: Der gezähmte Prometheus. Feuer und Sicherheit zwischen Früher Neuzeit und Moderne, Göttingen 2011.

15 Peter Borscheid: Europe. Overview, in: Ders. Niels Haueter: World Insurance. The evolution of a global risk network, Oxford 2012, S. 39.

16 Wie drastisch diese Reduzierung der Information sein konnte, zeigt sich bei einem Blick in die jährlichen »Reports of Wrecks and Casualties« der Lloyd's. Hier wurden im Falle eines beschädigten oder verlorenen Schiffes alle Informationen zusammengetragen, aber auch die Exzerpte 
$\mathrm{Zu}$ Beginn enthielten die später sogar täglich erscheinenden Ausgaben der Lloyd's List Informationen über den globalen Schiffsverkehr. Wie beschrieben deckten die Inhalte sehr unterschiedliche Bereiche ab, gemeinsam war ihnen jedoch die inhaltliche Ausrichtung auf den (maritimen) Geschäftsmann seiner Zeit.

\section{Das frühneuzeitliche maritime Versicherungswesen}

Marineversicherungen existierten bereits seit Jahrhunderten, so lässt sich für London eine überlieferte Police aus dem Jahr 1426 nachweisen. Lombardische und venezianische Händler entwickelten im Laufe der Zeit einen Weg, die Effekte der allgegenwärtigen Bedrohungen der See zu senken. ${ }^{17}$ Die Kosten zur Behebung von Schäden oder gar die komplette Erstattung wurden so weit wie möglich verteilt, um die Belastung des einzelnen $\mathrm{zu}$ minimieren. Durch die Zunahme des verfügbaren Kapitals eines Kaufmanns wurde daraus ein Geschäft, um Sicherheiten gegen mögliche Risiken zu bieten.

»Risks consequently have to be understood as permanent companions of everyday life. As long as people value certain things or conditions and as long as they take decisions in the presence of uncertainty, they will face risks. Risks are hence a basic constituent of life. « ${ }^{18}$

Der Umgang mit Risiken gehörte zum alltäglichen Geschäft eines frühneuzeitlichen Händlers. Wenn er sie als zu bedrohlich betrachtete konnte die Sicherheit einer Versicherung verlockend sein. Heruntergebrochen war ein Warentransport über die Ozeane nunmehr eine mehr oder minder komplizierte mathematische Gleichung. Risiken und Gefahrenpotentiale wurden mit Versicherungen und $\mathrm{zu}$ erwartenden Gewinnen verrechnet, und wenn das Ergebnis nach Wunsch des Kaufmanns war, wurde der Transport angeordnet. Das Wissen um Risiken, samt deren Berechnung aufgrund von Statistiken und anderer Daten, war für diese Gleichungen elementar und wurde durch Lloyd's und mit der Lloyd's List zur Verfügung gestellt. Dies ist für ein Versicherungsunternehmen nicht üblich, da solche Daten und Informationen für die eigenen Mitarbeiter natürlich mehr als nützlich waren und sind, für mögliche Kunden jedoch einen zu genauen Einblick in die eigene Arbeit gewähren. Gleichzeitig

für die Ausgabe der Lloyd's List definiert. Aus einem zwei bis dreiseitigen Eintrag konnten dann schnell nur ein oder zwei Sätze übrigbleiben. Eine drastische Reduktion der übermittelten Information für die Leserschaft.

17 Vgl. Adrian Leonard: Gresham and Defoe (Underwriters). The Origins of London Marine Insurance, London 2014, S. $2 \mathrm{f}$.

18 Terje Aven, Ortwin Renn: Risk Management and Governance. Concepts, Guidelines and Applications, Heidelberg 2010. 
ist es für die Wirtschaftlichkeit eines Unternehmens wünschenswert, diese Daten nicht nur einem Kunden beziehungsweise einer Nutzungsart zur Verfügung zu stellen. Lloyd's begann daher, individuell zugeschnittene Informationspakete für Kunden bereitzustellen, um deren Bedürfnisse zu befriedigen. ${ }^{19}$

Die Weitergabe dieser Daten erfolgte im Laufe des 18. Jahrhunderts allerdings nur sehr langsam. Die aktuellsten Neuigkeiten eines ausländischen Hafens konnten häufig Monate alt sein. Ebenso konnten aber natürlich Schiffe ohne eine Spur verloren gehen. Dieser Umstand machten diese wenigen und nach unserem heutigen Verständnis alten Informationen wiederum wertvoll. Für einen Versicherer war jede Information über den Zustand und Verbleib eines Schiffes und/oder seiner Waren von enormer Wichtigkeit, bedeutete es doch im Ernstfall, ob er für einen Versicherungsfall aufkommen musste oder nicht. Auch konnte es sich als sinnvoll erweisen, von mehr oder minder unbeteiligter Seite Nachricht über den Verbleib eines Schiffes zu erhalten - allein um etwas mehr vor böswilligen Betrugsfällen geschützt zu sein.

Die Informationen erreichten Lloyd's zeitgemäß über den Postweg, zum Ende des Jahrhunderts wurde zudem das aufkommende Semaphoren-, später auch das Telegraphennetzwerk genutzt. Für eine bessere Einordnung wenden wir uns kurz dem Postwesen der Frühen Neuzeit zu, im speziellen jenen Bereichen, welche für die Vorgänge bei Lloyd's von Interesse waren. Daran anschließend werden die verschiedenen Stationen aufgezeigt, wie Lloyd's Informationen sammelte und welche auch teilweise heute noch im Einsatz sind.

\section{Die Nutzung der (britischen) Post durch Lloyd's}

Die Ausbildung des frühneuzeitlichen Postwesens vor allem in Europa kann hier nur partiell besprochen werden, für tiefere Ausführungen sei auf die entsprechende Fachliteratur verwiesen. ${ }^{20}$

Während der ersten Jahre bediente sich die sich langsam bildende Gemeinschaft innerhalb des Kaffeehauses noch privater Informationen einzelner Kaufleute, die sie bereitwillig mit ihren Kollegen und Freunden teilten. Dies gehörte

$19 \mathrm{Vgl}$. Mark Andrejevic: Infoglut. How too much information is changing the way we think and know, New York 2013, S. 60.

20 Exemplarisch seien hier die Arbeiten von Behringer (Wolfgang Behringer: Im Zeichen des Merkur. Reichspost und Kommunikationsrevolution in der frühen Neuzeit. (Veröffentlichungen des Max-Planck-Instituts für Geschichte, 189) Göttingen 2003; Ders. Thurn und Taxis. Die Geschichte ihrer Post und ihrer Unternehmen, München 1990), van den Heuvel (Gerd van den Heuvel: Leibniz im Netz. Die frühneuzeitliche Post als Kommunikationsmedium der Gelehrtenrepublik um 1700, Hameln 2009) und Arndt\&Körber (Johannes Arndt, Esther-Beate Körber (Hrsg.): Das Mediensystem im Alten Reich der Frühen Neuzeit 1600-1750, Göttingen 2010). 
auch noch später zum guten Ton. ${ }^{21}$ So finden sich in den Komitee-Berichten ab 1780 einzelne Vergütungen für diverse Ankunfts- und Abfahrtslisten einzelner (Übersee-)Häfen:

»17.02.1781: Gave a Draft to Thomas Taylor for 43 Pound 14 Pences (Sound Convoy List $5 £ 5$; West India L. $5 £ 5$.

1783 - Sound List Subscriptions: Baltic Lists $5 £ 5$; Mr. Macau[n]ley two years Sound Lists $30 £ \Perp^{22}$

Diese Listen wurden entweder direkt von den Kaufleuten zu Lloyd's gebracht, oder aber über den privaten Briefverkehr versendet. Eine solche postalische Kommunikation fand jedoch $\mathrm{zu}$ einer Zeit der großen Unsicherheit sowie langsamem und unregelmäßigen Kommunikationswegen statt. Die Reisedauer von Briefen betrugen selbst zur Mitte des 19. Jahrhunderts bei den besten Verbindungen von New York nach London über einen Monat, und selbst von Lissabon immer noch eine knappe Woche. ${ }^{23}$ Eine regelmäßige zeitnahe Informationsübermittlung war daher nicht möglich. Einzig für den Bereich der britischen Inseln konnte hier eine gewisse Aktualität gewährleistet werden. ${ }^{24}$ Für die Kaufleute und Underwriter genügte jedoch in den Anfangsjahren das Wissen, ob ein Schiff wohlbehalten am Zielhafen angekommen war, oder im Ernstfall ein Versicherungsfall vorlag, welcher dann Untersuchungen und Zahlungen nach sich zog (siehe Abbildung 1). ${ }^{25}$

Das Verlangen nach mehr Informationen in kürzeren Abständen wuchs beständig mit dem Wachstum des (britischen) Seehandels. Schon bald genügten die mehr oder minder zuverlässigen Zuträger nicht mehr, und das Komitee der Lloyd's of London stellte in den wichtigsten britischen und irischen Hafenstädten sowie später auch in den Überseegebieten ständige Korrespondenten ein, welche in ihren Heimatorten Informationen sammelten und diese mehrmals

21 Vgl. Richard Chamberlain, The History Lloyd's Intelligence Unit, unpublished typescript 1942, Guildhall Library, SL 64:9, S. 1.

22 Minutes of the Committee of Lloyd's, Dec 1771-Aug 1804, Guildhall Library [GL], MS31571001, S. 71 und S. 75. Die angegebenen Summen in Pfund Sterling und Schilling resultieren aus der damaligen Umrechnung: 1 (Gold-) Guinea $=1$ Pfund Sterling und 1 Schilling. Die Erstellung \& Transport der allermeisten Listen wurde demnach mit 5 Guineas im Jahr entlohnt. Ein Vergleich der Kaufkraft gegenüber heutigen Werten ist wie immer ein schwieriges Unterfangen. Wenn man allein den Goldpreis zu Rate zieht, kann man einen Guinea mit grob 300 Euro aufrechnen. Die Listen waren in jedem Fall eine lucrative (Neben-) Erwerbsquelle.

23 Vgl. Hierzu ausführlich die Übersichtstabellen bei Lloyd. Lloyd, Post, S.120ff.

24 Vgl. Alan Cameron, Roy Farndon: Scenes from sea and city. Lloyd's List 1734-1984, London 1984, S. 259.

25 Die Arbeitsweisen der Underwriter, Subscriber und die Informationsnutzung innerhalb Lloyd's wird später ausführlicher beschrieben. 
wöchentlich auf dem Postweg in Form von Abfahrts-, Ankunftslisten und Verlustübersichten in die Lombard Street zu Lloyd's schickten:

»Read a Letter from Mr. Richard Cock Junior at Barbadoes, dated 12th December, acknowledging the receipt of a Letter ordered to be written him on 27th October transmitting a List of Arrivals, and stating that he shall endeavor to forward it on a more copious plan, and hopes it will prove satisfactory to the Committee. $\aleph^{26}$

In einem weiteren Bestreben, zusätzliche Informationen zu erhalten, bediente sich Lloyd's bei Konkurrenzunternehmen. So wurden bereits früh Verträge mit der Liverpool Underwriter's Association geschlossen, um eine regelmäßige Lieferung mit Informationen zu gewährleisten. ${ }^{27}$ Diese Lieferung war jedoch offenbar nicht einseitig. Bereits früh bemühten sich die Underwriter aus Liverpool um Zugang zu den exklusiven Informationen, welche Lloyd's durch Kontakte zur britischen Admiralität erlangte:

"You will not (it is to be hoped) refuse them the Lists for transcription, when you have made the same use of them, and particularly when they readily agree to pay a proportion of the expense of procuring them from the Admiralty (the Lists to be reguarly returnd to Mr. B). $\ll^{28}$

Nach längeren Verhandlungen wurde eine Zahlung von 200 Pfund/Jahr vereinbart, was später wie aufgezeigt zu weiteren Informationshandeln führte.

Alle Briefsendungen trafen nun mit der jeweils geltenden Häufigkeit in den neuen Räumlichkeiten in der Lombard Street ein, in welche Lloyd's zum Ende des 18. Jahrhunderts das New Lloyd's Coffee House verlegte. Dies geschah nicht "zufällig - im Nachbargebäude befand sich das General Post Office. ${ }^{29}$ Die Anstellung der Korrespondenten zeigt, dass Lloyd's eine erste Professionalisierungsphase startete, um die Sammlung und Verarbeitung von Informationen zu verbessern. Dies kulminierte später in der ständigen Überarbeitung und Vergrößerung der Lloyd's List, was weiter unten behandelt wird. Wenden wir uns zunächst jedoch wieder der besonderen Nähe zum General Post Office und damit dem Post Master General in London zu. Mit dem Beginn des 17. Jahr-

26 Bericht vom 02.02.1807, in: Minutes of the Committee of Lloyd's, Sep 1804-Dec 1809, GL, MS31571-002, S. 180. Weitere Einträge finden sich auch im vorigen Jahrband, ab 1805 beginnt jedoch eine stetigere Kommunikation zwischen den Korrespondenten und dem Komitee, um die Spezifika der Informationsübermittlung genauer zu bestimmten.

27 Die verschiedenen Vertragsversionen und auch jene mit anderen Zulieferern sind in einem gesonderten Archivband aufgelistet: Lloyd's of London: Contracts for Supplies of Intelligence, Guildhall Library, MS31648. Zudem finden sich in vielen Berichten des Komitees immer wieder Verweise auf die bestehende Abmachung, vgl. hierzu Chamberlain, Intelligence, S. 9 und S. $136 f$.

28 Minutes of the Committee of Lloyd's, Jan 1810 - Aug 1811, GL, MS31571-003, S. 60.

29 Sehr anschaulich in einem Kupferstich von circa $1793 \mathrm{zu}$ sehen. Ein Abdruck findet sich unter anderem bei Cameron, Scenes, S. 15. 
hunderts wurde nach und nach in ganz Europa das Postwesen institutionalisiert, allerdings wurden die wenigsten Post-Bediensteten nur hinreichend entlohnt, wenn überhaupt. Es bestand folglich ein großes Interesse, zusätzliche Einnahmequellen zu erschließen. Eine Möglichkeit war, zusätzlich zu den normalen Zustellungen, private Kurierdienste zu übernehmen oder auch zusätzliche Positionen in anderen Arbeitsgebieten zu übernehmen, welche sich mit der Hauptarbeit im Postwesen vereinbaren ließen. ${ }^{30}$

Für eine so hohe Position wie jene des Post Master General fand sich in Lloyd's ein idealer Handelspartner. Die privaten Portokosten für sämtliche zu sendenden Informationspakete und Briefe stiegen immer weiter. Bis zum Jahre 1791 wurde daher vereinbart, dass Lloyd's of London dem Post Master General jährlich 200 Pfund sandte. ${ }^{31}$ Im Gegenzug konnten alle Underwriter und Korrespondenten weltweit portofrei ihre Listen und sonstigen Berichte über den Schiffsverkehr nach London schicken:

$»[\ldots]$ it having been the custom for many years to permit Ship News to pass free of Postage, the Committee have lately had forms printed and sent to several of the Agents in order that only Shipping Intelligence may be inserted, and by the enclosed circular to the Agent you will also learn that they are particularly directed in what manner they should address their Letters and Lists. ${ }^{32}$

Es genügte, als Empfänger »Lloyd's« anzugeben - die Post erledigte den Rest. Dies war eine gängige Praxis durch das gesamte 19. Jahrhundert hindurch. ${ }^{33}$ Später gab es (zusätzlich) noch ein jährliches Geschenk an den Sekretär des General Post Office. So erhielt etwa Mr. Freeling ab 1824 immer zu Weihnachten eine 70 Pfund schwere Schildkröte, "as an acknowledgment on the part of the Committee of the accommodation afforded by the Department under his superintendence. ${ }^{34}$ Diese besondere Zuwendung lässt sich mindestens bis 1836 in den Berichten nachweisen.

Bevor wir uns nun den Agents und damit der zweiten Professionalisierungsstufe zuwenden, müssen wir zunächst den Handelsplatz Lloyd's of London und die dort tätigen Kaufleuten vorgestellt bekommen, um dieses besondere Konstrukt besser zu verstehen.

30 Vgl. Heiko Droste: The Terms of Royal Service. Post Servants' Finances, c. 1700, in: Heiko Droste (Hrsg.): Connecting the Baltic Area. The Swedish Postal System in the Seventeenth Century, Huddinge 2011, S. 1-5.

31 Vgl. unter anderem Chamberlain, Intelligence, S. 1 und Cameron, Scenes, S. 15.

32 Minutes of the Committee of Lloyd's, Sep 1824-Feb 1826, GL, MS31571-008, S. 6.

33 Vgl. Cameron, Scenes, S. 6.

34 Minutes of the Committee of Lloyd's, Sep 1823-Feb 1824, GL, MS31571-007, S. 348. 


\section{Lloyd's of London im 19. Jahrhundert}

Lloyd's of London belegt auch heute noch einen oberen Platz innerhalb der maritimen Versicherer, versteht sich selbst allerdings nicht als ein Versicherungsunternehmen im engeren Sinn, sondern als ein »international einzigartiger Versicherungsmarkt « ${ }^{35}$. Lloyd’s stellte seit jeher lediglich ihre Räumlichkeiten und andere Dienstleistungen für zahlende Mitglieder zur Verfügung, und erhielt bei erfolgreichem Geschäftsabschluss eine Provision. Neben dem geschäftlichen Umfeld verstanden es die Verantwortlichen, ihren Marktplatz mit weiteren Assets zu versehen, um die Underwriter bei ihren Geschäften zu unterstützen und um sie auch weiterhin als Mitglieder zu halten. ${ }^{36}$

Ein Underwriter war ein privater Kapitalgeber, welcher durch einen Broker für eine mögliche Versicherung ins Spiel gebracht wurde. Wenn er das Risiko eingehen wollte, unterschrieb er die Versicherungs-Police mit seiner gewünschten Summe. Wenn kein Zwischenfall eintrat, konnte er die gewonnene Gebühr einstreichen und hatte damit sein Geld gut angelegt. ${ }^{37} \mathrm{Um}$ im Falle einer Beschädigung oder eines Totalverlustes nicht einen zu großen Verlust zu erleiden, teilten sich viele Underwriter das Risiko einer Police. Nur wenige hatten jedoch die Verbindungen und die Möglichkeiten, sich umfassend mit allen Informationen, den Risiken und den beteiligten Personen zu befassen. Nur ganz erfahrene Underwriter (meist ehemalige Kaufleute im Ruhestand) wagten sich an die Versicherung von sogenannten cross risks, also den Fahrten zwischen zwei ausländischen Häfen. Nach einer Weile etablierte sich daher das System des lead underwriters. Er zeichnete als erstes eine Versicherungs-Police, bestimmte meist den zu zeichnenden Betrag für jeden weiteren Underwriter und übernahm zudem die Kommunikation zwischen dem Broker, dem Versicherten und Lloyd's. ${ }^{38}$

Die bereits angedeuteten Professionalisierungsphasen hoben Lloyd's in den Bereichen Risikomanagement und Bewertung weit über mögliche Mitbewerber, so dass ein Großteil des britischen Handelsvolumens im 19. Jahrhundert (und darüber hinaus) bei Lloyd's versichert wurde. Bereits früh zeigte sich diese Marktposition in den Selbstevaluationen und den Marktanalysen:

35 Vgl. Maria Kyriakou: From discreteness to cooperation. Relational contracting in the London marine insurance market [Dissertation], London 2002, S.158.

36 Vgl. für eine moderne Definition der Underwriter: Kyriakou, discreteness, S. 165.

37 Weiter Erklärungen zu den Underwritern und Brokern finden sich bei Kingston: Christopher Kingston: Adverse Selection and Institutional Change in Eighteenth Century Marine Insurance, Edinburgh 2008, S. 2ff.

38 Vgl. Christopher Kingston: Marine Insurance in Britain and America, 1720-1844. A Comparative Institutional Analysis, in: The Journal of Economic History 67 (2007), S. 386. 
»That the arrangements at Lloyd's have so perfected the system of Insurance business, as to have given to the Subscribers the most efficient and respectable correspondents in every considerable maritime town in the civilized world, whereby a course of information is secured, beneficial in a vast variety of modes, not only to the Underwriters, but to the public. This system has grown by slow degrees, is the result of long and costly experience, and once dispered, would require ages to renew and re-establish. « ${ }^{39}$

Dieses System hatte verschiedene historisch gewachsene Elemente, ein zentrales stellte die Lloyd's List dar.

\section{Die Entstehung der Lloyd's List}

Sie entstand durch systematisches Sammeln, Kompilieren und Herausgeben von Abfahrts- und Ankunftsnachrichten von Schiffen aus englischen und kontinentalen Häfen (siehe Abbildung 2):

»On the front page appeared the rates of exchange, the microscopic Stock Exchange List of the time, and a few other items of general business interest. The back page was given over to the `Marine List $<$ - arrivals, and occasionally sailings, reported from the principal English and Irish ports [...] a few paragraphs relating to casualties, speakings, missing vessels, and other items of interest to merchants, shipowners, and underwriters. « ${ }^{40}$

Immer wieder an die Bedürfnisse der Underwriter angepasst, kann man ab dem späten 18. Jahrhundert von einer ersten Professionalisierung der Zeitung sprechen. Um 1796 entschloss sich das Committee of Lloyd's zum Einsatz eines Secretary mit besonderen Aufgabenbereichen:

»The Committee taking into their serious consideration the Arrangement necessary to be made at Lloyds [...] and that the nature and extent of the Business of the Subscribers of Lloyds requires that there should be a Secretary [...] and to whom through the Master all Articles of Intelligence shall be conveyd and under whose Authority subject to the control of the Committee all information should be regulated for Publication and that in the frequent occasions of intercourse between Lloyds and the different Offices under Government such an Establishment would have peculiar advantages $[\ldots]$ «1 $^{41}$

In den Grundzügen ist hier bereits das spätere Informationsnetzwerk von Lloyd's vorgestellt. Der Secretary, später das entsprechende Sub-Committee of Intelligence, überwachte den Eingang aller Intelligence und Informationsbruchstücke, um dann im besten Interesse seiner Subscriber zu entscheiden, was damit passieren sollte. Die Kontrolle des Zugangs zu diesen Informationen

39 Report of the Special Committee 1824, GL, 8244, S. $35 f$.

40 Lloyd's List \& Shipping Gazette. 1734-1934, 200 years of shipping news, GL, Fo Pamphlet 288, S. 7.

41 Minutes, MS31571-001, S. 124. 
verdient besondere Beachtung, da dies einen entscheidenden Vorteil gegenüber möglichen Konkurrenten darstellte. Welche Informationen nun in London eintrafen, wie dieses System aufgebaut wurde und wer letztlich darauf Zugriff hatte, soll im Folgenden beschrieben werden.

\section{Information als Ware}

Verursacht durch verschiedene Brände innerhalb Londons und speziell in den Gebäuden von Lloyd's beinhaltet der überlieferte Quellenbestand keinerlei Akten vor 1760. Dennoch lassen sich aus anderen Beständen die Anfänge der maritimen Informationsübermittlung in den Sound- und Marine-Lists identifizieren. Ein Sound bezeichnet den Zustand eines Schiffes und gibt Aussage über seine Einsatzfähigkeit, was für jeden Händler eine entscheidende Information darstellte. Auf einem fahruntüchtigen Schiff wollte niemand seine Waren transportieren lassen. ${ }^{42}$ Lloyd's erwarb alle verfügbaren Sound-Lists, um den Underwritern eine Auswahl verlässlicher Schiffe zu ermöglichen: »That it be recommended to the Committee to procure Lists of all Convoys together with the Sound[s] Lists, and that whatever Expences attend procuring the same $[\ldots] \ll^{43}$. Diese Nachrichten stellten nicht nur eine abstrakte Informationssammlung dar, sie wurden zu einem materiellen Produkt, dass man kaufen, verkaufen - und somit handeln konnte. ${ }^{44}$

Das Sammeln der Zustände von Ozean-, oder Küstenschiffen wurde bereits früh institutionalisiert. Lloyd's erstellte das Lloyd's Register, dessen fragmentarisch überlieferte Versionen auf 1764 und 1786 zurückdatieren. Spätere statistische Daten lieferten den Kaufleuten einen sehr genauen Überblick über den jeweils aktuellen Stand der britischen Handelsmarine und jener ausländischen Schiffe, die regelmäßig mit ihnen Geschäfte machten. ${ }^{45}$ Der Zweite Bestandteil aus den Anfangstagen waren die Marine Lists, eine Auflistung von Abfahrten und Ankünften der Handelsschiffe, welche durch Hafenregistraturen, Subscriber oder Freunde in den entsprechenden Häfen gesammelt und nach London verschickt wurden. Um den Wert dieser Informationen für einen Subscriber

42 Siehe für eine ausführliche, zeitgenössische Definition: Manuel Llorca-Jaña: The Marine Insurance Market for British Textile Exports to the River Plate and Chile. 1810-50, in: The development of international insurance, in: Robin Pearson (Hrsg.), London 2010, S. 25-36, hier S. 32. 43 Minutes, MS31571-001, S. 66.

44 Vgl. Robertson, Frances: Print Culture. From Steam Press to Ebook (Directions in Cultural History), London 2013, S. 54.

45 Für eine nach Jahreszahlen aufgeschlüsselte Inhaltsübersicht des Lloyd's Register siehe: Centre for Port \& Maritime History: Infosheet 52, Lloyd's Marine Insurance Records, Merseyside Maritime Museum, Liverpool 2004, S. 2. 
bei Lloyd's einschätzen zu können, muss man sich dessen zwei mögliche Rollen verdeutlichen. Zunächst musste er einer der erlaubten Professionen nachgehen: „Only Merchants, Bankers, Underwriters, and Insurance Brokers, shall be permitted to become Subscribers on being recommended to the Committee ${ }^{46}$. Der Zugang zu Lloyd's war also absichtlich beschränkt, und dies traf nicht nur auf den Beruf zu (siehe Abbildung 3). So mussten sich Subscriber für oder gegen Lloyd's entscheiden, eine parallele Mitgliedschaft bei Lloyd's und einem anderen maritimen Versicherer war nicht gestattet. Dadurch konnten sie die erste mögliche Funktion eines Subscribers bei Lloyd's wahrnehmen und als Händler direkt von den bereitgestellten Informationen profitieren. Weiterhin waren permanent Bedienstete in den Räumlichkeiten anwesend, welche den Zugang der Besucher kontrollierten. So musste jeder Subscriber oder dessen temporäre Vertretung eine Elfenbein-Marke vorzeigen, um $\mathrm{Zu}$ gang in die inneren Räume von Lloyd's zu erhalten:

$» 5^{\text {th }}$ That a Door shall be open' $d$ in the second or inner Room under the Clock by which Door only, all Subscribers their Substitutes, or Clerks shall enter, upon shewing their Tickets to the Door Keeper. The Door to open inwards, and no Person to be permitted to go out at that Door.

$6^{\text {th }}$ That the present (or outer) Door shall be the entrance into the Lobby, which shall be appropriated for the use of Non_Subscribers who may have Business to transact with Subscribers."

Lloyd's etablierte also eine sehr starke Abgrenzung nach außen, was diese Institution noch interessanter für vermögende Kaufleute machte. Neue Handelspartner hatten es sehr schwer, denn die bestehenden Subscriber vermieden es generell, Geschäfte mit Personen zu tätigen, zu denen sie keinerlei Verbindung hatten. ${ }^{47} \mathrm{Ab} 1800$ wurde festgelegte, dass jeder neue Subscriber (und später jeder Agent) durch mindestens zwei Subscriber vorgeschlagen und anschließend nach einer Wahl durch das Committee of Lloyd's bestätigt werden musste:

»Resolved, that in future any Recommendation of a new Subscriber to these Rooms, being shown to two of the Committee, it shall be sufficient should they approve of the Gentleman recommended; if not it must remain till the Committee shall meet. ${ }^{48}$

Die Welt des Handels war eine vernetzte Welt, und da die Komplexität immer mehr zunahm, benötigte selbst ein erfahrener Kaufmann die Hilfe von Experten, welche ein starkes, weltweit operierendes Informationsnetzwerk bereitstellen konnten. Lloyd's of London erkannte diese Bedürfnisse und etablierte ein

46 Minutes, MS31571-001, S. 196.

47 Vgl. Kingston, Adverse Selection, S. 15.

48 Minutes, MS31571-001, S. 218. 
umfangreiches System, um diese Informationen gegen eine Gebühr bereitzustellen. Dieses System war die Lloyd's List. Die Weltmeere waren ein Transportweg voller Unsicherheiten, und die Lloyd's List brachte gegen Geld ein gewisses Grad an Sicherheit. Sie bedienten schon in dieser Zeit ein Bedürfnis, das uns allen heute bekannt ist: Die Unwissenheit und Unsicherheit durch Wissen und detailgenaue Informationen zu beenden. »[...] A sprawling array of public and private agencies was collecting more information than anyone could possibly comprehend. « ${ }^{49}$ Für den Grossteil der Menschen war die bereitgestellte Informationsfülle tatsächlich nicht zu bewältigen, für einige war sie jedoch im wortgetreuen Sinne Gold wert.

Wenn nun ein britischer Kaufmann ein Subscriber bei Lloyd's war, konnte er mit diesen Informationen seine Handelstätigkeit signifikant verbessern. Zunächst waren ihm durch die beigefügten Preis-Indexe der einzelnen Häfen bestimmte Angebots- und Nachfragesituationen bekannt, auf die er reagieren konnte. Wenn er ein Schiff zum Transport gebucht hatte, beschrieben aufeinander folgende Ausgaben der Lloyd's Lists den Reiseweg dieses Schiffes, wenn sie überwachte Häfen ansteuerten. Auch wenn die Nachrichten von den Häfen nach London natürlich Tage oder Wochen brauchten, waren sie dennoch signifikant schneller als das überwachte Schiff. ${ }^{50}$ Ein Schiff war ein sehr teures Transportmittel und seine Besitzer wollten das Risiko eines Verlusts so weit wie möglich minimieren. Schiffseigner und Kaufleute suchten die detailreichsten Informationen über die Position des Schiffes, um in den Zielhäfen rechtzeitig die jeweiligen Verkäufe vorzubereiten.

Bei einem Warentransport über sehr lange Distanz war ein genaues Timing erforderlich, damit das Unternehmen Gewinn abwarf. Die punktgenaue Ankunftszeit eines Schiffes mit dringend benötigten Waren konnte zwischen Reichtum und Bankrott entscheiden. Man kann hier sicherlich das bekannte Sprichwort »Zeit ist Geld « verwenden. Je nach Zielort konnte der Händler mit Hilfe entsprechender Informationen noch rechtzeitig reagieren und entsprechende Vorbereitungen treffen, um seine Waren möglichst erfolgreich zu verkaufen. Bei längeren Fahrten, beispielsweise nach Asien oder Amerika, war eine Benachrichtigung in Verbindung mit Anweisungen des Händlers im Zielhafen zeitlich kaum möglich, auch wenn die schnellen Postschiffe ihre Handelspendants weit hinter sich ließen. Dennoch waren diese Informationen

49 Andrejevic, Infoglut, S. 11.

50 Die sogenannten Packet Line Ships hatten natürlich auch mit den Wetterumständen zu kämpfen, verkürzten jedoch generell durch ihre leichtere und auf Schnelligkeit ausgelegte Bauweise die Reisedauer im Vergleich zu den Handelsschiffen der Kaufleute. Vgl. weiter: Jessica Karagöl: Girdling the Globe, networking the world. A discourse analysis of the media representation of nineteenth-century transport and communication technologies in Victorian Britain, 1838-1871 [Dissertation], Heidelberg 2013, S. 51f. 
nicht wertlos, so konnte ein Händler sich zumindest sicher sein, dass seine Waren unbeschadet bei seinen Partnern angekommen waren, und erfuhr dies nicht erst nach Monaten des Wartens und Bangens. Der Händler erfuhr so natürlich auch rechtzeitig über eine Beschädigung, deren Reparatur die Fahrt erheblich verlängerte oder im schlimmsten Falle unmöglich machte. Wenn man einen Handel über die Weltmeere betrieb, nahm man das Risiko des Scheiterns in Kauf, denn "carriage by sea was, however, liable to interruption and delay «. ${ }^{51}$ Hier kommt die zweite Rolle eines Subscribers bei Lloyd's ins Spiel, die des Underwriters.

»Because interpreting all this information to determine premiums involved weighing numerous factors, underwriting was ultimately a matter of individual judgment, requiring experience and familiarity with the routes, vessels, people, and circumstances involved. Lloyd's attracted a wide variety of underwriters, many of whom were active or retired merchants, and whose specialist knowledge of particular kinds of risks enabled them to make these kind of judgments. « ${ }^{52}$

Ein Underwriter versicherte mit seinem Privatvermögen Schiffe oder Ladung gegen Beschädigung oder Komplettverlust, um im Falle der erfolgreichen Überfahrt aus der Prämie Gewinn zu schlagen. Dieses Risiko wollte man jedoch nicht alleine tragen, so dass in den allermeisten Fällen eine Gruppe von Underwritern gemeinsam ein Schiff versicherte. Nicht alle wollten oder konnten sich en Detail mit allen Informationen auseinandersetzen, sodass meist ein sehr gut informierter Underwriter eine Versicherungspolice für eine vermeintlich sichere Fahrt zeichnete, und dann viele Trittbrettfahrer ebenfalls ihr Glück versuchten und damit das Risiko teilten. ${ }^{53}$

Wenn man ausreichend Underwriter für eine Versicherungs-Police fand, war das Schiff gegen die natürlichen und menschlichen Gefahren auf See versichert und die Besitzer befanden sich in der komfortablen Position, das Schiff auszusenden. Solche Versicherungen gegen Risiken auf einer langen Schiffsreise waren insbesondere eine der Grundlagen des Handels mit Partnern in Asien und anderen weit entfernten Gebieten. Ab dem 18. Jahrhundert wuchs der Handel über die Weltmeere unter anderem durch diese Absicherung immer

51 Thomas Southcliffe Ashton: An economic history of England. The 18th Century, London 1977, S. 72.

52 Kingston, Marine, S. 386.

53 Vgl. ebd. Die Aufteilung hing natürlich von der Grösse und dem Wert eines Schiffes ab. Beliebt waren jedoch 8, 16, 32 oder 48 Underwriter. Vgl. weiter: Violet Barbour: Marine risks and insurance in the seventeenth century, in: Journal of Economic and Business History 1 (1928-1929), S. 561-596, hier S. 569f. 
mehr an. ${ }^{54}$ Der Gefahrenraum Meer und das dort vorhandene Risiko wurde durch diese Informationen zunehmend kalkulierbarer und konnte in Zahlen berechnet werden. Mit dem Umzug in die Royal Exchange entstand so ein vielversprechender trading point für maritime Informationen, in dem die gesammelten Daten von allen Beteiligten als Ware wahrgenommen wurden. Die Quantität dieser Datensammlung spricht für sich und lässt auch heute noch erahnen, welche Organisationsstruktur im Hintergrund vorhanden sein musste, um diese zu verarbeiten: Innerhalb von zwei Monaten des Jahres 1829 erreichten 3558 Shipping Lists und 627 individuelle Briefe von Agenten oder Subscribern London, deren Informationen bewertet und verarbeitet wurden, um anschließend in 30 Lists herausgegeben zu werden.

Die Lloyd's List stellt ein Netzwerk von Versicherten, Versicherern, Anlegern und Objekten dar. Mit dem großen Wachstum des britischen Seehandels (zwischen 1780-1850 ca. um den Faktor 10) und den Telegraphen als schnellerem Kommunikationsmedium bekam dieses Netzwerk einen globalen Maßstab. In einem derart kapitalintensiven Bereich wie dem der Versicherungen mussten stets möglichst genaue Informationen vorliegen, um das eigene Risiko kalkulieren zu können. Ein Vorsprung bei der Ware Information bedeutete oft höhere Gewinne.

Durch eine eigene Informationsverwaltung war ein Kaufmann oder Reeder in London auch über die Vorgänge rivalisierender Händler oder Handelskompanien informiert. Es entwickelte sich ein ganz neuer Zugang, mit Informationen umzugehen. Indem die bereits zusammengestellten Daten der Lloyd's List individuell weiterverarbeitet wurden, konnten sie ihren Konkurrenten zuvorkommen. Dies erforderte jedoch zusätzliche Informationen und Beziehungen, um beispielsweise die Schiffsnamen bestimmten Eignern zuzuordnen oder die Auftraggeber einzelner Kapitäne zu verfolgen. Hierfür wurden neue Publikationen benötigt. Neben den Lloyd's List und dem Lloyd's Register entstanden (zu einem späteren Zeitpunkt) beispielsweise die Lloyd's Shipping Lists mit den Klassifikationen der einzelnen Schiffe samt ihren Kapitänen mit deren Werdegängen und - aufbauend auf den Daten der Lists - kartengestützte Register der havarierten Schiffe und Wracks. Diese Verluste an Schiffen und Ladungen stellten Brüche im normalen Alltagsgeschäft dar, welche die Notwendigkeit eines Tools wie der Lloyd's List untermauerten, um künftige Gefahren zu minimieren.

Die Sammlung und Verarbeitung solch riesiger Datenmengen innerhalb weniger Tage erforderte eine erhebliche logistische Leistung, die kontinuierlich

54 Verschiedene Arbeiten unterstützen diese Aussage, vgl. beispielhaft: Schulte, Deutsche Kaufleute, S. 331 und William Ashworth: Customs and excise. Trade, production and consumption in England 1640-1845, Oxford 2003, S. 135. 
wuchs. Im Laufe des 18. und 19. Jahrhunderts wurde London zum Handelsund Finanzzentrum Europas und die Lloyd's List wurde differenzierter und umfangreicher. Sie musste in den folgenden Jahrzehnten ihren Inhalt verändern. Das Sammeln von Informationen wurde zu einem eigenen Unternehmen. Der Ablauf und die Veränderung der Aggregation durch technologische Neuerungen, etwa die Telegraphie, oder das verbesserte Postwesen, aber auch der langsame Übergang von Segel- zu Dampfschiffen bis hin zur Nutzung der Schiffspropeller wirkte auf Reisewege, Kommunikationszeiten und letztlich auf die Standards der Informationssammlung ein. Wie schnell oder langsam passte sich der Komplex der Lloyd's seiner Zeit an? Mit der Zeit wuchs der Handel über Häfen mit direkter britischer Kontrolle hinaus. Informationen aus weit entlegenen Gebieten wurden über mehrere Stationen geleitet, die nicht immer genau und verlässlich arbeiteten. Man konnte nicht wie bisher allein auf die generierten Listen vertrauen, sondern benötigte weitere Informationsquellen.

Durch die Zunahme der weltweiten Handelsschifffahrt und der damit verbundenen Häufung von beschädigten oder gar zerstörten Schiffen und Waren verlangten die Kaufleute bei Lloyd's also immer wieder eine Anpassung der Informationsbeschaffung und deren Weiterverarbeitung. Mit der Zeit wurde vor allem in Übersee oftmals versucht, durch Vortäuschung falscher Tatsachen die Versicherungssummen zu erschleichen. Bald wurde klar, dass die Underwriter vertrauenswürdige Personen vor Ort benötigten, um vor Betrugsfällen geschützt zu werden und um auch eine Ansprechperson anbieten zu können, wenn es Probleme gab. Ab 1811 entwickelte sich ein noch heute aktives, weltumspannendes Agentennetzwerk, dessen Anfänge im Folgenden beschrieben werden.

\section{Die Agenten der Lloyd's}

Als zweite Professionalisierungsphase kann der Aufbau eines weltweiten Agentennetzwerkes identifiziert werden, welches eine viel detailgenauere Informationssammlung erlaubt als sie durch Sound- und Marine Lists möglich gewesen wäre. Ein Agent der Lloyd's ist damals wie heute der Ansprechpartner in einem Versicherungsfall vor Ort. Er regelt die Kommunikation mit den Eignern, Beteiligten und der Lloyd's. Weiter prüft er, ob tatsächlich ein Versicherungsschaden vorliegt. Obwohl historische Darstellungen von Lloyd's selbst diese Etablierung erst auf Mitte des 19. Jahrhunderts datieren, konnte im Archiv eine Agentenliste für das Jahr 1821 bestätigt werden. ${ }^{55}$ Einzelne Nennungen

$55 \mathrm{Zu}$ vergleichen wären hier neben den diversen Jubiläumsbänden auch die historischen Bereiche der Homepages von Lloyd's of London oder Lloyd's List. 
innerhalb der Protokolle des General Meetings im August 1800 lassen sogar auf eine noch frühere Organisation schließen:

$»$ Resolved, that these Resolutions be published in the Public Papers, as those of the Underwriters at Lloyds, and that printed Copies of the sentence pronounced by Judge Grose be circulated among the Subscribers to this House and sent to the Agents at the Outer Ports. $\aleph^{56}$

Vermutlich waren die ersten Agenten zunächst nur einfache Lieferanten für die Shipping Intelligence und weiterer Informationen. Unter diesem Begriff sind die Daten zusammengefasst, welche bisher aus den Sound- und MarineLists extrahiert wurden. Nunmehr war ein Agent für deren Beschaffung zuständig, und dabei wesentlich zuverlässiger als frühere Quellen. Daneben sammelten sie eine neue Art von Information - die Speakings. Auch wenn die Weltmeere eine riesige Ausdehnung erreichen, bewegen sich die Schiffe doch auf mehr oder minder einheitlichen Routen. Dadurch kam es nicht selten vor, dass sich zwei Schiffe auf ihren jeweiligen Routen trafen. Ein geläufiger maritimer Begriff hierfür war to speak. Dies konnte eine tatsächlich verbale Kommunikation bedeuten, oftmals verständigte man sich jedoch mit einheitlichen Flaggensignalen. ${ }^{57}$ Lloyd's bezahlte nun Kapitäne, damit diese solche Treffen in einem zweiten Logbuch festhielten, und sämtliche Informationen wie Name und Kapitän des Schiffes, Abfahrts- und Zielhafen, Ladung und mögliche Beschädigungen notierten. Die Daten dieses Logbuchs wurden dann so schnell wie möglich einem Agenten übermittelt, damit dieser weiter an Lloyd's berichtete. Diese Praxis war nicht neu - wurde von Lloyd's jedoch professionalisiert, institutionalisiert und damit auf ein neues Level gehoben. ${ }^{58}$ Mit diesen Elementen verfügte man über eine neue Qualität von Information - der Ozean war, frei nach Roland Wenzlhuemer, nun nicht mehr eine Black Box, ein Händler konnte nun zumindest Ausschnittsweise den Reiseweg seines Schiffes nachverfolgen.

Wie sich aus den Akten erkennen lässt, befanden sich jedoch auch Agenten in teilweise recht prominenten Positionen wie der eines Konsuls, eines Gouverneurs oder eines höheren Offiziers der British Navy, also eines Vertreters der britischen Regierung im jeweiligen Hafen (siehe Abbildung 4). Wenn man

56 Lloyds of London: A List of the subscribers to Lloyd's. Also a list of the agents and a copy of their appointment and instructions, December 31, 1821, London 1822; Vgl. Minutes, MS31571001, S. 271.

57 Vgl. Seija-Riitta Laakso: Across the Oceans. Development of Overseas Business Information Transmission, 1815-1875 [Dissertation], Helsinki 2006, S. 148.

58 So berichtet Karel Davids von derartigen Aufzeichnungen bereits im 17. Jahrhundert. Vgl. Karel Davids: Sources of Knowledge. Journals, Logs, and Travel Accounts, in: Maritime history. Volume 2: The eighteenth century and the classic age of sail, in: John Hattendorf (Hrsg.), Malabar 1997, S. 79-86, hier S. 83. 
heute durch größere und in der Vergangenheit bedeutende Hafenstädte geht, findet man fast immer an verschiedenen Hauswänden die Embleme der Lloyd's-Agenten. Möglicherweise sind es sogar noch die gleichen Firmen oder Familien, welche die Aufgaben für Lloyd's übernehmen. Denn auch in Zeiten sekundenschneller Kommunikation und über GPS ortungsbarer Schiffe wünscht sich ein Reeder oder Kaufmann einen Ansprechpartner vor Ort, der sich um alle anfallende Arbeiten und Ärgernisse kümmert. ${ }^{59}$

Lloyd's nutzte ebenfalls diese Verbindungen, um eigene politische Vorhaben zu unterstützen. Um 1807 wurden im britischen Parlament einige Gesetzesvorlagen über eine erhöhte Zeichnungssteuer für maritime Versicherungspolicen lanciert, was direkte negative Auswirkungen für Lloyd's bedeutet hätte. Neben vielen anderen Bemühungen richtete Lloyd's eine Bitte an jene Subscriber, welche im Parlament saßen:

»That the Members of the City of London, and such Subscribers to this House who are Members of Parliament, be requested to watch the progress of the Bill intended to be brought in $[\ldots] \ll^{60}$.

Diese, im Übrigen teils erfolgreiche Einflussnahme, steht exemplarisch für eine Vielzahl von politischen Bemühungen durch Lloyd's of London. Neben dem Sammeln von Informationen nutzte Lloyd's sein Netzwerk also auch für andere Zwecke. Diese enge Verbindung zwischen privaten Kaufleuten und politischen Akteuren fand sich zu dieser Zeit ebenfalls in den anderen spezialisierten Kaffeehäusern Londons, gerade die Politik nutzte allerdings die gut ausgebauten Kommunikationsstrukturen bei Lloyd's im Speziellen. ${ }^{61}$ Vorstellbar ist, dass auf bereits bestehende Strukturen der East India Company zurückgegriffen wurde, um so das eigene Agentennetzwerk zu entwickeln.

\section{Ausblick}

Die Räumlichkeiten der Lloyd's wandelten sich wie vorgestellt mit der Zeit von der britischen Kaffeehauskultur zu einem knallharten Versicherungsmarkt, der aber gleichzeitig auch Umschlagplatz für Informationen und Neuigkeiten jeder

59 Vgl. Kai Umbach: Das grenzüberschreitende Geschäft in der See- und Transportversicherung von Ende des 19. Jahrhunderts bis in die 1990er Jahre. Ein internationaler Gewerbezweig auf dem Weg hin zu »globalisierten« Verhältnissen? [Dissertation], Marburg 2008, S. 14.

60 Minutes, MS31571-002, S. 302.

61 Für Wolfgang Behringer bedurfte es zur Konstituierung der politischen Öffentlichkeit neben den gut erforschten Kaffeehäusern zusätzlicher einer "soliden Infrastruktur der Kommunikation«. Diese fand sich beispielslos in den Räumlichkeiten der Lloyd's, und war zudem in eine in sich geschlossene und vertrauliche Gemeinschaft eingebettet, was den Politikern zugutekam. Vgl. Behringer, Merkur, S. 17. 
Art wurde. Je professioneller dieser Handel mit der Ware Information wurde, desto wichtiger wurde die verlässliche Lieferung und Versorgung mit dieser. Somit war man bei Lloyd's bemüht, einen sehr engen Kontakt zur Post zu pflegen, was wie vorgestellt auch im ganz örtlichen Sinne zu verstehen war. In der vorliegenden Arbeit konnte nur ein kleiner Einblick in die Verbindung mit dem postalischen Netzwerk gegeben werden. Die Bedeutung und der entscheidende Wettbewerbsvorteil konnte jedoch, so hofft der Autor, verdeutlicht werden. Im Verlauf des 19. Jahrhunderts wurden weitere Bande geknüpft, diese wurden in der vorliegenden Arbeit nur angerissen. Ziel ist es zunächst, in dieses bisher nicht weiter erforschte Thema einzuführen und einen Ausblick zu geben, wohin die Reise gehen kann, wenn weitere Quellen und Literaturbestände erarbeitet worden sind.

So blieb es natürlich nicht nur bei der britischen Post, denn Lloyd's schloss nach und nach ähnliche Verträge mit anderen Poststellen ab. Einige Zeit päter wurde sogar eine Priorisierung der Weitergabe angestrebt. Die engen persönlichen Verbindungen zum Londoner General Post Office stellten jedoch eine große Ausnahme dar. Auch kaufte man seitens Lloyd's im letzten Drittel des untersuchten Jahrhunderts sämtliche nicht mehr benötigte Signal Stations der Post auf, um die schnellere Weitergabe der Informationen durch Semaphoren und Telegraphennetzwerke zu ermöglichen.

Die enge Verzahnung zwischen Politik, Post und Versicherern stellte eine für alle Beteiligten sinnvolle Verbindung dar, welche nach Meinung des Autors einen wichtigen Pfeiler in der Aufbau- und Wachstumsphase von Lloyd's of London darstellt, ohne den sie nicht die heutige starke Dominanzposition nach einem fast 300-jährigem Bestehen innehaben würden. Eine weitere Forschung in diesem Bereich lohnt und verspricht noch viele interessante Ergebnisse auch und gerade für die historische Erforschung des Postwesens.

\section{Quellenverzeichnis}

Contracts for Supplies of Intelligence, ohne Jahr, GL, MS31648.

Lloyd's List \& Shipping Gazette. 1734-1934, 200 years of shipping news, GL, Fo Pamphlet 288.

Minutes of the Committee of Lloyd's, Dec 1771-Aug 1804, GL, MS31571-001.

Minutes of the Committee of Lloyd's, Sep 1804-Dec 1809, GL, MS31571-002.

Minutes of the Committee of Lloyd's, Jan 1810-Aug 1811, GL, MS31571-003.

Minutes of the Committee of Lloyd's, Sep 1823-Feb 1824, GL, MS31571-007.

Minutes of the Committee of Lloyd's, Sep 1824-Feb 1826, GL, MS31571-008.

Report of the Special Committee 1824, GL, 8244

Richard Chamberlain, The History Lloyd's Intelligence Unit, unpublished typescript 1942, GL, SL 64:9. 


\section{Literaturverzeichnis}

Mark Andrejevic: Infoglut. How too much information is changing the way we think and know, New York 2013.

Kenneth R. Andrews: Ships, money, and politics. Seafaring and naval enterprise in the reign of Charles I., Cambridge 1991.

William Ashworth: Customs and excise. Trade, production and consumption in England 1640-1845, Oxford 2003.

Violet Barbour: Marine risks and insurance in the seventeenth century, in: Journal of Economic and Business History 1 (1928-1929), S. 561-596.

Peter Borscheid: Systemwettbewerb, Institutionenexport und Homogenisierung. Der Internationalisierungsprozess der Versicherungswirtschaft im 19. Jahrhundert, in: Zeitschrift für Unternehmensgeschichte 51 (2006).

Peter Borscheid: Europe. Overview. in: Ders. Niels Haueter: World Insurance. The evolution of a global risk network, Oxford 2012.

Wolfgang Behringer: Im Zeichen des Merkur. Reichspost und Kommunikationsrevolution in der frühen Neuzeit (Veröffentlichungen des Max-Planck-Instituts für Geschichte 189), Göttingen 2003.

Alan Cameron, Roy Farndon: Scenes from sea and city. Lloyd's List 1734-1984, London 1984.

Centre for Port \& Maritime History: Infosheet 52, Lloyd's Marine Insurance Records, Merseyside Maritime Museum, Liverpool 2004.

Karel Davids: Sources of Knowledge. Journals, Logs, and Travel Accounts. in: Maritime history. Volume 2: The eighteenth century and the classic age of sail, in: John Hattendorf (Hrsg.), Malabar 1997, S. 79-86.

Ralph Davis: The rise of the English shipping industry. In the seventeenth and eighteenth centuries, 2. impr., Newton Abbot 1972.

Heiko Droste: The Terms of Royal Service. Post Servants' Finances, c. 1700, in: Heiko Droste (Hrsg.): Connecting the Baltic Area. The Swedish Postal System in the Seventeenth Century, Huddinge 2011, S. 123-174.

Ronald Findlay, Kevin H. O'Rourke: Power and plenty. Trade, war, and the world economy in the second millennium (The Princeton economic history of the Western world), Princeton 2009.

Sheryllynne Haggerty: `Merely for money`? Business culture in the British Atlantic, 17501815 (Eighteenth-century worlds), Liverpool 2012.

Jessica Karagöl: Girdling the Globe, networking the world. A discourse analysis of the media representation of nineteenth-century transport and communication technologies in Victorian Britain, 1838-1871 [Dissertation], Heidelberg 2013.

Christopher Kingston: Marine Insurance in Britain and America, 1720-1844. A Comparative Institutional Analysis, in: The Journal of Economic History 67 (2007).

Christopher Kingston: Adverse Selection and Institutional Change in Eighteenth Century Marine Insurance, Edinburgh 2008.

Maria Kyriakou: From discreteness to cooperation. Relational contracting in the London marine insurance market [Dissertation], London 2002. 
Seija-Riitta Laakso: Across the Oceans. Development of Overseas Business Information Transmission, 1815-1875 [Dissertation], Helsinki 2006.

Martin Lengwiler: Risikopolitik im Sozialstaat. Die schweizerische Unfallversicherung 18701970 (Industrielle Welt 69), Köln 2006.

Manuel Llorca-Jaña: The Marine Insurance Market for British Textile Exports to the River Plate and Chile. 1810-50. in: The development of international insurance, in: Robin Pearson (Hrsg.), London 2010, S. 25-36.

James T. Lloyd.: Lloyd's Post Office Directory. Of the Unites States and Canadas, with new postal laws, New York 1863.

Lloyd's Register of Shipping: Annals of Lloyd's Register. Being a sketch of the origin, constitution, and progress of Lloyd's Register of British \& foreign shipping, London 1884.

Adrian Leonard: Gresham and Defoe (Underwriters). The Origins of London Marine Insurance, London 2014.

Ikujiro Nonaka: A Dynamic Theory of Organizational Knowledge Creation, in: Organization Science 5/1 (1994), S. 14-37.

Margrit Schulte Beerbühl: Deutsche Kaufleute in London. Welthandel und Einbürgerung (1660-1818) (Veröffentlichungen des Deutschen Historischen Instituts London 61), München 2007.

Thomas Southcliffe Ashton: An economic history of England. The 18th Century. London 1977.

Kai Umbach: Das grenzüberschreitende Geschäft in der See- und Transportversicherung von Ende des 19. Jahrhunderts bis in die 1990er Jahre. Ein internationaler Gewerbezweig auf dem Weg hin zu »globalisierten« Verhältnissen? [Dissertation], Marburg 2008. 


\section{Abbildungen}

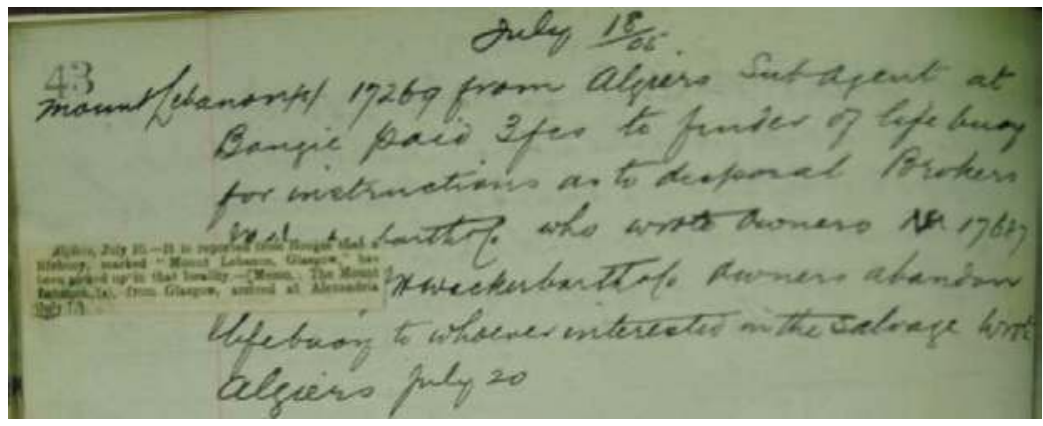

Abbildung 1: Handgeschriebener Bericht über ein auf Grund gelaufenes Schiff und der dazugehörige Eintrag in einer Lloyd's List, Minutes 1805. Courtesy of The Corporation of Lloyd's.

\section{$=$ IILOIDPS ILIST.}

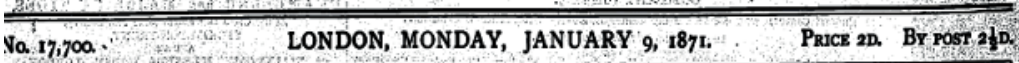

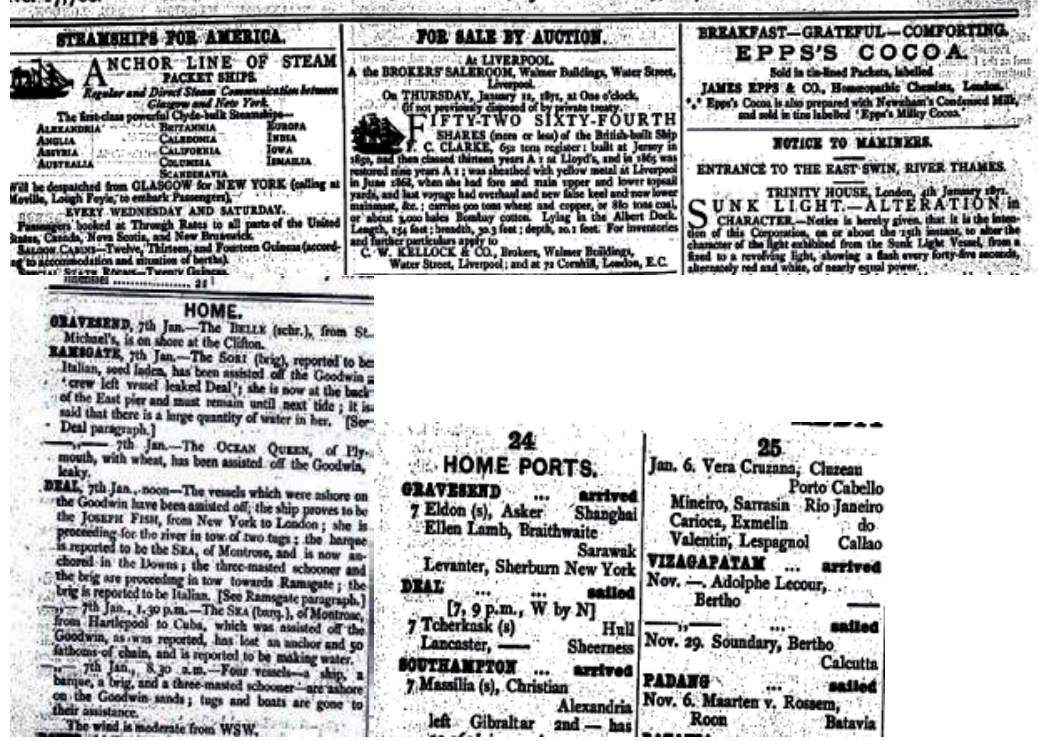

Abbildung 2: Ausschnitte aus einer Lloyd's List. Courtesy of The Corporation of Lloyd's. 


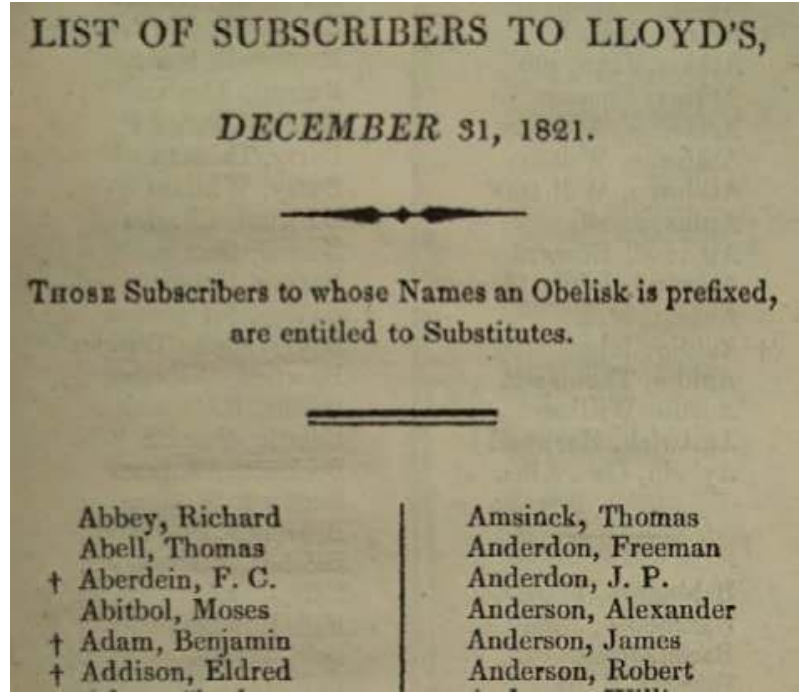

Abbildung 3: Auszug aus einer frühen publizierten Mitgliederliste der Lloyd's Subscriber, Lloyd's of London, 1822. Courtesy of The Corporation of Lloyd's.

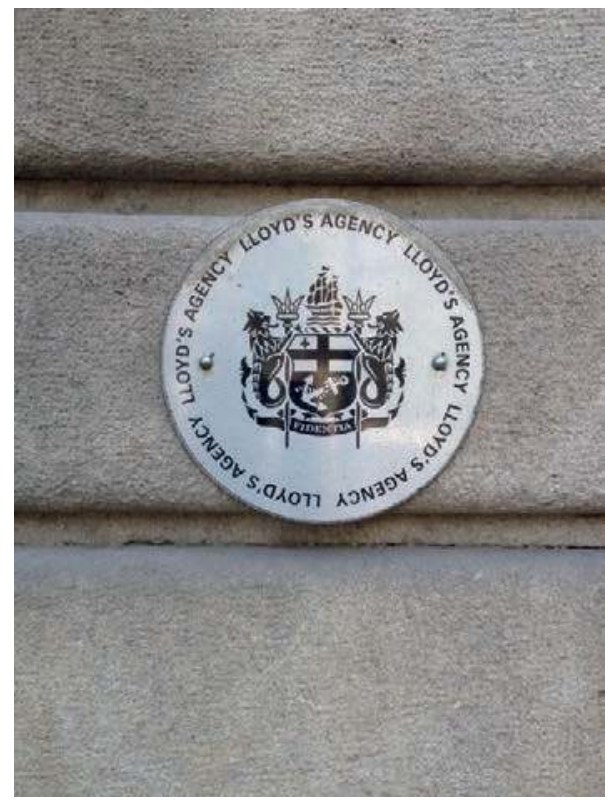

Abbildung 4: Die Plakette einer Llyod's Agency in Barcelona. 


\section{Briefe aus Äthiopien und dem Sudan. Die Briefkommunikation zwischen europäischen Reisenden und dem Kartographen August Petermann in Gotha, 1854-1880}

\section{Einleitung}

Am 24. Mai 1874 erhielt August Petermann folgendes Telegramm aus Kairo: "Erhalte soeben Telegramm aus Khartum dass / Nachtigall von Batigoh abgereist / wohlbehalten in Darfur angekommen ist « (siehe Abbildung 1). ${ }^{1}$ Absender des Telegramms war Gustav Travers, seit 1872 Vizekonsul des Deutschen Reichs. ${ }^{2}$ Damit setzte er auf dem damals schnellsten Weg August Petermann in Gotha über diese offenbar außergewöhnliche Nachricht in Kenntnis. Die Besonderheit dieses Vorgangs zeigt sich bereits darin, dass es sich um das einzige Telegramm aus Afrika handelt, das in der Korrespondenz des Gothaer Kartographen mit europäischen Reisenden, die in Äthiopien und im Sudan ${ }^{3}$ unterwegs waren, überliefert ist. ${ }^{4}$ Mit dem Erhalt dieses Telegramms partizipierte Petermann an den neusten sich gerade entwickelnden kommunikativen Strukturen.

Den Einfluss von Printmedien und elektronischen Massenmedien auf Gesellschaft und Politik seit dem 19. Jahrhundert schätzt die Forschung vielfach als so bedeutend ein, dass er als ein zentrales »Paradigma einer Gesellschaftsgeschichte der Moderne« bezeichnet wird. ${ }^{5}$ Ausgehend von den soziologischen

1 Sammlung Perthes: SPA ARCH PGM 195, Bl. 65.

2 Ernennung der Deutschen Konsuln, 12. März 1872, in: Deutsches Reichsgesetzblatt, Nr. 8, Berlin 1872, S. 66, URL: https://de.wikisource.org/wiki/Ernennung_zu_Deutschen_Konsuln. Vom_12._März_1872 (Letzter Zugriff: 16.09.2019).

3 Als Sudan wurde im 19. Jahrhundert die gesamte Region südlich der Sahara bezeichnet.

4 Sammlung Perthes: Bestand SPA ARCH PGM.

5 Habbo Knoch, Daniel Morat: Medienwandel und Gesellschaftsbilder 1880-1960. Zur historischen Kommunikologie der massenmedialen Sattelzeit, in: Dies. (Hrsg.): Kommunikation als Beobachtung. Medienwandel und Gesellschaftsbilder 1880-1960, München 2002, S. 9-33, hier S. 14. Siehe auch: Jörg Requate: »Kommunikationsmedien und Gesellschaft«, in: Reinhard Sie- 
Theorien des Kommunikationswissenschaftlers Armand Mattelart zur globalen Vernetzung entwickelte Roland Wenzlhuemer eine Theorie kommunikativer Räume, die mit der Ausbildung und Nutzung neuer Kommunikationstechnologien und deren weltweiter Vernetzung entstanden. ${ }^{6}$ Nach Mattelart und Wenzlhuemer war der Ausgangspunkt dieser neuen Entwicklungen der Ausbau des globalen Telegraphennetzwerks, das zu einer neuen Qualität der zeitlichen und räumlichen Dimension globaler Kommunikation führte. Ebenso betrachtet Hartmut Rosa den Ausbau des globalen Telegraphennetzwerks als Teil eines Prozesses der Beschleunigung der Kommunikation und damit auch der Lebensformen in der modernen Gesellschaft, der dem sozialen Zusammenleben ganz neue Strukturen verleiht. ${ }^{7}$

In der zweiten Hälfte des 19. Jahrhunderts war das Versenden von Telegrammen noch ein außergewöhnlicher Akt, denn das internationale Telegraphennetzwerk war im Aufbau begriffen und Telegramme waren sehr teuer. ${ }^{8}$ Zwar eröffnete dieses globale Netzwerk im Ansatz bereits die Möglichkeiten zur Schaffung neuer Kommunikationsräume, aber bis ins 20. Jahrhundert hinein dominierte noch der Brief die alltägliche interpersonale Kommunikation und die Zahl der versandten Briefe nahm beständig zu. ${ }^{9}$

Auch europäische Forschungsreisende nutzten vor allem die Möglichkeiten der brieflichen Kommunikation. Während sie zwischen 1854 und 1880 durch Äthiopien und den Sudan - sowie durch andere Regionen Afrikas - reisten, standen sie mit ihrer Familie, ihren Freunden und Kollegen in kontinuierlichem brieflichem Kontakt, und sie korrespondierten auch mit den Mitarbeitern des Verlags Justus Perthes. ${ }^{10}$ Letztere begleiteten die Reisenden mit ihrer Korrespondenz wissenschaftlich, machten deren Forschungsergebnisse noch

der, Ernst Langthaler (Hrsg.): Globalgeschichte 1800-2010, Wien/Köln/Weimar 2010, S. 439469; Ute Frevert, Wolfgang Braungart (Hrsg.): Sprachen des Politischen. Medien und Medialität in der Geschichte, Göttingen 2004.

6 Armand Mattelart: Kommunikation ohne Grenzen? Geschichte der Ideen und Strategien globaler Vernetzung, 2. überarbeitete Auflage Berlin 2007; Roland Wenzlhuemer: Globalization, communication and the concept of space in global history, in: Historical Social Research 35 (2010), S. 19-47.

7 Hartmut Rosa: Beschleunigung. Die Veränderung der Zeitstruktur in der Moderne, Frankfurt am Main 2005.

8 William Huber: Le réseau télégraphique du globe, in: Bulletin de la Société de Géographie 1873/2, S. 490-520; Mattelart, Kommunikation, S. 18-26.

9 David Vincent: The Rise of Mass Literacy. Reading and Writing in Modern Europe, Cambridge/Oxford/Malden, MA 2000, S. 3.

10 Ein Beispiel einer solchen Kommunikation wird präsentiert und kommentiert in: Reiner Prass: Forschungsreise und Wissensproduktion in Afrika in der Mitte der 19. Jahrhunderts, in: Themenportal Europäische Geschichte 2019, URL: https://www.europa.clio-online.de/essay/id/ fdae-1728 (letzter Zugriff: 28.01.2020). 
während der Reise in ihren Publikationen bekannt ${ }^{11}$ und nutzten deren Messungsergebnisse zur beständigen Aktualisierung ihrer Karten. ${ }^{12}$ Häufigkeit und Form dieser Korrespondenz wurden durch die spezifischen Strukturen der Kommunikationsnetzwerke in Afrika und Europa bestimmt. Diese Strukturen sollen im Folgenden anhand der Korrespondenz zwischen dem leitenden Kartographen des Verlags Justus Perthes, August Petermann, und mehreren Europäern, die in Äthiopien und dem Sudan unterwegs waren, erläutert werden. Es soll gezeigt werden, dass schon vor dem Ausbau eines weltumspannenden Telegraphensystems Strukturen einer globalen Kommunikation bestanden, an denen die hier vorgestellte Korrespondenz entscheidenden Anteil besaß.

Doch bevor die Strukturen dieser Kommunikationsnetzwerke ausgearbeitet werden, ist zunächst noch zu klären, warum Gustav Nachtigals Ankunft in Darfur ein so außergewöhnliches Ereignis war, dass der Vizekonsul des Deutschen Reichs Petermann telegraphisch darüber informierte. Und es sollen die Mittel der Kommunikation erläutert werden, die Gustav Nachtigal auf seiner Reise vom Tschadsee bis zum Nil zur Verfügung standen.

\section{Der historische Hintergrund: Eine Reise ins Wadaï und nach Darfur}

Mit seiner Reise durch die Region vom Tschadsee bis zum Nil ließ sich Gustav Nachtigal auf ein überaus gefährliches Abenteuer ein, das zuvor schon mehrere Male gescheitert war. So begab sich am 1. Januar 1856 der deutsche Astronom Eduard Vogel von Kuka ${ }^{13}$ ins östlich des Tschadsees gelegene Sultanat Wadaï, ${ }^{14}$ um von dort aus weiter bis zum Nil zu gelangen. ${ }^{15}$ Nach einer 26tätgigen Reise kam er nach Wara, der damaligen Hauptstadt Wadaïs, in welcher ihn Sultan Mohammed Sherif Anfang Februar 1856 ermorden ließ. ${ }^{16} \mathrm{Ab}$

11 Das betrifft vor allem die "Mittheilungen aus Justus Perthes Geographischer Anstalt über wichtige neue Erforschungen auf dem Gesamtgebiete der Geographie« (1855-1878); ab 1879 weitergeführt unter dem Titel: »Dr. A. Petermanns Mitteilungen aus Justus Perthes' Geographischer Anstalt«.

12 Vgl. Petra Weigel: Die Sammlung Perthes Gotha, Forschungsbibliothek Gotha, in: Patrimonia 254, Berlin 2011.

13 Kuka war damals die Hauptstadt des Sultanats Bornu. Sie liegt westlich des Tschadsees; heute gehört es zur Provinz Borno im Nordosten Nigerias.

14 Zum Wadaï im 19. Jahrhundert siehe: Mario J. Azevedo: Roots of Violence. A History of War in Chad, Amsterdam 1998, S. 26-28.

15 Hermann Wagner (Bearb.): Schilderung der Reisen und Entdeckungen des Dr. Eduard Vogel in Central-Africa in der großen Wüste, in den Ländern des Sudan, nach den Originalquellen bearbeitet, Leipzig 1860, S. 309.

16 Dietmar Henze: Enzyklopädie der Entdecker und Erforscher der Erde, Bd. 5, Neuausgabe Darmstadt 2011, S. 425. 
1857 kursierten in der Region erste Gerüchte über Vogels Tod, ${ }^{17}$ aber die darauf erfolgenden Versuche, sein Schicksal endgültig aufzuklären und seine wissenschaftlichen Aufzeichnungen zu sichern, schlugen weitgehend fehl.

Werner Munzinger und Gottlob Theodor Kinzelbach versuchten 1862 im Rahmen der deutschen Afrikaexpedition von Khartum aus über Darfur in das Sultanat Wadaï einzureisen. Sie kamen aber nur bis nach El Obeid, der Hauptstadt der westsudanesischen Provinz Kordofan, denn der Sultan Darfurs erlaubte ihnen die Durchreise nicht. Darüber hinaus war es auch ungewiss, ob der Sultan des Wadaï ihnen die Einreise gestatten würde. ${ }^{18}$ Ein Jahr später wurde Karl Moritz von Beurmann bei seinem Versuch, von Westen aus in das Wadaï einzureisen, an der Grenze getötet. ${ }^{19}$

Dank seiner guten Kontakte zu den arabischen Händlern erhielt Munzinger in El Obeid die Bestätigung, dass Vogel im Wadaï ermordet worden war, er konnte aber nichts genaueres über die Umstände und den Zeitpunkt von Vogels Tod erfahren. ${ }^{20}$ Erst Nachtigal gelang es, dessen Schicksal endgültig aufzuklären. Von Sultan Ali erfuhr er, dass Alis Vater Mohammed Sherif Vogel ermorden ließ. Sultan Mohammed Sherif verdächtigte ihn, ein Spion zu sein und er habe sich auch sonst verdächtig verhalten: Vogel habe nur schlecht arabisch gesprochen und daher wenig Gäste empfangen, er habe sich ausschließlich von Hühnereiern ernährt - was doch kein anständiger Mensch zu tun pflege - und er habe nicht mit einer Feder, sondern einem Stab geschrieben. Die Notizen und Tagebücher Vogels konnte auch Nachtigal nicht erhalten. ${ }^{21}$

Gustav Nachtigal wusste also durchaus, dass er sich auf ein lebensgefährliches Abenteuer einließ. Doch bereits mit seiner Reise nach Afrika hatte er sich auf ein solches Wagnis eingelassen, denn zahlreiche Europäer fanden bei derartigen Unternehmungen im Innern des Kontinents den Tod. ${ }^{22}$

17 Wagner, Schilderungen, S. 315.

18 Werner Munzinger's Bericht über seine und G. Th. Kinzelbach's Reise nach El Obed 1862, in: Mittheilungen, Bd. 9 (1863), S. 183-190; Ein Brief von Th. Kinzelbach aus El Obed, der Hauptstadt von Kordofan, in: Mittheilungen, Bd. 9 (1863), S. 217-225.

19 Moriz v. Beurmann's Tod nebst Übersicht seiner Reise (1861-1863) so wie derjenigen von Overweg (1850-1852), Vogel (1853-1856) und Steudner (1861-1863), in: Mittheilungen, Bd. 10 (1864), S. 25-30.

20 »Nachrichten über Ed. Vogel's Schicksal von der Munzinger’schen Expedition «, in: Mittheilungen, Bd. 8 (1862), S. 346-350; zu den Kontakten Munzingers in El Obeid siehe: Kinzelbach, Brief.

21 Gustav Nachtigal: Saharâ und Sûdân. Ergebnisse Sechsjähriger Reisen in Afrika, Bd. 3, Leipzig 1889 , S. $171-173$.

22 Vgl. Wolfgang Struck: Karten-Legenden. August Petermanns Verschollene, in: Irina Gradinari, Dorit Müller, Johannes Pause (Hrsg.): Versteckt - Verirrt - Verschollen. Reisen und NichtWissen, Wiesbaden 2016, S. 69-83. 
Prinzipiell war eine Reise im Innern Afrikas nur dann möglich, wenn ihr die Herrscher der betreffenden Regionen zustimmten, ${ }^{23}$ und so benötigte Nachtigal auch für die Realisierung seiner Reisepläne vorab die offizielle Erlaubnis der beiden Sultane von Wadaï und Darfur. Hierbei unterstützte ihn Scheich Omar von Bornu. Er sandte Boten an die beiden Sultane, um für seinen Gast Gustav Nachtigal diese Erlaubnis zu erbitten. Am 10. Januar 1873 erschien der Bote Sultan Alis von Wadaï mit Briefen, in denen er Nachtigal die Einreise erlaubte und ihm seinen Boten Otmân Ûled als Reisebegleiter zur Verfügung stellte. ${ }^{24}$ Diese als intermedairies bezeichneten Boten, Führer, Übersetzer und andere Helfer waren wichtige Vermittler zwischen den Reisenden und der örtlichen Bevölkerung, ohne die solche Unternehmungen unmöglich waren, ${ }^{25}$ und in den darauf folgenden Monaten wurde Otmân Ûled auch wirklich Nachtigals wichtigste Bezugs- und Vertrauensperson. Doch er wartete noch, bis in der zweiten Februarhälfte auch der Bote Sultan Muhammad al-Hasayns aus Darfur mit positiven Nachrichten kam. Daraufhin machte sich Nachtigal zu Beginn des Monats März 1873 auf den Weg von Kuka nach Abesche, der Hauptstadt des Wadaï. Die Distanz zwischen beiden Orten schätzte er auf ca. $1000 \mathrm{~km}$, die eine Karawane gewöhnlich innerhalb von 28 bis 34 Tagen zurücklegte. ${ }^{26}$

Sultan Ali empfing Nachtigal mit großer Offenheit und Freundlichkeit, zumal er sich von seinem Gast Informationen über die umliegenden Territorien sowie über europäische Staaten und deren militärische Stärke erhoffte. ${ }^{27}$ Problematisch blieb allerdings die feindselige Haltung der Bewohner des Wadaï, vor deren Übergriffen ihn nur der ausdrückliche Schutz des Sultans bewahrte. ${ }^{28}$ Diese Feindseligkeit gegenüber Fremden, die auch Alis Vater Mohammed Sherif unterstützt hatte, schilderte Nachtigal als das wesentliche Problem des Sultanats Wadaï, das zur weitgehenden Isolierung des Landes geführt hatte. ${ }^{29}$ Aus diesem Grund mieden sogar arabische Händler das Land und somit war jeglicher Handel mit den Nilländern und dem Norden Afrikas unterbrochen.

23 Vgl. Dane Kennedy: The Last Blank Spaces. Exploring Africa and Australia, Cambridge, Mass./London 2013, S. 100.

24 Nachtigal, Saharâ und Sûdân 3, S. 18.

25 Vgl. Benjamin N. Lawrance, Emily Lynn Osborn, Richard L. Roberts (Hrsg.): Intermediaries, Interpreters, and Clerks. African Employees in the Making of Colonial Africa, Madison, Wisconsin 2006; Felix Driver, Lowri Jones: Hidden Histories of Exploration. Researching the RGSIGB Collections, London 2009; Volker Matthies: Im Schatten der Entdecker. Indigene Begleiter europäischer Forschungsreisender, Bonn 2018.

26 Nachtigal, Saharâ und Sûdân 3, S. 23.

27 Nachtigal, Saharâ und Sûdân 3, S. 58.

28 Nachtigal, Saharâ und Sûdân 3, S. 59 f.

29 Hierbei musste sich Nachtigal aber auf die Aussagen Sultan Alis und weiterer Informanten verlassen. 
Sultan Ali versuchte diese Handelsaktivitäten wieder zu beleben und einige arabische Händler kamen nun wieder nach Abesche..$^{30}$ Auf diesem Weg erhielt Nachtigal auch Post durch einen arabischen Händler namens Mohammed Zommit zugestellt. Allerdings verzögerte dieser die Herausgabe der Briefe an Nachtigal um einige Tage und schließlich musste er sich energisch gegenüber Zommit durchsetzen, damit dieser Nachtigal die Briefe und eine damit übersandte Summe von 350 Talern übergab. ${ }^{31}$ Aus Nachtigals Schilderung geht deutlich hervor, dass die Übermittlung von Nachrichten und Postsendungen in dieser Region an einzelne Personen gebunden war. Solange aber Karawanen nicht regelmäßig aus Tripolis oder vom Nil ins Wadaï kamen, war kein regelmäßiger Austausch von Nachrichten und Postsendungen möglich.

Die Weiterreise verzögerte sich erheblich, denn Sultan Muhammad alHasayn von Darfur starb 1873. Nachtigals Reisegefährten wollten abwarten, bis die Herrschaftsnachfolge in Darfur geklärt war und al-Hasayns Sohn Ibrahim Qarad seine Herrschaft gesichert hatte. Zudem kursierten Gerüchte, dass Ägypten das politisch geschwächte Darfur erobern wolle. ${ }^{32}$ Trotz der zunehmenden politischen Spannungen zwischen Darfur und Ägypten reisten schließlich Nachtigal und seine Reisegefährten am 17. Januar 1874 nach Darfur ab und am 7. März 1874 erreichten sie El Fascher, die Hauptstadt des Sultanats. ${ }^{33}$ Gleichwohl dauerte es noch über zwei Monate, bis August Petermann in Gotha das Telegramm mit der Nachricht von Nachtigals Ankunft erhielt. Diese Verzögerung war eine Folge der spezifischen Kommunikationskanäle, die Nachrichten aus dem Sudan bis in eine mitteldeutsche Stadt durchliefen.

\section{Wege der Briefkommunikation zwischen Äthiopien, dem Sudan und Deutschland 1848-1880}

Eine systematische Durchsicht der Korrespondenz von elf Forschungsreisenden, die zwischen 1848 und 1880 in der Region zwischen Niger und Rotem Meer unterwegs waren, ergibt eine große Vielfalt von Themen, über die sie in

30 Nachtigal, Saharâ und Sûdân 3, S. 61.

31 Nachtigal, Saharâ und Sûdân 3, S. 166f., 174.

32 Zur damaligen politischen Situation in Darfur und zur 1874 erfolgenden Eroberung des Landes durch Ägypten siehe M. W. Daly: Darfur's Sorrow. A History of Destruction and Genocide, Cambridge usw. 2007, S. 34-39; R. S. O'Fahey: The Darfur Sultanate. A History, London 2008, S. 261-274.

33 Nachtigal, Saharâ und Sûdân 3, S. 300f.; 336f. Dieser Karawane hatten sich Sklavenhändler angeschlossen, die auf verschlungenen Wegen ihre Ware in Richtung Ägypten schmuggelten, wo jeder Sklavenhandel mittlerweile offiziell verboten war. Auch Nachtigals Begleiter waren im Sklavenhandel tätig. 
ihren Texten berichteten. ${ }^{34}$ In erster Linie informierten sie ihre Kollegen, Freunde und Verwandten über den bereits zurückgelegten Reiseweg und über ihre weiteren Reisepläne. Zudem teilten sie ihnen Ergebnisse wissenschaftlicher Beobachtungen und Messungen mit. Darüber hinaus berichteten sie über Reisebedingungen, wie die außergewöhnlichen Belastungen durch das ungewohnte Klima oder ihre Abhängigkeit vom Wohlwollen lokaler Machthaber. ${ }^{35}$

Diese Briefwechsel erfolgten nicht wöchentlich oder gar täglich, sondern über längere Intervalle von mehreren Wochen oder zeitweise auch Monaten hinweg. Gelangten über einen längeren Zeitraum keine Briefe mehr nach Europa, galt dies als Hinweis für den wahrscheinlichen Tod des Reisenden. ${ }^{36}$ Doch längere Unterbrechungen der Korrespondenz waren auch der politischen Situation vor Ort geschuldet. So konnte Heinrich Barth während seines Aufenthalts in Timbuktu aufgrund der dortigen politischen Situation keine Briefe nach Europa senden. ${ }^{37}$

Der wesentliche Grund für das lange Ausbleiben von Nachrichten aus Äthiopien und dem Sudan war jedoch die spezifische Ausformung der Kommunikationswege in diesem Teil Afrikas. Im Unterschied zu den europäischen Staaten, in denen das Postwesen einen kontinuierlichen Briefverkehr durch seine eigenständigen Übermittlungskanäle ermöglichte, war die Vermittlung von Nachrichten in den hier behandelten Regionen Afrikas auf die Nutzung der Infrastruktur des Karawanenhandels angewiesen: Die Reisenden vertrauten ihre Briefe einem Händler an, der in einer Karawane durch ihren Aufenthaltsort kam. Dieser Händler brachte den oder die Briefe an einen zentralen Punkt innerhalb des afrikanischen Handels- und Kommunikationsnetzwerks, wie Khartum, die am Roten Meer gelegene Hafenstadt Massaua oder nach Tripolis. ${ }^{38}$ Von Khartum oder Massaua aus wurden die Briefe über Kairo nach Alexandria und von dort nach Europa gebracht. Umgekehrt ließen die Reisenden

34 Archiv der Sammlung Perthes Gotha: Signaturen SPA ARCH PGM 039 (Heinrich Barth); SPA ARCH PGM 323 (Wilhelm von Harnier); SPA ARCH PGM 277 (Wilhelm Junker); SPA ARCH PGM 366 (John Wilhelm von Müller); SPA ARCH PGM 337 (Adolf Overweg); SPA ARCH PGM 16 (Gerhard Rohlfs); SPA ARCH PGM 354 (Georg Schweinfurth); SPA ARCH PGM 62 (Theodor von Heuglin); SPA ARCH PGM 66 (Gottlob Theodor Kinzelbach); SPA ARCH PGM 72 (Werner Munzinger); SPA ARCH PGM 617 (Emin Pascha).

35 Eine genaue Analyse dieser Quellen verfolgt der Autor zurzeit in seinem Projekt über Selbstzeugnisse von Europäern, die in den 1840er - 1880er Jahren im östlichen Sudan und in Äthiopien unterwegs waren.

36 August Petermann: Die Expedition nach Central Africa II. Dr. H. Barths Rückreise von Timbuktu nach Kano, vom 8. Juli bis 17. Oktober 1854, in: Mittheilungen, Bd. 1 (1855), S. 85-89, hier S. 85; Heinrich Barth: Reisen in Nord- und Central-Africa in den Jahren 1859 bis 1855, Bd. 5, Gotha 1858, S. 144.

37 Zur Situation Heinrich Barths in Timbuktu und seinen Möglichkeiten, mit der Außenwelt in Kontakt zu treten, siehe: Barth, Reisen, Bd. 4, S. 446-472.

38 Prass, Forschungsreisen; Barth, Reisen, Bd. 4, S. 469. 
ihre aus Europa kommende Post in bestimmte Handels- und Kommunikationszentren schicken. Als 1848 Johann Wilhelm von Müller nach Khartum kam, fand er hier Briefe seiner Familie vor, ${ }^{39}$ und ebenso ließen die Mitglieder der deutschen Afrikaexpedition 1861/62 die an sie adressierten Briefe nach Khartum senden. ${ }^{40}$ Auch Emin Pascha, der ab 1876 im Südsudan lebte und von 1878 bis 1885 Gouverneur der Provinz Äquatoria war, ${ }^{41}$ ließ seine Post nach Khartum senden, von wo aus sie mit dem Dampfschiff den Weißen Nil hinauf zum seinem Wohnort Lado gebracht wurde. ${ }^{42}$

Vielfach erfolgte die Vermittlung von Nachrichten auch über Boten. Sie brachten entweder Briefe persönlich zu ihren Adressaten, oder sie überbrachten ihre Nachrichten mündlich. Letzteres war bei einer Nachricht eines afrikanischen Herrschers möglich. Adolf Overweg wies darauf hin, dass er in der Region westlich des Tschadsees ein Botensystem zwischen Mursuk und Bornu nutzte, und bei seinem Aufenthalt in Timbuktu war Heinrich Barth vollkommen auf die Dienste von Boten angewiesen. ${ }^{43}$ Ebenso erfolgte die Kommunikation im nördlichen Äthiopien zu einem ganz wesentlichen Teil durch Boten, wie aus der Reisebeschreibung Theodor von Heuglins hervorgeht, ${ }^{44}$ und Gustav Nachtigal informierte sich im Wadaï mit Hilfe von Karawanenhändlern und Boten über die aktuelle Situation im Sultanat Darfur. ${ }^{45}$ Ein regelmäßiges und vor allem zuverlässiges Nachrichtensystem existierte nicht.

Die Brieflaufzeiten konnten sich erheblich verzögern. So unterbrachen sich regelmäßig bildende Pflanzenbarrieren immer wieder monatelang die Schiffsverbindungen auf dem Weißen Nil. ${ }^{46}$ In der Sahara und in der südlich sich anschließenden Region westlich des Tschadsees war die Verbindung noch schwieriger: 1851 schrieb Adolf Overweg an seine Familie, dass die letzten Briefe, die er von ihnen erhalten hatte, bereits ein Jahr alt seien, und ein Paket von Briefen an Heinrich Barth verblieb zwei Monate in A'ssauād in der Nähe

39 Sammlung Perthes: SPA ARCH PGM 366, Bl. 20 und Bl. 44 v.

40 Prass, Forschungsreisen.

41 Christian Kirchen: Emin Pascha. Arzt - Abenteurer - Afrikaforscher, Paderborn 2014, S. 81-91.

42 Sammlung Perthes: SPA ARCH PGM 617/2, Bl. 1-4: Brief Emin Pascha an August Petermann, Lado 26. Mai 1876.

43 Sammlung Perthes: SPA ARCH PGM 337, Bl. 3: Brief Adolf Overwegs an Carl Ritter, Selufiet im Lande Ahier, 28. Aug. 1850; Barth, Reisen, Bd. 4, S. 469; Barth, Reisen, Bd. 5, S. 143 f.

44 Theodor von Heuglin: Reise nach Abessinien, den Gala=Ländern, Ost=Sudán und Chartúm in den Jahren 1861 und 1862, Jena 1868, S. 140 f., 157.

45 Nachtigal, Saharâ und Sûdân, S. 87-89, 159, 496 f.

46 Sammlung Perthes: SPA ARCH PGM 617/2, B1. 29-30: Brief Emin Bey an August Petermann, Lado 15. Sept. 1879; Wilhelm Götze: Die Verkehrswege im Dienste des Welthandels. Eine historisch-geographische Untersuchung, Stuttgart 1888, S. 776. Zur schwierigen Beseitigung der immer wieder neu entstehenden Pflanzenbarrieren siehe: Ernst Marno: Ueber die Pflanzenbarren im oberen Weißen Nil, in: Mittheilungen der Geographischen Gesellschaft in Wien 1880, S. 401-409. 
von Timbuktu, bis er es im Mai 1854 erhielt. ${ }^{47}$ Zwanzig Jahre später konnte der Informationsfluss weiterhin spärlich bleiben, wie die Geschichte Gustav Nachtigals zeigt. Im Wadaï war er von allen regelmäßigen Verbindungen zu seinen europäischen Korrespondenzpartnern abgeschnitten und erst als eine Karawane aus Tripolis nach Abesche kam, brachte ein Händler ihm Briefe und Geld von dort mit. In Darfur war Nachtigal ebenfalls auf die Verbindung mittels Karawanenhändler angewiesen, ${ }^{48}$ was auch die Verzögerung der Nachricht von Nachtigals Ankunft erklärt. Nachdem er El Fascher am 7. März 1874 erreicht hatte, brachte wahrscheinlich einer dieser Karawanenhändler ${ }^{49}$ diese Nachricht nach Khartum, von wo sie dann am 24. Mai 1874 August Petermann in Gotha per Telegramm erreichte.

In dem seit 1821 von Ägypten eroberten Teil des Sudan waren dagegen die Kommunikationsnetze besser ausgebaut. Von Khartum aus gab es eine Postverbindung den Nil hinunter bis nach Kairo, und ab Sommer 1871 existierte auch eine Telegraphenverbindung, die von Khartum den Nil entlang über Assuan nach Alexandria führte. ${ }^{50}$ In den 1870er Jahren wurde mit dem Bau einer Eisenbahn von Kairo nach Khartum begonnen, aber erst nach dem Ende der Mahdi-Herrschaft durch die britische Eroberung des Sudan 1898 wurde Khartum an das ägyptische Eisenbahnnetz angeschlossen. ${ }^{51}$

Eine weitere Möglichkeit bestand darin, die Post von Khartum aus auf den Karawanenstraßen zu den am Roten Meer gelegenen Hafenstädten Suakin und Massaua zu schicken. Im Roten Meer führte 1863 eine ägyptische Schifffahrtslinie an der afrikanischen Küste des Roten Meers entlang von Suez direkt über Casseyr und Suakin nach Massaua. ${ }^{52}$ Zudem existierte ein dichtes Netz von Schiffsverbindungen für Mekka-Pilger, das zwischen 1853 und 1868 auch Werner Munzinger, Georg Schweinfurth und Theodor von Heuglin für ihre Reisen von Suez nach Massaua nutzten. Sie fuhren von Suez aus an der Küste der Arabischen Halbinsel entlang bis nach Djedda. Von dort nahmen sie ein Schiff, das von Djedda direkt westlich nach Suakin und von dort an der afrika-

47 Sammlung Perthes: SPA ARCH PGM 337, Bl. 15-15: Brief Adolf Overwegs an seine Eltern und seine Schwester, Zelt am Ufer des Tschadsees, 26. Juni 1851.

48 Nachtigal, Saharâ und Sûdân, S. 166 f., 345.

$49 \mathrm{Zu}$ diesem Punkt äußert sich Nachtigal in seiner Publikation nicht.

50 Georg Schweinfurth: Im Herzen von Afrika. Reisen und Entdeckungen im centralen Aequatorial-Afrika während der Jahre 1868 bis 1871, Bd. 2, Leipzig/London 1874, S. 503 f.

51 Colette Dubois: Morphologies de Khartoum: conflits d'identité (1820 - début du XXe siècle), in: Hervé Bleuchot, Christian Delmet, Derek Hopwood (Hrsg.): Sūdān. History, identitiy, ideology. Histoire, identités, idéologies, Aix-en-Provence/Oxford 1991, S. 13-32, hier S. 29.

52 Sammlung Perthes: SPK-10-IV.D C-17: Carte des Voies de Communications établis dans le monde entier au moyen de la vapeur et de l'électricité. Dressée d'après les documents officiels les plus récents par Anatole Chatelain, Ex-Chef du Bureau de la Statistique au Ministère des Affaires Étrangères, Paris 1863. 
nischen Küste entlang nach dem weiter südlich gelegenen Massaua fuhr. ${ }^{53}$ Bis zur Eröffnung des Suezkanals 1869 wurde der Postverkehr im Roten Meer vielfach durch arabische Seefahrer gewährleistet, aber 1864 existierte bereits eine Postdampfschifflinie von Suez nach Aden (siehe Faltblatt 2). ${ }^{54}$

Tripolis wurde in den 1860er Jahren noch von regionalen Schiffen mit Malta und Alexandria verbunden. ${ }^{55}$ Dagegen war Alexandria bereits in der Mitte des 19. Jahrhunderts vollständig in das europäische Kommunikationsnetz integriert (siehe Abbildung 3). Spätestens ab 1864 fuhren regelmäßig englische, französische und österreichische Postschiffe von Southampton, Marseille und Triest in die ägyptische Hafenstadt. Von Alexandria ging es auf dem Landweg über Kairo nach Suez. ${ }^{56} 1867$ fuhr viermal im Monat ein österreichisches Dampfschiff von Triest nach Alexandria ab und französische und englische Schifffahrtslinien verbanden gleichfalls viermal pro Monat die Hafenstädte Marseille und Southampton mit Alexandria. Ebenso häufig fuhren sie dann von Alexandria aus nach Triest, Marseille oder Southampton. ${ }^{57}$

Der direkte Postweg von Alexandria ins Deutsche Reich führte mit einem österreichischen Postschiff nach Triest (siehe Abbildung 2), von wo aus die österreichische Post die Briefe mit dem Zug über die Alpen nach Deutschland brachte. ${ }^{58}$ In der ersten Hälfte des 19. Jahrhunderts war das deutsche Postwesen noch stark zersplittert, doch ab dem 1. Juli 1850 bestand der DeutschÖsterreichische Postverein, dem auch Sachsen beitrat. Ab 1846/47 war Gotha bereits durch die Thüringische Eisenbahn direkt an das deutsche Eisenbahnnetz angeschlossen. ${ }^{59}$ Mit der Gründung des Norddeutschen Bundes 1866 und des Deutschen Reichs 1871 erfolgten weitere Schritte zur Vereinheitlichung des Postwesens.

Eine alternative Postverbindung existierte über England. Alle Briefe, die mit einem englischen Postschiff nach Southampton kamen, wurden zunächst nach

53 Für 1853: Werner Munzinger: Ostafrikanische Studien, Schaffhausen 1864, S. 89-113; für 1861: Heuglin, Reise, S. 27-48; für 1868: Schweinfurth, Im Herzen von Afrika, Bd. 1, S. 7-11.

54 Sammlung Perthes: SPK-10-IV.D C-16: Hermann Berghaus, Allgemeine Welt-Karte in Mercator-Projection zur Übersicht der Postschiffahrt und neuerer Reisen um die Erde, Gotha 1864.

55 Sammlung Perthes: SPK-10-IV.D C-15: Carte des Services à Vapeur dans la Mer des Indes et le Grand Océan. Publié au Dépôt des Cartes de la Marine, 1867.

56 Berghaus, Weltkarte 1864.

57 Sammlung Perthes: SPK-10-IV.D C-15: Carte des Services à Vapeur dans la Mer des Indes et le Grand Océan. Publié au Dépôt des Cartes de la Marine, 1867.

58 Sammlung Perthes: SPA ARCH PGM 62, Bl. 1118: Umschlag eines Briefs von 1862, den Kremer von Kairo via Triest an Peterman in Gotha schickte.

59 Manfred Schäfer: Zur Postgeschichte in Thüringen 1815 bis 1871 (Beiträge zur postgeschichtlichen Forschung, Heft 6), Suhl 1988, S. 24, 34, 50. 
London transportiert. Von dort gelangten sie über die Route Ostende-LüttichAachen-Köln ins Verteilernetz der deutschen Post. ${ }^{60}$

Mit der Eröffnung des Suezkanals 1869 verdichtete sich der Postverkehr im Roten Meer. Ab diesem Jahr konnten Briefe von Massaua nach Aden gebracht werden, von wo aus sie ein Schiff einer englischen, französischen oder niederländischen Postdampfschifflinie direkt nach Europa mitnahm. ${ }^{61}$ Nach einer Übersicht der »Dampfschiffslinien im Weltpostverkehr« von 1883 verliefen nun auch zwei deutsche Schifffahrtslinien von Hamburg nach Shanghai bzw. Sydney, doch diese legten keinen Halt im Roten Meer ein. Sie müssen somit die Post aus den dortigen Häfen in Suez oder Port Said aufgenommen haben. ${ }^{62}$

Ab der Mitte des 19. Jahrhunderts erfolgte schließlich der Aufbau eines Telegraphensystems, das die weltweite Kommunikation erhebliche beschleunigte. ${ }^{63}$ Nach dem Ausbau eines Überlandsystems begannen die TelegraphieGesellschaften ab 1865 mit der Verlegung von Seekabeln, ${ }^{64}$ durch welche auch Afrika an das europäische Telegraphennetz angeschlossen wurde. 1870 entstand die Telegraphenverbindung von Paris nach Algier und in den 1870er Jahren wurde eine Verbindung von Italien nach Alexandrien eingerichtet. ${ }^{65}$ Von Suez aus führte 1874 bereits ein Unterseekabel durch das Rote Meer nach Aden. ${ }^{66}$ Mitte der 1880er Jahre verband auch ein Unterseekabel Triest mit Alexandria und es gab eine Verbindung von Suez nach Suakin. ${ }^{67}$ Dagegen war das Innere Afrikas - abgesehen von der Telegraphenlinie, die Khartum direkt

60 Hans-Jürgen Neumann: Europa und England - 500 Jahre Postgeschichte. Historische Entwicklungsaspekte der Post und Wirtschaft vom 15. bis zum 19. Jahrhundert, Schwalmtal o.D. 2016, S. 369.

61 Sammlung Perthes: SPK-10-IV.D C-16: Übersicht der überseeischen Postdampfschiffslinien im Weltpostverkehr unter Berücksichtigung der Postverbindungen nach den aussereuropäischen Deutschen Consulatsorten. Nach dem Stande am 1. Januar 1883. Bearbeitet im Kursbureau des Deutschen Reichs-Postamts. Zum nur partiellen Ausbau des weltweiten Dampfschifffahrtsverkehrs im 19. Jahrhundert siehe allgemein: Ralf Roth: Verkehrsrevolutionen, in: Reinhard Sieder, Ernst Langthaler (Hrsg.): Globalgeschichte 1800 - 2010, Wien/Köln/Weimar 2010, S. 471-501.

62 Sammlung Perthes: SPK-10-IV.D C-16: Übersicht der überseeischen Postdampfschiffslinien im Weltpostverkehr unter Berücksichtigung der Postverbindungen nach den aussereuropäischen Deutschen Consulatsorten. Nach dem Stand am 1. Januar 1883, bearbeitet im Kursbureau des Deutschen Reichs-Postamts.

63 Huber, Reseau, S. 490-520; S. Roth, Verkehrsrevolution, S. 484.

64 Huber, Reseau, S. 493.

65 Huber, Reseau, S. 506; Sammlung Perthes: SPK-10-IV.D C-09: Welt-Telegraphen-Karte. Herausgegeben vom k. k. Handelsministerium. Wien 1875.

66 Sammlung Perthes: SPK-10-IV.D C-15: Teilkarte der Carte des Communications Télégraphiques internationales, dressée d'après des documents offciels par le Bureau International des Administrations Télégraphiques, Berne 1874.

67 Sammlung Perthes: SPK-10-IV.D C-17: Übersicht der internationalen Telegraphen-Verbindungen mit einer Übersicht der Zeitunterschiede zwischen wichtigeren Orten des Weltverkehrs. Bearbeitet im Telegraphenbetriebs-Bureau des Reichs-Postamts, Berlin 1886. 
mit Kairo verband - bis in die 1880er Jahre und noch lange Zeit darüber hinaus nicht direkt mit diesem globalen Kommunikationsnetz verbunden. ${ }^{68}$

\section{Das Verhältnis von Raum und Zeit}

Eine Auswertung von 109 Briefen, die Georg Rohlfs, Theodor von Heuglin, Werner Munzinger, Georg Schweinfurth und Emin Pascha zwischen 1856 und 1880 aus unterschiedlichen Orten in Äthiopien und im Sudan nach Gotha schickten, liefert Aufschlüsse über die Brieflaufzeiten innerhalb Afrikas und dann innerhalb des europäischen Postsystems nach Gotha. ${ }^{69}$

Während des gesamten Zeitraums gelangten Briefe von Alexandria aus in nicht einmal zwei Wochen nach Gotha. Das entsprach ungefähr den elf Tagen, die 1866-1869 Briefe auch von Alexandria nach London benötigten. ${ }^{70}$ Mögliche Schwankungen in der Transportzeit der hier ausgewerteten Briefe lassen sich dadurch erklären, dass nicht immer sofort ein Postdampfschiff in einen europäischen Hafen abfuhr. Von Kairo aus brauchte ein Brief anfangs etwas länger als von Alexandria aus, aber von 1861 bis 1880 ist eine konstante Beschleunigung des Briefverkehrs zu konstatieren.

Ein Brief, der in Tripolis abgeschickt wurde, benötigte dagegen 1861-1868 noch deutlich länger. Aber zehn Jahre später lässt die Brieflaufzeit von nur elf Tagen darauf schließen, dass nun auch dieser Mittelmeerhafen direkt an das europäische Kommunikationsnetz eingebunden war. Ähnliches galt für die am Roten Meer gelegene Hafenstadt Massaua. Die erhebliche Beschleunigung der Brieflaufzeit von der Mitte der 1860er Jahre bis Mitte der 1870er Jahre dürfte ein Ergebnis der Eröffnung des Suezkanals gewesen sein.

68 Roth, Verkehrsrevolutionen, S. 484.

69 Sammlung Perthes: SPA ARCH PGM 16 (Gerhard Rohlfs), SPA ARCH PGM 62 (Theodor von Heuglin), SPA ARCH PGM 72 (Werner Munzinger), SPA ARCH PGM 354 (Georg Schweinfurth, SPA ARCH PGM 6127/2 (Emin Pascha).

70 Jorma Ahvenainen: The Role of Telegraphs in the 19th Century Revolution of Communications, in: Michael North (Hrsg.): Kommunikationsrevolutionen. Die neuen Medien des 16. und 19. Jahrhunderts, 2. Auflage, Köln, Wien, Weimar 2001, S. 73-80, hier S. 75. 
Tabelle 1: Laufzeit von Briefen aus verschiedenen afrikanischen Orten nach Gotha

\begin{tabular}{|c|c|c|c|c|c|c|c|}
\hline \multirow[t]{2}{*}{ Jahr } & \multirow{2}{*}{$\begin{array}{l}\text { Ort der Ab- } \\
\text { sendung des } \\
\text { Briefs }\end{array}$} & \multicolumn{2}{|c|}{ Brieflaufzeit } & \multirow[t]{2}{*}{ Jahr } & \multirow{2}{*}{$\begin{array}{l}\text { Ort der } \\
\text { Absendung } \\
\text { des Briefs }\end{array}$} & \multicolumn{2}{|c|}{ Brieflaufzeit } \\
\hline & & Monate & Tage & & & Monate & Tage \\
\hline 1857 & Triest & - & 4 & 1880 & Suez & 1 & 10 \\
\hline 1861 & Alexandria & - & $9-11$ & 1857 & Aden & 2 & - \\
\hline $1866 / 68$ & Alexandria & & $10-11$ & 1862 & Djedda & - & 23 \\
\hline 1875 & Alexandria & & 15 & 1865 & Suakin & $31 / 2$ & - \\
\hline $1857 / 58$ & Kairo & - & $21-24$ & $1857-64$ & Massaua & $2-1 \frac{1 / 2}{2}$ & - \\
\hline 1861 & Kairo & - & $9-26$ & 1874 & Massaua & - & 20 \\
\hline $1876-79$ & Kairo & - & $8-14$ & 1865 & Kassala $^{71}$ & $2 \frac{1}{2}$ & - \\
\hline 1880 & Kairo & - & $6-11$ & 1873 & Kassala & 1 & - \\
\hline $1868 / 69$ & Siut $^{72}$ & - & 15 & 1861 & Keren $^{73}$ & $2-3$ & - \\
\hline 1862 & Khartum & 1 & 10 & 1862 & Mai Scheka $^{74}$ & $31 / 2$ & - \\
\hline 1864 & Khartum & 2 & - & 1862 & Adua $^{75}$ & $21 / 2-5$ & - \\
\hline 1869 & Khartum & 1 & 10 & 1862 & Gondar $^{76}$ & $3 \frac{1 / 2-4}{2}$ & - \\
\hline 1862 & El Obeid $^{77}$ & $2-2 \frac{1}{2}$ & - & $1868 / 69$ & Tripolis & - & $18-27$ \\
\hline $1876-78$ & Lado $^{78}$ & $2-2 \frac{1}{2}$ & - & 1878 & Tripolis & - & 11 \\
\hline $1876-78$ & Faschoda $^{79}$ & $21 / 2$ & - & 1865 & Rhadames & 2 & 10 \\
\hline 1869 & Seriba Ghatta ${ }^{80}$ & $51 / 2$ & - & 1865 & Mursuk & 1 & 20 \\
\hline $1876-78$ & Mruli $^{81}$ & $21 / 2$ & - & 1865 & $\begin{array}{l}\text { Schimmedra } \\
\text { in Kaunar }\end{array}$ & 2 & 20 \\
\hline
\end{tabular}

71 Stadt im heutigen Eritrea.

72 Heute Assiut; am Nil gelegene Stadt in Mittelägypten.

73 Stadt im heutigen Eritrea.

74 In den 1860er Jahren Ort an der Grenze zwischen Nordäthiopien und dem südöstlichen Sudan. 75 Stadt in Nordäthiopien.

76 Stadt in Nordäthiopien.

77 Hauptstadt der westsudanesischen Provinz Kordofan.

78 Stadt im Südsudan, nahe der heutigen Haupststadt Juba. In den 1870er und 1880er Jahren Hauptstadt der Provinz Äquatoria.

79 Stadt im Südsudan; existiert heute nicht mehr.

80 Elfenbein- und Sklavenjägerstation im Süden des Sudan, südwestlich der Stadt Wau gelegen; Karte der Entdeckungen Dr. Schweinfurth's in Central-Africa 1874. Karte nach dem Stande der heutigen Kenntnis 1917, in: Georg Schweinfurth: Im Herzen von Afrika. Reisen und Entdeckungen im zentralen Äquatorial-Afrika während der Jahre 1868-1871. Ein Beitrag zur Entdeckungsgeschichte von Afrika, 3. Auflage, Leipzig 1918.

81 In den 1870er/80er Jahren Hauptstadt des Königreichs Buganda; heute in Uganda.

82 Ort in der Sahara, nördlich der Oase Agadem (heute im Niger) gelegen; Gerhard Rohlfs: Quer durch Afrika. Reise vom Mittelmeer nach dem Tschad-See und zum Golf von Guinea, Bd. 1, Leipzig 1874, Karte II: Von Mursuk nach Kuka. 
Deutlich länger dauerte der Transport jedoch dann, wenn die Briefe aus dem Innern des Kontinents zunächst in die Hafenstädte am Mittelmeer oder am Roten Meer gebracht wurden. Die Absender mussten damit rechnen, dass ihre Briefe mindestens anderthalb Monate unterwegs waren. Die sehr kurze Zeit von einem Monat, die 1873 ein Brief von Kassala nach Gotha benötigte, lässt sich dadurch erklären, dass wahrscheinlich noch am selben Tag eine Karawane aus Kassala nach Massaua abging, und dass bei Ankunft der Karawane am Roten Meer ein Postschiff den Brief sofort von Massaua nach Europa transportierte. Ansonsten lag die durchschnittliche Brieflaufzeit bei zwei bis drei Monaten, konnte aber auch sehr viel mehr betragen.

Der Grund für diese enorme Verlängerung der Brieflaufzeit lag im langsamen Transport innerhalb Afrikas, wie die beiden folgenden Beispiele zeigen: Ein Brief, den Georg Schweinfurth am 20. Juli 1869 aus der Seriba des Elfenbeinhändlers Ghatta im Süden des Sudan abschickte, brauchte drei Monate, bis er nach Khartum gelangte (siehe Abbildung 4). Dorthin dürfte er mit einem Elfenbeintransport gelangt sein. Zunächst wurde der Brief über Land bis zum Fluss Djūr Ssuēh gebracht, und dann ging es mit einem Boot den Djūr Ssuēh, den Bahr-el-Ghazal und schließlich den Weißen Nil hinunter bis nach Khartum. Von dort aus erreichte der Brief innerhalb von zweieinhalb Monaten Gotha. ${ }^{83}$ Ebenso wurde ein Brief, den Werner Munzinger am 1. Juni 1867 in Massaua abschickte, am 19. Juli 1867 in Alexandria noch einmal abgestempelt. Am 14. August 1867 erhielt ihn dann August Petermann in Gotha. ${ }^{84}$

Der Grund für die deutlich längere Transportdauer innerhalb Afrikas ist, dass die Briefe nicht innerhalb eines eigenständigen Postsystems, sondern im Rahmen des Karawanensystems transportiert wurden ${ }^{85}$ Doch bei weitem nicht jeden Tag kam eine Karawane durch den Ort. Oftmals sammelten sich auch Karawanen an einem bestimmten Ort, und erst, wenn genügend Händler zusammen waren, damit die Reise lukrativ und sicher genug war, machten sie sich auf den Weg. Solange musste auch der Brief auf seinen Transport warten. In der Zeit bis zur Abreise der Karawane konnten noch weitere Briefe geschrieben werden, oder die Briefe sammelten sich in einem Briefverteilzentrum, bis sie mit einem Postschiff weitertransportiert wurden. So erhielt August Petermann in Gotha am 15. Dezember 1861 insgesamt fünf Briefe, die Theodor von Heuglin ihm in der Zeit vom 3. September bis zum 15. Oktober 1861 aus

83 Sammlung Perthes: SPA ARCH PGM 354, Bl. 123; Karte der Entdeckungen Dr. Schweinfurth's, Karte.

84 Sammlung Perthes: SPA ARCH PGM 72, Bl. 160.

85 Zum Karawanensystem siehe am Beispiel Ostafrikas Michael Pesek: Koloniale Herrschaft in Deutsch-Ostafrika. Expeditionen, Militär und Verwaltung seit 1880, Frankfurt am Main usw. 2005, S. 58-101; Stephen J. Rockel: Decentering Exploration in East Africa, in: Dane Kennedy (Hrsg.): Reinterpreting Exploration. The West in the World, Oxford usw. 2014, S. 172-194. 
Keren zugeschickt hatte. ${ }^{86}$ Ebenso mussten die Reisenden auf ihre Briefe warten, bis eine der Karawanen ihnen ihre Post mitbrachte, wie die Erfahrungen Gustav Nachtigals zeigen.

Die Briefe wurden also innerhalb zweier unterschiedlicher Kommunikationssysteme transportiert. Während innerhalb Afrikas der Transport von Informationen und Gütern auf der Grundlage des bereits lange existierenden Karawanensystems erfolgte, bildeten sich in Europa vom 16. bis zum 19. Jahrhundert eigenständige Kommunikationssysteme aus, die einen konstanten Informationsfluss ermöglichten. Über die hier behandelte Privat- und Geschäftskorrespondenz hinaus zählte hierzu auch ein umfangreiches Medienwesen und ein stark ausgebautes System des Gütertransports. ${ }^{87}$

Diese beiden in Europa und Afrika existierenden Kommunikationssysteme unterschieden sich zunächst also durch ihren Organisationsgrad und ihre Informationsdichte. Im 19. Jahrhundert wurde nach aktuellen Theorien jedoch der Faktor Zeit, das heißt die Geschwindigkeit der Informationsübermittlung, zum bedeutenden Distinktionsfaktor. Schon vor dem 19. Jahrhundert wurden in Europa technische Möglichkeiten entwickelt, die einen schnelleren Transport von Waren und Informationen erlaubten, und diese Beschleunigung verstärkte sich mit der Industriellen Revolution erheblich. ${ }^{88}$ Sinnfälligstes Beispiel ist der Ausbau eines weltumspannenden Telegraphennetzes seit der Mitte des 19. Jahrhunderts, durch den die Übermittlung einer Nachricht von Alexandria nach London 1870 nur noch zwei Tage dauerte. ${ }^{89}$ Doch hierdurch begann sich nun die Zeit vom Raum zu lösen. ${ }^{90}$ Diese Entwicklung setzte sich bis zur digitalen Informationsvermittlung so weit fort, dass wir heute Nachrichten aus der ganzen Welt nahezu in Echtzeit erhalten können.

Nach Roland Wenzlhuemer war diese Beschleunigung ein Motor der Globalisierung. ${ }^{91}$ Auf der Grundlage eines $»$ relativistischen Raumkonzepts $«{ }^{92}$ geht er davon aus, dass der geographische Raum nur eine von mehreren möglichen

86 Sammlung Perthes: SPA ARCH PGM 62, Bl. 1069-1109.

87 Roth, Verkehrsrevolutionen; Requate, Kommunikationsmedien; Hermann Kellenbenz: Technik und Wirtschaft im Zeitalter der Wissenschaftlichen Revolution, in: Carlo M. Cipolla, Knut Borchardt (Hrsg.): Europäische Wirtschaftsgeschichte, Bd. 2: Sechzehntes und Siebzehntes Jahrhundert, Stuttgart/New York 1983, S. 113-169, hier S. 141-145.

88 Kellenbenz, Technik; R. M. Hartwell: Die Dienstleistungsrevolution: die Expansion des Dienstleistungssektors in der modernen Volkswirtschaft, in: Carlo M. Cipolla, Knut Borchardt (Hrsg.): Europäische Wirtschaftsgeschichte, Bd. 3: Die Industrielle Revolution, Stuttgart/New York 1985, S. 233-260, hier S. 246; Reinhart Koselleck: Zeitschichten. Studien zur Historik, Frankfurt am Main 2000, S. 157-161.

89 Ahvenainen, Role, S. 75.

90 Rosa, Beschleunigung, S. 163-165.

91 Wenzlhuemer, Globalization.

92 Hierzu siehe Martina Löw: Raumsoziologie, Frankfurt am Main 2001, S. 27-28. 
Varianten des Raums ist. Zu jeder möglichen Art von Interaktion gehöre ein eigener Raum. ${ }^{93}$ Die im Rahmen der Globalisierung erfolgenden Veränderungen weltweiter Interaktion ließen Räume entstehen, die neue Formen der Verbindung und Interaktion zwischen Objekten in Räumen repräsentierten. Es entstanden Räume von Zeitstrukturen, in die nur jene Weltregionen integriert waren, die an diesen Kommunikationsformen teilhatten. Dagegen blieben andere Weltregionen wie das Innere Afrikas davon ausgeschlossen. ${ }^{94}$

Doch solche neuen Raumstrukturen entwickelten sich in Europa schon vor dem Ausbau des globalen Telegraphennetzwerks. Der Aufbau eines Postsystems seit dem 16. Jahrhundert führte zur allmählichen Entwicklung eigenständiger Infrastrukturen, ${ }^{95}$ welche bis zum 18. Jahrhundert die kontinuierliche Übermittlung von Briefen garantierten, und die hier vorgelegten Brieflaufzeiten belegen auch eine größere Transportgeschwindigkeit. Für die Entwicklung eines globalen Transport- und Informationsnetzwerks im 19. Jahrhundert wird der frühneuzeitliche transatlantische Dreieckshandel zwischen Europa, Afrika und Amerika ${ }^{96}$ ebenso wichtig gewesen sein, wie die Weltumsegelungen seit dem 18. Jahrhundert. Diese Entwicklung fand ihren Niederschlag auch in Wissenschaft und Kultur. So trugen die mit Antoine de Bougainvilles und James Cook um die Welt reisenden Naturwissenschaftler auch dazu bei, die Pflanzen und Tiere unter einer einheitlichen, weltumspannenden Nomenklatur zu erfassen. ${ }^{97}$ Dieses weltumspannende Transport- und Informationsnetzwerk fand seinen bildlich-kartographischen Ausdruck in der Chart of the World, einer ab 1863 vom Verlag Justus Perthes veröffentlichten Weltkarte, in der alle Schifffahrtslinien auf den Weltmeeren eingezeichnet sind. ${ }^{98}$

Die hier behandelten Briefe wurden teilweise innerhalb dieses weltumfassenden Kommunikationsnetzwerks transportiert, aber eben nicht vollständig. Die oben angeführten Brieflaufzeiten belegen deutliche Differenzen zwischen europäischen und innerafrikanischen Kommunikationssystemen. Diese zeitlichen Differenzen wurden durch strukturelle Unterschiede bedingt: Dem im 19. Jahrhundert gut ausgebauten weltumfassenden Kommunikationssystem standen in Afrika ganz anders geartete Strukturen gegenüber. Diese orientierten sich nicht an den Bedürfnissen des Postverkehrs, und damit folgte der Brief-

93 Wenzlhuemer, Globalization, S. 27.

94 Wenzlhuemer, Globalization, S. 29-31.

95 Vgl. Wolfgang Behringer: Im Zeichen des Merkur. Reichspost und Kommunikationsrevolution in der Frühen Neuzeit, Göttingen 2003; Neumann, Europa und England.

96 Albert Wirz: Sklaverei und kapitalistisches Weltsystem, Frankfurt am Main 1984.

97 Jean Marc Drouin: Von Linné zu Darwin, in: Michel Serres (Hrsg.): Elemente einer Geschichte der Wissenschaften, 2. Auflage Frankfurt am Main 2002, S. 569-595.

98 Iris Schröder: Eine Weltkarte aus der Provinz. Die Gothaer Chart of the World und die Karriere eines globalen Bestsellers, in: Historische Anthropologie 25 (2017), S. 353-376. 
verkehr im Innern Afrikas nicht einem eigenen zeitlichen Rhythmus, sondern musste sich den Rhythmen des Karawanenhandels unterwerfen.

Die hier dargestellte Briefkommunikation bewegte sich in beiden Raumund Zeitstrukturen. Auf diese Unterschiede mussten sich die in Äthiopien und im Sudan reisenden Europäer einstellen, sie mussten nicht nur damit rechnen, dass ihre Briefe lange unterwegs waren, sondern sie mussten sich auch darauf einstellen, dass Sendungen verloren gingen. Daher war es üblich, dass die Reisenden in ihren Briefen zuvor bereits abgesandte Briefe erwähnten, und von erhaltenen Postsendungen berichteten. Mit ihrer Korrespondenz beteiligten sich die europäischen Reisenden jedoch am Aufbau eines eigenen Kommunikationsraums über kulturelle Grenzen hinweg, in dem sie einen Informationsaustausch zwischen europäischer Gelehrsamkeit und afrikanischen Gesprächspartnern begründeten. Dessen besondere Ausformungen sind in der Zukunft noch weiter zu untersuchen.

\section{Literatur- und Quellenverzeichnis}

Jorma Ahvenainen: The Role of Telegraphs in the 19th Century Revolution of Communications, in: Michael North (Hrsg.): Kommunikationsrevolutionen. Die neuen Medien des 16. und 19. Jahrhunderts, 2. Auflage, Köln, Wien, Weimar 2001, S. 73-80.

Mario J. Azevedo: Roots of Violence. A History of War in Chad, Amsterdam 1998.

Heinrich Barth: Reisen in Nord- und Central-Africa in den Jahren 1859 bis 1855, Bd. 5, Gotha 1858.

Wolfgang Behringer: Im Zeichen des Merkur. Reichspost und Kommunikationsrevolution in der Frühen Neuzeit, Göttingen 2003.

M. W. Daly: Darfur's Sorrow. A History of Destruction and Genocide, Cambridge usw. 2007.

Felix Driver, Lowri Jones: Hidden Histories of Exploration. Researching the RGS-IGB Collections, London 2009.

Jean Marc Drouin: Von Linné zu Darwin, in: Michel Serres (Hrsg.): Elemente einer Geschichte der Wissenschaften, 2. Auflage Frankfurt am Main 2002, S. 569-595.

Colette Dubois: Morphologies de Khartoum: conflits d'identité (1820 - début du XXe siècle), in: Hervé Bleuchot, Christian Delmet, Derek Hopwood (Hrsg.): Sūdān. History, identitiy, ideology. Histoire, identités, idéologies, Aix-en-Provence/Oxford 1991, S. 13-32.

Ute Frevert, Wolfgang Braungart (Hrsg.): Sprachen des Politischen. Medien und Medialität in der Geschichte, Göttingen 2004.

Wilhelm Götze: Die Verkehrswege im Dienste des Welthandels. Eine historisch-geographische Untersuchung, Stuttgart 1888.

R. M. Hartwell: Die Dienstleistungsrevolution: die Expansion des Dienstleistungssektors in der modernen Volkswirtschaft, in: Carlo M. Cipolla, Knut Borchardt (Hrsg.): Europäische Wirtschaftsgeschichte, Bd. 3: Die Industrielle Revolution, Stuttgart/New York 1985, S. 233-260. 
Dietmar Henze: Enzyklopädie der Entdecker und Erforscher der Erde, Bd. 5, Neuausgabe Darmstadt 2011.

Theodor von Heuglin: Reise nach Abessinien, den Gala=Ländern, Ost=Sudán und Chartúm in den Jahren 1861 und 1862, Jena 1868.

William Huber: Le réseau télégraphique du globe, in: Bulletin de la Société de Géographie $1873 / 2$, S. 490-520.

Hermann Kellenbenz: Technik und Wirtschaft im Zeitalter der Wissenschaftlichen Revolution, in: Carlo M. Cipolla, Knut Borchardt (Hrsg.): Europäische Wirtschaftsgeschichte, Bd. 2: Sechzehntes und Siebzehntes Jahrhundert, Stuttgart/New York 1983, S. 113-169.

Dane Kennedy: The Last Blank Spaces. Exploring Africa and Australia, Cambridge, Mass./ London 2013.

Christian Kirchen: Emin Pascha. Arzt - Abenteurer - Afrikaforscher, Paderborn 2014.

Habbo Knoch, Daniel Morat: Medienwandel und Gesellschaftsbilder 1880-1960. Zur historischen Kommunikologie der massenmedialen Sattelzeit, in: Ders., Ders. (Hrsg.): Kommunikation als Beobachtung. Medienwandel und Gesellschaftsbilder 1880-1960, München 2002, S. 9-33.

Reinhart Koselleck: Zeitschichten. Studien zur Historik, Frankfurt am Main 2000.

Benjamin N. Lawrance, Emily Lynn Osborn, Richard L. Roberts (Hrsg.): Intermediaries, Interpreters, and Clerks. African Employees in the Making of Colonial Africa, Madison, Wisconsin 2006.

Martina Löw: Raumsoziologie, Frankfurt am Main 2001.

Ernst Marno: Ueber die Pflanzenbarren im oberen Weißen Nil, in: Mittheilungen der Geographischen Gesellschaft in Wien 1880, S. 401-409.

Volker Matthies: Im Schatten der Entdecker. Indigene Begleiter europäischer Forschungsreisender, Bonn 2018.

Armand Mattelart: Kommunikation ohne Grenzen? Geschichte der Ideen und Strategien globaler Vernetzung, 2. Auflage Berlin 2007.

Werner Munzinger: Ostafrikanische Studien, Schaffhausen 1864.

Gustav Nachtigal: Saharâ und Sûdân. Ergebnisse Sechsjähriger Reisen in Afrika, Bd. 3, Leipzig 1889.

Hans-Jürgen Neumann: Europa und England - 500 Jahre Postgeschichte. Historische Entwicklungsaspekte der Post und Wirtschaft vom 15. bis zum 19. Jahrhundert, Schwalmtal 2016.

R. S. O'Fahey: The Darfur Sultanate. A History, London 2008.

Ohne Verfasser: Werner Munzinger's Bericht über seine und G. Th. Kinzelbach's Reise nach El Obed 1862, in: Mittheilungen aus Justus Perthes Geographischer Anstalt über wichtige neue Erforschungen auf dem Gesamtgebiete der Geographie, Bd. 9 (1863), S. 183-190.

Ohne Verfasser: Ein Brief von Th. Kinzelbach aus El Obed, der Hauptstadt von Kordofan, in: Mittheilungen aus Justus Perthes Geographischer Anstalt über wichtige neue Erforschungen auf dem Gesamtgebiete der Geographie, Bd. 9 (1863), S. 217-225.

Ohne Verfasser: Moriz v. Beurmann's Tod nebst Übersicht seiner Reise (1861-1863) so wie derjenigen von Overweg (1850-1852), Vogel (1853-1856) und Steudner (1861-1863), in: Mittheilungen aus Justus Perthes Geographischer Anstalt über wichtige neue Erforschungen auf dem Gesamtgebiete der Geographie, Bd. 10 (1864), S. 25-30. 
Ohne Verfasser »Nachrichten über Ed. Vogel's Schicksal von der Munzinger'schen Expedition «, in: Mittheilungen aus Justus Perthes Geographischer Anstalt über wichtige neue Erforschungen auf dem Gesamtgebiete der Geographie, Bd. 8 (1862), S. 346-350.

Michael Pesek: Koloniale Herrschaft in Deutsch-Ostafrika. Expeditionen, Militär und Verwaltung seit 1880, Frankfurt am Main usw. 2005.

August Petermann: Die Expedition nach Central Africa II. Dr. H. Barths Rückreise von Timbuktu nach Kano, vom 8. Juli bis 17. Oktober 1854, in: Mittheilungen aus Justus Perthes Geographischer Anstalt über wichtige neue Erforschungen auf dem Gesamtgebiete der Geographie, Bd. 1 (1855), S. 85-89.

Reiner Prass: Forschungsreise und Wissensproduktion in Afrika in der Mitte der 19. Jahrhunderts, in: Themenportal Europäische Geschichte 2019, URL: https://www.europa.clioonline.de/essay/id/fdae-1728.

Jörg Requate: »Kommunikationsmedien und Gesellschaft«, in: Reinhard Sieder/Ernst Langthaler (Hrsg.): Globalgeschichte 1800-2010, Wien/Köln/Weimar 2010, S. 439-469.

Stephen J. Rockel: Decentering Exploration in East Africa, in: Dane Kennedy (Hrsg.): Reinterpreting Exploration. The West in the World, Oxford usw. 2014, S. 172-194.

Gerhard Rohlfs: Quer durch Afrika. Reise vom Mittelmeer nach dem Tschad-See und zum Golf von Guinea, Bd. 1, Leipzig 1874.

Hartmut Rosa: Beschleunigung. Die Veränderung der Zeitstruktur in der Moderne, Frankfurt am Main 2005.

Ralf Roth: Verkehrsrevolutionen, in: Reinhard Sieder/Ernst Langthaler (Hrsg.): Globalgeschichte 1800 - 2010, Wien/Köln/Weimar 2010, S. 471-501.

Manfred Schäfer: Zur Postgeschichte in Thüringen 1815 bis 1871 (Beiträge zur postgeschichtlichen Forschung, Heft 6), Suhl 1988.

Iris Schröder: Eine Weltkarte aus der Provinz. Die Gothaer Chart of the World und die Karriere eines globalen Bestsellers, in: Historische Anthropologie 25 (2017), S. 353-376.

Georg Schweinfurth: Im Herzen von Afrika. Reisen und Entdeckungen im centralen Aequatorial-Afrika während der Jahre 1868 bis 1871, Bd. 2, Leipzig/London 1874.

Georg Schweinfurth: Im Herzen von Afrika. Reisen und Entdeckungen im zentralen Äquatorial-Afrika während der Jahre 1868-1871. Ein Beitrag zur Entdeckungsgeschichte von Afrika, 3. Auflage, Leipzig 1918.

Wolfgang Struck: Karten-Legenden. August Petermanns Verschollene, in: Irina Gradinari, Dorit Müller, Johannes Pause (Hrsg.): Versteckt - Verirrt - Verschollen. Reisen und Nicht-Wissen, Wiesbaden 2016, S. 69-83.

David Vincent: The Rise of Mass Literacy. Reading and Writing in Modern Europe, Cambridge/Oxford/Malden, MA 2000.

Hermann Wagner (Bearb.): Schilderung der Reisen und Entdeckungen des Dr. Eduard Vogel in Central-Africa in der großen Wüste, in den Ländern des Sudan, nach den Originalquellen bearbeitet, Leipzig 1860 .

Petra Weigel: Die Sammlung Perthes Gotha, Forschungsbibliothek Gotha, in: Patrimonia 254, Berlin 2011.

Roland Wenzlhuemer: Globalization, communication and the concept of space in global history, in: Historical Social Research 35 (2010), S. 19-47.

Albert Wirz: Sklaverei und kapitalistisches Weltsystem, Frankfurt am Main 1984. 
Open-Access-Publikation im Sinne der CC-Lizenz BY-NC-ND 4.0 (C) 2021, Vandenhoeck \& Ruprecht GmbH \& Co. KG, Göttingen ISBN Print: 9783847112280 - ISBN E-Lib: 9783737012287 


\section{Abbildungen}

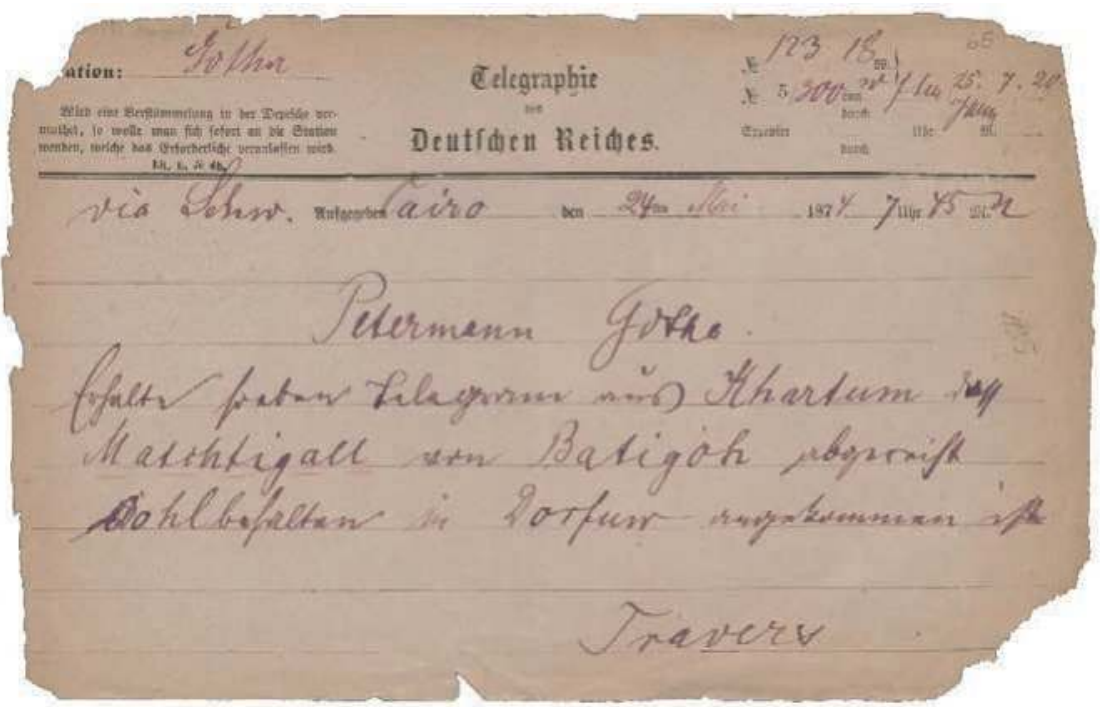

Abbildung 1: Telegramm mit der Nachricht von der Ankunft Gustav Nachtigals in Darfur.



Abbildung 2: Umschlag eines Briefs an Petermann von 1862 mit der Angabe "via Triest«. 


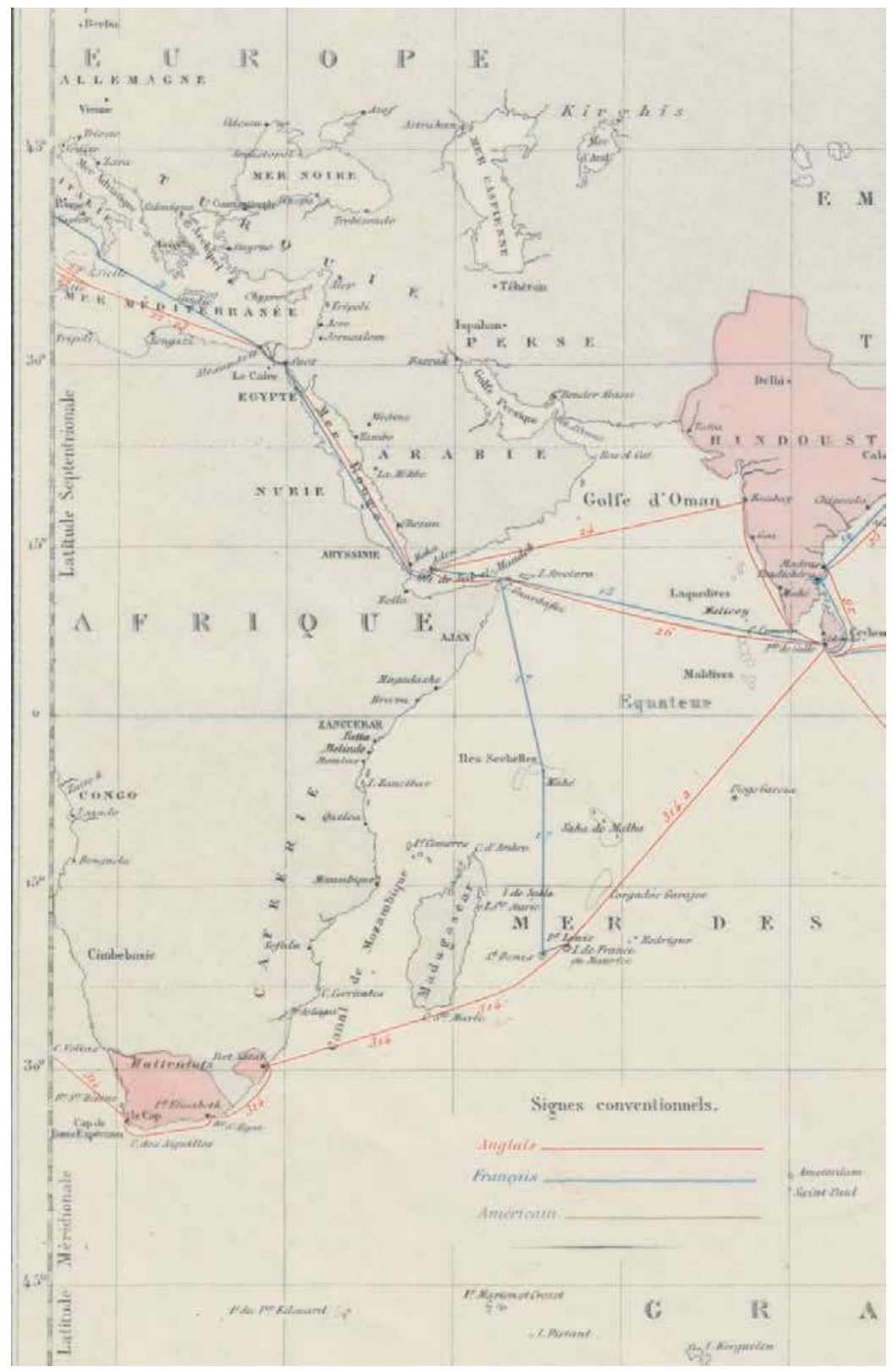

Abbildung 3: Auszug aus Carte des Services à Vapeur dans la Mer des Indes et le Grand Océan (1867) mit den Dampfschiffverbindungen im Roten Meer und im Mittelmeer, verbunden durch eine Eisenbahnlinie von Suez nach Alexandria 


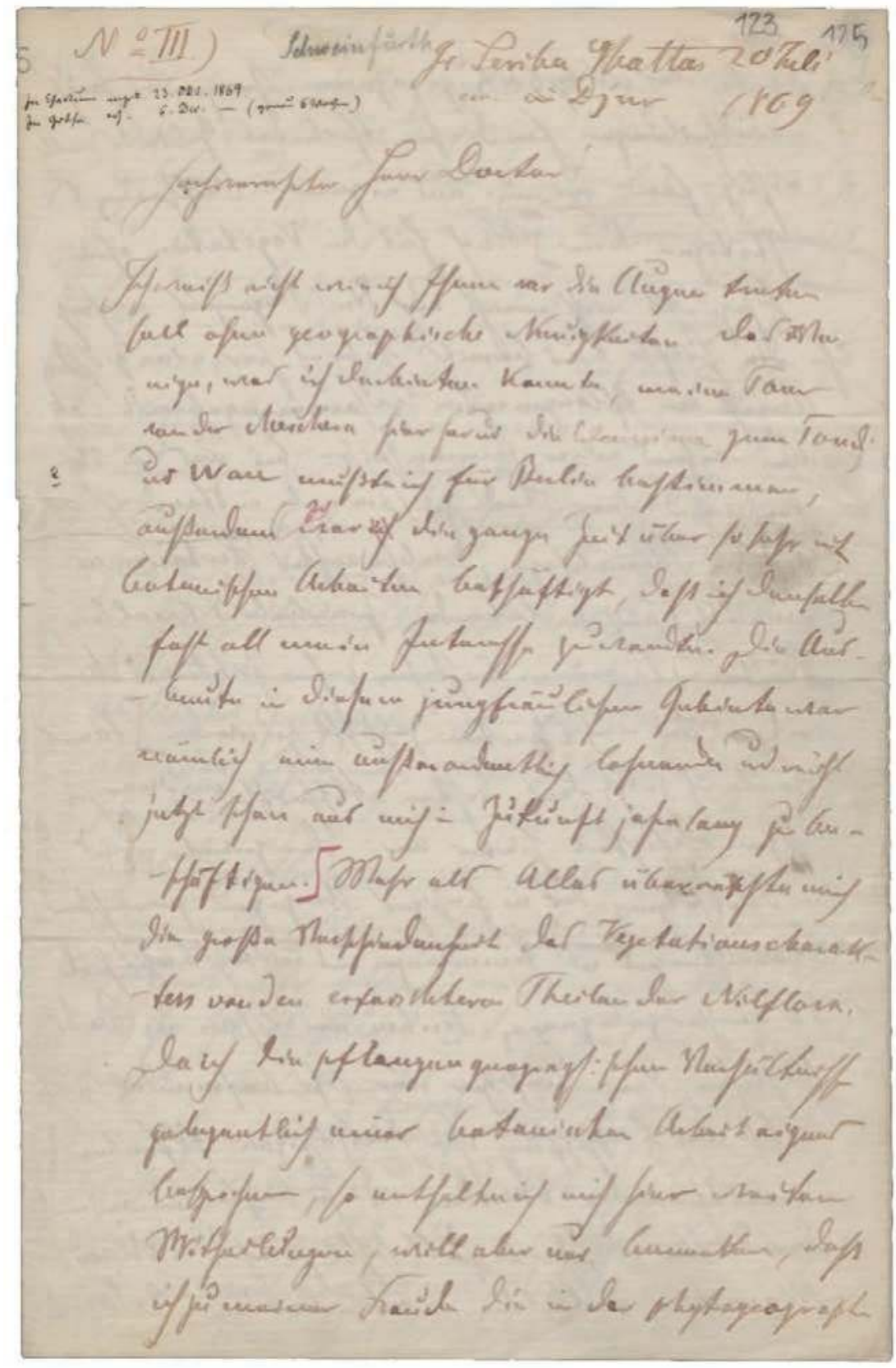

Abbildung 4: Brief Georg Schweinfurths, den er 1869 aus dem heutigen Südsudan an August Petermann sandte. Am oberen Rand sind die Zwischenstationen des Postweges angegeben. 
Open-Access-Publikation im Sinne der CC-Lizenz BY-NC-ND 4.0 (C) 2021, Vandenhoeck \& Ruprecht GmbH \& Co. KG, Göttingen ISBN Print: 9783847112280 - ISBN E-Lib: 9783737012287 


\section{Die Organisation und die Verwaltung der sowjetischen Militär-Feldpost im Zweiten Weltkrieg}

\section{Einleitung}

Die Feldpost erfüllte während des Krieges eine besondere Funktion. Sie war das wichtigste Verbindungsglied zwischen den Militärangehörigen der Roten Armee, die sich an der Front aufhielten, und ihren Verwandten und Freunden, die sich im Hinterland befanden. ${ }^{1}$

Die rechtzeitige Zustellung der Post von der Feldarmee ins Hinterland und in die andere Richtung sollte die Moral der Soldaten und Kommandanten der Sowjetischen Streitkräfte sicherstellen. Der regelmäßige Empfang von Informationen aus der Heimat trug sowohl zu einem Anstieg des Vertrauens in der Rechtmäßigkeit des Militärs als auch zur Stärkung des Glaubens an die Führung des Landes bei. So war die Qualität der Arbeitsleistung der Feldpost auch einer der Faktoren, die die Kampffähigkeit der Roten Armee bestimmten (siehe Abbildung 1).

Außerdem waren die Mitteilungen über den Tod von Soldaten oder Offizieren, die per Post an die Hinterbliebenen versandt wurden, wichtige Dokumente für die sozialen Absicherung der Familien, da sie für die Beantragung von Rentenzahlungen für die Behörden gebraucht wurden. Dies machte die Post, einschließlich der Feldpost, zu einem praktisch alternativlosen Dokumentenmanagementsystem des riesigen Landes (siehe Abbildung 2).

Ziel des Aufsatzes ist es, die wichtigsten Phasen der Entstehung und Entwicklung der sowjetischen Feldpost während des Zweiten Weltkriegs zu beschreiben und die wichtigsten Merkmale der Verwaltung des Militärpostdienstes unter militärischen Bedingungen aufzuzeigen. Besondere Aufmerksamkeit

1 YU.P Agafonov:. Soldatskij treugol'nik. Velikaya Otechestvennaya vojna v sud'bah zaural'cev. SHadrinsk: izd-vo PO «Iset'», 2001; Gluhov A.I. Zapiski polkovogo pochtal'ona. SPb.: »Ivan Fyodorov«, 2005; Zhukov YU.A. Soldatskie dumy: o chyom rasskazyvali pis'ma s frontov Velikoj Otchestvennoj vojny. M.: Sovetskaya Rossiya, 1987. 
soll den Herausforderungen gewidmet werden, mit denen die Führungskräfte des Landes bei der Sicherung der Arbeit der Post in diesem Zeitraum konfrontiert waren.

\section{Die Probleme der Militär-Feldpost zu Beginn des Krieges}

Die Regierung der UdSSR hatte bereits zu Beginn des Kriegs der militärischen Kommunikation im Allgemeinen und der Arbeit des Feldpostdienstes im Besonderen eine erhöhte Aufmerksamkeit geschenkt. Jedoch wurde die Arbeit der Militär-Feldpost durch die Tatsache verkompliziert, dass die Posteinheiten dem Volkskommissariat der Kommunikation (NKS) der UdSSR untergeordnet waren, welches ursprünglich eine zivile und keine militärische Einrichtung war.

Die Kommunikations-Organe der Feldarmee hingegen wurden dem Kommunikationsamt der Roten Armee untergeordnet, das Teil des Volkskommissariats der Verteidigung der UdSSR war. Diese Institution war somit Teil des militärischen Apparates.

Der Leiter des Volkskommissariats der Kommunikation zu Beginn des Krieges war Ivan Terentyevich Peresypkin. ${ }^{2}$ Der Leiter des Kommunikationsamtes der Roten Armee war der Generalmajor der Kommunikationstruppen Nikolay Ivanovich Gapić. ${ }^{3}$ Das Verhältnis zwischen diesen beiden Strukturen wurde in den ersten Monaten des Krieges nicht auf der richtigen Ebene etabliert.

Theoretisch wurde die Abgrenzung der Funktionen der Volkskommissariate für Kommunikation, Verteidigung und Marine in der Verordnung über den Dienst der Feld- und Marinepost der Roten Armee und der Marine der Union der SSR vorgeschrieben. Dieses Dokument wurde am 27. Juni 1941 von dem stellvertretenden Volkskommissar für Verteidigung Armeegeneral G. K. Schukow, von dem stellvertretenden Volkskommissar der Kriegsmarine, Admiral L. M. Haller und vom Volkskommissar der Kommunikation Peresypkin genehmigt. In diesem Dokument wurde festgestellt, dass der Feld- und Marinepostdienst mit der Ankündigung der Mobilisierung "für den Dienst der Postkommunikation während des Krieges der Armeekorps, Schiffe, Verbindungen,

2 Iskusstvo pobezhdat': Polkovodcy Vtoroj Mirovoj vojny. M.: Pashkov dom, 2005; Peresypkin I.T. Voennaya radiosvyaz'. M.: Voenizdat, 1962; Polkovodcy i voenachal'niki Velikoj Otechestvennoj. M.: Molodaya gvardiya, 1985; Vojska svyazi Sovetskoj Armii: Kratkij ocherk. M.: Voenizdat, 1948.

3 Komandnyj i nachal'stvuyushchij sostav Krasnoj Armii v 1940-1941 gg.: Struktura i kadry central'nogo apparata NKO SSSR, voennyh okrugov i obshchevojskovyh armij: Dokumenty i materialy/Pod red. V. N. Kuzelenkova. M.-SPb.: Letnij sad, 2005. 
Mitarbeiter, Institutionen und Einrichtungen der roten Armee und der Marine und des gesamten Personals" geschaffen wurde.

Gemäß der Verordnung wurden Einheiten des NKS in die Struktur von Institutionen des militärischen Büros eingebunden. Infolgedessen sah die vertikale Hierarchie der Führungsgremien folgendermaßen aus:

1. Zentrale militärische Verwaltung des NKS.

2. Front-NKS-Feldkommunikationssteuerung.

3. Die Abteilungen der Feldkommunikation des NKS bei den Hauptquartieren der Armeen.

4. Marine-stationäre Teile der Flotten (Flottilleneinheiten).

Außerdem wurden in der Verordnung vom 27. Juni auch die Institutionen registriert, die für die Arbeit der Militär-Feldpost direkt verantwortlich waren. Dies waren die Militär-Post Sortierstellen, das Militär-Bureau, das den Geldtransfer kontrollierte, die Militärpostämter bei den Hauptquartieren der Feldarmee, Feldpoststationen bei den Hauptquartieren der Frontverbände, Armeen, Korps, Divisionen, einzelnen Brigaden, Luftverbindungen und Versorgungsstationen. Hinzu kamen Marinepostämter der einzelnen Marine Basen, Marinepoststationen für die Verbindungen und Truppenteile, die von den Flottenbasen (Flottillen) entfernt wurden sowie Armeepostboten (Spediteuren) bei Armeekorps, Schiffen und Institutionen der Roten Armee und der Marine.

Die einzelnen Schritte der Herausbildung dieser Institutionen sollten von einer ziemlich engen Koordinierung der militärischen und zivilen Abteilungen begleitet werden. Zum Beispiel mussten sich militärische Postboten durch die Ordnung Ihrer Kommandanten und Chefs von dem Personal der Armeekorps, Schiffe und Einrichtungen abheben. Die Truppe war verpflichtet, die Postboten mit den notwendigen Transportmitteln in den Feld- und Marinepoststationen sowie mit entsprechenden Möglichkeiten für die Aufbewahrung der Korrespondenz zu versorgen. Dabei wurde die Ausbildung für Verwaltungsorgane und Einrichtungen der Feld- und Marinepost während der Friedens- und Kriegszeit dem Volkskommissariat für Kommunikation übertragen. Dieses Kommissariat erarbeitete auch Personalausstattung, Zeugnisse, Vorschriften und Anweisungen und genehmigte diese gemeinsam mit dem Verteidigungsministerium. Für die Besetzung der Organe des Militärpostdienstes durch qualifizierte Arbeiter war auch das NKS verantwortlich, was angesichts der Besonderheiten seiner Arbeit in Friedenszeiten nicht überrascht. Der gesamte Rest des Personals, für das keine spezielle Ausbildung erforderlich war, wurde vom Verteidigungsministerium ernannt. Alle Feld- und Marineposteinrichtungen wurden durch eine gemeinsame Verordnung des Volkskommissariats 
für Verteidigung und des Volkskommissariats der Marine mit Fahrzeugen, Ersatzteilen sowie Wassertransportmitteln und Pferden ausgestattet. Diese waren auch verantwortlich für die Versorgung der Militär-Feldpost mit Lebensmitteln, Futter, Technik, medizinische Versorgung, Veterinärmedizin sowie Transport-, Brenn- und Schmierstoffen. Des weiteren wurden die Einrichtungen der Feld- und Marinepost mit speziellen posttechnischen Gütern, Postzahlungszeichen und Betriebsmaterialien durch das Volkskommissariat für Kommunikation versorgt. Alle Leistungen, die für die Versorgung der Organe der Militär-Feld- und Marinepost während der Kriegszeit notwendig waren, wurden letztlich von zwei militärischen Volkskommissariaten erbracht.

Die Abgrenzung der Befugnisse des Militärs und der Zivilbehörden betraf auch die direkte Verwaltung der Militär-Feldpost. Die operative Leitung der Feld- und Marinepost wurde jeweils von den Kommunikationschefs der Roten Armee und der Marine, den Kommunikationschefs der Fronten, Armeen, Korps, Divisionen, Brigaden, Luftvereinigungen und Militärstraßen sowie den Kommunikationsabteilungen der Flotten durchgeführt. Gleichzeitig wurde die direkte Leitung des Feld -und Marinepostdienstes von Beamten des Volkskommissariats für Kommunikation durchgeführt. ${ }^{4}$

Es muss betont werden, dass alle diese Chefs wiederum operativ den entsprechenden militärischen Kommunikationschefs unterstellt waren. Dieser Umstand hat das Management das Feldpost jedoch freilich nicht übersichtlicher und einfacher gemacht. Die Funktionen ziviler und militärischer Strukturen waren stark miteinander verflochten und schufen somit die Grundlage für vielfältige Redundanzen und Überschneidungen. Dies musste zwangsläufig die Arbeitsqualität des Postdienstes stark beeinträchtigen.

In der Verordnung vom Juni 1941 wurden darüber hinaus auch die verschiedenen Möglichkeiten von Postsendungen, die für den Empfang an die bzw. von der Adresse der Armee und Flotte zugelassen waren und deren individuelle Handhabung aufgelistet. Die Liste umfasste: einfache und individuelle offene und geschlossene Briefe und Pakete, Pakete mit einem Gewicht von bis zu $8 \mathrm{~kg}$, Postanweisungen, Briefe mit einem erklärten Wert von bis zu 500 Rubel - diese wurden jedoch nur an die Adresse der Staatsbank oder von ihr ausgehend akzeptiert - sowie Zeitschriften. Dabei konnte das Gewicht der Pakete, die vom jeweiligen Armeekorps oder Schiff mit den Sachen der neu Mobilisierten oder gefallenen Soldaten geschickt wurden, gegenüber der angegebenen Begrenzungen der Posteinrichtungen auf jeweils $20 \mathrm{~kg}$ erhöht werden.

4 Polozhenie o sluzhbe polevoj i voenno-morskoj pochty. Central'nyj arhiv Ministerstva oborony Rossijskoj Federacii (CAMO RF). F. 219. Op. 679. D. 5. L. 10, online unter:/pamyat-naroda.ru (letzter Zugriff: 14.02.2020). 
Pakete mit Geschenken für Teile der Roten Armee oder der Marine waren überhaupt keinen Beschränkungen unterworfen.

Es sollte beachtet werden, dass alle einfachen Briefe und Postkarten, die von Soldaten und Offizieren der Roten Armee geschickt wurden oder an sie adressiert waren, kostenlos verschickt und zugestellt wurden. Auch die Übersendung des persönlichen Besitzes der Gefallenen war kostenfrei. Den entsprechenden Sendungen wurde stattdessen an den Feldpoststationen ein entsprechendes Zertifikat mit der Unterschrift des befehlshabenden Kommandanten der jeweiligen militärischen Einheit beigefügt. Die übrigen Postsendungen wurden nach den im Volkskommissariat für Kommunikation beschlossenen Tarifen bezahlt.

In der Anfangsphase des Krieges war es nicht einfach, die Arbeit der Militär-Feldpost unter den Bedingungen der Front, die sich schnell nach Osten verlagerte, zügig zu organisieren. Der Militärkorrespondent und Schriftsteller Konstantin Michailowitsch Simonow, ${ }^{5}$ der sich in den ersten Tagen des Krieges an der sowjetischen Westfront in Weißrussland aufhielt, beschrieb die Situation so:

»Unsere Zeitung hat umsonst gearbeitet. Weder von einer Feldpost noch von einem regelmäßigen Zeitungsversand war die Rede. Vierzigtausend Exemplare wurden gedruckt und sie wurden überall transportiert, wo es möglich war, auf zwei oder drei Lastwagen. Und sie kamen in eine, zwei oder drei Divisionen. Und davon, dass sich die Zeitung über die ganze Front zerstreut, konnte in diesen Tagen keine Rede sein ${ }^{6}$.

So führten das Chaos und die Verwirrung der ersten Kriegstage dazu, dass eine effektiv koordinierte Arbeit des Militärpostdienstes und anderer Abteilungen an der Front und in der Etappe nicht zustande kamen.

Im Juli 1941 drückte der Volkskommissar der Verteidigung der UdSSR, Joseph Vissarionowitsch Stalin, seine Unzufriedenheit mit der Arbeit von Gapić aus. Nach einer Sitzung im Kreml am 22. Juli 1941 wurde der Generalmajor von seiner Position »entfernt«. Peresypkin beschrieb dieses Ereignis in seinen

5 Der sowjetische Schriftsteller und Lyriker Simonow, war Kriegsberichterstatter im JapanischSowjetischen Grenzkonflikt (1938/39) im Verlauf des Zweiten Weltkrieges als Offizier der Propagandaeinheiten der Roten Armee - zuletzt im Rang eines Obersten - eingesetzt und gilt als einer der bekanntesten und meistgelesenen Kriegsberichterstatter der UDSSR. Zu Simonow siehe unter anderem Günter Warm: Konstantin Simonow. In: Urania Universum. Jg. 19, 1973, S. 415-422.

6 Konstantin M. Simonov: Raznye dni vojny. Dnevnik pisatelya. M.: Hudozhestvennaya literatura, Moskva 1982, URL: http://militera.lib.ru/db/simonov_km/index.html (letzter Zugriff: 04.10. 2019). In deutscher Ausgabe unter anderem: Konstantin Simonov: Kriegstagebücher, DDRLizenzausgabe, München 1979. 
Memoiren ${ }^{7}$. Im August wurde Gapić verhaftet und bis 1952 in Haft gehalten. 1953, nach dem Tod Stalins, wurde er rehabilitiert. ${ }^{8}$

Peresypkin begann daraufhin, die Positionen des Volkskommissars der Kommunikation und des Leiters des Kommunikationsamtes der Roten Armee zu kombinieren. Außerdem wurde er zudem zum stellvertretenden Volkskommissar der Verteidigung der UdSSR ernannt. Am 28. Juli 1941 wurde das Kommunikationsamt der Roten Armee ins Hauptamt der Kommunikation der Roten Armee umstrukturiert. Im Rahmen dieses Amtes agierte die Dislokations-Postabteilung. Diese Abteilung war vor allem für die Nummerierung von Truppenteilen und Feldpoststationen verantwortlich.

So wurde Ende Juli 1941 das Management der Kommunikation und der militärischen Feldpost in den Händen einer Person konzentriert. Dies erhöhte zwar drastisch das Arbeitsvolumen und den Umfang der Verantwortung Peresypkins, aber übte seine Funktionen sehr energisch und aktiv aus.

In den ersten Monaten des Krieges waren die Hauptbemühungen des neuen stellvertretenden Volkskommissars der Verteidigung darauf ausgerichtet, die Effizienz der Kommunikation in den Streitkräften zu erhöhen. Die Kommunikation war lange Zeit eine der Schwächen der Roten Armee, sodass gerade sie dringend einer Modernisierung bedurfte.

Die Tätigkeit des Militärpostdienstes stieß in der Anfangsphase des Großen Vaterländischen Krieges auch auf eine Reihe von Problemen, die eine dringende Lösung erforderten.

Das erste Problem war organisatorischer Art. Die bestehende Ordnung der Richtung der Postkorrespondenz wurde auch durch die mehrfach erwähnte Verordnung vom 27.Juni 1941 bestimmt. Von den zivilen Unternehmen der Kommunikation des Volkskommissariats für Kommunikation wurden Briefe und andere Arten von Korrespondenz an die Militärpostsortierstellen gemäß dem vom Volkskommissariat geschaffenen Sonderplan übermittelt. In diesen Militärpostsortierstellen mussten alle Postsendungen nach Feld- und Marinepoststationen (Büros) sortiert, in Säcke und >Ledersachen` verschlossen sowie an die Austauschstellen der Militärpostbasen der Armeen, Feldpoststationen der Frontquartiere und anderer Institutionen weitergeleitet werden. Dann sortierten die Militärpoststationen die Post nach den jeweils diesen Stationen zugeschriebenen Feldpoststationen der Korps, Divisionen, Brigaden, Flugverbindungen und Versorgungsstationen, um die Post an diese in verpackter Form weiterzusenden.

7 Ivan T. Peresypkin ...A v boyu eshche vazhnej. M.: Sovetskaya Rossiya, Moskva 1970. S. 72-74.

8 V. E. Zvyagincev: Vojna na vesah Femidy: Vojna 1941-1945 gg. v materialah sledstvennosudebnyh del. M.: TERRA-Knizhnyj klub, 2006. 
An den Poststationen wurde die Korrespondenz den Postboten (Spediteuren) der Armeekorps übergeben, welche sie direkt zu ihren Truppenteilen und ihren Abteilungen, die sich im Radius von bis zu 2 Kilometer von der Station befanden, überbrachten. In den Truppenteilen erhielten die Postboten Briefe und Pakete von Soldaten und Offizieren, die an die Heimat gerichtet waren, und lieferten diese an zurück an die Feldpoststationen (siehe Abbildung $3)$.

In den Realitäten des Krieges funktionierte dieses System jedoch nicht sehr effektiv. Viele Briefe wurden mit Verspätung geliefert, da Fahrzeuge mit Kennzeichen der Postdienste des Volkskommissariats der Kommunikation oft nicht auf Eisenbahnen und Straßen fahren konnten, die von militärischen Einheiten und Militärfahrzeugen besetzt waren.

Außerdem hatten die Chefs der Feldpoststationen nicht immer die richtige Qualifikation für die Arbeit unter den Bedingungen der Kriegszeit. Oft wurden Fehler in den Adressangaben gemacht, die Post wurde langsam verarbeitet, was $\mathrm{zu}$ einer Ansammlung von Briefen und Postpaketen auf Militärpostbasen und Sortierstellen führte.

Auf diese Mängel der Arbeit der Militärfeldpost wurde im Befehl Zur Arbeit der Feldpost vom 18. September 1941 reagiert. Die damit verbundene Ordnung ist als Antwort auf zahlreiche Beschwerden von Soldaten, Offizieren und politischen Funktionären der Feldarmee anzusehen. Der Befehl war auch das Ergebnis der im August durchgeführten Überprüfung der Arbeit der Verwaltungen und Abteilungen der Feldkommunikation der Fronten und Armeen sowie der Feldpoststationen. Die Arbeit der Feldpostbehörden wurde darin als »außergewöhnlich schlecht« beschrieben:

»Die Post und die Periodika werden in der Regel mit großer Verzögerung und Unterbrechungen geliefert. Auf Militärpostsortierstellen, Basen und Feldpoststationen sammelt sich eine große Anzahl von nicht zugestellter Korrespondenz. Die Verarbeitung und Weiterleitung von Geldtransfers und Paketen ist überhaupt nicht eingerichtet. Die Feldpostbehörden arbeiten in den Truppenteilen überhaupt nicht an der korrekten Schreibweise von Adressen in Briefen. Es gibt Fälle von Diebstahl von rotarmistischen Paketen und Geldtransfers «?

Dieser Befehl wurde von Peresypkin unterzeichnet. Im Text des Dokuments wurde besonderes Augenmerk auf die Bestrafung von ganz bestimmten Beamten gelegt. ${ }^{10}$ Einige Chefs wurden auf sein Geheiß degradiert.

9 Prikaz o rabote polevoj pochty // Russkij arhiv: Velikaya Otechestvennaya: T. 13 (2-2). Prikazy narodnogo komissara oborony SSSR. 22 iyunya 1941 g.-1942 g. M.: TERRA, 1997. S. 86-88.

10 Zum Beispiel, »dem Chef der 16. Abteilung der Feldkommunikation Marchenko für die Nachlässigkeit zur Ausführung von offiziellen Aufgaben, um strengen Verweis mit der Verhinderung 
Der stellvertretende Volkskommissar der Verteidigung forderte, alle Mängel in kürzester Zeit zu beseitigen und innerhalb von zehn Tagen eine rechtzeitige und reibungslose Zustellung von Briefen, Paketen und Geldtransfers zu gewährleisten.

Die Analyse des Problems der Funktionsweise der Feldpost in der Anfangsphase des Krieges sollte die besonderen Umstände berücksichtigen, unter denen sich das riesige Land und seine Streitkräfte während der Niederlagen von Sommer und Herbst 1941 und dem Rückzug der Armee in den Osten befanden. Neben den materiellen Verlusten haben sich diese Ereignisse auf die Moral der Postbeamten ausgewirkt, und dies hat zwangsläufig auch zu einem Rückgang der Qualität Ihrer Arbeit geführt. Die drastische Zunahme der Korrespondenz, die von und zu der Armee ging, war für das Personal der Feldpoststrukturen beispiellos. ${ }^{11}$

\section{Entwicklung der sowjetischen Militär-Feldpost während des Krieges}

Das andere Problem war mit der Reihenfolge der Nummerierung von Feldpoststationen in der Feldarmee verbunden. In Friedenszeiten war der Orientierungspunkt für den Postboten der Name der Straße sowie die Nummer des Hauses und der Wohnung. Mit dem Beginn des Krieges sind andere Adressangaben üblicher geworden. Auf den Postsendungen musste die Nummer der Feldpoststation, der gültige Name des Truppenteils und der Abteilung, sowie der Familienname sowie der Name und der Vatersname des Empfängers angegeben werden. Der Absender gab zudem seine detaillierte Adresse an.

Nach und nach entwickelten sich die folgenden Varianten der Adressschreibweise heraus:

Option 1: Д.К.А., 105 ппс, 158 артполк

Option 2: Д.К.А., 105 ппс, 158 ап

Option 3: Д.К.А., 105 ппс, п/я $14^{12}$.

১D.К.А.`(Д.К.А.) stand dabei für die >Aktive Rote Armee`(die Feldarmee), $>$ PPS $<$ (ппс) für die >Feld-Poststation $\prec$ und $>\Pi / я<$ für das >Postfach .

zu erklären«. In einem anderen Fall wurde als Grund für die Strafe »7 Tage Verspätung von Briefen für 20 Truppenteile« angegeben.

11 Etwa 70 Millionen Briefe und 30 Millionen Zeitungen wurden monatlich verschickt.

12 Polozhenie o sluzhbe polevoj i voenno-morskoj pochty. Central'nyj arhiv Ministerstva oborony Rossijskoj Federacii (CAMO RF). F. 219. Op. 679. D. 5. L. 12 URL: https://pamyat-naroda.ru (letzter Zugriff: 4.10.2019). 
Der Kommandeur der Verbindung (oder Vereinigung) wies dem Postfach eine Postfachnummer zu. Die Zuordnung von Feld-Poststationen-Nummern von Verbindungen und Vereinigungen wurde durch die Anordnung der Dislokations-Postabteilung des Hauptamtes der Kommunikation der der $>$ Roten Armee (RKKA) durchgeführt.

Diese Reihenfolge der Nummerierung war nachvollziehbar, aber im Falle der Eroberung der Feld-Poststation durch den Gegner konnte der Gegner nicht nur leicht die tatsächliche Nummerierung der militärischen Teile, sondern auch Ihre Dislokation herausfinden. Dies geschah an der Front ziemlich oft.

Um dieses Problem zu lösen, wurde am 5. September 1942 durch den Volkskommissar der Verteidigung der UdSSR der Befehl Nr. 0679 Zur Einführung der Anweisungen zur Adressierung der Postkorrespondenz in der Roten Armee in der Kriegszeit gegeben. Diese Anweisung implizierte ein neues Format zum Schreiben einer Adresse. Beispiel: »DKA, PPS 173, Teil 98«. Die Nummer 98 gehörte dem 32. Schützenregiment der 19. Schützendivision. Um also zu sehen, welcher militärischen Einheit diese Nummer gehörte, musste einem der Text des Befehls zur Zuweisung der Zahlen bekannt sein.

Wenn sich ein Truppenteil im Hinterland befand, musste man an den Anfang der Adresse den Namen der Stadt oder des Dorfes schreiben. Zum Beispiel »Stadt Ryazan, Teil $729 «^{13}$.

Es war zudem verboten, in der Adresse den Standort des Truppenteils oder der Feldpoststation (Agentur), den Namen der Front, die Nummer der Armee, den Namen des Armeekorps (auch nicht abgekürzt) oder die gültige Nummer des Armeekorps anzugeben. Es wurde auch verboten, die Position oder Spezialisierung und den militärischen Rang des Adressaten vor dem Schreiben des Familiennamens in der Adresse anzugeben.

Trotz dieser Umgestaltungen befanden sich die Listen der realen Nummern der Truppenteile bei vielen Mitarbeitern der Organe der Feldpost des Volkskommissariats der Kommunikation. Dieser Umstand erhöhte die Gefahr der Identifizierung der Dislokation von Truppenteilen durch den Gegner.

Gemäß des Befehls des Volkskommissars der Verteidigung der UdSSR vom 6. Dezember 1942 Zur Reorganisation von Organen des Postdienstes der Roten Armee und der Feldpost wurden die Organe der Militärfeldpost von der Struktur des Volkskommissariats der Kommunikation an das Hauptamt der Kommunikation der Roten Armee übertragen. Das Zentrale Militäramt für Feldkommunikation des Volkskommissariats der Kommunikation wurde in das Amt

13 Prikaz Narodnogo Komissara Oborony Soyuza SSR № 0679. URL: http://www.soldat.ru/ doc/nko/text/1942-0679.html (letzter Zugriff: 6.10.2019). 
der Militärfeldpost des Hauptamtes der Kommunikation der Roten Armee umgewandelt.

Die Nummerierung der Truppenteile erfolgte nun in der Abteilung der Dislokation der Truppen der Roten Armee als Teil des Organisationsamtes des Generalstabs und der ihm untergebenen Instanzen ${ }^{14}$.

Daten über die Dislokation der Truppen von Fronten, Bezirken und Armeen wurden zu streng geheimen Dokumenten erklärt. Für die Speicherung dieser Daten im Geheimen waren die Hauptquartiere verschiedener Ebenen verantwortlich.

Die Regierung und das Kommando der Roten Armee sahen jedoch selbst diese strengen Maßnahmen zur Sicherung der Geheimhaltung als unzureichend an. Am 6. Februar 1943 gab es einen neuen Befehl zur Organisation der Arbeit der Feldpost.

Dieser Befehl führt erneut eine neue Ordnung der Nummerierung der Feldpoststationen von Truppenteilen und Militärbehörden ein. Allen Truppenteilen und Verbindungen der Roten Armee wurden fünfstellige Nummern zugewiesen.

Die neuen Adressen der Truppenteile sahen nun zum Beispiel so aus: »Truppenteil Feld Post 10952«. Diese Nummer gehörte der 15. beweglichen Reparaturbasis der 50. Armee ${ }^{15}$.

Diese Reihenfolge der Nummerierung der Truppenteile, um den Adressaten eines Briefes oder eines Postpakets anzuzeigen, entstand unter dem Einfluss der Erfahrungen der Wehrmacht, die von sowjetischen Spezialisten sorgfältig untersucht wurden. Die neuen Regeln erwiesen sich schnell als wirksam und wurden bis zum Ende des Zweiten Weltkrieges nicht mehr geändert.

Im Januar 1943 wurden jedoch Maßnahmen ergriffen, um den Postverkehr zu beschleunigen. Der Befehl des Volkskommissars der Verteidigung der UdSSR Nr. 075 vom 21. Januar regelte Transport und Lieferung der militärischen Postkorrespondenz ${ }^{16}$. Laut diesem war es beispielsweise streng verboten, mit Fahrzeugen, auf denen militärische Postkorrespondenz geliefert wurde, auch andere Dinge zu transportieren (siehe Abbildung 5). Dadurch wurde der Posttransport explizit priorisiert und die rechtzeitige Lieferung von Postgütern gewährleistet. Es sollte angemerkt werden, dass die Mengen der Posttransporte während des Krieges kolossal waren: etwa 70 Millionen Briefe wurden monat-

$14 \mathrm{O}$ reorganizacii organov dislokacionno-pochtovoj sluzhby Krasnoj Armii i voenno-polevoj pochty // Russkij arhiv: Velikaya Otechestvennaya: T. $13(2-2)$. Prikazy narodnogo komissara oborony SSSR. 22 iyunya 1941 g. - 1942 g. M.: TERRA, 1997. S. 374-377.

15 Spravochnik polevyh pochtovyh stancij RKKA v 1941-1945 godah. URL: http://soldat.ru/ pps_about.html.

16 Prikaz Narodnogo Komissara Oborony Soyuza SSR № 075. URL: http://www.soldat.ru/doc/ nko/text/1943-075.html (letzter Zugriff: 6.10.2019). 
lich gesendet. Die Anzahl der Briefe, die von der Armee in das Hinterland geschickt wurden, betrug 2 Milliarden 794 Millionen. Das bedeutet durchschnittlich 82 Briefe für jeden der 84 Millionen sowjetischen Soldaten und Offiziere (siehe Abbildung 4). ${ }^{17}$

Der Weg eines einzelnen Briefes, der von einem Soldaten der Feldarmee geschrieben wurde, kann so beschrieben werden: Der Brief wurde an den militärischen Postboten oder an die Militärpoststation der Militärverbindung übertragen. Dann ging die Postkorrespondenz zur Militärpostbasis der Armee, um anschließend zur Militärpostsortierstelle der Front übermittelt zu werden. Danach wurden die Briefe an den Austauschpunkt gesendet, wo sie auf Postwagen, Flugzeuge oder Dampfer des Volkskommissariats der Kommunikation verladen wurden. Erst von da aus gelangte der Brief auf dem üblichen Weg zu den Büros der zivilen Post.

Ein weiteres Problem war die Umgestaltung innerhalb der Sowjetunion von der Friedens- zur Kriegswirtschaft. Die Militarisierung der Industrie hatte dazu geführt, dass die Produktion vieler Arten von zivilen Produkten reduziert oder vollständig gestoppt wurde. Eine Folge dieser Situation war der Mangel an Briefumschlägen. Als Ergebnis erschien das sogenannte >Dreieck besonderer Weise gefalteter Brief, der sicherlich eines der Symbole des Großen Vaterländischen Krieges wurde (siehe Abbildung 6).

Auf der anderen Seite erleichterte der Mangel an Umschlägen die Überprüfung der Inhalte von Briefen durch die Behörden der militärischen Zensur. In den Briefen war es verboten, irgendwelche Daten zu melden, die die Dislokation oder die Nummer des Truppenteils offenbaren könnten. Es war zudem auch untersagt, negative Informationen über die aktuelle Situation an der Front und in der Heimat zu verbreiten ${ }^{18}$.

Die technischen Besonderheiten des Funktionierens der Feldpost haben das Erscheinen der neuen kulturellen Phänomene in der sowjetischen Gesellschaft in besonderem Maße geprägt. So wurde der Dreiecksbrief zu einem der Symbole des Zweiten Weltkrieges, das sich sowohl im gesellschaftlichen Bewusstsein als auch der schöngeistigen Literatur widergespiegelt, ${ }^{19}$ da für die Menschen, die den Erhalt solcher Briefe erwarteten, ihre Zustellung eine enorme Bedeu-

17 A. E. Larionov: Frontovaya povsednevnost' Velikoj Otechestvennoj vojny: social'nye kommunikacii i duhovnaya zhizn' RKKA 1941-1945 gg. M.: Chastnoe izdatel'stvo "Zolotoe sechenie«, 2015. S. 203.

18 Fomenko M. Vam pis'mo! URL: https://warspot.ru/12562-vam-pismo (letzter Zugriff: 05.10. 2019).

19 Asadov E. Zarnicy vojny. Povest'. Stihi. Poemy. Moskva: Voennoe izd-vo, 1989; Mityaev A.V. Pis'mo s fronta. Rasskazy o Velikoj Otechestvennoj Vojne. M.: Belyj gorod, 2017; Simonov K.M. Voennaya lirika. Gor'kij: Volgo-Vyatskoe knizhnoe izdatel'stvo, 1972. 
tung hatte. Man kann behaupten, dass die Arbeit der Feldpost die Mentalität und die Werte der sowjetischen Menschen unmittelbar beeinflusste.

In der Schlussphase des Zweiten Weltkriegs war die Rote Armee tief in das Territorium der europäischen Länder vorgestoßen. Unter der Kriegsbeute, die von sowjetischen Soldaten und Offizieren mitgenommen wurde, gab es oft eine Vielzahl von Postkarten. Sie enthielten Bilder (u.a. Stadtansichten und Bilder von Frauen), die sich von den in der Sowjetunion bekannten Produkten unterschieden $^{20}$. Die Regierung und das Kommando der Roten Armee verhinderten nicht die Verwendung solcher Postkarten. Somit sind viele von ihnen, die gerade gegen Ende des Krieges vermehrt verschickt worden waren, bis heute in den Familienarchiven erhalten geblieben.

\section{Zusammenfassung}

Insgesamt lässt sich sagen, dass der postalische Dienst den Forderungen der Kriegszeit angepasst war. Dies wurde durch energische und systematische Handlungen des Kommunikationskommissars von Peresypkin und seine unmittelbaren Untergebenen erleichtert. Die schrittweise Reorganisation der Machtstrukturen, die für die Arbeit der militärischen Feldpost verantwortlich waren, führte zu einer klaren Abgrenzung der Befugnisse und der Verantwortung zwischen den militärischen Feldkommissariaten und den Zivilbehörden, die dem Volkskommissariat für Kommunikation unterstellt waren. Auf der Grundlage der Analyse der Erfahrung der Wehrmacht wurde die Reihenfolge der Nummerierung von Adressen von militärischen Einheiten und Verbindungsstellen entwickelt und eingeführt, da sie am besten für das Funktionieren unter Kriegsbedingungen geeignet war. Alle diese Aktivitäten haben die Effizienz der Arbeit des Militärpostdienstes erheblich verbessert.

\section{Quellen}

Polozhenie o sluzhbe polevoj i voenno-morskoj pochty. Central'nyj arhiv Ministerstva oborony Rossijskoj Federacii (CAMO RF), F. 219. Op. 679. D. 5. L. 10. URL: https:// pamyat-naroda.ru (letzter Zugriff: 14.02.2020).

Polozhenie o sluzhbe polevoj i voenno-morskoj pochty, Central'nyj arhiv Ministerstva oborony Rossijskoj Federacii (CAMO RF), F. 219. Op. 679. D. 5. L. 12. URL https:// pamyat-naroda.ru (letzter Zugriff: 04.10.2019).

20 Fomenko M. Vam pis'mo! URL: https://warspot.ru/12562-vam-pismo (letzter Zugriff: 05.10. 2019). 


\section{Literaturverzeichnis}

Yu. P Agafonov: Soldatskij treugol'nik. Velikaya Otechestvennaya vojna v sud'bah zaural'cev, Shadrinsk 2001.

M. Fomenko: Vam pis'mo!, URL: https://warspot.ru/12562-vam-pismo (letzter Zugriff: 05.10.2019).

A. I. Gluhov: Zapiski polkovogo pochtal'ona, St. Petersburg 2005.

V. N. Kuselenkova (Hrsg.): Komandnyj i nachal'stvuyushchij sostav Krasnoj Armii v 19401941 gg.: Struktura i kadry central'nogo apparata NKO SSSR, voennyh okrugov i obshchevojskovyh armij: Dokumenty i materialy, Moskau/St. Petersburg 2005.

A. E. Larionov: Frontovaya povsednevnost' Velikoj Otechestvennoj vojny: social'nye kommunikacii i duhovnaya zhizn' RKKA 1941-1945 gg, Moskau 2015. S. 203.

A.V. Mityaev: Pis'mo s fronta. Rasskazy o Velikoj Otechestvennoj Vojne, Moskau 2017.

Ivan T. Peresypkin: ...A v boyu eshche vazhnej, Moskau, Sovetskaya Rossiya, 1970, S. 72-74.

I. T. Peresypkin: Voennaya radiosvyaz, Moskau 1962.

O reorganizacii organov dislokacionno-pochtovoj sluzhby Krasnoj Armii i voenno-polevoj pochty, Prikazy narodnogo komissara oborony SSSR. 22 iyunya 1941 g. - 1942 g, Russkij arhiv: Velikaya Otechestvennaya: T. 13 (2 - 2), Moskau 1997. S. 374-377.

Prikaz Narodnogo Komissara Oborony Soyuza SSR № 0679. URL: http://www.soldat.ru/ doc/nko/text/1942-0679.html (letzter Zugriff: 06.10.2019).

Prikaz o rabote polevoj pochty, Prikazy narodnogo komissara oborony SSSR. 22 iyunya 1941 g. - 1942 g, Russkij arhiv: Velikaya Otechestvennaya: T. 13 (2 - 2), Moskau 1997, S. 8688.

Prikaz Narodnogo Komissara Oborony Soyuza SSR № 075, URL: http://www.soldat.ru/doc/ nko/text/1943-075.html (letzter Zugriff: 06.10.2019).

Konstantin M. Simonov: Raznye dni vojny. Dnevnik pisatelya, Moskau 1982, URL: http://militera.lib.ru/db/simonov_km/index.html (letzter Zugriff: 04.10.2019).

Konstantin Simonov: Kriegstagebücher, DDR-Lizenzausgabe, München 1979.

K.M. Simonov: Voennaya lirika. Gorki 1972.

Spravochnik polevyh pochtovyh stancij RKKA v 1941-1945 godah, URL: http://soldat.ru/ pps_about.html.

o. V.: Iskusstvo pobezhdat: Polkovodcy Vtoroj Mirovoj vojny, Moskau 2005.

o. V.: Polkovodcy i voenachal'niki Velikoj Otechestvennoj, Moskau 1985.

o. V.: Vojska svyazi Sovetskoj Armii: Kratkij ocherk, Moskau 1948.

Günter Warm: Konstantin Simonow, in: Urania Universum, Jg. 19, 1973, S. 415-422.

Asadov E. Zarnicy: vojny. Povest'. Stihi. Poemy, Moskau 1989.

Yu. A. Zhukov: Soldatskie dumy: o chyom rasskazyvali pis'ma s frontov Velikoj Otchestvennoj vojny, Moskau, Sovetskaja Rossiya, 1987.

V. E. Zvyagincev: Vojna na vesah Femidy: Vojna 1941 - 1945 gg. v materialah sledstvennosudebnyh del, Moskau 2006. 
Open-Access-Publikation im Sinne der CC-Lizenz BY-NC-ND 4.0 (C) 2021, Vandenhoeck \& Ruprecht GmbH \& Co. KG, Göttingen ISBN Print: 9783847112280 - ISBN E-Lib: 9783737012287 


\section{Abbildungen}

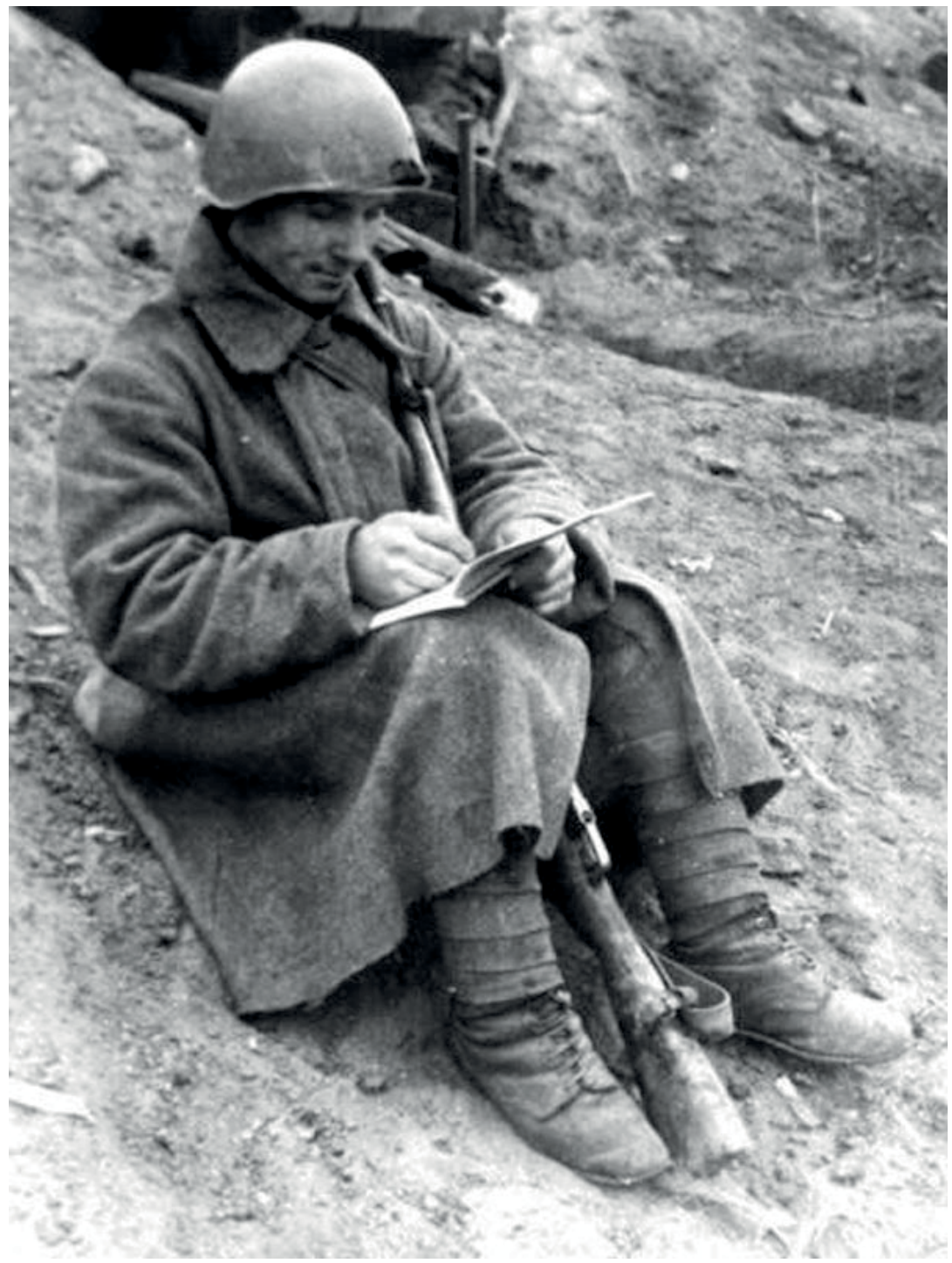

Abbildung 1: Ein sowjetischer Soldat schreibt einen Brief in der Pause zwischen den Kämpfen. 


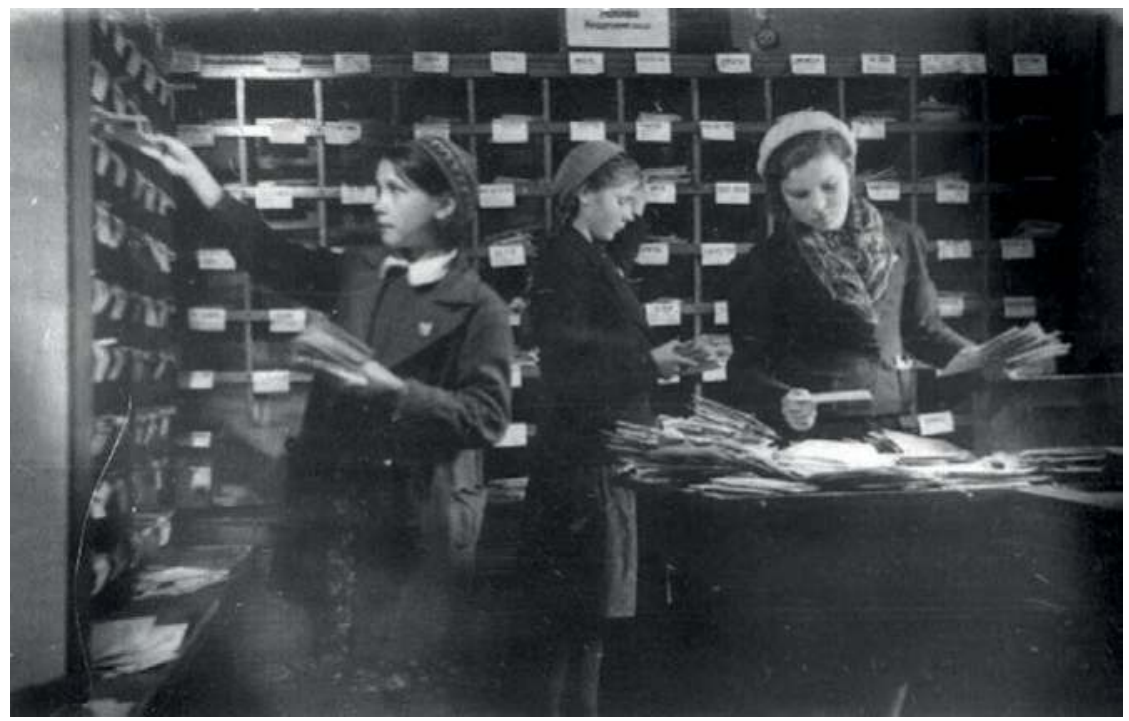

Abbildung 2: Sortieren von Briefen in der Post.

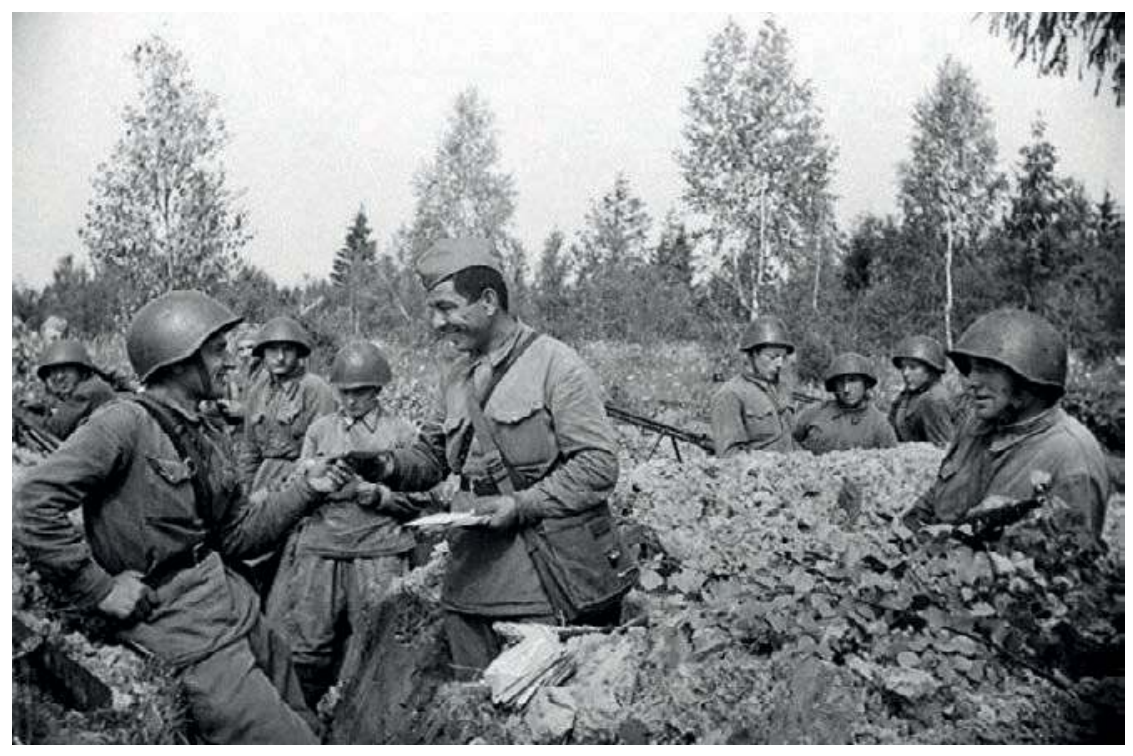

Abbildung 3: Übergabe von Briefen an sowjetische Soldaten in der Nähe der Frontlinie. 


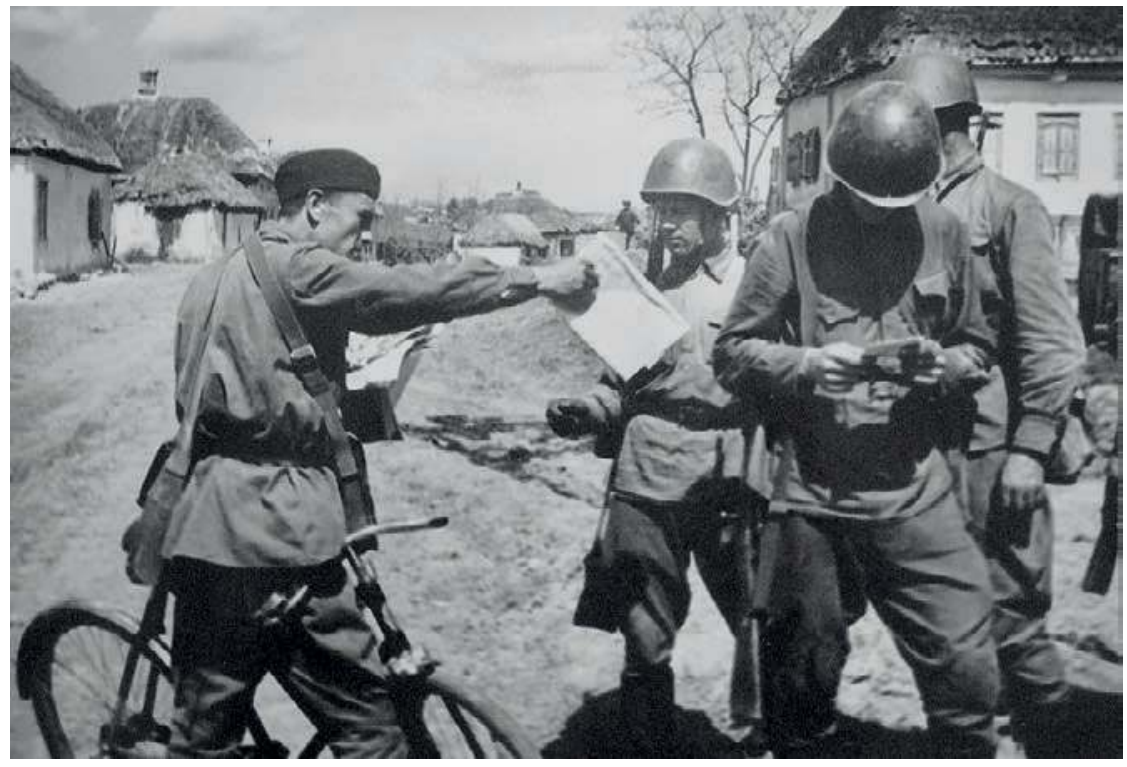

Abbildung 4: Feldpost. 1942. Der Urheber des Bildes ist der Fotograf Anatoly Garanin.

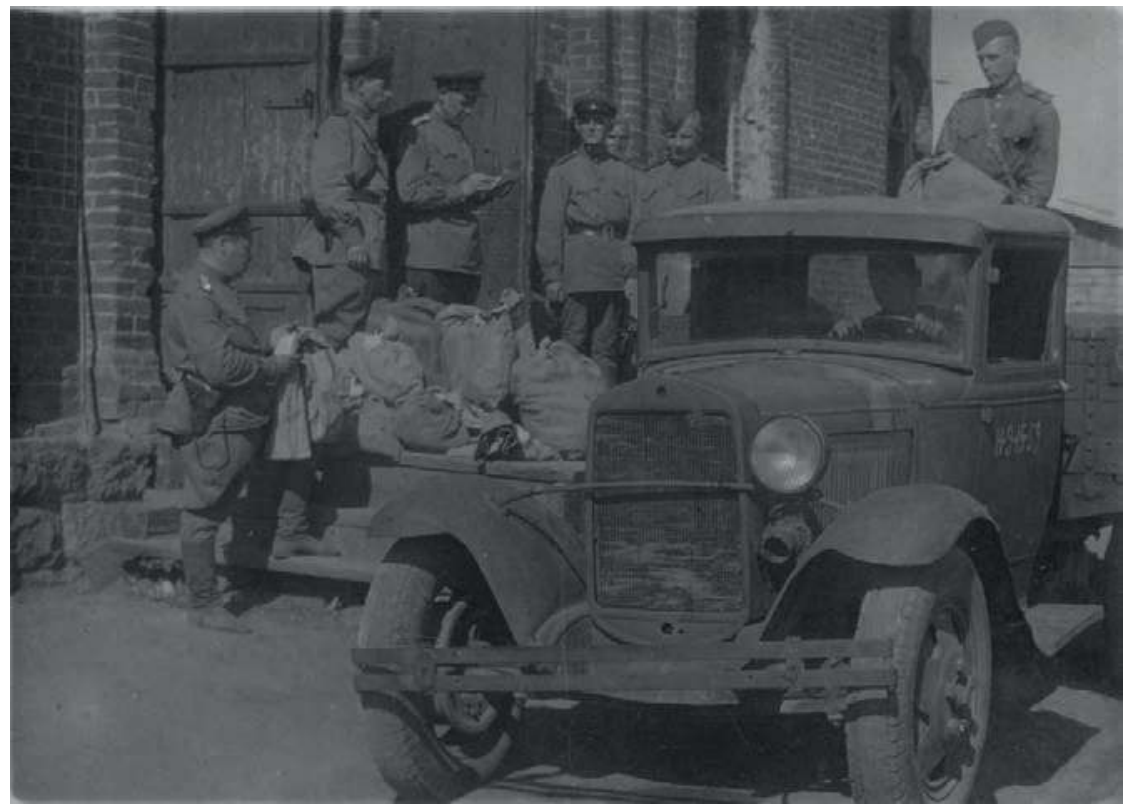

Abbildung 5: GAZ-AA - LKW des Militärpostdienstes. 


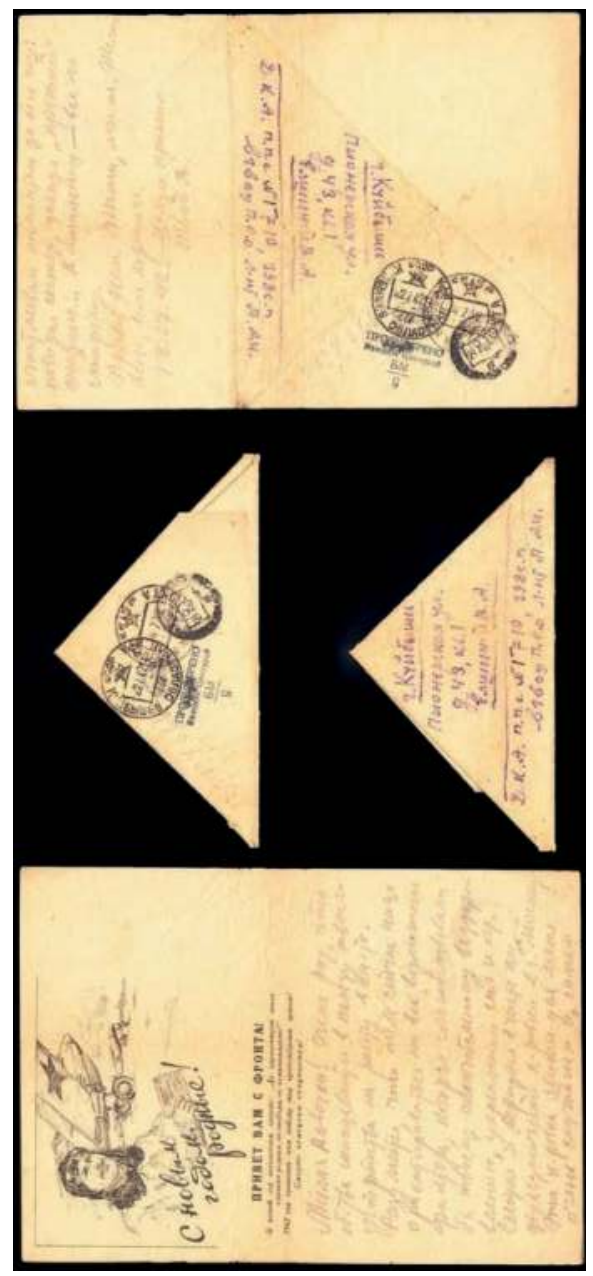

Abbildung 6: Briefbogen in typischer dreieckiger Faltung 
Die Post der Häftlinge in Konzentrationslagern ${ }^{1}$

\section{Die einzige Verbindung zur Außenwelt}

Für die Häftlinge der Konzentrationslager war Post, von wenigen Besuchen in Einzelfällen abgesehen, die einzige Verbindung zur Außenwelt.

Erlaubt war dem Häftling die Korrespondenz nur mit einem bezeichneten und registrierten Angehörigen. Dieses war in der Regel der Ehepartner oder ein Elternteil. In der Kommandantur führte die Zensurstelle über den Postverkehr jedes Häftlings mittels einer >Postkontrollkarte` genau Buch.

Für ihre Korrespondenz mussten die Häftlinge in der Regel die offiziellen Vordrucke des jeweiligen Lagers verwenden. ${ }^{2}$ Wenige privilegierte Häftlinge durften neutrales Briefpapier verwenden, so die >Protektoratshäftlinge $<$, Mitglieder der tschechischen politischen und kulturellen Elite, die als Geiseln für den Fall von Widerstand im Protektorat ins Konzentrationslager Buchenwald deportiert worden waren.

Als Korrespondenzsprache war für die Häftlinge ausschließlich Deutsch zugelassen. Für die zahlreichen Menschen aus den überfallenen Ländern, die die deutsche Sprache nicht beherrschten, war dies eine zusätzliche Härte. Meist fanden sich deutsche oder Deutsch sprechende Kameraden, die ihnen halfen. Häufig verlangten diese Kameraden eine Gegenleistung für die Übersetzungshilfe. Und die bestand im Kostbarsten, das ein Häftling besitzen konnte: Brot.

1 Der nachfolgende Beitrag ist die überarbeitete Fassung des Kapitels »Post der Häftlinge« aus Heinz Wewer: Spuren des Terrors. Postalische Zeugnisse zum System der deutschen Konzentrationslager, Berlin/Leipzig 2020, S. 133-150.

2 Von den Vordrucken gibt es eine kaum zu überblickende Zahl von Postkarten, Briefumschlägen und Faltbriefen, die sich auf vielfältige Art voneinander unterscheiden und die international als Sammel- und Ausstellungsgebiet großes Interesse finden. Auf die allein für Philatelisten interessanten Typen, Unterscheidungsmerkmale und Verwendungszeiträume wird in dieser Arbeit nicht eingegangen. Hierzu verweist der Autor auf den umfassenden Katalog von Erik Lørdahl. Vgl. Erik Lørdahl: German Concentration Camps 1933-1945. History and Inmate Mail, 2 Bde., davon Bd. II in zwei Teilen, Tårnåsen (Norwegen) 2000. 
Schreiben durften Häftlinge nur an festgelegten `Schreibtagen`. In Dachau war dies abwechselnd ein Sonntag für Häftlinge der Blöcke mit geraden Zahlen und für Häftlinge der Blöcke mit ungeraden Zahlen. Überstieg die Zahl der abgehenden oder hereinkommenden Sendungen eines Häftlings die von den jeweils geltenden Bestimmungen erlaubte, ging die überzählige Sendung zurück wie im Falle des Briefes in Abbildung 1 oder wurde, zumeist ohne Benachrichtigung von Absender und Adressat, vernichtet.

Der Inhalt der hereinkommenden und auch der hinausgehenden Postkarten und Briefe wurde von der Postzensurstelle in der Kommandantur streng zensiert, die hinausgehende Post auch vom Blockführer oder dem von diesem beauftragten Blockältesten einer zensurartigen Durchsicht unterzogen. Die Durchsicht der hinausgehenden Post durch den Blockführer oder den Blockältesten stärkte deren ohnehin schon beträchtliche Macht, denn sie konnten Postsendungen, deren Inhalt für den Absender potenziell gefährlich war, zurückhalten, oder sie konnten sie an die Zensurstelle weitergeben und so den Absender ans Messer liefern. Verstöße gegen die Zensurbestimmungen zogen Beschlagnahme der betreffenden Postsendung und Bestrafungen des Häftlings nach sich. Von den Bestrafungen war die Postsperre, also das Vorenthalten der Postteilnahme, noch die mildeste. Es sind aber auch Fälle bekannt, in denen Häftlinge wegen einer unbedachten Äußerung in einem Brief - etwa »auch für uns scheint die Sonne wieder « - zu 25 Stockhieben verurteilt wurden. ${ }^{3}$

In einer Dienstanweisung des Kommandoführers des Konzentrationslagers Buchenwald mit der Bezeichnung »Verhalten beim Briefe-Schreiben« heißt es:

»5. Angaben über den Aufenthaltsort sowie über die Arbeitsverhältnisse sind verboten! $[\ldots]$

8. Äußerungen über Fliegerangriff, Krankheit, Politik usw. sind verboten. Auch Angaben über das Lagerleben.

9. Wird eine obengenannte Anordnung nicht befolgt, so hat dies die Vernichtung des Briefes zur Folge. Ersatzbriefe gibt es nicht. Außerdem erfolgt eine Bestrafung. ${ }^{4}$

Ob diese Anweisung den Häftlingen bekannt gemacht wurde oder ob sie eine interne Richtschnur der SS war, ist nicht überliefert.

Die Gefangenen lernten in der Regel schnell, welche Themen tabu und in der Korrespondenz zu vermeiden waren. Nicht selten halfen alteingesessene Gefangene ihren neu eingelieferten Kameraden, indem sie Brieftexte vor Absendung inhaltlich redigierten. Von der Zensur beanstandete Texte wurden im

3 Aus den Erinnerungen des früheren Auschwitz-Häftlings Ryszard Kordek, zitiert in: Tadeusz Iwasko: Kontakt mit der Außenwelt, in: Wacław Długoborski, Francisek Piper (Hrsg.): Auschwitz 1940-1945, Bd. 2, Oświęcim 1999, S. 513.

4 Copy of Doc. No. 82084956\#1 (1.1.5.0/0065/0021) in conformity with the ITS Archives. 
besten Fall unlesbar gemacht (Abbildung 2), im schlechteren Fall wurden sie entweder vernichtet oder als Fragmente - die beanstandeten Stellen waren mit der Schere herausgeschnitten (Abbildung 3) - den Adressaten zugestellt. Das Aufbewahren größerer Mengen von Post war den Gefangenen verboten. Nur die zuletzt eingetroffene Postsendung durften sie aufbewahren, und das auch nur für begrenzte Zeit. Neben den Lebensbedingungen der Häftlinge, die die Aufbewahrung von Papier nicht begünstigten, dürfte diese Vorschrift der Grund sein, weshalb wenig Post an Häftlinge erhalten geblieben ist, weit weniger als Post von Häftlingen.

Aufgrund der Zensur und der Selbstzensur ist der Informationsgehalt der Mitteilungen sehr dürftig. Man erfährt aus dieser Post nichts über die Verhältnisse im Lager, über das wirkliche Befinden der Gefangenen und umgekehrt nichts über Probleme in der Außenwelt oder den Gang des Krieges. Die Mitteilungen liegen stets auf der persönlichen Ebene. Sie sind aber oft von einer subtilen Emotionalität, etwa in der Art, wie Ehepartner einander ansprechen, wie sie ihre Sehnsüchte und ihre Liebe mitteilen, wie sie sich über gemeinsame Kinder austauschen. Die postalische Kommunikation war für die Beteiligten ohne Zweifel von hohem Wert. Man kann das daran erkennen, welche Freude der Empfang einer Postsendung und welche Beunruhigung deren Ausbleiben auslöste. Hierzu Walter Poller in seinem bewegenden Bericht über die Zeit als Gefangener im Konzentrationslager Buchenwald:

"Wir waren im Lager so grenzenlos einsam, und selbst da, wo sich engere Freundschaftsbande knüpften, blieb noch ein kleiner Rest Fremdheit übrig. Unsere Frauen, unsere Kinder, unsere Freunde daheim, alle jene Menschen, mit denen wir uns unlösbar verbunden fühlten, sie waren im innersten Herzen unser kostbarster Besitz. Die immer neu aufkommende Hoffnung, daß uns das Schicksal es doch eines Tages gönnen könnte, wieder mit diesen Menschen zusammen zu sein, riß uns immer und immer wieder empor. [...] Und darum war jede Zeile von daheim, die Ausdruck dieser Bindung war, für uns Todumwehte und Todgeweihte ein seelisches Erlebnis von solcher Köstlichkeit und Eindringlichkeit, von solcher inneren Stärkung, daß die begnadetsten Worte ihre tiefen Werte nicht darzustellen vermögen. $\aleph^{5}$

Der polnische Historiker und ehemalige Auschwitz-Häftling Tadeusz Szymański schreibt in seinem Aufsatz über Briefe aus Konzentrationslagern:

»Wer die nationalsozialistische Okkupation Polens nicht aus eigener leidvoller Erfahrung kennt, der vermag sich heute nur schwer vorzustellen, welche Bedeutung ein Brief aus dem Gefängnis oder aus dem KZ für die Familie hatte, wie wichtig es für die Häftlinge im $\mathrm{KZ}$ war, wenn sie einen Brief von ihren Familienangehörigen ausgehändigt bekamen, und

5 Walter Poller: Arztschreiber in Buchenwald, Offenbach a. M. 1960 (zuerst 1946), S. 274 f. 
welche Bedeutung solche Nachrichten für die hinter Gefängnismauern und Stacheldraht eingesperrten Menschen hatten. ${ }^{6}$

Hans Maršálek, ein Überlebender des Konzentrationslagers Mauthausen, beschreibt in seinem Standardwerk zur Geschichte Mauthausens die Regelungen, nach denen unterschiedliche Gruppen am Postverkehr teilnehmen oder nicht teilnehmen durften. Er schließt: »Die Briefe von Zuhause waren Bausteine der Hoffnung und unersetzlicher Ansporn nicht aufzugeben. « ${ }^{7}$

Oder Arnold Weiss-Rüthel in dem Bericht über seine Haft im Konzentrationslager Sachsenhausen:

»Für den Häftling, der jahrelang keine Möglichkeit hatte, seine Angehörigen wiederzusehen und auch niemals wußte, ob er sie überhaupt jemals wiedersehen würde, bedeutete ein Brief unendlich viel. Das Warten auf die Post war eine erregende Vorfreude - der Brief selbst ein hohes und tröstliches Glück. Man wurde nervös, wenn der erwartete Brief nicht kam, eine ganze Woche konnte einem dadurch zur Qual werden. Denn wir hatten nicht viel, woran wir uns freuen konnten. $\aleph^{8}$

Über die Wirkung, die das Ausbleiben von Post auf die Gefangenen ausübte, schreibt Eugen Kogon, Überlebender von Buchenwald: »Dieser schreckliche Nervenkrieg stellte eine der zermürbendsten Belastungen des Lagers dar. « ${ }^{9}$

Die Post von geliebten Menschen war für viele Häftlinge das letzte Refugium in der Tristesse und Bedrohlichkeit des Lageralltages. Sie konnte zumindest einen Hauch von Privatheit und menschlicher Wärme vermitteln. Blieb ein erwarteter Brief aus, so konnte das leicht Enttäuschungen und panikartige Besorgnisse verursachen, die über das im »normalen Leben« übliche Maß hinausgingen. So schrieb Stanislav Schön aus dem Konzentrationslager Hamburg-Neuengamme am 7. Januar 1945 an Anna Schönová in Olmütz, Text vom Autor sprachlich leicht korrigiert:

"...Ich danke Dir für Pakete, welche ich in Ordnung erhalten habe, aber es tut mir leid, dass so viele einzelne Briefe von Dir nicht kommen. Ich weiß nicht, was mit Dir los ist. Bist Du krank, oder hast Du keine Zeit, einen Brief für mich zu schreiben? Ich weiß nicht, was ich mir denken soll... ${ }^{10}$

6 Tadeusz Szymański: Briefe an die nächsten Angehörigen - Lebenszeichen aus Auschwitz, in: Jadwiga Mateja (Red.): Znaki Pamięci. Korespondencja obozowa więźniów KL Auschwitz Briefmarken Zeichen des Gedenkens. Häftlingsbriefe aus dem KL Auschwitz, Oświęcim 1995, S. 19.

7 Hans Maršálek: Die Geschichte des Konzentrationslagers Mauthausen, Wien 2006 (zuerst 1974), S. 70.

8 Arnold Weiss-Rüthel: Nacht und Nebel. Ein Sachsenhausen-Buch, Berlin/Potsdam 1949, S. 95.

9 Eugen Kogon: Der SS-Staat, Frankfurt a. M. o. J. (zuerst 1946), S. 130.

10 Original in der Sammlung des Autors. 
Bei aller Begrenztheit der Kommunikationsinhalte war es für die Lebenswirklichkeit der Häftlinge, vor allem ihre innere Resistenzfähigkeit, von großer Bedeutung, ob sie Post empfangen und absenden durften. Daher ist die Frage, in welchem Umfang und unter welchen Bedingungen Gefangene am Postverkehr teilnehmen konnten und welche Häftlinge nur eingeschränkt oder gar nicht daran teilnehmen durften, nicht nur für die philatelistische, sondern auch für die historische Forschung von Interesse.

\section{Von der deutschen Geschichtswissenschaft vernachlässigt}

Es verwundert, wie wenig Aufmerksamkeit und Genauigkeit die deutsche Geschichtswissenschaft, von Eugen Kogon abgesehen, der für die Häftlinge wichtigen Frage der Außenkontakte zugemessen hat. So heißt es in dem frühen Standardwerk System der NS-Konzentrationslager 1933-1939 von Klaus Drobisch und Günter Wieland:

»Der Kontakt der Häftlinge zu ihren Angehörigen war sporadisch. In Sachsenburg durften sie anfänglich jede Woche, in Dachau wöchentlich abwechselnd eine Karte und einen Brief, in der Lichtenburg, in Moringen, Oranienburg und Sonnenburg alle zwei, in Brandenburg und Esterwegen nur alle vier Wochen schreiben und Post empfangen, allerdings nur, wenn nicht als $>$ Strafe` Postsperre verhängt wurde. $^{11}$

Die Unterschiedlichkeit der Regeln von Lager zu Lager, von Häftlingsgruppe zu Häftlingsgruppe und über die Zeit wird hier sehr pauschal und wenig informativ abgehandelt, und die Behauptung, die Gefangenen hätten »in Dachau wöchentlich abwechselnd eine Karte und einen Brief « schreiben dürfen, ist weder von den überlieferten Bestimmungen noch vom dokumentarischen Material gedeckt. ${ }^{12}$ Nach den auf den Vordruck-Karten und -Briefen gedruckten Bestimmungen durften sie entweder einen Brief oder eine Karte erhalten und absenden.

Obwohl die Geschichte Dachaus in den Jahren 1933 und 1934 das Thema seines Dachau-Buches ist, faksimiliert Richardi ein vorgedrucktes Briefformular aus dem Jahr 1942. ${ }^{13}$ Das Beispiel ist unzutreffend. Denn im Jahr 1942 gal-

11 Klaus Drobisch, Günther Wieland: System der NS-Konzentrationslager 1933-1939, Berlin 1993, S. 115.

12 Auch in Buchenwald soll die Regel bestanden haben, dass Häftlinge abwechselnd einen Brief und eine Karte schreiben durften. So heißt es im Buchenwald-Report: »An seine unmittelbaren Angehörigen konnte der Häftling abwechselnd alle 14 Tage einen Brief und eine Karte mit vorgeschriebener Zeilenzahl schreiben.« David A. Hackert (Hrsg.): Der Buchenwald-Report, München 1996 (zuerst The Buchenwald Report, Boulder/San Francisco/Oxford 1995), S. 81.

13 Hans-Günter Richardi: Schule der Gewalt. Die Anfänge des Konzentrationslagers Dachau, München 1982, S. 294. 
ten in Dachau andere Regeln zum Postverkehr der Gefangenen als in Richardis Berichtszeitraum 1933/34. Richardi ist entgangen, dass sich in Dachau die postalischen Bedingungen, denen die Gefangenen unterlagen, im Zeitverlauf veränderten. Er stellt auch nicht die Frage, auf welche Lagerordnung sich der auf den Postformularen gedruckte »Auszug aus der Lagerordnung « eigentlich bezogen hat.

Ein weiteres Beispiel für den oberflächlichen Umgang mancher deutscher Historikerinnen und Historiker mit den Bestimmungen zum Postverkehr, denen die Gefangenen unterlagen, ist die im Übrigen verdienstvolle Arbeit von Elke Suhr, Die Emslandlager. Sie schreibt, „Briefe und Postkarten durften laut Lagerordnung des KZ Esterwegen nur zweimal im Monat geschrieben bzw. empfangen werden, nach Kriegsbeginn sogar [...] nur noch einmal innerhalb von sechs Wochen. ${ }^{14}$ Als Abbildung bringt die Autorin hierzu eine Vordruckpostkarte von Carl von Ossietzky aus dem Konzentrationslager Esterwegen, Poststempel Esterwegen, 21. Dezember 1935, an seine Frau Maud. ${ }^{15}$ In dem vorgedruckten Text der Postkarte heißt es unter der Zeile »Auszug aus der Lagerordnung«: »Jeder Häftling darf in einer Woche einen Brief oder eine Postkarte empfangen und auch absenden. [...] «, also durfte er pro Monat je vier Sendungen abschicken und empfangen. Der Widerspruch zwischen ihrem Text und dem abgebildeten Dokument ist der Autorin verborgen geblieben, ganz abgesehen von der unzutreffenden Behauptung, "nach Kriegsbeginn" sei nach der Lagerordnung des KZ Esterwegen nur noch einmal innerhalb von sechs Wochen der Empfang und die Absendung von Post erlaubt gewesen. Bekanntlich hat das Konzentrationslager Esterwegen "nach Kriegsbeginn " nicht mehr bestanden, denn Himmler hat es im Sommer 1936 aus strategischen Gründen aufgelöst und die Insassen nach Sachsenhausen verlegen lassen. Die Baulichkeiten wurden danach als Strafgefangenenlager genutzt.

Auch in neueren Publikationen deutscher Historiker, in denen über den Alltag der KZ-Gefangenen berichtet wird, taucht das Thema Teilnahme am Postverkehr zumeist nur beiläufig oder gar nicht auf. ${ }^{16}$ Vielleicht erscheinen

14 Elke Suhr: Die Emslandlager. Die politische und wirtschaftliche Bedeutung der emsländischen Konzentrations- und Straflager 1933-1945, Bremen 1985, S. 96 f.

15 Auf der Abbildung ist das Datum des Poststempels nicht zu erkennen. Die Datierung dieser Karte ergibt sich aus der Reproduktion derselben Karte auf dem Umschlagbild von Heinrich Heeren: Der Postverkehr mit den Emslandlagern 1870 bis 1950, Papenburg 2006.

16 So etwa in Günter Morsch, Susanne zur Nieden (Hrsg.): Jüdische Häftlinge im Konzentrationslager Sachsenhausen 1936-1945, Berlin 2004 oder Stanislav Zámečnik: Das war Dachau, Frankfurt a. M. 2010 (zuerst 2002). Auch in dem neunbändigen Standardwerk von Wolfgang Benz, Barbara Distel (Hrsg.): Der Ort des Terrors, wird zumeist nur beiläufig auf die Teilnahme der Gefangenen am Postverkehr eingegangen. Eine Ausnahme bildet der Beitrag von Barbara Distel über die Lebensbedingungen im Stammlager Auschwitz. Die Autorin fasst hier in einem Absatz die wichtigsten Informationen zum Thema zusammen. Barbara Distel: Lebensbedingungen im 
den Autoren Karten und Briefe, die die Gefangenen schreiben und erhalten oder nicht schreiben und erhalten und Pakete, die sie erhalten oder nicht erhalten durften, angesichts der täglichen Lebensbedrohung, der sie ausgesetzt waren, als trivial oder quantité négligeable. Viele Überlebende haben aber, wie gezeigt, bekundet, dass der Empfang von Post sehr wohl für die Lebenswirklichkeit der Häftlinge von großer Bedeutung war. Im Unterschied zu deutschen Historikern haben polnische Historiker wie Tadeusz Szymański und Tadeusz Iwaszko dem Thema Post der Häftlinge spezielle Untersuchungen gewidmet. ${ }^{17}$ Auch der niederländische Sobibor-Überlebende Jules Schelvis geht in seinem Sobibor-Buch ausführlich und mit präzisen Informationen auf die Post der >Arbeitsjuden` (alle anderen Juden wurden sofort nach der Ankunft vergast) ein. ${ }^{18}$

Von Eugen Kogon stammt der informativste authentische Bericht eines deutschen Autors über den Postverkehr der Gefangenen. In seinem Buch Der SS-Staat schreibt er stilistisch holprig, aber inhaltlich genau und zutreffend:

"Den Briefen und Karten war ein vielzeiliger, den Schreibraum weiter verknappender Auszug aus der sogenannten Lagerordnung vorgedruckt (von der kein Mensch jemals ein Exemplar gesehen hat), derzufolge der Häftling im Lager alles kaufen konnte und daher auch Geld empfangen durfte (wieviel, war nicht angegeben, 30 Mark wurden [...] an jeden Häftling nur ausgezahlt, mit dem Rest, meist mehreren Millionen Mark, da die Angehörigen glaubten, sie könnten schicken, was sie wollten, manövrierte die SS). Den Juden war oft monatelang nicht gestattet, zu schreiben; die Angehörigen der Strafkompanien durften es nur alle Vierteljahre. Über das gesamte Lager wurde von Zeit zu Zeit aus irgendwelchen Anlässen Briefsperre verhängt. Von der ankommenden Post erhielt der Häftling häufig nur zerschnittene Schnipsel oder den leeren Umschlag, während die SS den Inhalt zerrissen hatte. Das war besonders bitter. [...] Der Buchenwalder Blockführer Kubitz kam zuweilen mit der gesamten Post für den Block 36 an, zeigte den Stoß her, verlas die einzelnen Namen und steckte das ganze Paket mit den Worten `So, ihr Schweine, nun wisst ihr, dass ihr Post bekommen habt! in den Ofen. ${ }^{19}$

Eine der wenigen Autorinnen/Autoren, die dokumentarische Quellen zum Postverkehr von KZ-Gefangenen untersucht haben, ist Gabriele Bergner. Sie hat in ihrer Dissertation über italienische Häftlinge im KZ Dachau auch über den Zugang - oder vielmehr den mangelnden Zugang - dieser Häftlingsgruppe

Stammlager (Arbeit und Vernichtung), in: Wolfgang Benz, Barbara Distel (Hrsg.): Der Ort des Terrors, Bd. 5: Hinzert, Auschwitz, Neuengamme, München 2007, S. 99-106, hier: S. 101 f.

17 Szymański: Briefe an die nächsten Angehörigen; Tadeusz Iwaszko: »Kontakt mit der Außenwelt".

18 Jules Schelvis: Vernichtungslager Sobibór, Hamburg/Münster 2013 (zuerst 1993 in den Niederlanden erschienen), S. 149.

19 Kogon: Der SS-Staat, S. 129. 
zum Postverkehr berichtet. ${ }^{20}$ Hierauf wird weiter unten, im Abschnitt Italiener, zurückzukommen sein.

Es soll nun drei Fragenkomplexen nachgegangen werden:

Erstens: Welche Veränderungen traten in den Bestimmungen zum Postverkehr der Häftlinge zwischen 1933 und 1945 ein, und lag den Veränderungen ein System zugrunde?

Zweitens: Auf was für ein Dokument bzw. auf was für Dokumente beziehen sich der in Postvordrucken angeführte "Auszug aus der Lagerordnung« bzw. der Passus »Folgende Anordnungen sind beim Schriftverkehr mit Gefangenen zu beachten«?

Drittens: Welche Ausnahmen und Einschränkungen galten zwischen 1933 und 1945 für welche Gruppen von Häftlingen?

\section{Veränderungen in den Bestimmungen zum Postverkehr 1933-1945}

Für die Qualität der Teilnahme am Postverkehr werden zwei Kriterien herangezogen: a) die Häufigkeit des Empfangs und Absendens von Briefen bzw. Postkarten, b) die Häufigkeit des Empfangs von Paketen. Eine chronologisch angeordnete Zusammenfassung der hierzu auf Vordruck-Postkarten und -Briefumschlägen der Konzentrationslager gedruckten Bestimmungen, Informationen aus dem Lørdahl-Katalog, der Himmlerschen Dienstanweisung für Konzentrationslager von 1941 (siehe weiter unten) und dem Himmler-Erlass vom 29. Oktober 1942 zur Wiederzulassung des Paketempfangs für Häftlinge (siehe weiter unten) ergeben für die Zeit vom 10. August 1933 bis 8. Mai 1945 das in der nachfolgenden Tabelle dargestellte Bild. Die Zahlen zum Brief- bzw. Postkartenverkehr beziehen sich sowohl auf die abgehende als auch auf die hereinkommende Post.

Wie man sieht, war die Zulassung von Briefpost in den Konzentrationslagern bis April 1938 weder einheitlich noch plausibel geregelt. Während in den anderen Lagern 1937/38 Absendung und Empfang von je zwei Briefen oder Karten pro Monat die Regel war, durften Gefangene in Dachau, sofern sich die SS an die geschriebene Ordnung hielt, noch am 3. November 1937 einen Brief oder eine Postkarte pro Woche empfangen und schreiben.

20 Gabriele Bergner: Aus dem Bündnis hinter den Stacheldraht. Italienische Häftlinge im KZ Dachau 1943-1945. Deportationen und Lebensbedingungen, Hamburg 2002, S. 197 ff. 
Tabelle: Erlaubter Umfang der Teilnahme am Postverkehr nach Vorschriften auf den Postformularen, der Dienstvorschrift für KL (Lagerordnung) von 1941 und dem Himmler-Erlass vom 29.10.1942

\begin{tabular}{|l|l|l|}
\hline Erlaubter Umfang & Lager & Datum \\
\hline $\begin{array}{l}\text { Im Monat ein Brief und eine Postkarte, ein Wä- } \\
\text { schepaket. }\end{array}$ & Dachau & 8.5 .1933 \\
\hline $\begin{array}{l}\text { In der Woche ein Brief und eine Postkarte, zwei- } \\
\text { mal im Monat ein Wäschepaket. }\end{array}$ & Dachau & 19.3 .1934 \\
\hline $\begin{array}{l}\text { Im Monat zwei Briefe oder zwei Postkarten, } \\
\text { zweimal im Monat ein Wäschepaket. }\end{array}$ & Sachsenburg & 18.2 .1935 \\
\hline $\begin{array}{l}\text { In der Woche ein Brief oder eine Postkarte. Pake- } \\
\text { te nicht zulässig. }\end{array}$ & Esterwegen & 16.11 .1935 \\
\hline $\begin{array}{l}\text { Im Monat zwei Briefe oder zwei Postkarten. } \\
\text { Pakete nicht zulässig gem. handschr. Korrektur. }\end{array}$ & Columbia & 7.10 .1936 \\
\hline Im Monat zwei Briefe oder zwei Postkarten. & Lichtenburg & 14.3 .1937 \\
\hline $\begin{array}{l}\text { Im Monat zwei Briefe oder zwei Postkarten. } \\
\text { Pakete nicht zugelassen. }\end{array}$ & Buchenwald & 5.5 .1937 \\
\hline $\begin{array}{l}\text { In der Woche ein Brief oder eine Postkarte. Pake- } \\
\text { te nicht zugelassen. }\end{array}$ & Dachau & 3.11 .1937 \\
\hline $\begin{array}{l}\text { Im Monat zwei Briefe oder zwei Postkarten. } \\
\text { Pakete nicht zugelassen. }\end{array}$ & Sachsenhausen & 13.4 .1938 \\
\hline $\begin{array}{l}\text { Im Monat zwei Briefe oder zwei Postkarten. } \\
\text { Pakete nicht zugelassen. }\end{array}$ & Dachau & Alle Lager \\
\hline $\begin{array}{l}\text { Im Monat zwei Briefe oder zwei Postkarten. } \\
\text { Pakete nicht zugelassen. }\end{array}$ & Ravensbrück & Ab 1941 \\
\hline $\begin{array}{l}\text { Im Monat ein Brief oder eine Karte. Pakete nicht } \\
\text { zugelassen. }\end{array}$ & Ravensbrück & Ab Januar 1941 \\
\hline $\begin{array}{l}\text { Im Monat zwei Briefe oder zwei } \\
\text { Postkarten. Pakete nicht zugelassen. }\end{array}$ & Alle Lager \\
\hline Lebensmittelpakete zugelassen. & Ab 29.10 .1942 \\
\hline
\end{tabular}

In dem untersuchten Material taucht auf einer Postkarte aus dem Konzentrationslager Columbia vom 7. Oktober 1936 erstmals die Regelung auf, nach der monatlich zwei Briefe oder zwei Postkarten und keine Pakete zugelassen waren. ${ }^{21}$ Die Vorschrift, nach der monatlich der Empfang und das Absenden

21 Im gedruckten Text ist noch die Rede von zwei Wäschepaketen pro Monat. Im geschriebenen Text hat der Absender aber, zweifellos auf Veranlassung der SS-Zensur, seine Bitte um Wäsche durchgestrichen und in die Bitte umgewandelt, ihm keine Wäsche zu schicken. 
von je zwei Briefen oder Postkarten zugelassen war, wurde schrittweise in den anderen Lagern eingeführt und galt mit vielen faktischen Ausnahmen bis zum Zusammenbruch des Lagersystems. Die einzige institutionelle Ausnahme war, soweit bekannt, das Frauen-Konzentrationslager Ravensbrück, wo Häftlinge ab 1941 nur je einen Brief oder eine Karte pro Monat absenden und empfangen durften.

Die eingangs gestellte Frage, ob den Veränderungen in den Bedingungen zum Postverkehr der Häftlinge ein System zugrunde lag, lässt sich nicht schlüssig beantworten.

Wie der Tabelle zu entnehmen ist, war in den ersten zwei bis drei Jahren der Empfang von Wäschepaketen zugelassen. Die Erlaubnis, Wäschepakete zu empfangen, ist zum letzten Mal für die dreißiger Jahre auf einem Faltbrief vom 18. Februar 1935 aus dem Lager Sachsenburg nachgewiesen.

Das erste Zeugnis der Nichtzulassung von Paketen ist die erwähnte Postkarte aus dem Lager Columbia vom 7. Oktober 1936. Die erste ausdrückliche Bestimmung »Pakete jeglichen Inhalts dürfen nicht empfangen werden « findet sich auf einer Postkarte aus dem KL Esterwegen vom 16. November 1935. Ähnliche Formulierungen sind dann in den Anweisungen aller Lager bis Ende Oktober 1942 enthalten.

Aufgrund des Himmlerschen Erlasses vom 29. Oktober 1942 über die Zulassung des Empfangs von Lebensmittelpaketen ${ }^{22}$ wurde zunächst in den Vordrucken das Wörtchen "nicht« in dem Satz »Pakete jeglichen Inhalts dürfen nicht empfangen werden" handschriftlich durchgestrichen wie in Abbildung 4, einem Umschlag aus dem KL Ravensbrück vom 16. August 1943. Später enthielten die Vorschriften Sätze, die geradezu nach einer Bitte klangen, wie diesen: »Lebensmittelpakete dürfen zu jeder Zeit und in jeder Menge empfangen werden." (Abbildung 5). Die Ursache für die Wende in Bezug auf Paketempfang war nicht etwa eine humane Regung Himmlers, sondern das Bestreben der SS, die Häftlinge, die jetzt für die Kriegsindustrie benötigt wurden, auf Kosten ihrer Freunde und Verwandten sowie internationaler Hilfsorganisationen ein wenig länger für die Zwangsarbeit bei Kräften zu halten.

Die zunächst etwas privilegierte Gruppe der >Protektoratshäftlinge` (erkennbar an der Benutzung neutraler Postkarten und Briefumschläge) durfte offenbar in der ersten Zeit ihrer Haft in Buchenwald Pakete mit Pullovern und Unterwäsche empfangen, wie dem Nebenstempel »In Paketen sind nur Pullower und/Unterwäsche zugelassen, alles an-/dere verfällt der Beschlagnahme!« auf der Postkarte, Poststempel Weimar 1, 23. Oktober 1939 (Abbildung 6), zu entnehmen ist. Auch diese Häftlinge unterlagen wenig später dem vollständi-

22 Reichsführer SS und Chef der Deutschen Polizei: Paketsendungen an Häftlinge, 29. Oktober 1942. Quelle: Bundesarchiv (im Folgenden: BArch) NS 4/NA 3, Bl. 5. 
gen Verbot des Paketempfangs, wie aus dem Nebenstempel »Paketsendungen jeder Art/VERBOTEN« auf der Postkarte, Poststempel Weimar 1, 17. Januar 1940, (Abbildung 7) hervorgeht.

Von Fall zu Fall wurde es den Kommandanten freigestellt, zu Ostern, Pfingsten oder Weihnachten den Paketempfang zu gestatten. Wenn sie diese Möglichkeit den Häftlingen vorenthielten, wurde dies auf Beilagezetteln mitgeteilt, die die Gefangenen ihren Briefpartnern zu senden hatten.

Durch einen Erlass des Reichsführers SS vom 24. September 1941 wurde das Verbot des Paket-Empfangs mit zeitlicher Begrenzung etwas gelockert. ${ }^{23}$ Himmler erteilte hier die Genehmigung für die Zusendung von »Wäschepaketen (Hemden, Unterhosen, Pullover) für die bevorstehende kalte Jahreszeit«. Die Kommandanten der Konzentrationslager setzten die Genehmigung um, indem sie entsprechende Mitteilungen auf Beilagezettel drucken ließen, die die Häftlinge ihren Briefen beilegen konnten.

Nach dem Himmlerschen Erlass vom 29. Oktober 1942 war die Genehmigung zum Paketempfang auf Lebensmittelpakete beschränkt, später (das Datum ist nicht bekannt) wurde sie auf Pakete jeglichen Inhalts erweitert. »Diese Vergünstigung«, schreibt Skriebeleit mit Bezug auf den Erlass vom 29. Oktober 1942, »kam aber lediglich deutschen, ab Januar 1943 dann auch tschechischen und westeuropäischen Häftlingen zugute. « ${ }^{24}$ Spätestens im Mai 1943 durften auch polnische Häftlinge Pakete empfangen, wie die Paketkarte in Abbildung 8 für ein Paket aus Reichshof (Rzeszow) im Generalgouvernement belegt.

Sowjetische Häftlinge und Juden blieben vom Paketempfang ausgeschlossen. Im Falle der sowjetischen Häftlinge wurde das Verbot des Paketempfangs strikt eingehalten. Für jüdische Häftlinge gab es nach Kenntnis des Autors zwei oder drei Ausnahmen. Die eine betraf die Insassen des Theresienstädter Familienlagers Auschwitz-Birkenau. Überlebende haben berichtet, dass einige Gefangene dieses Lagers tatsächlich Pakete bekommen haben. ${ }^{25}$ Die andere Ausnahme betraf die Mitglieder des Fälscherkommandos Sachsenhausen. ${ }^{26}$ Eine weitere Ausnahme waren die aus dem Familienlager Auschwitz stammenden Häftlinge des Sachsenhausener Außenlagers Schwarzheide. Jedenfalls teilten

23 Inspekteur der Konzentrationslager: Übersendung von Unterwäsche für Häftlinge, 24. September 1941. Quelle: BArch NS/425, Bl. 34.

24 Jörg Skriebeleit: »Flossenbürg - Stammlager«, in: Benz, Distel (Hrsg.): Der Ort des Terrors, Bd. 4: Flossenbürg, Mauthausen, Ravensbrück, München 2007, S. 17-66, hier: S. 43.

25 So die Überlebende Dagmar Lieblová in Gesprächen mit dem Verfasser. Zum Thema Paketsendungen in das Theresienstädter Familienlager Auschwitz-Birkenau vgl. auch »Das Theresienstädter Familienlager Auschwitz-Birkenau«, in: Heinz Wewer: Postalische Zeugnisse zur deutschen Besatzungsherrschaft im Protektorat Böhmen und Mähren, 2., durchges. Aufl., Berlin 2018, S. $167 \mathrm{ff}$.

26 Zum Fälscherkommando vgl. Heinz Wewer: Spuren des Terrors. Postalische Zeugnisse zum System der deutschen Konzentrationslager, Berlin/Leipzig 2020, S. 77 f. 
sie ihren Angehörigen mit, dass sie Pakete empfangen dürften, und es sind auch Einlieferungsbestätigungen überliefert. ${ }^{27} \mathrm{Ob}$ sie tatsächlich Pakete erhalten haben, ist nicht bekannt.

Pakete, die nicht zugestellt werden konnten, weil die Adressaten nicht mehr lebten oder sauf Transport ' geschickt worden waren, behielt die SS für sich. Es kam offenbar nicht selten vor, dass die SS auch die in großer Zahl eintreffenden Pakete des Roten Kreuzes und Geldsendungen unterschlug. ${ }^{28}$ Ein Häftling, der von Januar 1943 bis zum Herbst 1944 in der Poststelle Buchenwald arbeitete, berichtet:

»In der Paketpoststelle waren die für die Häftlinge bestimmten Pakete Freigut für die SSRäuber. Systematisch wurden die Pakete ausgeplündert, was die SS-Leute gebrauchen konnten, nahmen sie sich aus den Paketen heraus. Das Diebesgut schickten die Banditen nach Hause, die Lebensmittel aßen sie selbst oder verwerteten das gestohlene Gut als Tauschmittel. « ${ }^{29}$

\section{Die Besonderen Lagerordnungen für Esterwegen und Lichtenburg}

Wie gezeigt, waren die gedruckten Bestimmungen für den Postverkehr der Gefangenen in den ersten Jahren mit der Zeile »Auszug aus der Lagerordnung " überschrieben. Diese Zeile scheint in Dachau zum letzten Mal auf einer Postkarte mit Poststempel vom Januar 1936 in Gebrauch gewesen zu sein. ${ }^{30} \mathrm{Da}$ nach wurde sie durch die Formel »Folgende Anordnungen sind im Schriftverkehr mit Gefangenen zu beachten« ersetzt. Es gab Lager, in denen die alte Formel weiterverwendet und solche, in denen die neue benutzt wurde. Ein System in der unterschiedlichen Anwendung der beiden Formeln konnte der Autor nicht entdecken.

Es bleibt die Frage, was für eine »Lagerordnung « das war, von der angebliche Auszüge auf vielen Postvordrucken gedruckt waren. Die berüchtigte Eickesche Disziplinar- und Strafordnung vom 1. Oktober 1933, zuerst in Dachau und dann in allen anderen Lagern erlassen, konnte nicht gemeint gewesen sein, denn hier war nicht von allgemeinen Bestimmungen zum Postverkehr die Rede, sondern lediglich von Postsperre als einer von mehreren möglichen Strafen.

27 Zum Außenlager Schwarzheide vgl. ebenda, S. 85 f.

28 Kogon: Der SS-Staat, S. $123 \mathrm{ff}$.

29 Alfons Marincovich: »SS-Leute als Posträuber«, in: Hackett (Hrsg.) Der Buchenwald-Report, S. 183.

30 Lørdahl: German Concentration Camps, Bd. II, Teil 1, S. 171. 
Detaillierte Bestimmungen zum Postverkehr der Häftlinge sind jedoch in zwei anderen, bislang nach Kenntnis des Autors in der wissenschaftlichen Literatur zur Geschichte der Konzentrationslager wenig beachteten Dokumenten überliefert, nämlich der Besonderen Lagerordnung für das GefangenenBarackenlager, erlassen für das Konzentrationslager Lichtenburg, datiert vom 1. Juni 1934 und in einer Ordnung mit derselben Bezeichnung, erlassen für das Konzentrationslager Esterwegen am 1. August 1934.

Die beiden Ordnungen unterscheiden sich nur unwesentlich voneinander. So heißen die Sammelunterkünfte der Häftlinge in der Lichtenburg-Ordnung "Gemeinschaftsstuben", in der Esterwegen-Ordnung heißen sie "Baracken" (jeweils Punkt 6). Die ältere der beiden Ordnungen, die für Lichtenburg, ist nach Kenntnis des Autors bisher nicht veröffentlicht. Sie wurde daher im Anhang des Buches, aus dem die Urfassung dieses Beitrages stammt, vollständig faksimiliert. ${ }^{31}$ Die Esterwegen-Ordnung ist an einem nicht leicht zugänglichen Ort (innerhalb einer dreibändigen Dokumentation von insgesamt 3630 Seiten) publiziert worden. ${ }^{32}$

31 Wewer: Spuren des Terrors, S. 284 ff. Die »Besondere Lagerordnung für das GefangenenBarackenlager « für das Konzentrationslager Lichtenburg wird im Bundesarchiv Berlin unter der Signatur RY 1/I/2/3/158, S. 319-326 geführt. Das Dokument stammt aus dem Archiv des Politbüros der KPD und kam über das Zentralarchiv der SED mit dem Zentralen Staatsarchiv der DDR an das Bundesarchiv. Die Vermutung liegt nahe, dass das Exemplar von der kommunistischen Parteiorganisation im Lager Lichtenburg vor der Vernichtung bewahrt wurde und sodann ins Archiv der KPD gelangt ist. In der historischen Forschung scheint die »Besondere Lagerordnung für das Gefangenen-Barackenlager« für Lichtenburg unbekannt zu sein. Stefanie Endlich erwähnt in ihrem Beitrag "Lichtenburg«, in: Benz, Distel (Hrsg.): Der Ort des Terrors, Bd. 2: Frühe Lager, Dachau, Emslandlager, München 2005, S. 151-159, hier: S. 153 lediglich die "Dachauer Lagerordnung", womit sie offensichtlich die Eickesche »Disziplinar- und Strafordnung« meint. Die »Besondere Lagerordnung für das Gefangenen-Barackenlager« für Lichtenburg erwähnt sie nicht.

32 Erich Kosthorst, Bernd Walter (Hrsg.): Konzentrations- und Strafgefangenenlager im Dritten Reich. Beispiel Emsland, Band 1, Düsseldorf 1983, S. 197 ff. Die Herausgeber nennen als Quelle IfZ, Fa 253. Die Esterwegen-Ordnung ist im Internet-Portal Gelsenzentrum mit Datum vom Mai 2008 reproduziert. Von der »Besonderen Lagerordnung für das Gefangenen-Barackenlager « für das Konzentrationslager Esterwegen sind zwei weitere Exemplare bekannt. Sie werden im Bundesarchiv Berlin unter den Signaturen N 2001/51, S. 3-12 bzw. NS 4 ANH aufbewahrt. Die »Besondere Lagerordnung für das Gefangenen-Barackenlager» für Esterwegen wird nach Kenntnis des Autors in geschichtswissenschaftlichen Untersuchungen nur ein einziges Mal erwähnt, und zwar in einer gekürzten Fassung, in der der Name des »F. d. R. « zeichnenden Adjutanten Weibrecht weggelassen ist. Diese findet sich bei Erich Kosthorst, Bernd Walter: Konzentrations- und Strafgefangenenlager im Emsland 1933-1945. Zum Verhältnis von NS-Regime und Justiz, Düsseldorf 1985, S. 85 ff. In dem Beitrag von Habbo Knoch: Die Emslandlager 1933-1945, in: Benz, Distel (Hrsg.): Der Ort des Terrors, Bd. 2, S. 533-570 wird zwar auf S. 541 eine »Lager- und Disziplinarordnung « für das KZ Esterwegen nach Dachauer Vorbild erwähnt, dass es daneben noch die »Besondere Lagerordnung für das GefangenenBarackenlager« vom 1. August 1934 gab, erfährt man jedoch nicht. 
Schon ein erster Blick zeigt, dass es sich bei den Bestimmungen zur Lagerpost in den beiden Besonderen Lagerordnungen um jene Bestimmungen handeln muss, die den auf den Postvordrucken der Konzentrationslager aufgedruckten »Auszügen aus der Lagerordnung « und entsprechenden Passus zugrunde liegen. Dem Häftling ist es hiernach erlaubt, pro Monat zwei Briefe oder zwei Postkarten von seinen Angehörigen zu empfangen oder an diese abzusenden. Die Grenzen des inhaltlich Erlaubten werden eng gezogen:

»Wer in einem Brief abfällige Bemerkungen über Staat und Regierung, Behörden und Einrichtungen zum Ausdruck bringt, oder marxistische oder liberalistische Führer oder Parteien verherrlicht, Vorgänge im Konzentrationslager mitteilt, gilt als unverbesserlich und wird zur Verantwortung gezogen.«

Dies waren Vorschriften, die den Gefangenen offenbar nicht mitgeteilt wurden - so das Zeugnis Kogons ${ }^{33}$-, die sie daher aus der Praxis erlernen mussten. Die in den beiden Ordnungen vorgesehene Möglichkeit zum Empfang von Paketen wurde, wie dargelegt, etwa im Oktober 1936 gestrichen und erst durch den Erlass Himmlers im Oktober 1942 wieder eingeführt.

Interessant in den beiden Dokumenten ist die Beschreibung der Haftstufen I (Entlassungsstufe) und III (schwere Fälle). Die dazwischenliegende Stufe II, die `Normalstufe`, wird gar nicht erst beschrieben. In welche Stufe ein Häftling eingeordnet wurde, blieb diesem natürlich verborgen, wurde aber in die Häftlingsakte eingetragen. Die Aufzählung der >Tatbestandsmerkmale` für die Einstufung in die Stufe III zeigt exemplarisch, welchen praktisch unbegrenzten Spielraum die Lager-SS bei der Terrorisierung der Häftlinge hatte. In der Lichtenburg-Ordnung heißt es unter Punkt 9. B.):

»In die Stufe III werden versetzt:

a.) Leitende politische Persönlichkeiten und Intellektuelle, deren bisheriges oder früheres Wirken als besonders verderblich für Volk und Staat anerkannt wurde, [...]

e.) Juden und andere Personen, welche sich als Volksschädlinge oder als gemeine politische Hetzer bemerkbar gemacht haben, $[\ldots]$

g.) Gefangene, die in ihren Briefen zu erkennen geben, dass sie sich absolut nicht umstellen wollen.«

Später erfolgte eine entsprechende Einstufung auch der Konzentrationslager. Hiernach waren in der Stufe I: Dachau, Sachsenhausen und Auschwitz I; in der

33 Kogon: Der SS-Staat, S. 129. 
Stufe II: Buchenwald, Flossenbürg, Neuengamme, Natzweiler, Stutthof und Auschwitz II (Birkenau); in der Stufe III: Mauthausen und Groß-Rosen. ${ }^{34}$

Die Dokumente tragen ein maschinenschriftliches "gez. Eicke« und die Unterschrift von Eickes Adjutanten Hans Weibrecht unter der Formel »F.d.R.« (Für die Richtigkeit). Daher ist zu vermuten, dass es sich bei den beiden überlieferten Fassungen um Abschriften vom Original, also sehr wahrscheinlich von der Dachauer Urfassung, handelt. Wo aber ist das Original? Die Nachforschungen nach einer Dachauer oder einer sonstigen Urfassung, die der Autor bei zahlreichen Archiven, darunter dem Archiv der KZ-Gedenkstätte Dach$\mathrm{au},{ }^{35}$ angestellt hat, sind ohne Ergebnis geblieben.

\section{Himmlers Dienstvorschrift für Konzentrationslager (Lagerordnung) von 1941}

In der Fachliteratur wird die Meinung vertreten, die Eickesche Lagerordnung habe mit geringfügigen Änderungen bis in die Kriegsjahre hinein gegolten. ${ }^{36}$ Diese Auffassung lässt sich nicht halten. Denn die vom Autor mit Hilfe einer aufmerksamen Archivarin der Mahn- und Gedenkstätte Ravensbrück aufgefundene Dienstvorschrift für Konzentrationslager (Lagerordnung), Unterschrift H. Himmler, ist 1941 an die Stelle der Eickeschen Ordnung getreten und galt bis zum Ende des NS-Regimes. ${ }^{37}$ Die Eickesche Strafordnung ist nur ein kleiner Bestandteil der Dienstvorschrift. Gedruckt wurde das Dokument im Reichssicherheitshauptamt, Berlin 1941. Die Dienstvorschrift wurde in nummerierten Exemplaren an den Inspekteur der Konzentrationslager, die Kom-

34 Verfügung Liebehenschel, Stabsführer der Inspektion der Konzentrationslager, o. D. Quelle: BArch, NS 4/Buchenwald 32. Zur Einstufung der Konzentrationslager vgl. auch Wewer: Spuren des Terrors, S. $22 \mathrm{f}$.

35 E-Mail des Archivleiters der KZ-Gedenkstätte Dachau, Albert Knoll, an den Autor vom 3. Juli 2012.

36 Johannes Tuchel: Die Inspektion der Konzentrationslager 1938-1945. Das System des Terrors, Berlin 1994, S. 100; ders.: Nationalsozialistische Herrschaftssicherung und Verfolgungspraxis 1933 bis 1937, in: Volkhard Knigge u. a. (Hrsg.): Buchenwald Ausgrenzung und Gewalt 1937 bis 1945, Ausstellungsbegleitbuch, Göttingen 2016, S. 236-243, hier: S. 239; Nikolaus Wachsmann: kl, Die Geschichte der nationalsozialistischen Konzentrationslager, München 2016, S. 231 und 787.

37 M.W. ist die Himmlersche Lagerordnung von 1941 in der wissenschaftlichen Literatur bisher nur ein einziges Mal erwähnt worden, und zwar im Abschnitt »Der Postenbegleitdienst« in: Johannes Tuchel: Die Wachmannschaften der Konzentrationslager 1939 bis 1945 - Ergebnisse und offene Fragen der Forschung, in: Alfred Gottwaldt, Norbert Kampe, Peter Klein (Hrsg.): NS-Gewaltherrschaft. Beiträge zur historischen Forschung und juristischen Aufarbeitung, Berlin 2005, S. 135-151, hier: S. 146. In der zugehörigen Anmerkung 73 ist der Aufbewahrungsort des Dokumentes nicht angegeben. 
mandanten und möglicherweise weitere Institutionen/Personen verteilt. Das einzige bisher bekannte Original befindet sich im Staatsarchiv der Russischen Föderation (GARF) in Moskau. ${ }^{38}$

Die Dienstvorschrift umfasst 50 gedruckte Seiten. Hiervon nimmt die Strafordnung knapp fünf Seiten ein. Die Kapitel I bis X sind eine Darstellung der Struktur und der Funktionen des Lagers und seiner Bestandteile aus Sicht der SS. Kapitel XI regelt »Aufnahmen, Entlassungen und Verschubungen [sic] «, Kapitel XII die »Häftlingsgeldverwaltung« und Kapitel XIII die »Postzensur«. Die Kapitel XIV »Allgemeine Lagerordnung « und XV »Strafordnung "sind so etwas wie eine Kombination von Elementen der Eickeschen Straf- und Disziplinarordnung mit der (vermutlich ebenfalls auf Eicke zurückgehenden) Besonderen Lagerordnung.

Hinsichtlich des Postverkehrs der Häftlinge entspricht das Kapitel XIII »Die Postzensur « dem Stand, wie er sich etwa Ende 1939 aus den Bestimmungen ergibt, die auf den Formblättern der Häftlingspost gedruckt sind: »Jeder Häftling darf im Monat zwei Briefe oder zwei Karten schreiben oder empfangen." (Punkt 2, Satz 2) und »Der Empfang von Paketen und Päckchen ist den Häftlingen verboten...« (Punkt 11, erster Halbsatz). Das Kapitel XIII ist im Wesentlichen eine Art Büroordnung, die penibel beschreibt, wie mit der Häftlingspost, der hereinkommenden und der hinausgehenden, zu verfahren ist, vor allem wie die Zensur zu handhaben ist. Die Betonung des Kapitels liegt, wie die Überschrift zutreffend angibt, nicht auf Post der Häftlinge, sondern auf Zensur.

Die Dienstvorschrift enthält bizarre Anweisungen über die Verhinderung von Nachrichtenschmuggel durch Kontrolle der Briefmarken, die sich auf der eingehenden Häftlingspost befinden: »Zur Verhinderung des Nachrichtenschmuggels, durch Benutzung des Raumes unter den Marken, sind nach Zensur der Postsendungen die Freimarken auszuschneiden« (Punkt 4, Satz 2). ${ }^{39}$ Und: »Die ausgehende Post ist von den Häftlingen den zuständigen Blockführern persönlich und offen zu übergeben. Die Marke darf zur Verhinderung verbotener Mitteilungen nur mit einer Ecke aufgeklebt sein." (Punkt 7, Satz 1 und 2.) "Nach der Zensur werden die Briefe verschlossen, die Marken voll aufgeklebt und die versandfertige Post befördert.« (Punkt 7, Satz 5.)

38 H. Himmler, Dienstvorschrift für Konzentrationslager (Lagerordnung) 1941. Quelle: Staatsarchiv der Russischen Föderation (GARF), Moskau, Bestand 7021, Vorgang 1, Akte 92, Bl. 9. Titelblatt und Kapitel XIII reproduziert in: Wewer, Spuren des Terrors, S. 292-296.

39 Die Vorschrift hat einen Vorläufer. In einer Verfügung des Inspekteurs der Konzentrationslager Eicke an die Kommandanten der Konzentrationslager vom 3. Februar 1937 heißt es: 1 . Um Nachrichtenschmuggel durch Benutzung des Raumes unter den Freimarken zu verhindern, sind von der Gefangenen-Eingangspost die aufgeklebten Freimarken auszuschneiden...« Quelle: Copy of Doc. No. 82351920\#1 (11.1.20.0/0002/0094) in conformity with ITS Archives. 
Unter den überlieferten, nicht sehr zahlreichen Postsendungen an Häftlinge in Konzentrationslagern finden sich etliche, bei denen die Briefmarke ausgeschnitten ist. Es ist jedoch nicht festzustellen, mit welcher Motivation dies geschehen ist - ob aufgrund der Vorschriften des RSHA, aus Sammlerinteresse, aus Aversion gegen den Kopf Hitlers oder weil Briefmarken der NS-Zeit offiziell unerwünscht waren wie in der DDR. Das Fehlen von Briefmarken auf ausgehender Post ist jedenfalls prinzipiell nicht auf die Zensur zurückzuführen. Denn die Post ging ja ohne aufgeklebte Briefmarke an die Zensurstelle, sie wurde dort zensiert, und erst danach wurde die Briefmarke aufgeklebt, und die Sendung wurde von der SS zur Post gegeben. Die Briefmarke kann sich daher erst nach der Aufgabe bei der Post gelöst haben, oder jemand löste sie ab, jedenfalls nicht die Zensur.

Eine Kopie der Dienstvorschrift wird im Archiv der Mahn- und Gedenkstätte Ravensbrück aufbewahrt. ${ }^{40}$ Der Unterschied zwischen den beiden Fassungen fällt sofort in die Augen. Während die Ravensbrücker Fassung mit dem Dienstsiegel des Inspekteurs der Konzentrationslager und der Nummer 005 versehen ist, befindet sich auf dem Moskauer Dokument kein Dienstsiegel, und eine etwa vorhandene Nummer ist durch einen archivalischen Notizzettel (oben rechts auf dem Titelblatt) verdeckt. Ein Vergleich der Schriftart, des Schriftbildes und vor allem der auf beiden Dokumenten in charakteristischer Handschrift aufgetragenen Foliierung (fortlaufende Nummerierung der Blätter) 27 und 28 zeigt aber, dass es sich um ein und dasselbe Dokument handelt mit dem Unterschied, dass auf dem Moskauer Original das Dienstsiegel entfernt worden ist. Es ergibt sich also die absonderliche Situation, dass die Kopie vollständiger ist als das Original. Betrachten wir nun das vom sowjetischen Anklagevertreter im Nürnberger Hauptkriegsverbrecherprozess als maschinenschriftliches Typoscript vorgelegte Dokument 011-USSR mit dem Titel "Dienstvorschrift für Konzentrationslager ${ }^{41}{ }^{4}$ Hier befindet sich auf der ersten Seite rechts oben der gedruckte Vermerk »Nr. 005«. Also ergibt sich der zwingende Schluss, dass es sich bei den Dienstvorschrift-Fassungen der Quellen Moskau, Ravensbrück und Nürnberg um ein- und dasselbe Dokument in unterschiedlichen Fassungen handelt.

40 H. Himmler, Dienstvorschrift für Konzentrationslager (Lagerordnung) 1941. Quelle: Mahnund Gedenkstätte Ravensbrück, Archiv, SARF/3-1. Titelblatt und Kapitel XIII sind reproduziert in: Wewer, Spuren des Terrors, S. 297-301. Ich danke der Archivarin der Mahn- und Gedenkstätte Ravensbrück, Monika Schnell, dass sie mich auf das Dokument aufmerksam gemacht und damit meine Suche nach dem Moskauer Original in Gang gesetzt hat.

41 IMT, Bd. 39, S. 262 ff. 
Der in Ravensbrück aufbewahrten Fassung der Dienstvorschrift sind Anlagen beigefügt, die als Kapitel XVI listenmäßig aufgeführt sind:

»Muster 1: Benachrichtigungen für die Häftlings-Angehörigenfürsorge

Muster 2 (Tafel): Kennzeichen für Schutzhäftlinge in den Konzentrationslagern

Muster 3 - 5: Strafverfügungen

Muster 6: Vorgeschriebenes Briefpapier für Häftlinge (1. Und 4. Seite)

Muster 7: Briefumschlag und Postkarte für Häftlinge«.

Die Herkunft und der Weg des Dokuments lassen sich bisher nicht schlüssig aufklären. Denkbar ist der folgende Gang der Dinge: Bei der Besetzung der Dienststelle des Inspekteurs der Konzentrationslager in Oranienburg hat die Rote Armee die dort vorgefundene Dienstvorschrift beschlagnahmt. Danach hat der sowjetische Hauptankläger sie in den Nürnberger Hauptkriegsverbrecher-Prozess eingeführt. Anschließend wurde das Dokument nach Moskau transportiert und dem damaligen Staatlichen Archiv der UdSSR übergeben. Dieses hat die Blätter (nicht die Seiten) foliiert und die nunmehrige Archivalie mit einer Signatur versehen. Von dem so beschaffenen Dokument gelangte eine Kopie auf einem bisher nicht bekannten Weg in das Archiv der Mahnund Gedenkstätte Ravensbrück. ${ }^{42}$ Irgendwann nach der Abgabe der Kopie hat jemand in dem Moskauer Archiv vom Original das Dienstsiegel entfernt, ob aus eigener Initiative oder als Teil einer `Säuberung ‘ der Akten von `Symbolen des Faschismus`, lässt sich nicht feststellen.

Die Analyse der Moskauer Archivalie und die Umstände ihrer Verlagerung in die UdSSR lassen mindestens zwei Fragen als klärungsbedürftig erscheinen:

1. Das Dokument beginnt mit Blatt 9 - was ist der Inhalt der Blätter 1 bis 8 ?

2. Welche anderen, möglicherweise bis heute nicht publizierten Dokumente hat die Rote Armee beim Inspekteur der Konzentrationslager oder in anderen Dienststellen beschlagnahmt und dem Archiv der damaligen UdSSR übergeben?

\section{Der Entwurf einer Lagerordnung für Ravensbrück}

Als weitere Lagerordnung mit einem Kapitel über Häftlingspost bzw. Zensur ist die Dienstvorschrift für das Fr. K. L. - Ravensbrück (Lagerordnung), ohne erkennbares Datum, überliefert. Eine Kopie des Dokuments befindet sich beim

42 Die Provenienz des Ravensbrücker Dokuments lässt sich nach Auskunft der Leiterin des Archivs der Gedenkstätte Ravensbrück nicht rekonstruieren. 
Internationalen Suchdienst IST in Bad Arolsen. ${ }^{43}$ Diese Dienstvorschrift, die allerdings oberhalb des Titels den handschriftlichen Zusatz »Vorläufige« trägt, ist offenbar eine Umsetzung oder der unvollständige Entwurf einer Umsetzung der Dienstvorschrift des RSHA. Sie ist mit jener bis auf einige Abweichungen identisch. Allerdings gibt es Redundanzen (von Post der Häftlinge ist an zwei verschiedenen Stellen jeweils ausführlich und wortgleich die Rede), und am Schluss bricht der Text unvermittelt ab.

Die Unterschiede zwischen der offenbar im Entwurfsstadium stecken gebliebenen Dienstvorschrift des KL Ravensbrück und der Himmlers sind nicht substanziell. Der Kommandant ließ sich in Ravensbrück, wie auch aus den Postvordrucken $\mathrm{zu}$ entnehmen, als »Lagerdirektor« bezeichnen. Im Übrigen spiegelt die Sprache wider, dass ein Teil des Bewachungspersonals weiblich war. Die einfachen SS-Frauen heißen »Aufseherinnen«, in der Küche fungiert nicht ein Küchenleiter, sondern eine »Küchenleiterin«. Statt eines Blockführers gibt es eine »Blockführerin« oder »Blockleiterin«. Dem Schutzhaftlagerführer wird eine »Oberaufseherin « an die Seite gestellt, die aber diesem untergeordnet ist. Es wird streng darauf geachtet, dass die SS-Frauen keine leitenden Funktionen einnehmen, sondern stets, der SS-Ideologie folgend, weisungsgebunden gegenüber den SS-Männern sind.

Dass es sich bei der Mehrheit der Gefangenen um Frauen handelte, ist hauptsächlich an Banalitäten festgemacht wie der, dass die Gefangenen ihre Haare zu Knoten zusammenzubinden haben. Nach der Lagerordnung ist es verboten, Häftlinge zu misshandeln. Zugleich werden aber die brutalsten Misshandlungen im Katalog der Strafen aufgeführt wie lichtloser Stehbunker bei Wasser und Brot oder 25 Stockhiebe, die mehrmals "verabreicht« werden können, was bekanntlich in der Regel zum Tode führt. Außerdem stand es dem SS-Personal jederzeit frei und wurde mit Sonderurlaub belohnt, Häftlinge »auf der Flucht « zu erschießen.

An einem Punkt wird besonders deutlich, wie wenig die Bestimmungen solcher Lagerordnungen in der Praxis wert waren. Ravensbrück-Häftlinge durften, darauf wurde mehrfach hingewiesen, im Gegensatz zu den Häftlingen aller anderen Lager mit Ausnahme von Lublin-Majdanek, nur einen Brief oder eine Postkarte pro Monat absenden und empfangen. In der Ravensbrücker Lagerordnung steht aber in Kapitel XIII. »Die Postzensur«: »Jeder Häftling darf im Monat zwei Brief [sic] oder zwei Karten schreiben und empfangen." (Absatz 2, Satz 2.) Der sprachliche Fehler in diesem Satz lässt sich vielleicht als Freudsche Fehlleistung deuten: Dem Schreiber war zuerst der Singular »Brief«, also die

43 Quelle: Copy of Doc. No. 82150207\#1 (1.1.35.0/0005/I438/0007) in conformity with the ITS Archives. Das Original trägt englischsprachige Vermerke. Es dürfte sich im Nationalarchiv der USA befinden. 
Wahrheit, in die Feder gerutscht, dann hat er sich eines anderen besonnen und beschlossen zu lügen, hat es aber in der Eile versäumt, die Spuren seines Abweges in die Wahrheit zu tilgen.

\section{Einschränkungen, Ausnahmen, Sonderregelungen}

Die formalen Regeln zum Brief- und Paketverkehr der Gefangenen stellten einen Rahmen dar, den die SS einhielt oder auch nicht. Aus ihnen ergab sich kein Rechtsanspruch der Gefangenen (sie hatten überhaupt keine Rechte), und die Wirklichkeit bildeten sie nur zu einem gewissen Grad ab. Der Reichsführer SS konnte jederzeit Einschränkungen, Ausnahmen und Sonderregelungen verfügen, die Lager-SS konnte aus eigener Machtvollkommenheit die Regeln ändern, die gesamte Lagerpopulation oder einzelne Häftlingsgruppen vom Postverkehr ausschließen, Post vorenthalten oder nicht weiterleiten oder Postsperren verhängen.

Bei der Bearbeitung dieses Themas werden die Lücken in den Quellen besonders spürbar. In zahlreichen Dokumenten finden sich Hinweise auf Vorgänge und/oder einzelne Dokumente, die nicht überliefert sind. Relevante Sachverhalte müssen daher oftmals aus anderen Quellen, etwa den Berichten Überlebender, erschlossen werden. Es ist zu hoffen, dass aus Archiven, insbesondere denen der Siegermächte des Zweiten Weltkrieges, noch viele Dokumente auftauchen, die unserer Kenntnis über die Geschichte der NS-Diktatur, speziell die Geschichte der postalischen Verhältnisse, zugute kommen.

\subsection{Postsperren}

Angesichts der großen Bedeutung des Postverkehrs für zumindest einen erheblichen Teil der Häftlinge war die Einschränkung der Teilnahme am Postverkehr und die Verhängung von Postsperre für die SS ein probates Mittel der Bestrafung und Disziplinierung. Die Einschränkung des Postverkehrs konnte für einzelne Häftlinge angeordnet werden. So durfte der tschechische Häftling Leopold Pliczka nicht, wie üblich, zwei Postsendungen pro Monat empfangen und absenden, sondern nur einen Brief (Abbildung 9), und der polnische Häftling Tadeusz Koszyka durfte gar nur alle sechs Wochen einen Brief empfangen und schreiben (Abbildung 10). Es ist nicht ersichtlich und war womöglich auch den Betroffenen nicht klar, worauf diese Diskriminierung gegründet war.

Neben individuellen Einschränkungen des Postzugangs gab es kollektive Strafaktionen in der Form der Postsperre für eine Gruppe oder für das ganze Lager. So galt für Buchenwald in der Zeit vom 16. Januar bis 15. Februar 1941 
eine Postsperre, weil angeblich Kassiber ins Lager geschmuggelt worden waren. Auf Einlagezetteln (Abbildung 11), die vom Absender zu unterzeichnen und einem Brief beizufügen waren, wurde dies den Korrespondenzpartnern mitgeteilt.

\subsection{Juden}

Von Einschränkungen ihrer Überlebenschancen und damit auch der Teilnahme am Postverkehr waren Juden massiv betroffen. In allen Konzentrationslagern waren jüdische Häftlinge in noch höherem Maße als andere das Ziel von Willkür und Brutalität der SS. Sie wurden in abgetrennten Lagerabschnitten unter unsäglichen Bedingungen gefangen gehalten. Oft mussten sie wochenlang in überfüllten, schlecht gelüfteten Baracken auf Beton oder Erdboden schlafen, erhielten kaum Nahrung, hatten keine Gelegenheit zur Hygiene, durften sich bei Krankheit nicht ärztlich behandeln lassen, wurden in die 'Strafkompanien` gesteckt, in Steinbrüchen und andernorts durch Schwerstarbeit zugrunde gerichtet, in die Postenkette gejagt, »auf der Flucht« erschossen. ${ }^{44}$

Unter diesem Vorbehalt lässt sich sagen, dass der Postverkehr von Juden bis etwa 1939 keinen offiziellen Beschränkungen unterworfen war. Nach dem 9. November 1938 wurden die etwa 27000 jüdischen Männer, die in die Lager Dachau, Buchenwald und Sachsenhausen eingeliefert worden waren, soweit sie die Misshandlungen überlebt hatten, unter Druck gesetzt, mittels Korrespondenz ihre Auswanderung zu organisieren. Die Zeitspanne etwa zwischen Kriegsbeginn und März 1943 ist hinsichtlich des Postreglements für Juden eine Art Grauzone. Ob und gegebenenfalls in welchem Umfang Juden in dieser Zeit Zugang zum Postverkehr hatten, ist nicht bekannt. Immer wieder taucht in Berichten die Feststellung auf, dass Juden oft monatelang keine Post empfangen oder absenden durften. ${ }^{45}$ In Auschwitz durften Juden nur selten Post absenden oder empfangen. ${ }^{46}$

44 Vgl. zum Beispiel Emil Carlebach: Geschichte der Juden in Buchenwald, in: Hackett (Hrsg.): Der Buchenwald-Report, S. $195 \mathrm{ff}$.

45 So Kogon: Der SS-Staat, S. 129. In Mauthausen waren Häftlinge aus der UdSSR und »fast alle Juden« vom Postverkehr ausgeschlossen; vgl. Maršálek: Die Geschichte des Konzentrationslagers Mauthausen, S. 67, Anm. 1. In Neuengamme war jüdischen Gefangenen »der Empfang von Paketen [...] ebenso verboten wie - zumindest zeitweise - jeglicher Briefverkehr.«; vgl. Detlef Garbe: Neuengamme - Stammlager, in: Benz, Distel (Hrsg.): Der Ort des Terrors, Bd. 5, S. 315346, hier: S. 322.

46 Szymański: Briefe an die nächsten Angehörigen, S. 19; Iwaszko: Kontakt mit der Außenwelt, S. 517. 
Ab 1943 gab es dann eine Regelung, was immer ihr praktischer Wert gewesen sein mag, nach der Juden und "Angehörigen der Ostvölker« der Empfang und das Absenden von Briefen und Karten erlaubt sein sollte. Eine Verfügung des RSHA, gez. Müller, an das SS-Wirtschafts-Verwaltungshauptamt, Amtsgruppe D - Konzentrationslager - vom 30. März 1943 bestimmte: »Die Postempfangs- und Schreiberlaubnis sämtlicher jüdischer Schutzhäftlinge und Angehörigen der Ostvölker wird auf einmal innerhalb zweier Monate beschränkt. ${ }^{47}$

Die quantitative Reichweite dieser Verfügung kann für Juden nicht sehr groß gewesen sein. Denn ein halbes Jahr zuvor, am 2. Oktober 1942, hatte Himmler angeordnet, dass sämtliche im Reichsgebiet gelegenen Konzentrationslager >judenfrei` zu machen und sämtliche jüdischen Häftlinge in das Konzentrationslager Auschwitz und das >Kriegsgefangenenarbeitslager Lublin zu überstellen seien. Es erscheint unwahrscheinlich, dass die in Auschwitz und Lublin zusammengepferchten Juden generell die Gelegenheit zum Schreiben und Empfangen von Post hatten. Auf symbolhafte Weise verdeutlicht ein Aushang aus dem Konzentrationslager Auschwitz (Abbildung 12) den Ausschluss der Juden und Russen aus der Teilnahme am Postverkehr.

Es sind jedoch aus der Zeit nach dem 3. März 1943 einzelne Karten und Briefe überliefert, die offenbar von jüdischen Gefangenen stammen. So konnte ein jüdischer Häftling in Auschwitz, Hermann Rosenberg, am Postverkehr teilnehmen. Hierzu die Abbildung 13, ein Brief Rosenbergs, Poststempel Auschwitz, 31. Oktober 1944, an seine Ehefrau Grete Rosenberg in Wesermünde. Es liegt nahe, die Ursache der >Privilegierung ‘ des Briefschreibers in seinem Status nach NS-Recht zu sehen: Hermann Rosenberg war mit einer Nichtjüdin verheiratet. ${ }^{48}$

Bis Januar/Februar 1945 genossen in Deutschland jüdische Partner/Partnerinnen in Ehen mit einer nichtjüdischen Partnerin/einem nichtjüdischen Partner, also in so genannten Mischehen, gewisse >Privilegien`. Sie wurden in der Regel zwar diskriminiert und schikaniert, aber nicht deportiert. Ähnlich erging es den Kindern aus gemischt jüdisch-nichtjüdischen Ehen, so genannten Mischlingen. Es könnte sein, dass die Partner in >Mischehen $`$ und die >Mischlinge`, wenn sie denn, aus welchen Gründen auch immer, in ein Konzentrationslager gerieten, dort das >Privileg` der Teilnahme am Postverkehr erhielten. Dem Autor sind weitere Beispiele bekannt, die in diese Richtung weisen.

47 Reichssicherheitshauptamt: Schriftverkehr der Schutzhäftlinge, 30. März 1943. Quelle: BArch NS 3/426, Bl. 49.

48 Informationen zu Hermann Rosenberg nach Dokumenten, die in der KZ-Gedenkstätte Hailfingen/Tailfingen aufbewahrt werden. Ich danke Volker Mall, dem Leiter der Gedenkstätte, dass er mir diese Dokumente zugänglich gemacht hat. 
$\mathrm{Zu}$ Hermann Rosenberg liegen der KZ-Gedenkstätte Hailfingen einige inter-essante Unterlagen vor. Hiernach wurde er am 27. Juli 1943 ins Konzentrationslager Auschwitz eingeliefert. Zuvor hatte er im Polizeigefängnis Bremen in Erwartung eines Prozesses wegen angeblicher Devisenvergehen eingesessen. Aus Auschwitz wurde er nach Hailfingen, einem Außenlager des bereits befreiten KL Natzweiler, transportiert. Hier starb er am 25. Dezember 1944.

Es gab in einigen Konzentrationslagern genau abgegrenzte Gruppen jüdischer Häftlinge, die Post erhalten und absenden durften. $\mathrm{Zu}$ diesen Gruppen zählten die Angehörigen des Fälscherkommandos im KL Sachsenhausen. ${ }^{49} \mathrm{Zu}$ der Kategorie der >Privilegierten ‘ gehörten auch die slowakischen Jüdinnen im Stammlager Auschwitz, die in dem Häftlingskommando Politische Abteilung arbeiten mussten. ${ }^{50}$ Möglicherweise waren auch die tschechischen Juden, die Ende Juni 1944 aus dem Familienlager Birkenau in das Sachsenhausener Außenlager Schwarzheide transferiert wurden, hinsichtlich der Teilnahme am Postverkehr $>$ Privilegierte $\triangleleft{ }^{51}$

Der in etlichen Vernichtungsstätten und Konzentrationslagern von der SS inszenierte Versand von Postkarten durch Juden, zumeist solchen, die unmittelbar vor der Ermordung standen, ist nicht unter Korrespondenz zu subsumieren. Diese unter der Bezeichnung »Briefaktion des RSHA (Juden)« bekannt gewordene Operation hatte nicht den Zweck, eine Kommunikation zwischen Häftlingen und Personen außerhalb des Lagers herzustellen, sondern sie sollte Einzelpersonen und Judenräte beruhigen und helfen, noch nicht verhaftete Juden ausfindig zu machen. ${ }^{52}$ Zudem standen, außer im Fall des Familienlagers Birkenau, die meisten Kartenschreiber der >Briefaktion` bald nach dem Absenden der Karten nicht mehr zur Verfügung, da sie vergast waren. Die Juden im Familienlager hatten etwas mehr Zeit, wurden schließlich aber mit wenigen Ausnahmen ebenfalls ermordet.

\subsection{Roma und Sinti}

Roma und Sinti standen mit den Juden, den italienischen und den sowjetischen Gefangenen auf der untersten Stufe der Gefangenen-Hierarchie. Sie wurden mit ausgesuchter Brutalität behandelt und oft durch Misshandlungen, Nahrungsmittelentzug, Vorenthalten medizinischer Behandlung oder beim

49 Zum Fälscherkommando vgl. Wewer: Spuren des Terrors, S. 77f.

50 Lore Shelley (Hrsg.): Schreiberinnen des Todes, Bielefeld 1992 (zuerst Secretaries of Death, New York 1986).

51 Zum Außenlager Schwarzheide vgl. Wewer: Spuren des Terrors S. 85 f.

52 Zur »Briefaktion des RSHA (Juden)« vgl. Wewer: Postalische Zeugnisse zur deutschen Besatzungsherrschaft im Protektorat Böhmen und Mähren, S. $161 \mathrm{ff}$. 
>Fluchtversuch ermordet. Soweit bekannt, gibt es keine schriftlichen Regeln für den Postverkehr von Roma und Sinti.

Es sind jedoch einige postalische Dokumente aus Konzentrationslagern, die von Sinti stammen, bekannt. Eines der Dokumente, ein Faltbrief von Kinophas Schmidt aus dem Konzentrationslager Sachsenhausen, abgesandt am 23. Dezember 1944 an seine Mutter, ist als Abbildung 14 faksimiliert. Dass solche Zeugnisse sehr selten sind, ergibt sich schon daraus, dass dies die einzige Postsendung eines Sinto im Archiv des Dokumentations- und Kulturzentrums Deutscher Sinti und Roma in Heidelberg ist.

Eine in ihrer Erhaltung und ihrem Umfang erstaunliche Sammlung von Post, die Sinti aus Konzentrationslagern geschickt haben, befindet sich im Archiv der Erinnerungs- und Gedenkstätte Wewelsburg. Zwei dieser Dokumente sind im Begleitband zur ständigen Ausstellung der Gedenkstätte faksimiliert: ein Schreiben der Sintezza Adelgunde Rose aus dem Konzentrationslager Buchenwald an ihre Familie in Stettin vom 23. November $1944^{53}$ und ein Schreiben von Adelgunde Roses Bruder Alman Rose aus dem Konzentrationslager Niederhagen in Wewelsburg an die Eltern vom 7. November 1941..$^{54}$

Die Umstände, denen die genannten Dokumente ihr Überleben verdanken, sind nicht bekannt. Möglicherweise war es, wie so oft, eine Verkettung von Zufällen. Da aber offenbar die Mutter von Kinophas Schmidt im Dezember 1944 noch und da auch die Eltern der Geschwister Rose offenbar im November 1944 lebten und sich in Freiheit befanden, erscheint es möglich, dass beide Familien nach den rassistischen Maßstäben der SS als >privilegiert ' galten und sie vorläufig nicht deportiert wurden oder aber, im Falle der Deportation, das >Privileg`der Teilnahme am Postverkehr erhielten.

\subsection{Sowjetische Gefangene}

Bei den sowjetischen Gefangenen handelte es sich zum großen Teil um ehemalige Angehörige der Roten Armee, denen aufgrund irgendwelcher oder angeblicher Vergehen - etwa Fluchtversuch, Disziplinlosigkeit, Arbeitsverweigerung - der Status von Kriegsgefangenen entzogen worden war und die man in Konzentrationslager geschickt hatte. Von den seit Herbst 1941 in großer Zahl in Dachau, Sachsenhausen, Buchenwald, Mauthausen und Auschwitz eintreffenden sowjetischen Gefangenen wurden tausende ermordet. Juden und die ehemaligen Angehörigen der Roten Armee sollten prinzipiell die Lager nicht

53 Wulff E. Brebeck u. a. (Hrsg.): Endzeitkämpfer. Ideologie und Terror der SS, Berlin/München 2011, S. 235.

54 Ebenda, S. 309. 
lebend verlassen, ebenso wenig wie Sinti und Roma. Soweit bekannt, duften die ins $\mathrm{KZ}$ verschleppten ehemaligen Soldaten der Roten Armee überhaupt nicht am Postverkehr teilnehmen.

\subsection{Ostarbeiter}

Als Ostarbeiter galten Zivilarbeiter aus dem Gebiet der Sowjetunion. In der offiziellen Sprachregelung wurden sie auch als "Angehörige der Ostvölker» bezeichnet. Nach der bereits erwähnten Verfügung des RSHA vom 30. März 1943 und einer weiteren Verfügung des Wirtschaftsverwaltungshauptamtes vom 12. April $1943^{55}$ durften Ostarbeiter einmal in zwei Monaten Post empfangen und absenden. Hierbei galt die Bedingung, dass sie nur spezielle Postkarten mit Rückantwort benutzen durften. Für den Postverkehr der Ostarbeiter gab die Reichspost besondere Frage-und-Antwort-Ganzsachen mit deutscher, ukrainischer und russischer Inschrift heraus, die so genannten Ostarbeiter-Ganzsachen. ${ }^{56}$ Die Verfügung vom 12. April 1943 bestimmte, dass »bei den Briefsendungen der Ostarbeiter nicht zum Ausdruck kommen soll, daß diese sich in einem Konzentrationslager befinden. " Es seien daher "getarnte Absenderadressen« anzugeben.

Eine solche Sendung ist die Ganzsache Abbildung 15. Als Absende-Postamt ist Flossenbürg b. Weiden (Oberpfalz), Postamt II angegeben. Bei dem Absender Kazimierz Roksela handelte es sich vermutlich um einen Ostarbeiter, der in das Konzentrationslager Flossenbürg eingeliefert worden war.

Ostarbeiter wurden vorwiegend in der Rüstungsindustrie eingesetzt. In der nationalsozialistischen rassistischen Wertehierarchie standen sie auf dem vorletzten Platz oberhalb der Juden, Sinti und Roma. In Deutschland wurden sie mit äußerster Brutalität behandelt. Die Diskriminierung drückte sich unter anderem darin aus, dass sie auf dem Rücken einen Aufnäher OST tragen mussten. (Polen aus dem Generalgouvernement mussten ein P tragen.) Geringfügige Verstöße gegen eine der zahlreichen diskriminierenden Bestimmungen konnten Einweisung in ein Arbeitserziehungslager oder in ein Konzentrationslager zur Folge haben.

Weshalb die Tarnung des Absenders? Ostarbeiter galten in ihren Herkunftsländern offiziell als Freiwillige. In der Tat hatten viele sich aufgrund von Werbekampagnen der deutschen Arbeitsverwaltung gemeldet. Zumindest zu Anfang der deutschen Besatzung wussten viele nicht, was ihnen in Deutschland

55 NS-Wirtschafts-Verwaltungshauptamt: Amtsgruppenchef D, Schriftverkehr der Häftlinge,

12. April 1943. Quelle: BArch NS 3/426, Bl. 48.

56 Michel: Ganzsachen Deutschland 2014, Deutsches Reich P 310. 
blühte. Andere waren, oft von der Straße weg, zwangsrekrutiert worden. Ihnen allen wurde die Heimkehr verwehrt, so dass auch die Freiwilligen zu Zwangsarbeitern wurden. Die Daheimgebliebenen, darunter auch potenzielle Freiwillige, sollten nicht erfahren, dass sie als Arbeitskräfte in Deutschland ins Konzentrationslager geraten konnten.

\subsection{Strafkompanie und K-Kompanie}

Die in allen Lagern bestehenden Strafkompanien waren in dem barbarischen System die barbarischsten Einrichtungen. Die Zuordnung zur Strafkompanie war mit einem hohen Grad an Wahrscheinlichkeit ein Todesurteil. Juden und Zeugen Jehovas steckte die SS bevorzugt in Strafkompanien. Die Angehörigen der Strafkompanie durften nur alle Vierteljahre einen Brief oder eine Karte schreiben und empfangen. ${ }^{57}$ Die Vordruck-Postkarte aus Dachau (Abbildung 16), auf der es heißt, dass jeder Schutzhaftgefangene »alle 3 Monate einen Brief oder eine Karte von seinen Angehörigen empfangen und an sie absenden« dürfe, stammt vermutlich von einem Angehörigen der Strafkompanie.

Der Strafkompanie wurde nach Ausbruch des Krieges eine so genannte KKompanie angegliedert. »K-Häftlinge waren alle diejenigen, die im Kriege sich irgendwie vergangen hatten, aber nicht verurteilt werden konnten, da ihre >Straftat $<$ zu gering war. ${ }^{58}$ Die Bedingungen in der K-Kompanie waren die gleichen wie in der Strafkompanie, die Sterberate war infolgedessen gleich hoch. Max Pabst, der eine solche Kompanie überlebte, schreibt in seinen Erinnerungen: »Wir durften nur einmal vierteljährlich einen Brief an unsere Angehörigen schreiben und nur einen von ihnen empfangen. ${ }^{59}$

\subsection{Zeugen Jehovas}

Die Standhaftigkeit der Zeugen Jehovas in der Ablehnung des Regimes und des Kriegsdienstes erregte den besonderen Zorn der SS. Die Zeugen Jehovas wurden anfangs genau so brutal behandelt wie die Juden, später nutzte die SS ihre Friedfertigkeit und Zuverlässigkeit sowie ihre Ablehnung von Flucht, indem sie sie für Aufgaben heranzog, bei denen ein geringeres Maß an Bewachung möglich oder erforderlich war als üblich. Dennoch gehörten die Zeugen Jehovas - damals: `Ernste Bibelforscher` - zu den bevorzugten Aggressionsobjekten

57 Kogon: Der SS-Staat, S. 129.

58 Max Pabst: Die K-Kompanie, in: Hackett (Hrsg.): Der Buchenwald-Report, S. 270.

59 Ebenda, S. 271. 
der SS. Da sie sich weigerten, "von der Irrlehre der Bibelforscher abzulassen«, wie es auf dem großflächigen Stempel hieß, der auf die ihnen zur Verfügung stehenden Briefbögen gesetzt wurde und dort eine ganze Seite in Anspruch nahm, wurden sie durch eine Reduzierung des zugelassenen Briefverkehrs auf das Absenden und den Empfang je einer Postsendung pro Vierteljahr bestraft (Abbildung 17).

\subsection{Nacht-und-Nebel-Häftlinge}

Als Reaktion auf den Widerstand in den von Deutschland überfallenen westlichen Ländern erließ Hitler am 7. Dezember 1941 einen Geheimerlass unter der Bezeichnung »Richtlinien für die Verfolgung von Straftaten gegen das Reich oder die Besatzungsmacht in den besetzten Gebieten«. Vom Oberkommandierenden der Wehrmacht, Keitel, in Kraft gesetzt, sah der Erlass vor, dass Widerstandskämpfer oder des Widerstands Verdächtige in aller Heimlichkeit - bei Nacht und Nebel - in Haft zu nehmen waren. Sie sollten in völliger Isolation gehalten werden, ihre Angehörigen sollten keine Nachricht über ihren Verbleib erhalten, sie sollten >verschwinden gehörigen hiervon nicht benachrichtigt werden. Die NS-Führung versprach sich von diesem Vorgehen eine abschreckende Wirkung. Auf der Grundlage des Erlasses wurden an die 7000 Personen, davon etwa 5000 aus Frankreich, die übrigen aus den Niederlanden, Belgien, Norwegen und Luxemburg, nach Deutschland verschleppt und in Konzentrationslagern untergebracht. Das Isolationsgebot des Erlasses war für die Konzentrationslager unmittelbar geltendes Recht bzw. Unrecht. Über ihren Status wurden die Häftlinge im Unklaren gelassen.

Die NN-Häftlinge durften keine Post absenden oder erhalten. Es scheint hierzu eine Weisung des RSHA gegeben zu haben, die aber nicht erhalten ist. Denn in der bereits zitierten Verfügung des RSHA vom 30. März 1943 heißt es unter IV. a): »Der Post- und Paketempfang, sowie die Schreiberlaubnis für sämtliche NN-Häftlinge wird von der zu Ziffer I und II getroffenen Regelung nicht berührt.« Das ist eine etwas kryptische Formulierung, die genau das Gegenteil von dem meint, was sie suggeriert. Sie soll besagen, dass sich für die NN-Häftlinge an dem Verbot des Paketempfangs sowie des Absendens und Empfangs von Post nichts ändert. In einer Verfügung des WVHA vom 21. Juni 1943 findet sich ein Hinweis auf eine die NN-Häftlinge betreffende Regelung des WVHA vom 12. April 1943, die leider bisher auch nicht aufgetaucht ist. Es heißt hier: 
"Im übrigen weise ich auf das Schreiben DI/Az.: 14 d 4 /Ot./S.- vom 12.4.43 hin. Die darin getroffenen Regelungen bleiben auch weiterhin in Kraft, insbesondere soweit es sich um NN-Häftlinge handelt. ${ }^{60}$

Es sind zwei spezielle >Aktionen bekannt, die im Zuge des NN-Erlasses stattfanden. In der >Aktion Meerschaum` wurden zwischen Dezember 1942 und Spätsommer 1943 Franzosen verhaftet. >Aktion Frühlingswind ‘ wurde eine weitere Operation genannt, bei der Franzosen und Belgier verhaftet wurden. $\mathrm{Zu}$ den im Rahmen der >Aktion Meerschaum` Festgenommenen gibt es eine Anweisung des WVHA, Amtsgruppe D - Konzentrationslager - vom 6. Juli 1943, Unterschrift Liebehenschel, an die Kommandanten der Konzentrationslager Sachsenhausen, Buchenwald, Mauthausen und Ravensbrück. Sie lautet:

»Der Chef der Sicherheitspolizei und des SD hat im Einvernehmen mit dem BdS, Paris entschieden, dass die Häftlinge aus der Aktion Meerschaum hinsichtlich des Briefverkehrs den allgemeinen für KL.-Häftlinge geltenden Bestimmungen unterliegen.

Es besteht danach nur die einzige Ausnahme, dass die Mitteilung vom Ableben eines Häftlings aus der Aktion Meerschaum auf keinen Fall an die Angehörigen, sondern an den BdS, Paris zu richten ist. ${ }^{61}$

Offenbar hatte die Gestapo Paris durch das Verschwindenlassen von Widerstandskämpfern den Widerstand in Frankreich erst recht angefacht und wollte nun durch die Genehmigung von Postverkehr die Wogen etwas glätten. Über den etwaigen Tod eines Häftlings sollten die Angehörigen aber im Unklaren und also in ständiger Sorge gehalten werden.

Im Frühsommer 1943 ordnete das RSHA die Überstellung der >germanischen NN-Häftlinge, zu denen offenbar auch die Franzosen gehörten, in das

60 NS-Wirtschafts-Verwaltungshauptamt: Amtsgruppenchef D, Schriftverkehr fremdländischer Häftlinge und von NN-Häftlingen, 21. Juni 1943. Quelle: BArch NS 3/426, Bl. 88.

61 NS-Wirtschafts-Verwaltungshauptamt: Amtsgruppenchef D, Aktion Meerschaum, 6. Juli 1943. Quelle: BArch NS 3/426, Bl. 98. Die Abkürzung BdS steht für Befehlshaber der Sicherheitspolizei und des SD [Sicherheitsdienstes]. Die BdS waren Außenstellen des Reichssicherheitshauptamtes (RSHA) in den besetzten Gebieten. Sie hatten ähnliche Funktionen wie die Einsatzgruppen. Ebenso wie zwischen RSHA und Einsatzgruppen gab es eine starke personelle Fluktuation zwischen BdS und Einsatzgruppen. Der BdS Frankreich mit Sitz in Paris war von Mai 1940 bis September 1944 SS-Standartenführer Dr. Helmut Knochen. Er war ab Mai 1942 der Hauptorganisator der Deportation der französischen Juden. 1954 in Paris zum Tode verurteilt, 1962 entlassen. In der Bundesrepublik in der Hilfsorganisation für NS-Täter Stille Hilfe tätig. Ernst Klee: Personenlexikon zum Dritten Reich, Frankfurt a.M. 2011. S. 320. Sein Nachfolger als BdS Frankreich war ab November 1944 SS-Obersturmbannführer Friedrich Suhr. November 1942 bis August 1943 war er Führer des Sonderkommandos 4b gewesen, danach Führer des Einsatzkommandos 6. 1946 Suizid in Haft in Wuppertal-Elberfeld. Ebenda, S. 616. 
Konzentrationslager Natzweiler an. ${ }^{62}$ Hier wurden sie einer äußerst brutalen Behandlung unterworfen, die für viele zum Tode führte.

\subsection{Republikanische Spanier}

In einer Verfügung der Abteilung III/Poststelle des Konzentrationslager Dachau vom 17. Mai 1943 findet sich eine Bestimmung über die Teilnahme von »ehem. Rotspanienkämpfern spanischer Nationalität« am Postverkehr. ${ }^{63}$ Bei dieser Gruppe von Häftlingen, die zumeist als republikanische Spanier bezeichnet werden, handelte es sich um ehemalige Soldaten der von den Truppen Francos geschlagenen republikanischen Armee. (Sie dürfen nicht verwechselt werden mit den Interbrigadisten, den aus zahlreichen Ländern stammenden Freiwilligen, die in den Internationalen Brigaden an der Seite der Republik im Spanischen Bürgerkrieg gekämpft haben.) Etwa 15000 republikanische Spanier, die sich nach Frankreich geflüchtet hatten und die zuerst von der demokratischen Regierung, dann vom Vichy-Regime interniert worden waren, gerieten bei der Besetzung der bisher unbesetzten Zone Frankreichs durch die Deutschen in deren Gewalt. Die Gestapo nahm einige tausend von ihnen gefangen und verschleppte sie in deutsche Konzentrationslager. Allein nach Mauthausen wurden 7000 republikanische Spanier deportiert. Etwa 5000 von ihnen wurden ermordet, vorwiegend in den Steinbrüchen. ${ }^{64}$ Es wird berichtet, dass diese Spanier sich wegen ihrer tapferen und solidarischen Haltung großer Achtung bei den Mitgefangenen erfreuten.

Für die Überlebenden dieser stark dezimierten Häftlingsgruppe gab es ein merkwürdig anmutendes Postreglement. Sie durften monatlich zwei Briefe empfangen, aber nur alle sechs Wochen einen Brief absenden. In den Absendern auf den Briefen nach Spanien durften die Gefangenennummer, die BlockNummer und die Bezeichnung >Konzentrationslager` nicht erscheinen. Die Häftlinge mussten neutrale Deckadressen mit der Ortsbezeichnung »Dachau 3/K, bei München« verwenden.

62 Karin Orth: Das System der nationalsozialistischen Konzentrationslager, Zürich/München 2002, S. $98 \mathrm{f}$.

63 Konzentrationslager Dachau, Abteilung III/Poststelle: Allgemeine Anordnungen für den Briefverkehr und Paketempfang der Häftlinge im Konzentrationslager Dachau, 17. Mai 1943. Quelle: KZ-Gedenkstätte Dachau, Dokument 2109.

64 Maršálek: Die Geschichte des Konzentrationslagers Mauthausen, S. 363 ff. 
Die Welt und auch der Hitler-Freund Franco sollten also nicht erfahren, dass die ehemaligen spanischen Soldaten völkerrechtswidrig in Konzentrationslagern gefangen gehalten wurden. ${ }^{65}$

\subsection{Italiener}

Nach dem Frontwechsel Italiens im September 1943 nahmen die Deutschen etwa 600000 italienische Soldaten gefangen. Knapp 200000 von ihnen optierten für Mussolini und wurden entlassen. Gut 400000 Soldaten wurden als »Italienische Militärinternierte« zur Zwangsarbeit oder ins Konzentrationslager verschleppt.

»Zur Strafe« für den »Verrat« wurden die in Konzentrationslager Deportierten von der Teilnahme am Postverkehr ausgeschlossen. Diese Bestimmung, berichtet Gabriele Bergner aufgrund des Studiums von Erinnerungsberichten, wurde von den italienischen Gefangenen als große Belastung empfunden. ${ }^{66}$ Ihre Archivrecherchen in den Berichten ehemaliger Häftlinge bestätigen auch, dass die italienischen Häftlinge, von ganz wenigen Ausnahmen abgesehen, keine Pakete erhielten, was zu Spannungen mit solchen Gefangenen führte, die in den Genuss von Paketsendungen kamen. ${ }^{67}$ Vermutlich sollte durch die totale Postsperre, ähnlich wie im Falle der republikanischen Spanier, nur mit noch drastischeren Mitteln, die völkerrechtswidrige Konzentrationslager-Haft der italienischen Soldaten vor den Resten des Mussolini-Regimes und der internationalen Öffentlichkeit verborgen werden.

In den Konzentrationslagern und zum Teil auch in der Privatwirtschaft wurden die »Italienischen Militärinternierten« sehr schlecht behandelt und hatten eine große Zahl von Todesfällen zu beklagen. Mit fadenscheinigen Argumenten hat sich die Bundesrepublik Deutschland bis heute geweigert, den Überlebenden für die in deutschen Lagern und in deutscher Zwangsarbeit erlittenen physischen und seelischen Qualen eine Entschädigung zu zahlen. Alle italienischen Regierungen haben den Skandal hingenommen, um es nicht mit dem großen Nachbarn zu verderben.

65 Eines der wenigen überlieferten Exemplare dieser Kategorie von Häftlingspost ist reproduziert in: Julien Lajournade: Le Courrier dans les Camps de Concentration 1933-1945. Système et Rðle Politique, Paris 1989, S. 66.

66 Bergner: Aus dem Bündnis hinter den Stacheldraht, S. 197.

67 Ebenda, S. 199. 


\subsection{Luxemburger Polizeibeamte}

Im Konzentrationslager Dachau waren 17 ehemalige Polizeibeamte aus Luxemburg inhaftiert, die es abgelehnt hatten, im besetzten Jugoslawien Dienst für die Deutschen zu tun. ${ }^{68}$ Und in Buchenwald befanden sich 26 Angehörige einer Luxemburger Polizei-Freiwilligen-Kompanie, die sich im August 1941 geweigert hatten, sich von den Deutschen für die Partisanenbekämpfung ausbilden zu lassen. ${ }^{69}$ Für diese und andere Luxemburger Polizeibeamte galten spezielle Bedingungen. In der RSHA-Verfügung vom 30. März 1942 heißt es:

»IV. b) Bei den ehem. luxemburgischen Polizeibeamten verbleibt es bei den [sic] ergangenen RFSS-Befehl, nach welchem diese nur 1 mal im Jahr Post empfangen und ebenfalls nur 1 mal im Jahr schreiben dürfen, damit sie keinen ungünstigen Beeinflussungen aus der Heimat ausgesetzt sind.«

\subsection{Häftlinge im Konzentrationslager Lublin-Majdanek}

Nach Janusz Mozdzan wurde der großen Mehrheit der Häftlinge im Konzentrationslager Lublin-Majdanek erst im September 1943 ein geregelter Postverkehr erlaubt. ${ }^{70}$ Allerdings galten mindestens für die Zeit vom 4. März bis 21 . Juli 1944 Sonderbestimmungen. Nach einem auf einen Brief aufgeklebten Zettel waren erlaubt:

$\begin{array}{ll}» \text { Pakete bis zu } 2 \mathrm{~kg} . & -1 \text { mal jede Woche } \\ \text { Briefe } & -1 \text { mal im Monat } \\ \text { Karten } & -1 \text { mal jede } 14 \text { Tage. }{ }^{71}\end{array}$

Mit dem Erlass Himmlers vom 29. Oktober 1942 war den Häftlingen der Empfang von Paketen ohne Begrenzung der Zahl und des Gewichts gestattet worden. In Bezug auf Paketempfang war die Sonderregelung des Lagers LublinMajdanek also eine gravierende Einschränkung. Auch in Bezug auf Briefverkehr war sie eine Einschränkung. Denn statt zwei Briefe oder Karten pro Monat in Abgang und Eingang sah sie einen Brief und zwei Karten vor, im Saldo also eine Postsendung weniger als allgemein erlaubt.

68 Stanislav Zámečnik: Das war Dachau, Frankfurt a.M. 2010, S. 355.

69 Harry Stein (Red.): Konzentrationslager Buchenwald 1937-1945. Begleitband zur ständigen historischen Ausstellung, Göttingen 2011 (zuerst 1999), S. 172.

70 Janusz Mozdzan: Postgeschichte des Konzentrationslagers Lublin-Majdanek. Über das Lager, Briefe und Menschen, Manching 2010, S. 122.

71 Ebenda, S. 144. 
Nach dem "Auszug aus der Lagerordnung « auf einer Vordruck-Postkarte mit Poststempel 17. März 44 (Abbildung 18) galt zu diesem Zeitpunkt sogar eine noch restriktivere Regelung: Pro Monat durfte nur ein Brief oder eine Karte empfangen und abgesandt werden. Wie so oft, scheinen gleichzeitig unterschiedliche Regelungen gegolten zu haben. Das wirft ein Licht auf den Wert der Regelungen zum Postverkehr. Sie konnten in der Praxis jederzeit verändert oder ignoriert werden.

Die Karte aus Lublin-Majdanek weist zwei weitere Besonderheiten auf: Sie ist auf Polnisch geschrieben (sonst war es eiserne Regel, dass nur Deutsch zugelassen war), und sie ist über das Polnische Rote Kreuz gelaufen. Der Absender der Postkarte war Antoni Ostaszewski, ein in Polen hochangesehener Militärarzt. Ostaszewski wurde 1942 von der Gestapo verhaftet und nach Majdanek deportiert. Von hier schickten ihn die Deutschen zuerst nach GroßRosen und dann nach Sachsenhausen. Er überlebte und starb 1946.

\subsection{3 >Transport-Juden}

In einer Verfügung der Abteilung III/Poststelle des Konzentrationslagers Dachau vom 12. September 1944 heißt es: »Der Schriftverkehr von TransportJuden hat in jedem Falle zu unterbleiben. ${ }^{72}$ Als >Transport-Juden wurden solche als ‘arbeitsfähig`geltenden jüdischen Häftlinge bezeichnet, die ab Juni 1944 auf Anordnung des RSHA aus Auschwitz oder direkt aus Ungarn in westlich gelegene Lager transportiert wurden, um dort und in den Außenlagern in Rüstungsbetrieben $\mathrm{zu}$ arbeiten. ${ }^{73}$ Hierzu passt, dass die genannte Verfügung der Poststelle Dachau an »a 11 e SS-Arbeitslager und Aussenkommandos« gerichtet ist. Nicht erklärbar ist, wieso der Begriff >Transport-Juden ‘ bereits in dem bereits zitierten Dokument der Dachauer Poststelle vom 17. Mai 1943 auftaucht.

Wie sich aus Referenzangaben in den beiden genannten Dokumenten ergibt, existierten weitere Verfügungen oder Erlasse zum Status der >Transport-Juden «. Diese sind nach Kenntnis des Autors nicht überliefert.

Was immer in Vermerken und Verfügungen zum Postverkehr von Juden gestanden haben mag - für die meisten jüdischen Häftlinge war es bedeutungslos. Am 12. September 1944, dem Zeitpunkt der zuerst genannten Verfügung der Dachauer Poststelle, hatte kaum einer der herantransportierten Juden noch Verwandte oder Freunde, mit denen er hätte korrespondieren können. Wer

72 Konzentrationslager Dachau, Abteilung III/Poststelle: Schriftverkehr jüdischer Häftlinge,

12. September 1944. Quelle: KZ-Gedenkstätte Dachau, Archivkopie 3484/2.

73 Vgl. Maršálek: Die Geschichte des Konzentrationslagers Mauthausen, S. 429. 
nicht ermordet oder elend zugrunde gegangen war, befand sich irgendwo »auf Transport«. Alle waren, wie es auf dem bekannten Klebezettel der Deutschen Reichspost heißt, »abgereist ohne Angabe der Adresse«.

\section{Literatur}

Gabriele Bergner: Aus dem Bündnis hinter den Stacheldraht. Italienische Häftlinge im KZ Dachau 1943-1945. Deportationen und Lebensbedingungen, Hamburg 2002.

Wulff E. Brebeck u. a. (Hrsg.): Endzeitkämpfer. Ideologie und Terror der SS, Berlin/ München 2011.

Barbara Distel: Lebensbedingungen im Stammlager (Arbeit und Vernichtung), in: Wolfgang Benz, Barbara Distel (Hrsg.): Der Ort des Terrors, Bd. 5: Hinzert, Auschwitz, Neuengamme, München 2007, S. 99-106.

Klaus Drobisch, Günther Wieland: System der NS-Konzentrationslager 1933-1939, Berlin 1993.

Stefanie Endlich: Lichtenburg, in: Wolfgang Benz, Barbara Distel (Hrsg.): Der Ort des Terrors, Bd. 2: Frühe Lager, Dachau, Emslandlager, München 2005, S. 151-159.

Detlef Garbe: Neuengamme - Stammlager, in: Wolfgang Benz, Barbara Distel (Hrsg.): Der Ort des Terrors, Bd. 5, S. 315-346.

David A. Hackett (Hrsg.): Der Buchenwald-Report, München 1996.

Heinrich Heeren: Der Postverkehr mit den Emslandlagern 1870 bis 1950, Papenburg 2006.

Tadeusz Iwasko: Kontakt mit der Außenwelt, in: Wacław Długoborski, Francisek Piper (Hrsg.): Auschwitz 1940-1945, Bd. 2, Oświęcim 1999.

Ernst Klee: Personenlexikon zum Dritten Reich, Frankfurt a.M. 2011.

Habbo Knoch: Die Emslandlager 1933-1945, in: Wolfgang Benz, Barbara Distel (Hrsg.): Der Ort des Terrors, Bd. 2, S. 533-570.

Eugen Kogon: Der SS-Staat, Frankfurt a. M. o. J.

Erich Kosthorst, Bernd Walter (Hrsg.): Konzentrations- und Strafgefangenenlager im Dritten Reich. Beispiel Emsland, Band 1, Düsseldorf 1983.

Erich Kosthorst, Bernd Walter: Konzentrations- und Strafgefangenenlager im Emsland 1933-1945. Zum Verhältnis von NS-Regime und Justiz, Düsseldorf 1985.

Julien Lajournade: Le Courrier dans les Camps de Concentration 1933-1945. Système et Rðle Politique, Paris 1989.

Erik Lørdahl: German Concentration Camps 1933-1945. History and Inmate Mail, 2 Bde., davon Bd. II in zwei Teilen, Tårnåsen (Norwegen) 2000.

Hans Maršálek: Die Geschichte des Konzentrationslagers Mauthausen, Wien 2006.

Günter Morsch, Susanne zur Nieden (Hrsg.): Jüdische Häftlinge im Konzentrationslager Sachsenhausen 1936-1945, Berlin 2004.

Janusz Mozdzan: Postgeschichte des Konzentrationslagers Lublin-Majdanek. Über das Lager, Briefe und Menschen, Manching 2010.

Karin Orth: Das System der nationalsozialistischen Konzentrationslager, Zürich/München 2002.

Max Pabst: Die K-Kompanie, in: David A. Hackett (Hrsg.): Der Buchenwald-Report, S. 270.

Walter Poller: Arztschreiber in Buchenwald, Offenbach a. M. 1960. 
Hans-Günter Richardi: Schule der Gewalt. Die Anfänge des Konzentrationslagers Dachau, München 1982.

Arnold Weiss-Rüthel: Nacht und Nebel. Ein Sachsenhausen-Buch, Berlin/Potsdam 1949.

Jules Schelvis: Vernichtungslager Sobibór, Hamburg/Münster 2013.

Lore Shelley (Hrsg.): Schreiberinnen des Todes, Bielefeld 1992.

Jörg Skriebeleit: Flossenbürg - Stammlager, in: Wolfgang Benz, Barbara Distel (Hrsg.): Der Ort des Terrors, Bd. 4: Flossenbürg, Mauthausen, Ravensbrück, München 2007, S. 17-66.

Harry Stein (Red.): Konzentrationslager Buchenwald 1937-1945. Begleitband zur ständigen historischen Ausstellung, Göttingen 2011.

Elke Suhr: Die Emslandlager. Die politische und wirtschaftliche Bedeutung der emsländischen Konzentrations- und Straflager 1933-1945, Bremen 1985.

Tadeusz Szymański: Briefe an die nächsten Angehörigen - Lebenszeichen aus Auschwitz, in: Jadwiga Mateja (Red.): Znaki Pamięci. Korespondencja obozowa więźniów KL Auschwitz - Briefmarken Zeichen des Gedenkens. Häftlingsbriefe aus dem KL Auschwitz, Oświęcim 1995.

Johannes Tuchel: Nationalsozialistische Herrschaftssicherung und Verfolgungspraxis 1933 bis 1937, in: Volkhard Knigge u. a. (Hrsg.): Buchenwald Ausgrenzung und Gewalt 1937 bis 1945, Ausstellungsbegleitbuch, Göttingen 2016, S. 236-243.

Johannes Tuchel: Die Wachmannschaften der Konzentrationslager 1939 bis 1945 - Ergebnisse und offene Fragen der Forschung, in: Alfred Gottwaldt, Norbert Kampe, Peter Klein (Hrsg.): NS-Gewaltherrschaft. Beiträge zur historischen Forschung und juristischen Aufarbeitung, Berlin 2005, S.135-151.

Johannes Tuchel: Die Inspektion der Konzentrationslager 1938-1945. Das System des Terrors, Berlin 1994.

Nikolaus Wachsmann: kl, Die Geschichte der nationalsozialistischen Konzentrationslager, München 2016.

Heinz Wewer: Postalische Zeugnisse zur deutschen Besatzungsherrschaft im Protektorat Böhmen und Mähren, 2., durchges. Aufl., Berlin 2018.

Heinz Wewer: Spuren des Terrors. Postalische Zeugnisse zum System der deutschen Konzentrationslager, Berlin/Leipzig 2020.

Stanislav Zámečnik: Das war Dachau, Frankfurt a.M. 2010. 


\section{Abbildungen}

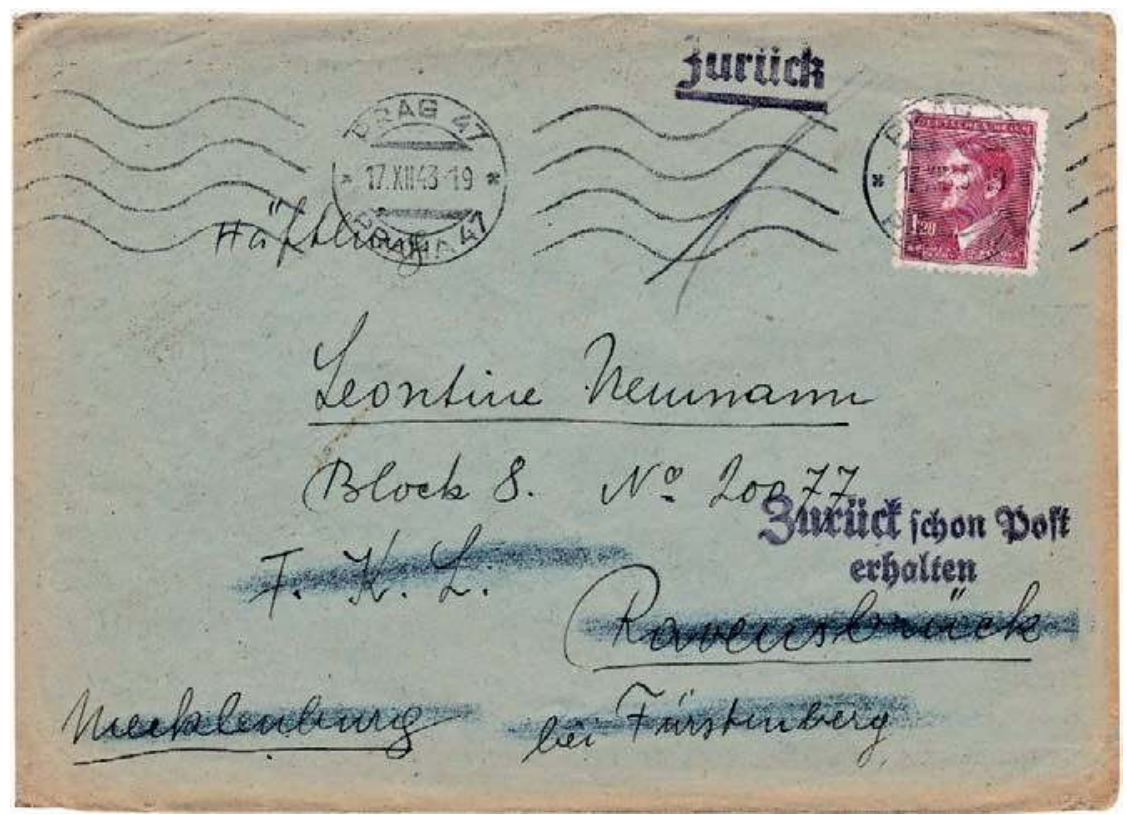

Abbildung 1: "Zurück schon Post erhalten«. Rücksende-Vermerk auf einem Brief an Leontine Neumann im Konzentrationslager Ravensbrück. 


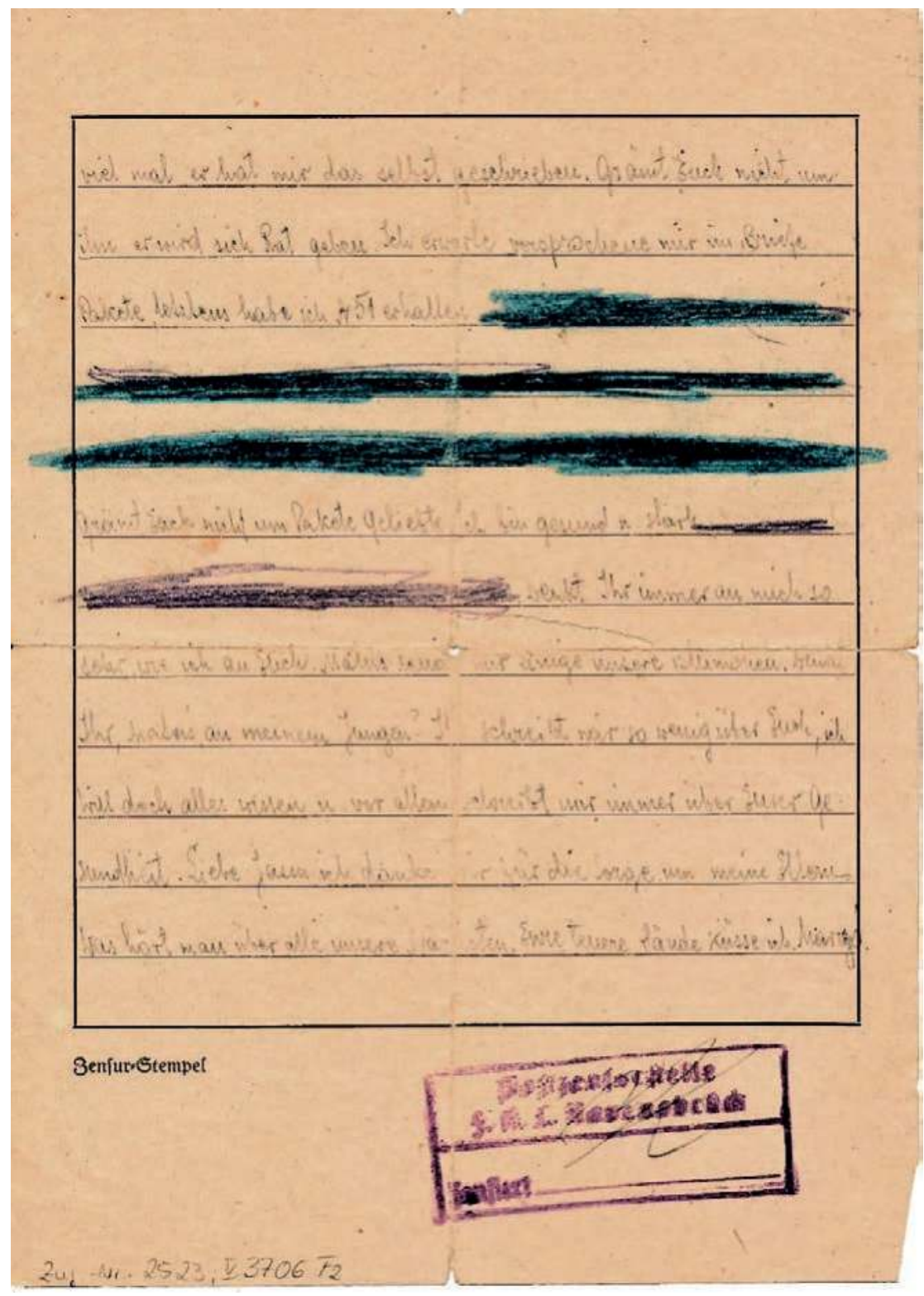

Abbildung 2: Durch Farbe unlesbar gemachte Passagen auf dem Brief eines Häftlings aus dem Konzentrationslager Ravensbrück. 


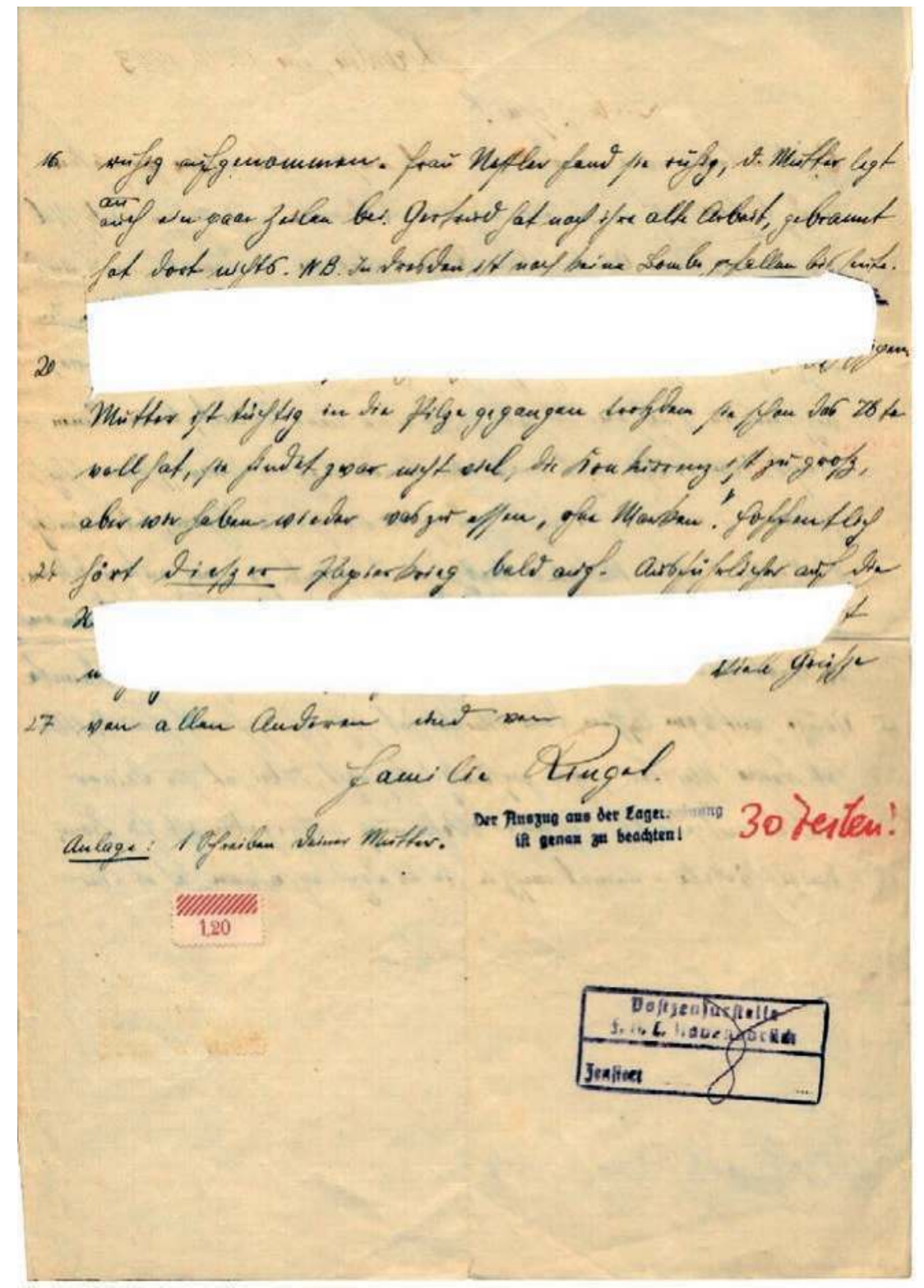

Abbildung 3: Durch Ausschnitte unlesbar gemachte Passagen eines Briefes an einen Häftling im Konzentrationslager Ravensbrück. 


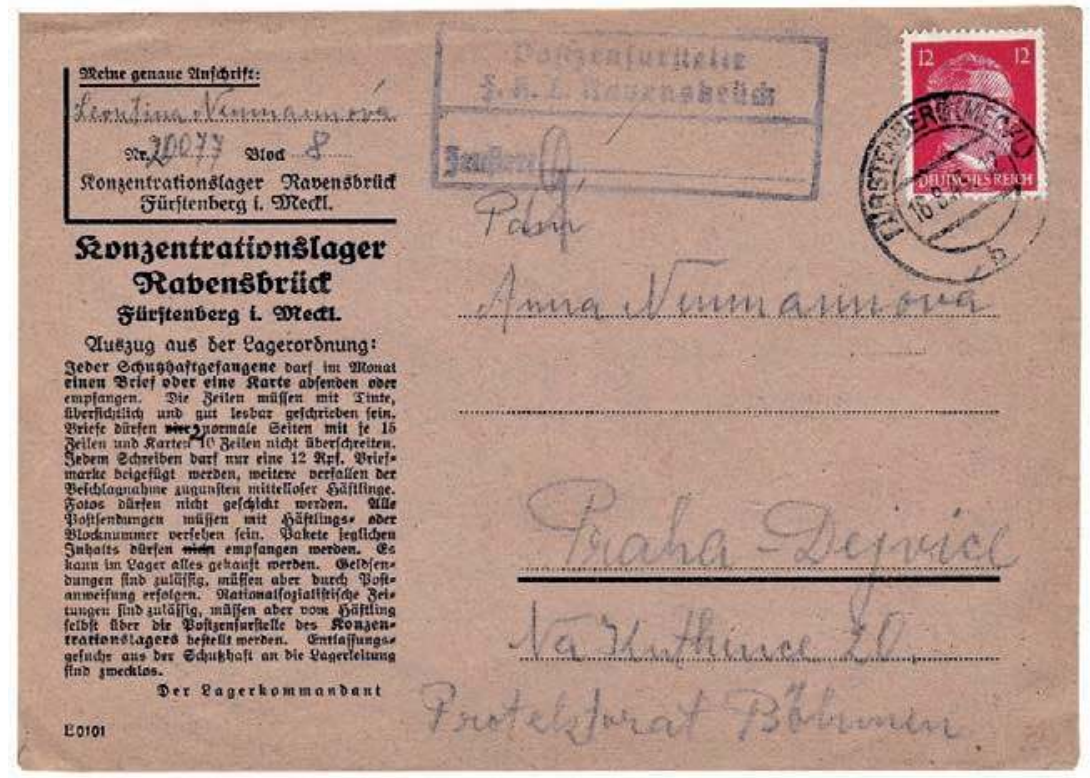

Abbildung 4: Pakete jeglichen Inhalts dürfen empfangen werden. Korrigierter VordruckBriefumschlag des Häftlings Leontine Neumann aus dem Konzentrationslager Ravensbrück.

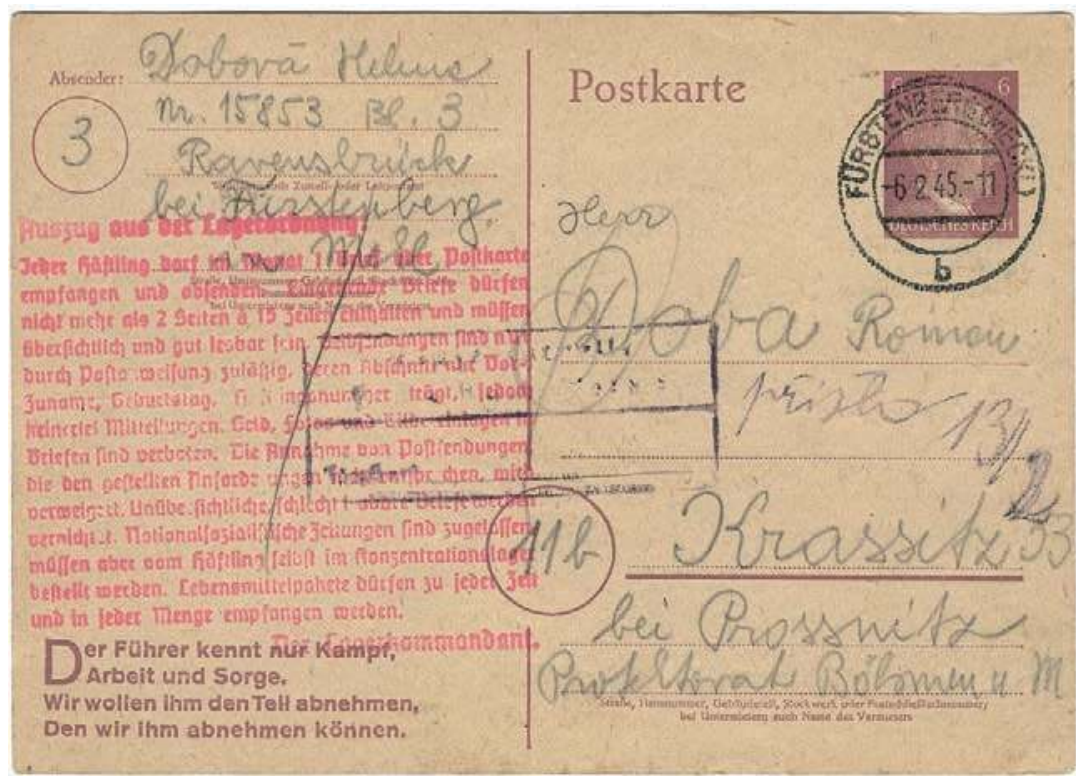

Abbildung 5: »Lebensmittelpakete dürfen zu jeder Zeit und in jeder Menge empfangen werden.» Ganzsache des Häftlings Helena Dobová aus dem Konzentrationslager Ravensbrück. 


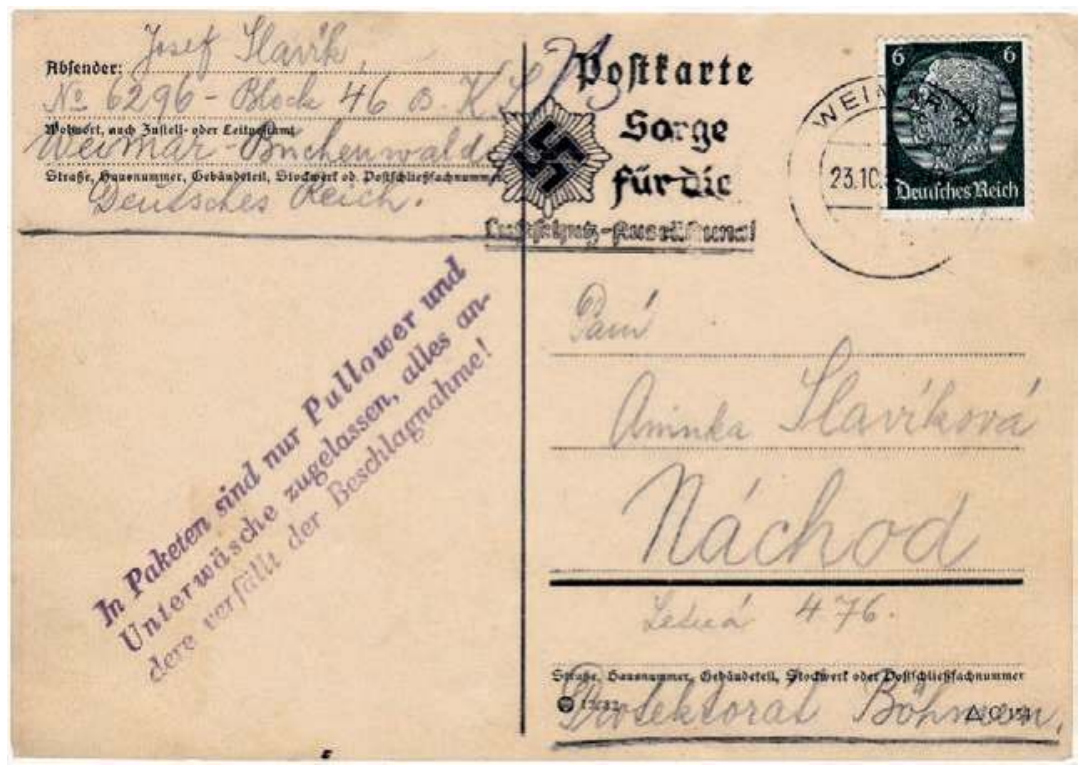

Abbildung 6: „In Paketen sind nur Pullower und Unterwäsche zugelassen, alles andere verfällt der Beschlagnahme!« Postkarte des Häftlings Josef Slavik aus dem Konzentrationslager Buchenwald.

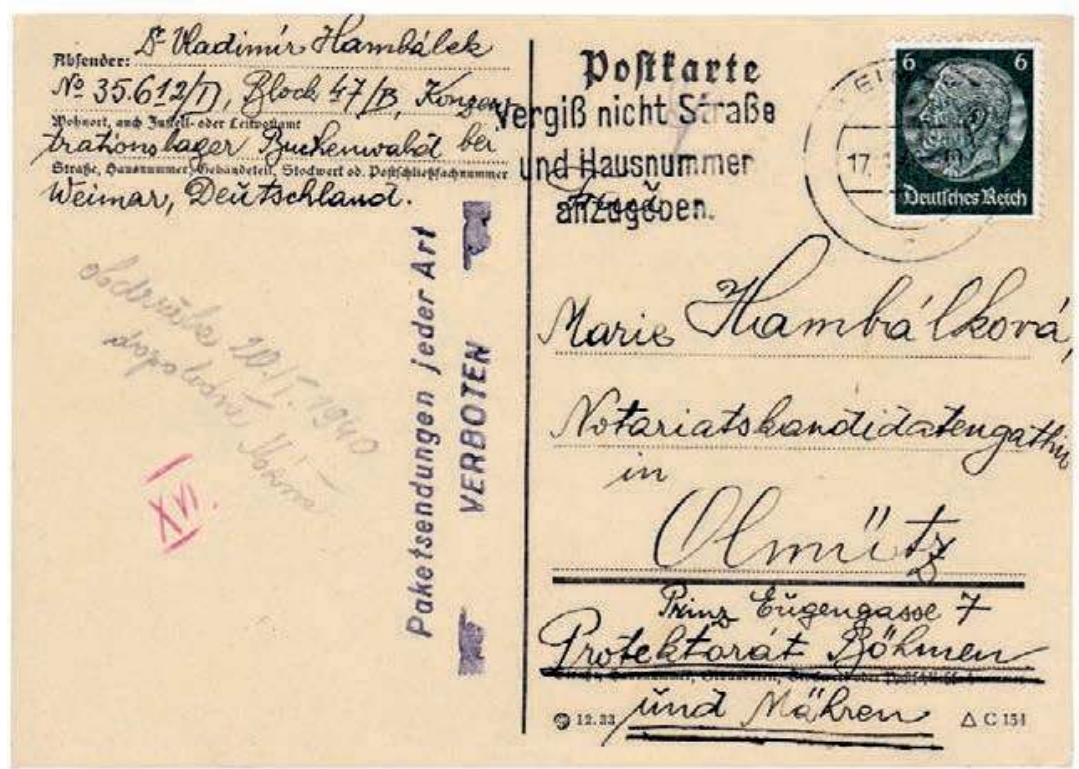

Abbildung 7: „Paketsendungen jeder Art/VERBOTEN«. Postkarte des Häftlings Vladimír Hambálek aus dem Konzentrationslager Buchenwald. 


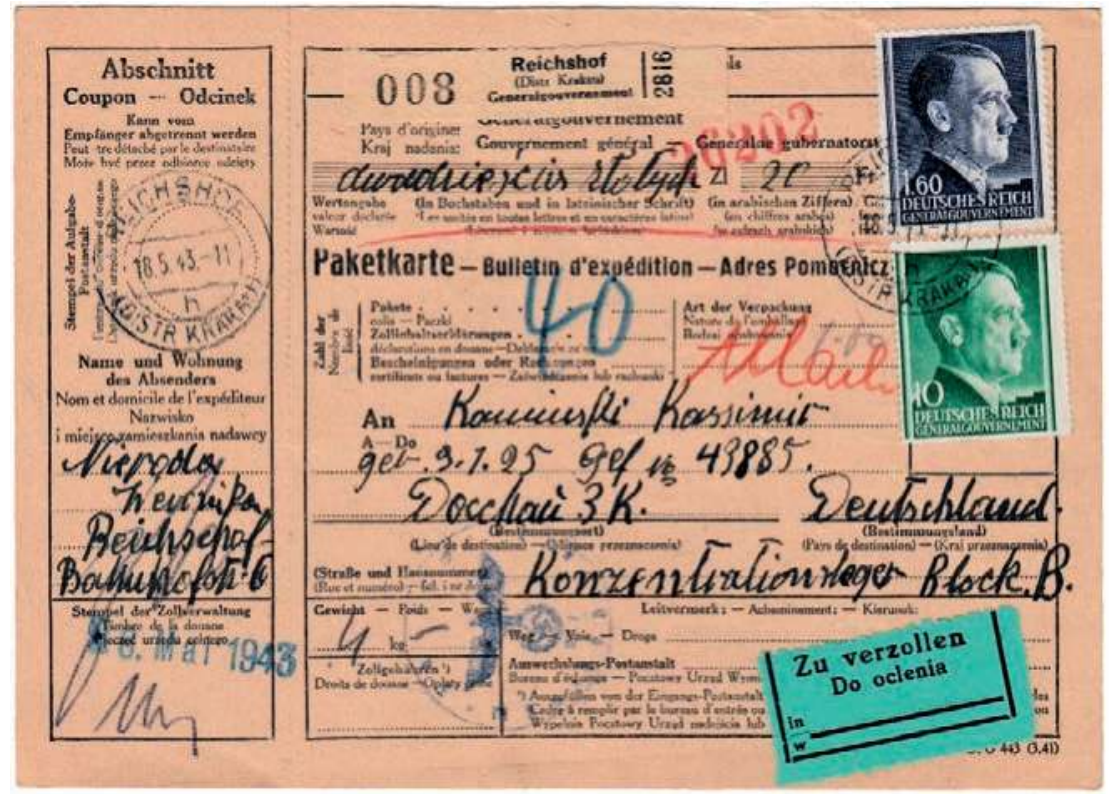

Abbildung 8: Paketkarte für ein Wertpaket an Kassimir Kaminski, Häftling im Konzentrationslager Dachau.

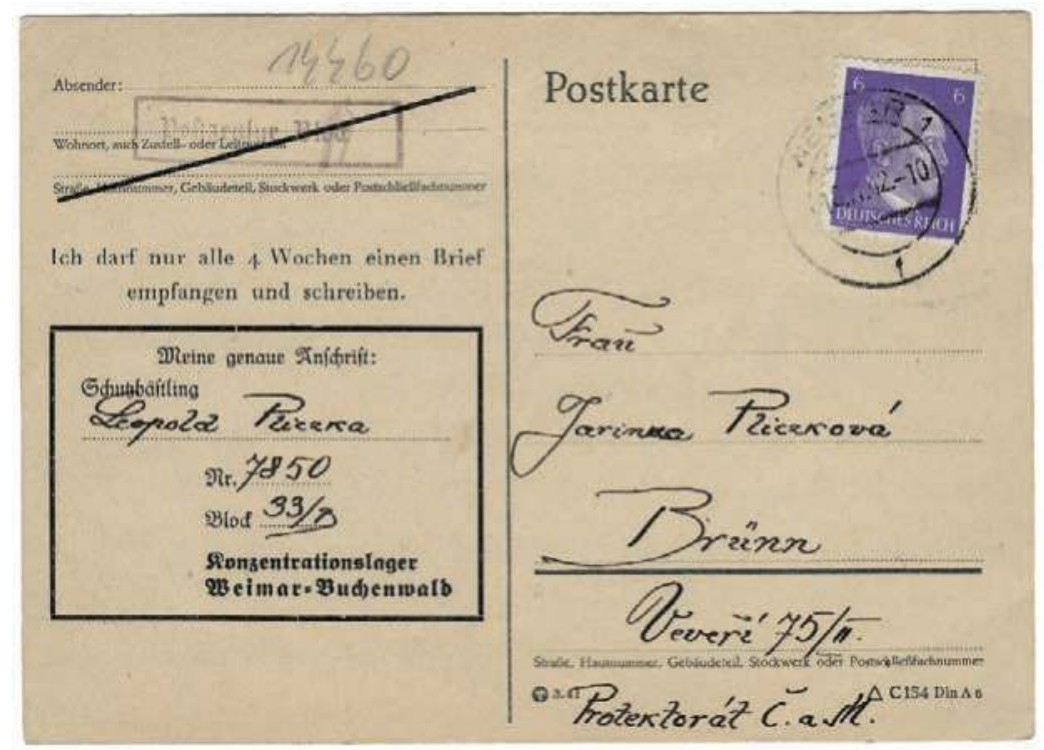

Abbildung 9: "Ich darf nur alle 4 Wochen einen Brief empfangen und schreiben«. Postkarte des Häftlings Leopold Pliczka aus dem Konzentrationslager Buchenwald. 


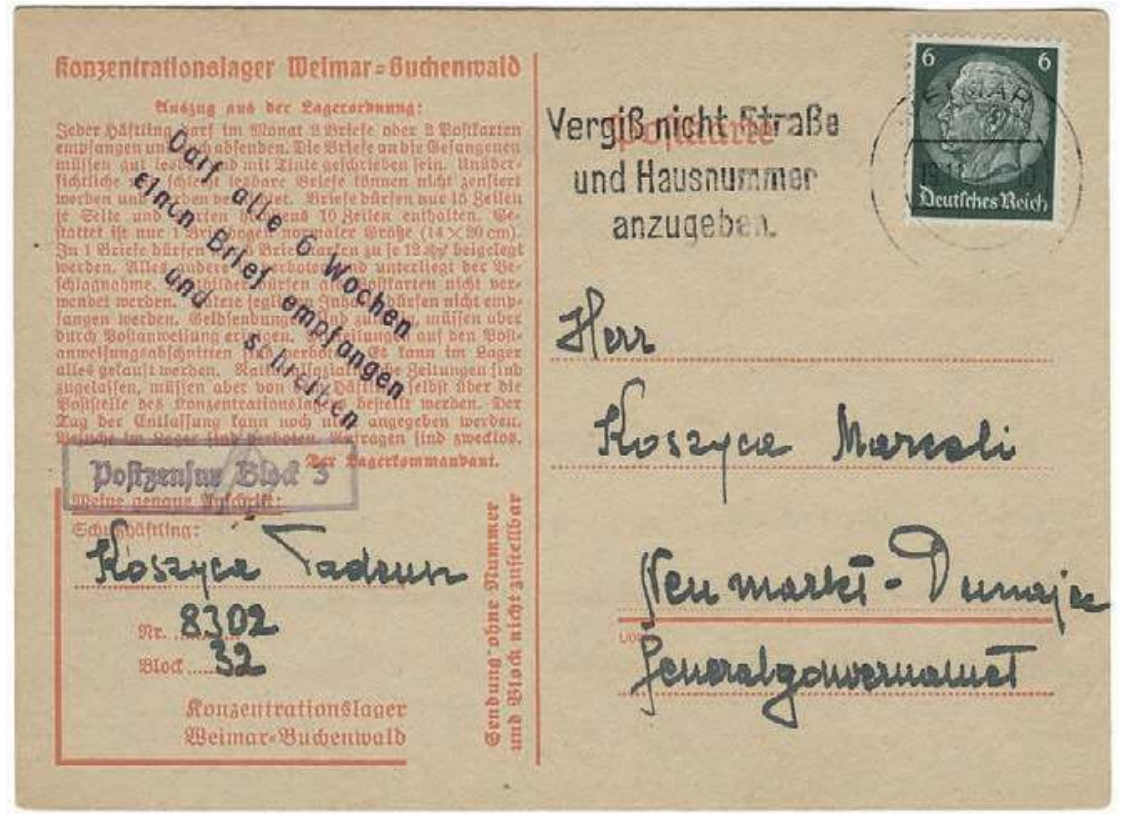

Abbildung 10: »Darf alle 6 Wochen/einen Brief empfangen/und schreiben«. Nebenstempel auf Vordruckpostkarte des Häftlings Tadeusz Koszyca aus dem Konzentrationslager Buchenwald.

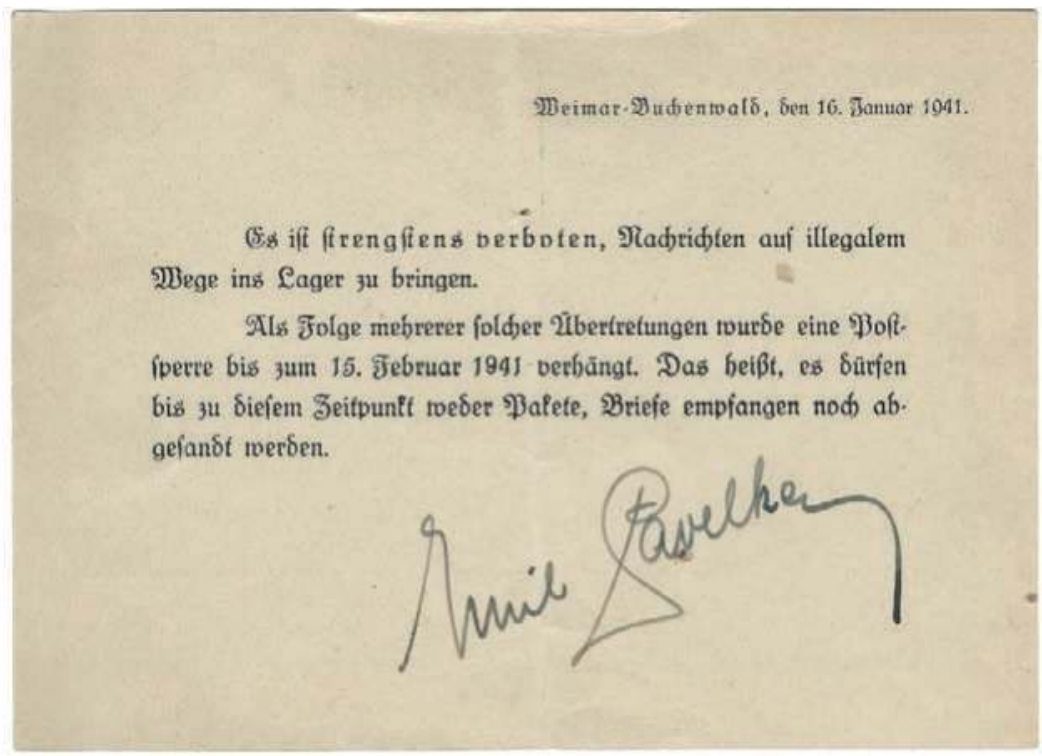

Abbildung 11: Postsperre im Konzentrationslager Buchenwald. Einlegezettel mit Unterschrift des Häftlings Emil Pawelka. 


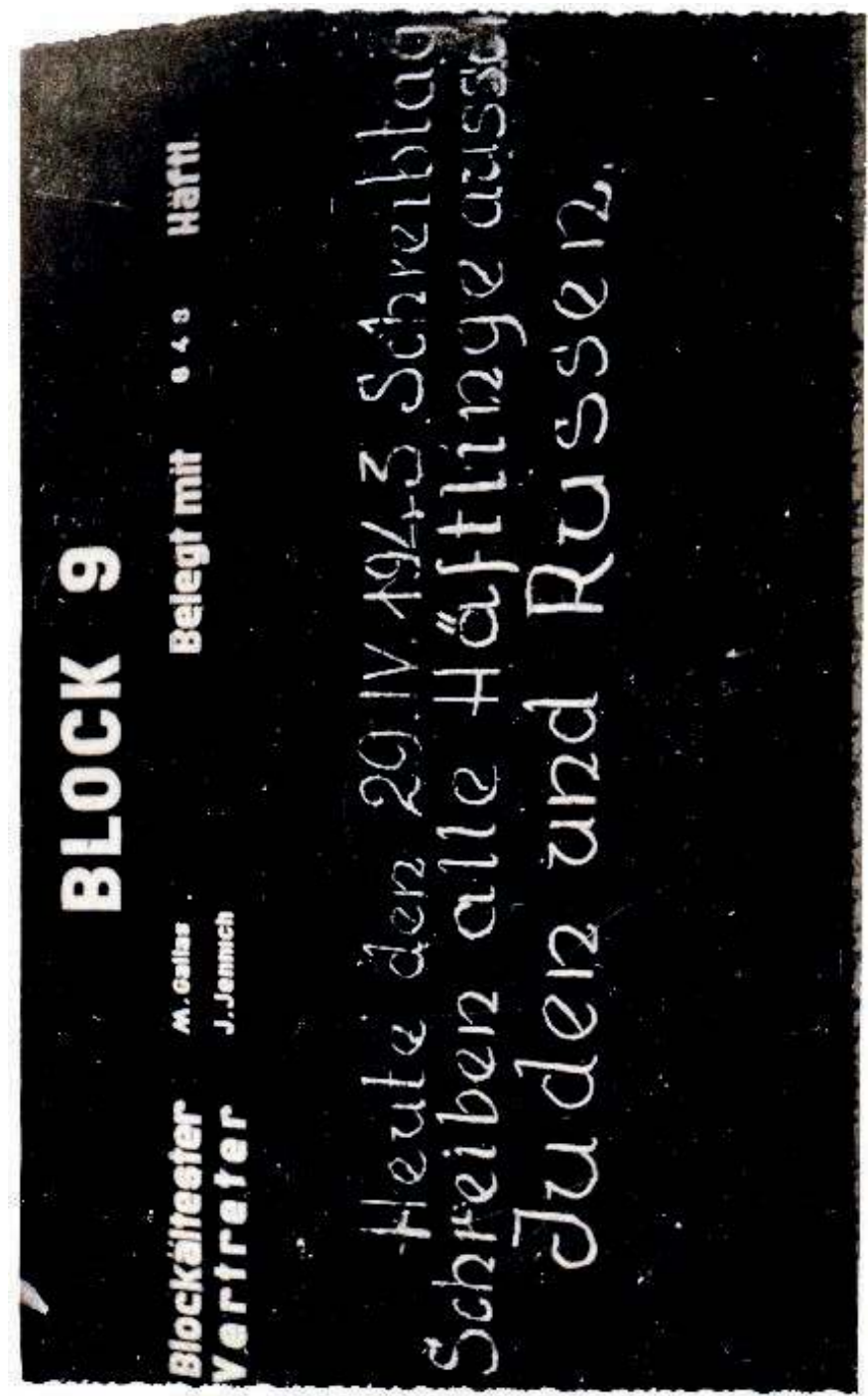

Abbildung 12: Konzentrationslager Auschwitz, Schreibtag für »alle Häftlinge ausser Juden und Russen«. 


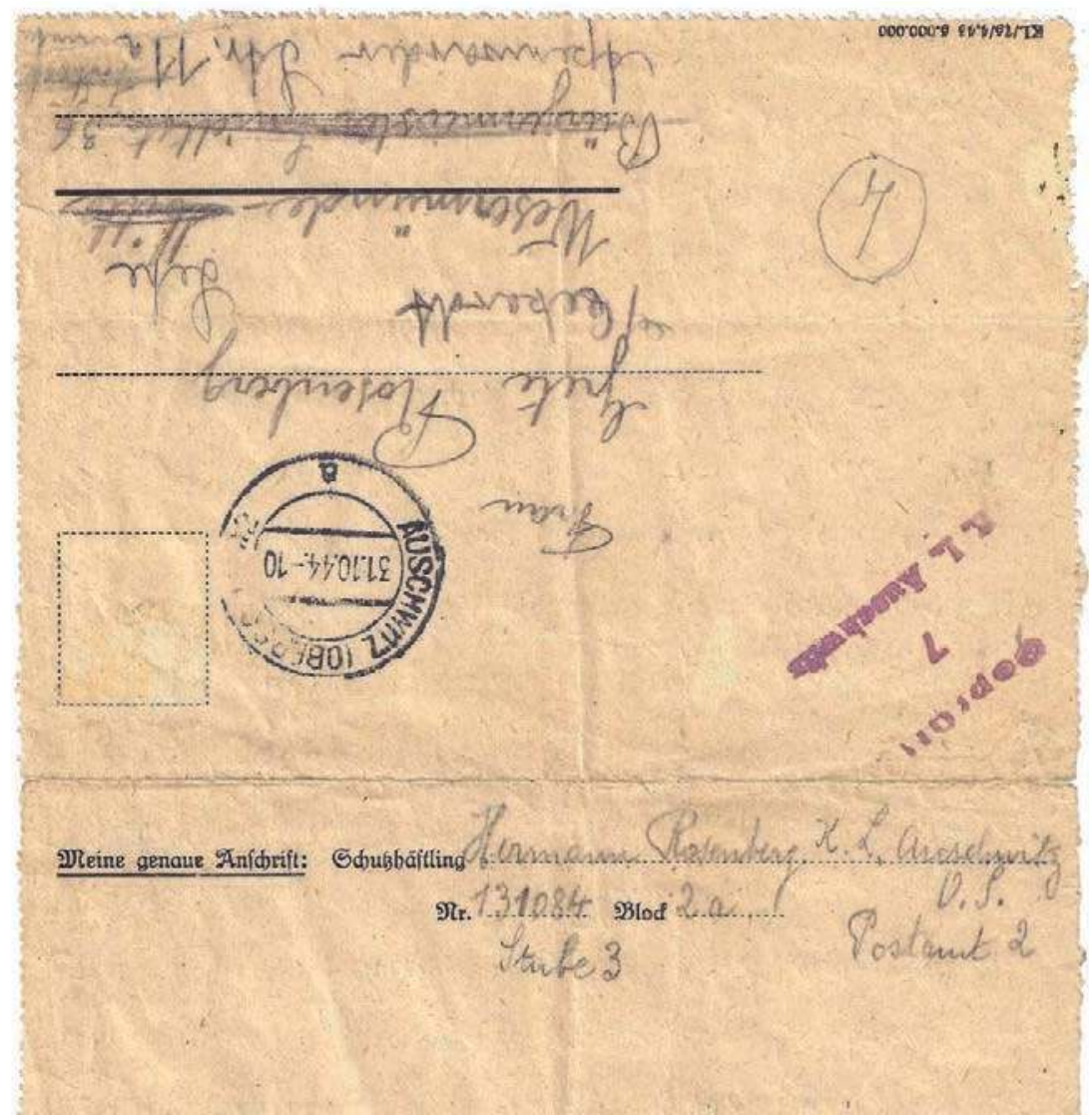

Abbildung 13: Hermann Rosenberg, Faltbrief aus dem Konzentrationslager Auschwitz. 


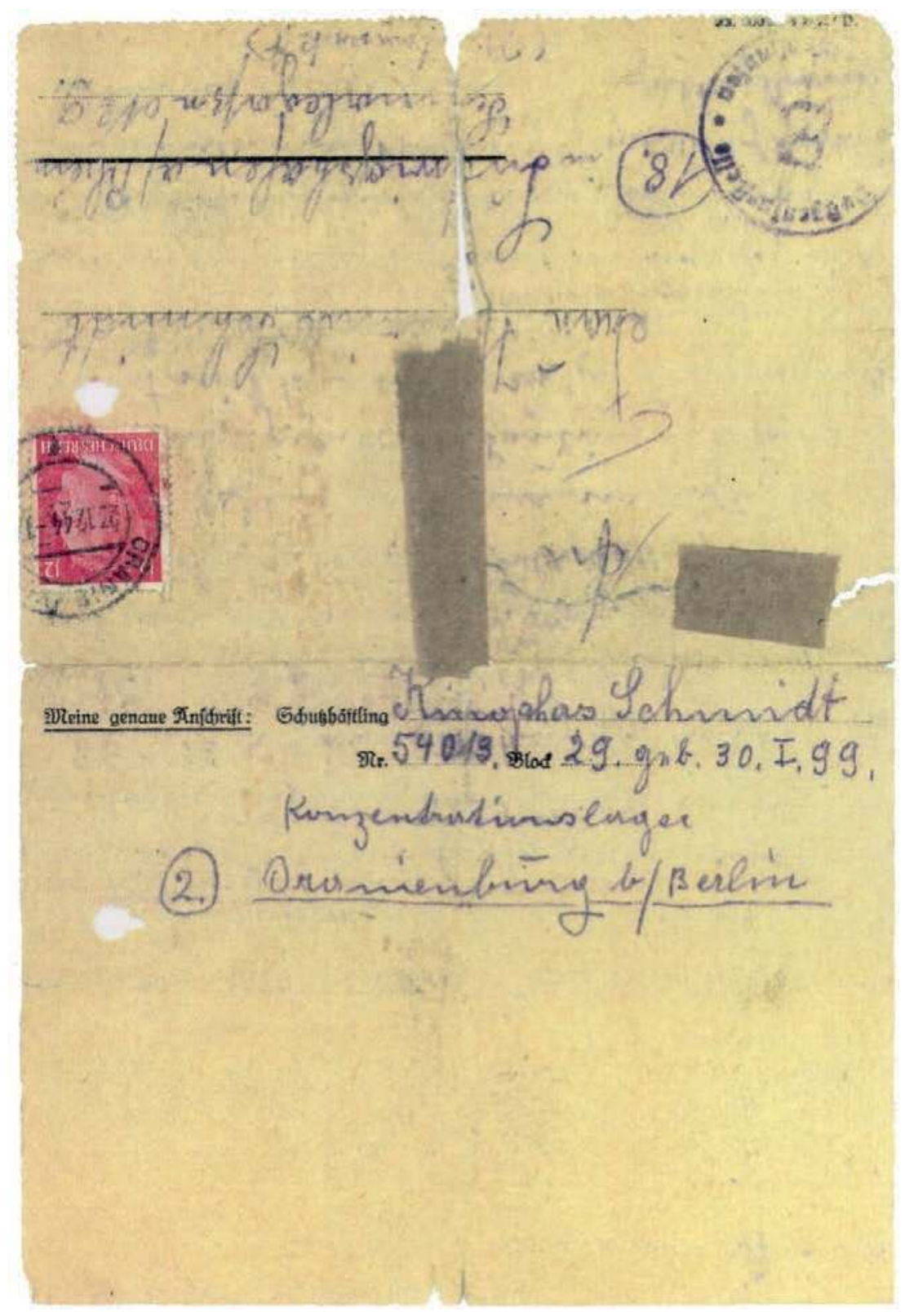

Abbildung 14: Kinophas Schmidt, Faltbrief aus dem Konzentrationslager Sachsenhausen. 


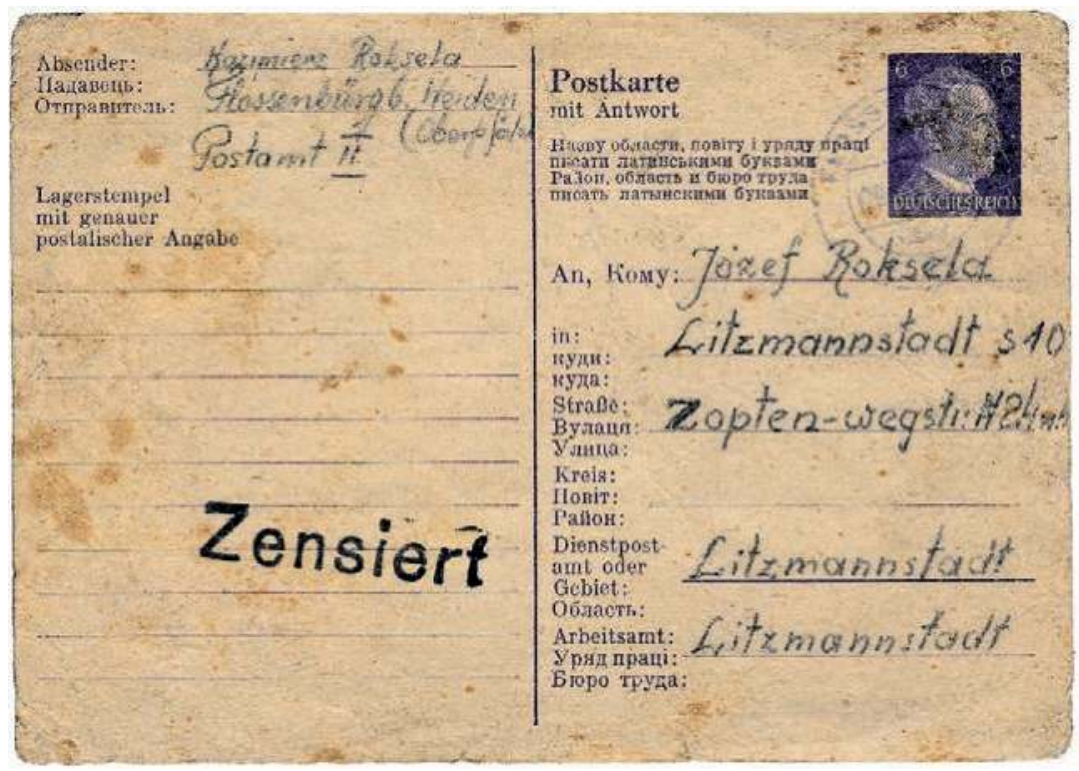

Abbildung 15: Getarnter Absender. Ostarbeiter-Ganzsache des Häftlings Kazimierz Roksela aus dem Konzentrationslager Flossenbürg.

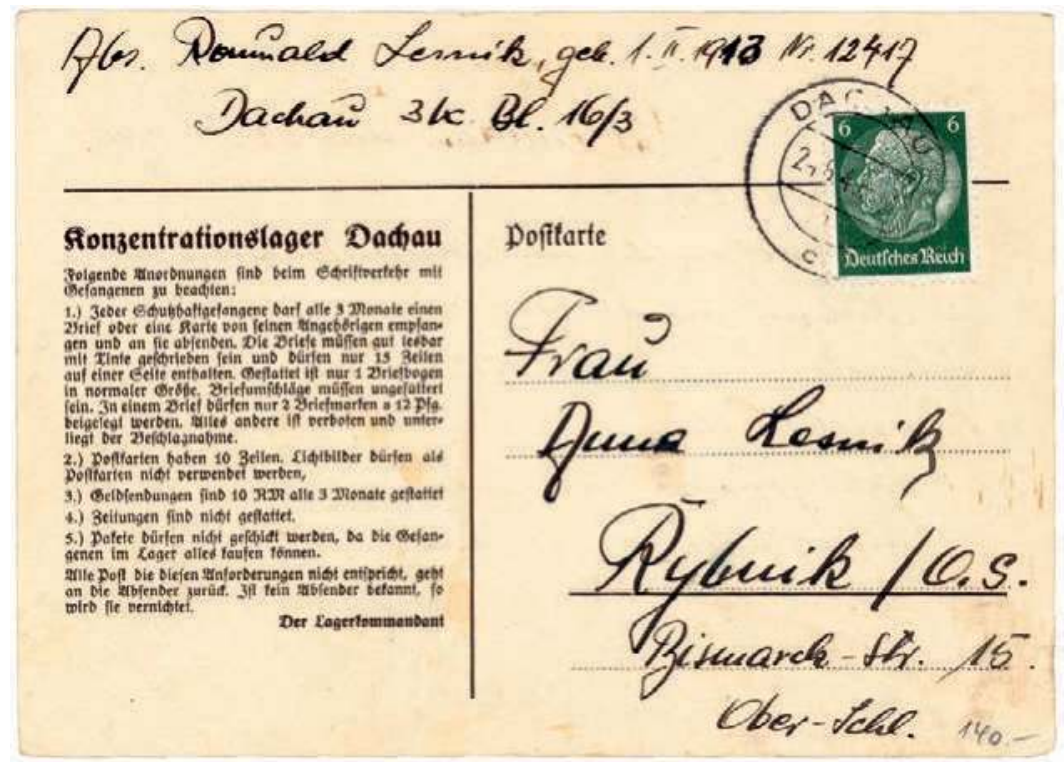

Abbildung 16: Einschränkung der Teilnahme am Postverkehr für Angehörige der Strafkompanie. Vordruck-Postkarte des Häftlings Romuald Lesnik aus dem Konzentrationslager Dachau. 


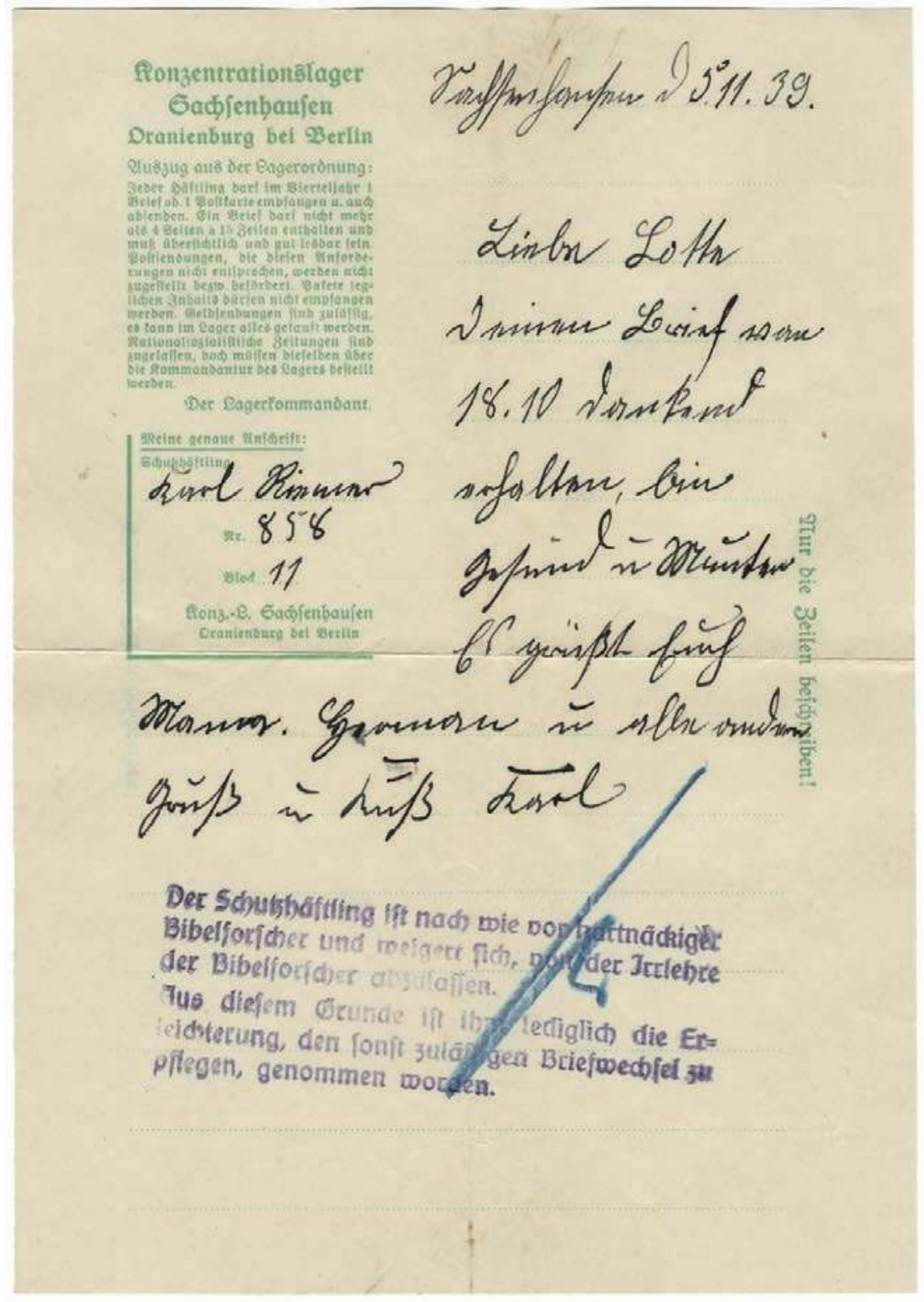

Abbildung 17: Einschränkung der Teilnahme am Postverkehr für Zeugen Jehovas. Faltbrief des Häftlings Karl Riemer aus dem Konzentrationslager Sachsenhausen. 


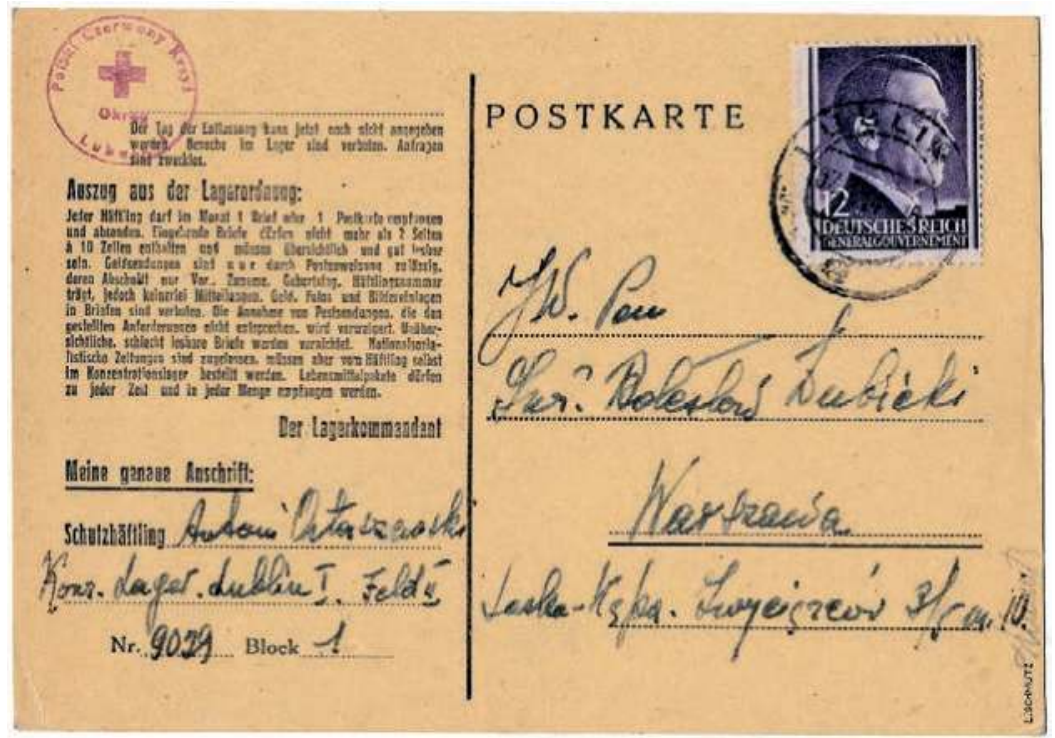

Abbildung 18: Einschränkung der Teilnahme am Postverkehr für Häftlinge des Konzentrationslagers Lublin-Majdanek. Vordruck-Postkarte des polnischen Militärarztes Antoni Ostaszewski. 
Open-Access-Publikation im Sinne der CC-Lizenz BY-NC-ND 4.0 (C) 2021, Vandenhoeck \& Ruprecht GmbH \& Co. KG, Göttingen ISBN Print: 9783847112280 - ISBN E-Lib: 9783737012287 
Konstanze Soch

\section{Ein »verpackter « Konflikt - Die persönliche und politische Dimension des Geschenkpaketverkehrs}

\section{Einleitung}

Es glich einem Ritual. Jedes Mal, wenn Familie Cabus ein Paket aus dem Westen (ein Westpaket) erhielt, öffnete sie es gemeinsam nach dem Abendessen. Dafür wechselte sie von der Küche ins Wohnzimmer - an einen Ort, welcher der Familie für das Auspacken würdig erschien. Zuerst wurde das Paket in die Mitte des großen Wohnzimmertisches gestellt und jeder nahm an diesem Platz. Die Vorfreude stieg, denn das Paket wurde langsam und mit Bedacht ausgepackt. Zuerst die Bänder, dann das Geschenkpapier - schließlich konnte beides noch einmal verwendet werden. Als das Paket dann offen war, füllte sich das Wohnzimmer mit dem so angenehmen Geruch des Westpakets und die bunten Geschenke aus der Bundesrepublik kamen zum Vorschein. ${ }^{1}$

Wie bei Familie Cabus bedeutete es für viele eine große Freude, wenn der Postbote klingelte und ein Westpaket brachte. Diese Geschenksendungen durchbrachen nicht nur symbolisch, sondern auch ganz real die Mauer. Der Duft der Westpakete ist vielen auch heute noch präsent: Es war eine Mischung aus Kaffee, Orangen, Seife und Schokolade.

Doch nicht nur die Menschen aus der Bundesrepublik versandten Päckchen und Pakete über die innerdeutsche Grenze. Auch ihre Verwandten und Bekannten aus der DDR bedachten sie ihrerseits mit Geschenken aus dem Osten (den Ostpaketen). Ebenso wie die Westpakete besaßen auch die Ostpakete einen bestimmten Geruch. Dieser setzte sich vor allem aus selbstgebackenem Stollen und dem Verpackungsmaterial der DDR zusammen.

1 Zit n. Interview Herr Cabus am 7. Januar 2015, 00:30:45. 


\section{Der Beginn des Geschenkpaketverkehrs}

Nach dem Ende des Zweiten Weltkriegs - Deutschland war in vier Besatzungszonen und Berlin in vier Sektoren aufgeteilt - waren Briefe, Päckchen und Pakete die wichtigste Möglichkeit, um den Kontakt mit Verwandten und Bekannten aufrecht zu halten. Doch der Postbetrieb musste in den ersten Wochen und Monaten nach dem Krieg im Sommer 1945 erst wieder aufgebaut werden. Oft waren die Menschen darauf angewiesen, dass Verwandte und Bekannte Briefe und Pakete persönlich überbrachten. Erst zum Jahreswechsel 1945/1946 kam es zur Installation eines zonenübergreifenden Postverkehrs. ${ }^{2}$ Die Hilfe, die sich in Form von Päckchen und Paketen materialisierte, war für die Versender und Empfänger wichtig.

Für jene ersten Jahre nach dem Ende des Zweiten Weltkriegs 1945 war es zunächst weniger der innerdeutsche Päckchen- und Paketverkehr, der den Menschen in Erinnerung geblieben ist, sondern vor allem die sogenannten CARE-Pakete. ${ }^{3}$ Die stets aus stabilem hellbraunem Pappkarton produzierten Pakete mit der Aufschrift »C.A.R.E. - U.S.A.« waren bald der konkretisierte Traum von Freiheit und Wohlstand im grauen Nachkriegsalltag. In kürzester Zeit wusste jedes Kind, dass to care für "sich sorgen « stand. Die meisten Pakete enthielten Corned Beef, Zucker, Kaffee, Schokolade, Honig, Rosinen, Trockenmilch und Eipulver, aber auch für die Deutschen exotisch anmutende Produkte wie Erdnussbutter. Da die Menschen den Amerikanern für die erhaltene Hilfe schnell sehr dankbar waren, entschloss sich die Sowjetunion ebenfalls zu einer solchen Hilfe.

Als Gegenentwurf zum CARE-Paket kam es in der sowjetischen Zone schließlich zur Verteilung sogenannter Pajoks. Da diese jedoch nicht von privaten Spendern finanziert, sondern vom Staat als eine Art Prämie zugeteilt wurden, entwickelte sich in der Bevölkerung schnell die Bezeichnung "Stalinpakete ${ }^{4}$. Auch die Sowjetunion hatte durch die amerikanischen Pakete schnell erkannt, wie wichtig es für die Zufriedenheit der Bevölkerung war, eine Art Zusatzversorgung zur Verfügung zu stellen. Denn gerade nach Kriegsende war es in besonderer Weise die Versorgung mit Lebensmitteln, die für die Menschen von größter Bedeutung war. Ganz anders als die CARE-Pakete stellten die Pajoks allerdings eine Sonderzuteilung dar und sollten Funktionäre, Künstler und »Angehörige der Intelligenz« der Sowjetunion und der sowjetischen

2 Vgl. Jens Ebert: Von der Feldpost zum zivil(isiert)en Briefverkehr, in: www.museumsstiftung.de/briefsammlung/post-von-drueben/briefsammlung-ziviler-briefverkehr.html (letzter Zugriff: 28.11.2015).

3 Vgl. Ebd.

4 Vgl. Ebd. 
Zone gegenüber positiv stimmen, um dort zu bleiben. Auch der Inhalt war ein ganz anderer: Dort waren nicht die vielfach so begehrten Zigaretten, Kaffee oder das beliebte Corned Beef enthalten. So waren die Pajoks zwar einerseits viel größer als ein amerikanisches Paket, andererseits vor allem reichlich gefüllt mit Schmalz, Zucker, Mehl und Kartoffeln. Dass sich der Inhalt von den amerikanischen Paketen so sehr unterschied, erscheint nur folgerichtig, denn in der Sowjetunion hatte der Lebensstandard ein »unvorstellbar niedriges Niveau erreicht $~^{5}$, sodass bereits Grundnahrungsmittel als wahres Geschenk angesehen wurden. Empfänger, die um den Inhalt der CARE-Pakete wussten, teilten diese Einschätzung nicht. Denn die Pajoks lösten eben keine so überwältigende Freude aus wie ihr amerikanisches Pendant. ${ }^{6}$

Trotz aller Systemdifferenzen verfolgte die Installation der beiden Paketarten ein gemeinsames Ziel. Über den Versand mit Lebensmitteln versuchten sowohl die USA als auch die Sowjetunion die unmittelbare Not der ersten Nachkriegsjahre zu lindern und in der Bevölkerung als verlässlicher Partner aufzutreten.

Vor allem nach der Gründung der Bundesrepublik und der DDR im Jahr 1949 kam den Geschenksendungen eine wichtige Funktion zu. Die Bundesrepublik - insbesondere das Bundesministerium für gesamtdeutsche Fragen, das damit beauftragt war, den Willen zur Wiedervereinigung lebendig zu halten mahnte regelmäßig mit den Worten »Trotz Zonengrenze zusammenhalten. Briefe, Päckchen, Pakete nach drüben ${ }^{7}$ oder »Sie warten drüben auf deinen Gruß!! Dein Brief - Dein Päckchen - Dein Paket in die Zone! « ${ }^{8}$, den Paketversand aufrechtzuerhalten. Auf diesem Weg konnte der breiten Öffentlichkeit vermittelt werden, dass die Bundesregierung an der Überzeugung festhielt, dass es nur ein gemeinsames Deutschland geben könne. Gleichzeitig - und dieser Umstand darf zur Zeit des Kalten Krieges nicht zu gering eingeschätzt werden - zeigt die Erforschung der Geschenksendungen sehr deutlich, dass der Inhalt der Päckchen und Pakete zu einem Gradmesser davon wurde, welches wirtschaftliche System das erfolgreichere war. Dies ließ sich in den ersten Jahren am besten an der Versorgung mit Lebensmitteln feststellen, die vor allem zu Beginn ihren Weg in die Päckchen und Pakete fanden. Schnell galt es für die Menschen dies- und jenseits der Grenze als ausgemacht, dass es um die Versorgungslage in der Bundesrepublik wesentlich besser bestellt war. So trug nicht nur die wesentlich frühere Aufhebung der Lebensmittelrationierung in

5 Vgl. Ebd.

6 Vgl. Konstanze Soch: Eine große Freude? Der innerdeutsche Paketverkehr im Kalten Krieg (1949-1989), Frankfurt am Main/New York 2018, S. 77.

7 Staatsarchiv Freiburg, W 124, Nr. 0124, Bild 1.

8 Ebd., Nr. 0121, Bild 1. 
der Bundesrepublik zu diesem Eindruck bei, sondern auch die größere Auswahl an Lebensmitteln.

Auch die DDR warb für den Versand von Geschenksendungen in die Bundesrepublik, allerdings nicht wie im Westen Deutschlands auf einer privaten, sondern auf einer betrieblichen Ebene. So veranlasste die DDR-Führung zu den familiären Hochfesten wie Weihnachten und Ostern stets eine Vielzahl an Volkseigenen Betrieben, Päckchen und Pakete an westdeutsche Arbeiter, die sich in der Bundesrepublik für den Sozialismus einsetzten, zu verschicken. Dies wurde den eigenen Bürgern medienwirksam in Radio, Zeitung und später im Fernsehen vermittelt. In diesem Zusammenhang wurde nicht nur darauf verwiesen, dass es um das Leben der Werktätigen in der Bundesrepublik schlechter bestellt war. Zugleich konnte herausgestellt werden, dass die Versender in der DDR keinen Mangel litten und deshalb umfangreich Hilfe leisten konnten. Schließlich wollte man im "Kalten Paketkrieg " ${ }^{9}$ nicht als Verlierer wahrgenommen werden. Beide Teile, sowohl die DDR als auch die Bundesrepublik, nahmen die Chance wahr, über die karitative Motivation hinaus die politische Funktion der Geschenksendungen zu nutzen. Schließlich ließ sich auf keinem besseren Wege das Bekenntnis vermitteln, von einem geeinten Deutschland überzeugt zu sein. In der konkreten Umsetzung gab es dann jedoch Unterschiede. Während die DDR ihre Hilfe in Form von Päckchen und Paketen nur jenen zukommen lassen wollte, die ihr wohlgesonnen waren, bestand das Ziel der Bundesrepublik darin, möglichst viele Menschen zu unterstützen. Allein durch diesen Ansatz und den Produkten, die es so im Osten nicht zu kaufen gab, gelang es der Bundesrepublik Deutschland, die Sehnsucht nach einem Leben im Westen zu entfachen bzw. auszubauen. Die von Bernd Lindner beschriebene "Sogwirkung des westdeutschen Wirtschaftswunders auf die ostdeutsche Bevölkerung “ ${ }^{10}$ entwickelte sich ungehindert.

Sowohl die BRD als auch die DDR nutzten in den Jahren von 1954 bis 1960 die Ost- und Westpakete als Sendeboten ihres politischen Systems. Die Deutsche Demokratische Republik versuchte vor allem das Bild der »hungernden Brüder und Schwestern" abzustreifen. Vorrangig tat die DDR dies, indem sie kontinuierlich darauf verwies, dass die heimische Wirtschaft nicht nur in der Lage sei, die eigene Bevölkerung zu versorgen, sondern sogar fähig, die »hungernden Arbeiter im Westen" mit Solidaritätspaketen zu bedenken. Dabei spielten der DDR nicht nur die Streiks der westlichen Metallarbeiter Mitte der

9 Konstanze Soch: Eine große Freude? Der innerdeutsche Paketverkehr im Kalten Krieg (19491989), Frankfurt am Main/New York 2018, S. 55.

10 Hier und im Folgenden Jens Ebert: Von der Feldpost zum zivil(isiert)en Briefverkehr, in: www.museums-stiftung.de/briefsammlung/post-von-drueben/briefsammlung-ziviler-briefv erkehr.html (letzter Zugriff: 28.11.2015). 
1950er-Jahre in die Hände, sondern vor allem das Auslaufen der amerikanischen »Eisenhower-Spende«, die den Hilfsorganisationen eine große Anzahl von Lebens- und Genussmittel für den Versand zur Verfügung gestellt hatte. Dies führte unmittelbar zu einem Rückgang der bundesrepublikanischen Versandzahlen und die DDR wurde dadurch in diesen Jahren zum Sieger des "Kalten Paketkriegs". Denn die Menschen aus der DDR verschickten pro Kopf mehr Päckchen und Pakete als die Deutschen westlich der innerdeutschen Grenze.

Dass beide Seiten neben dem Einheitsgedanken überwiegend die wirtschaftliche Hilfe mit dem Päckchen- und Paketversand in Verbindung brachten, verwundert nicht - befanden sich doch beide Systeme im Wettkampf um die Akzeptanz der Menschen. Wie konnte den Bürgern des eigenen Staates eine positive Einstellung besser vermittelt werden als durch einen wirtschaftlichen Aufschwung nach dem Krieg? Dabei war es nicht nur wichtig, die eigenen Bürger von der Wirtschaftskraft des eigenen Landes zu überzeugen. Viel bedeutsamer erschien die Aufgabe, dies dem jeweils anderen Teil Deutschlands $\mathrm{zu}$ verdeutlichen. Besonders die Paketkampagnen der beiden Staaten, welche auf die wirtschaftliche Lage des jeweils anderen eingingen, trugen zu den Vorstellungen vom Gegenüber bei.

Durch die sich stets verändernde Situation des Kalten Kriegs wurde dem Päckchen- und Paketverkehr immer wieder eine politische Bedeutung beigemessen und die Päckchen und Pakete als Sendeboten des jeweiligen wirtschaftlichen Systems verstanden. Aus diesem Grund gerieten sie, verbunden mit der entsprechenden propagandistischen Einbindung in Ost und West, immer wieder in den Fokus der öffentlichen Berichterstattung

\section{Andere Generation, andere Westpakete}

Die Vorbereitungen zum Paketversand sahen in Ost und West unterschiedlich aus. Die Bundesbürger waren sich sicher, welche Produkte im anderen Teil Deutschlands benötigt würden, um eine große Freude zu bereiten. Über die Jahre der Trennung veränderte sich diese Überzeugung bei Teilen der Versender, vor allem bei jenen, die einer neuen, jüngeren Generation angehörten und dadurch andere Schwerpunkte setzten. Die ersten Versender verschickten in erster Linie Lebensmittel. ${ }^{11}$ Das betraf die Geburtenjahrgänge von 1923 bis

11 Auf einen Zeitzeugenaufruf der Autorin zum Thema Päckchen und Paketverkehr zwischen der DDR und der Bundesrepublik meldeten sich mehr als 500 Personen. Auf Basis der Rückmeldungen wurden drei Untersuchungskohorten gebildet: A) die Jahrgänge der um die 1930erJahre Geborenen, die Deutschland als Ganzes erlebten und sich diesem verpflichtet fühlten. 
1935, die ebenso wie ihre ostdeutschen Empfänger den Krieg miterlebt und selbst Mangelerfahrungen gemacht hatten - jene Generation also, die auch noch das vereinigte Deutschland vor dem Krieg kennengelernt hatte. Es wurden insbesondere Lebensmittel verschickt, die ein langes Haltbarkeitsdatum hatten und flexibel verwendet werden konnten. Dazu gehörten in erster Linie Mehl, Zucker, Butter und Fleisch-Konserven, aber auch Kaffee. Um ihr Gegenüber zu erfreuen, verschickten sie auch gerne getragene Kleidung, die noch von guter Qualität war. Jene ersten Versender der Westpakete besaßen durch die gemeinsamen Kriegserfahrungen nicht nur ein hohes Verantwortungsbewusstsein gegenüber ihren >Brüdern und Schwestern` im Osten Deutschlands. Da auch sie mit den Schwierigkeiten des Wiederaufbaus und des Neubeginns zu kämpfen hatten, bedeutete jedes Paket auch eine finanzielle Belastung. Aus diesem Grund konnten sie zu Beginn meist nur kleine Päckchen mit etwas Kaffee und wenigen Lebensmitteln versenden. Der Blick auf Sonderangebote gehörte bei ihnen zum Alltag. Die Verwandten und Bekannten deshalb aber nicht mit einem Geschenkpaket zu bedenken, kam nicht in Betracht. Dem einmal ausgewählten Inhalt blieben sie meist treu und es entwickelte sich eine Art Standardpaket ${ }^{12}$.

\footnotetext{
Diese Menschen waren unmittelbar nach dem Krieg die ersten Päckchenversender. Sie fühlten sich ihren Verwandten und Bekannten stark verbunden. Durch die Rückmeldung der Zeitzeugen umfasste die Gruppe schließlich die Jahre 1923 bis 1935. B) Die um 1949 sowie Anfang/Mitte der 1950er-Jahre Geborenen. Sie waren die ersten Jahrgänge, die ausschließlich in der Bundesrepublik oder DDR sozialisiert wurden und unmittelbar den Mauerbau und die damit verbundene Trennung Deutschlands erlebten. Konkret umfasste die Kohorte die Jahrgänge 1947 bis 1955. C) Auf beiden Seiten gehörten für die in den 1960er-Jahren geborenen Menschen die Teilung Deutschlands und die Existenz der Mauer zum Alltag. Mit dem Transitabkommen Anfang der 1970er-Jahre und der Möglichkeit, das sandere Deutschland kennenzulernen, erfolgte eine erste Annäherung. Im Osten Deutschlands wünschten sich die Päckchenempfänger vor allem die (Marken-)Produkte, die ihnen aus der Werbung bekannt waren. Diesen Sozialisationserfahrungen wurden die Jahrgänge 1959 bis 1971 zugeordnet. In das Sample wurden letztendlich pro Kohorte zwölf Personen aufgenommen. Dabei wurde berücksichtigt, dass einzelne Interviews nicht wie geplant stattfinden können oder es nicht gelingt, eine selbstständige Erzählung zu generieren. Da es dem Anspruch dieses hier beschriebenen Projektes entspricht, Ost- und Westdeutschland gleichbedeutend in die Analyse einzubeziehen, wurde insgesamt eine Balance zwischen Ost- und Westpaketversendern angestrebt. Bei den Interviews handelte es sich nicht im klassischen Sinn um lebensgeschichtlich geführte OralHistory-Interviews, sondern um thematische Interviews mit einem Fokus auf den Päckchenund Paketverkehr, welche die Zeitzeugen je nach Erinnerung mit ihren biografischen Beschreibungen ergänzten. Die Erinnerungen der Zeitzeugen wurden mit Hilfe eines thematischen Interviews erhoben, welches durch einen Leitfaden ergänzt wurde.

12 Jens Ebert: Von der Feldpost zum zivil(isiert)en Briefverkehr. Dies erinnert auch Frau Bleck im Interview am 8. Dezember 2014.
} 
Schon die zweite Versender-Generation handelte anders. Sie waren zwischen 1947 und 1955 geboren und die ersten Jahrgänge, die ausschließlich in der DDR beziehungsweise in der Bundesrepublik sozialisiert worden waren. Den Mauerbau und die Teilung Deutschlands haben sie unmittelbar miterlebt. Diese Menschen griffen nicht mehr ausschließlich zu Grundnahrungsmitteln, sondern ergänzten den Inhalt für ihre Verwandten in Ostdeutschland vor allem durch Genussmittel, Drogerieartikel und neuwertige Kleidungsstücke. Zudem erfragten sie viel häufiger die Wünsche ihres Gegenübers und waren durch die Verbesserung der bundesrepublikanischen Wirtschaftslage in der Lage, etwas mehr Geld für die Geschenkpakete auszugeben. Der Griff zu Markenprodukten wie Jacobs-Kaffee oder Milka-Schokolade gehörte für sie dazu.

Die ersten Versender hingegen hatten dies auf Grund der finanziellen Belastung meist noch vermieden. Zudem empfanden sie es als unangebracht, wenn sich Verwandte und Freunde aus der DDR Markenprodukte wünschten. Häufig stellten sie sich die Frage, warum es nicht auch das No-Name-Produkt sein könnte. Dass in der DDR jedoch nicht bekannt war, dass es eben solche Produkte gab, wussten sie wiederum nicht. Schließlich leisteten sich nur die großen Marken Werbung in Fernsehen und Radio. Deshalb hatten sie als Versender oft das Gefühl, ihr Gegenüber würde ihre Lebenssituation mit einem »Schlaraffenland $\aleph^{13}$ gleichsetzen - ihrer Meinung nach eine Fehleinschätzung.

Die Versender der zweiten Generation hingegen griffen gerne zu Genussmitteln und Markenprodukten, weil sie so die Empfänger im Osten an der bunten Warenvielfalt teilhaben lassen konnten. ${ }^{14}$ Ähnlich gestaltete sich dies auch bei der dritten Gruppe von Versendern, den in den 1960er-Jahren Geborenen. Die Mauer und die Teilung Deutschlands gehörten für sie zum Alltag. Neben dem Standardpaket, welches sich in jeder Familie auf die ein oder andere Art und Weise herausgebildet hatte, erfüllten auch sie gerne Wünsche nach speziellen Kleidungsstücken oder technischen Geräten. ${ }^{15}$ Grundsätzlich blieben sie aber bei der Inhaltsauswahl der Generation ihrer Eltern treu, mit denen sie als Kinder oft zusammen die Westpakete gepackt hatten. ${ }^{16}$

Doch nicht nur der Inhalt veränderte sich im Laufe der Zeit. Zu Beginn des Versandes wurden neben Verwandten und Bekannten oft auch unbekannte Personen bedacht. ${ }^{17}$ Denn besonders zu Beginn des Päckchen- und Paketver-

13 Jens Ebert: Von der Feldpost zum zivil(isiert)en Briefverkehr.

14 Vgl. Interview Frau Herberger am 25. Januar 2015.

15 Vgl. Interview Herr Knechtel am 4. Dezember 2014.

16 Vgl. Interview Frau Kahl am 18. Februar 2015.

17 Rainer Gries: »Jacobs Kaffee - wunderbar« - Päckchen nach drüben: Eine geniale Verbindung von Produktwerbung und Politikpropaganda, in: Mechthild Blum und Thomas Nesseler 
kehrs existierten viele Vereine, die Adressen von DDR-Bürgern an spendenbereite Paten in der Bundesrepublik vermittelten. ${ }^{18}$ Diese Initiativen nahmen mit dem Fortschreiten der Teilung ab.

\section{Der Wunsch nach sAugenhöher der Ostpakete}

Doch mit der bunten Warenwelt, die durch die Westpakete Einzug in die ostdeutschen Wohnstuben hielt, ist nur eine Seite des innerdeutschen Päckchenund Paketverkehrs beschrieben. Er endete an dieser Stelle nicht. Denn den Beschenkten in der DDR war es ebenso wichtig, mit ihren Verwandten und Bekannten in der Bundesrepublik in Kontakt zu bleiben und ihnen mit ihren Geschenksendungen in den Westen eine Freude zu machen. In allen drei Generationen sahen sich die Versender der Ostpakete vor die gleiche Herausforderung gestellt, sodass keine weitere Unterteilung vorgenommen werden kann, nämlich: Was kann man jemandem schenken, der alles zu haben scheint und sich jederzeit im vermeintlich "goldenen Westen ${ }^{19}$ alle Wünsche erfüllen kann? Aus diesem Dilemma heraus entschieden sich die Menschen im Osten Deutschlands, vor allem kunstgewerbliche Gegenstände und regionale Spezialitäten zu verschicken. Aber auch Kalender und belletristische Literatur waren beliebt - konnten diese in der Bundesrepublik doch nur zu vielfach höheren Preisen erworben werden. Auch wenn diese Geschenke in der DDR zu einem niedrigeren Preis angeboten wurden, mussten dafür oft Kontakte genutzt werden, denn die potenziellen Geschenke standen in viel geringerer Anzahl zur Verfügung als in der Bundesrepublik.

Die Mühen, die sich die Menschen im Osten machten, nahmen unterschiedliche Gestalt an. Zum einen drückte sich dies darin aus, dass für viele Geschenke, die als adäquat für den Versand in den Westen erachtet wurden, oftmals lange Zeit angestanden werden musste oder sie nur über Kontakte und Tauschgeschäfte zu erwerben waren. Denn anders als Produkte aus der Bundesrepublik, die zumeist ohne Probleme überall gekauft werden konnten, stellten die Geschenke aus der DDR auch für die Versender eine Rarität dar. Zum anderen bestand ein hoher Aufwand darin, kunstgewerbliche Geschenke selbst

(Hrsg.): Deutschland, einig Vaterland? Geschichte(n), Probleme und Perspektiven, Bonn 1992, S. 80-85, hier S. 82.

18 Vgl. Hilfsring e.V. 1953-1973, Broschüre des Frankfurter Hilfsrings, Frankfurt a. M. o.D. (1973), S. 7.

19 Vgl. Familie M. an Familie K. am 19.04.1980; Museumsstiftung Post und Telekommunikation 3.2011.410. 
herzustellen, sich dafür die Zeit zu nehmen und die entsprechend benötigen Materialien zu besorgen.

Nicht selten stellten die Versender aus dem Osten bei Besuchen in der Bundesrepublik jedoch fest, dass ihr Geschenk trotz der Mühen nicht den beabsichtigten Stellenwert einnahm. So erinnert sich eine Zeitzeugin daran, mit viel Liebe Taschentücher umhäkelt zu haben. Allein das entsprechende Garn zu besorgen, war eine Herausforderung. Als sie zu Besuch in der Bundesrepublik war, stellte sie in einem großen Kaufhaus fest, dass es umhäkelte Taschentücher dort im Doppelpack zu einem sehr günstigen Preis zu kaufen gab. ${ }^{20}$ Ähnlich erging es einer anderen Zeitzeugin. Zur Verlobung des Bruders, der in der Bundesrepublik lebte, hatte sich die Familie lange Zeit Gedanken darüber gemacht, mit welchem Geschenk sie das junge Paar erfreuen könnte. Die Wahl fiel schließlich auf eine Kupferschale. Auch diese Familie musste bei einem Besuch in der Bundesrepublik feststellen, dass das Geschenk wohl keine Freude ausgelöst hatte - verwendeten der Sohn und die Schwiegertochter die Kupferschale doch als Blumenuntersetzer. ${ }^{21}$ Die Enttäuschung darüber war groß.

Die Vorstellung, mit den eigenen Paketen ebenfalls eine Freude bereiten zu können, war schwer zu realisieren und konnte nicht immer eingelöst werden. Der Wunsch, sich auf diesem Wege für die erhaltenen Produkte auf Augenhöhe zu bedanken, blieb eine Herausforderung. Vielen Menschen im Osten Deutschlands war es ein wichtiges Anliegen, mit den Paketen selbst etwas verschenken zu können und nicht ausschließlich als Nehmende wahrgenommen zu werden. Wie sehr hätten sie sich darüber gefreut, wenn ihre Freunde und Verwandten im westlichen Teil des Landes einen Wunsch geäußert hätten. Doch dies geschah in der Regel nur selten - erlaubten es sich die Bundesbürger doch meistens nicht, gegenüber den vermeintlich ärmeren Empfängern aus der DDR Wünsche zu artikulieren. Und selbst wenn Wünsche geäußert wurden, bestand weiterhin die Unklarheit darüber, ob die ausgewählten Gegenstände wirklich zur Freude im Westen beitrugen, denn die Rückmeldungen auf den Inhalt der Ostpakte waren meist nicht umfangreich. So blieben die Versender erneut verunsichert zurück. ${ }^{22}$

20 Vgl. Interview Frau Förster am 5. Februar 2015.

21 Vgl. Interview Frau Dilling am 17. Februar 2015.

22 Vgl. Interview Frau Winterfeld am 4. Februar 2015. 


\section{Die Kontrollen auf beiden Seiten der Mauer}

Auf beiden Seiten erfuhr der Geschenkpaketversand mit den durchgeführten Kontrollen eine Beeinträchtigung. Dabei ist zu betonen, dass eben nicht nur die DDR, sondern auch die Bundesrepublik den privaten Päckchen- und Paketverkehr kontrollierte. ${ }^{23}$ Beiden deutschen Staaten war dabei gemeinsam, dass sie die Geschenksendungen überprüften, um mögliche Agenten und Spione zu enttarnen. Die DDR bezeichnete diese im offiziellen Sprachgebrauch als 'Schieber` und `Spekulanten` und gab in ihren veröffentlichten Berichterstattungen an, diese vor allem zu verfolgen, um wirtschaftlichen Schaden von der DDR fernzuhalten. Auch sie hatte während des Kalten Kriegs ein politisches Interesse daran, eine mögliche Infiltration zu vermeiden. Doch ganz im Unterschied zur BRD bestand zudem ein großes wirtschaftliches Interesse an der Überprüfung der Päckchen und Pakete. Denn die DDR fürchtete um die Sogwirkung der Westpakete auf ihre Bürger ${ }^{24}$ - und dies nicht zu unrecht. Schließlich enthielten die Geschenksendungen vor allem Produkte, die es in der DDR selbst nicht zu kaufen gab. So entstand für die Empfänger in der DDR unmittelbar der Eindruck, dass es um das Leben in der Bundesrepublik besser bestellt sei.

Besonders zu Beginn der Nachkriegszeit wurde die Versorgung mit Lebensmitteln von den Menschen als Gradmesser in Bezug auf die wirtschaftliche Situation des eigenen Landes betrachtet. Aus diesem Grund verwundert es kaum, dass sich die DDR 1954 dazu entschloss, die sogenannte »Verordnung über den Geschenkpaket- und -päckchenverkehr auf dem Postwege mit Westdeutschland, Westberlin und dem Ausland " (GVO) zu erlassen. ${ }^{25}$ Die schon vorher existierenden Bestimmungen wurden auf diesem Wege zusammengefasst und erheblich erweitert. Zum ersten Mal wurde nun der Begriff der Geschenksendung im Sinne des Gesetzes umfassend definiert. So waren diese als "unentgeltliche Zuwendungen, die unmittelbar von einem privaten Absender (natürliche Person) an einen privaten Empfänger (natürliche Person) aufgrund persönlicher Beziehungen zum persönlichen Verbrauch oder Gebrauch « ${ }^{26}$ verschickt wurden, zu verstehen. Die GVO beschrieb zudem umfassend, wel-

23 Vgl. Josef Foschepoth: Überwachtes Deutschland. Post- und Telefonüberwachung in der alten Bundesrepublik, Göttingen 2013.

24 Vgl. Bernd Lindner: »Dein Päckchen nach drüben«. Der deutsch-deutsche Paketversand und seine Rahmenbedingungen, in: Christian Härtel, Petra Kabus (Hrsg.): Das Westpaket: Geschenksendung, keine Handelsware, Berlin 2000, S. 25-44, hier S. 26.

25 Vgl. Jörn-Michael Goll: Innenansichten. Die Kontrolle des deutsch-deutschen Paket- und Päckchenverkehrs durch Zoll und Staatssicherheit in der DDR, in: DA, 40 (2007) 6, S. 1026.

26 Büro des Ministerrates der Deutschen Demokratischen Republik (Hrsg.): Gesetzesblatt der Deutschen Demokratischen Republik, Teil 1, 74/1954, Berlin 20.08.1954, S. 727. 
che Lebensmittel und welche Gegenstände in welcher Menge grundsätzlich in die DDR eingeführt werden durften. Ebenso wichtig erschien es der DDRFührung, nicht nur die Einfuhr zu reglementieren, sondern auch die Ausfuhr zu beschränken. Zum einen, damit wichtige Erzeugnisse aus der eigenen Produktion nicht dem eigenen Markt entzogen wurden. Zum anderen, damit Menschen, die gewillt waren die DDR zu verlassen, über Geschenksendungen nicht ihre Flucht vorbereiten konnten. Diese Gründe kommunizierte die DDR in der Presse umfangreich und immer unter der Prämisse des Schutzgedankens für das eigene Land.

Die Meinung der DDR-Regierung, dass die GVO für »die Pflege der Beziehungen der Menschen untereinander als sehr großzügig betracht[et] « ${ }^{27}$ werden müsse, teilten die Menschen sowohl im Westen als auch im Osten Deutschlands nicht. Die Presse in der Bundesrepublik titelte »Päckchen durchbrechen den Eisernen Vorhang « ${ }^{28}$, »Für Mutters Konserven gesperrt ${ }^{29}$ und »Pankow kontrolliert rigoros Geschenkpakete « ${ }^{30}$. Die DDR-Bevölkerung ihrerseits ging mit der Geschenkverordnung ebenfalls nicht konform. Abzulesen ist dies an der Vielzahl der Eingaben, die in den Archiven zu Zehntausenden vorhanden sind. Nach dem Mauerbau 1961 wurden die Bestimmungen der GVO deutlich verschärft. Doch der Päckchen- und Paketverkehr erfuhr über Jahre nicht ausschließlich strengere Sanktionierungen. Als Ende der 1970er-Jahre die Weltmarktpreise für Kaffee extrem anstiegen, entschied sich die DDR dazu, alle Einfuhrschranken für Kaffee, Tabakwaren, Spirituosen, Sekt und Wein fallen zu lassen. ${ }^{31}$ Der Unmut der eigenen Bevölkerung sollte so abgedämpft werden.

Anders als die DDR, die die Kontrollen mit dem Schutzgedanken zu rechtfertigen versuchte, vermied es die Bundesrepublik um jeden Preis, die Öffentlichkeit erfahren zu lassen, dass auch sie die Päckchen und Pakete kontrollierte. Schließlich würde dies im Widerspruch zu der stets formulierten Empörung über die Kontrollen durch die DDR stehen. Denn auch in der Bundesrepublik stellte die Kontrolle eine klare Verletzung des Grundgesetzes dar. ${ }^{32}$ Intern rangen die dortigen Politiker um das richtige Vorgehen und die Aufweichung des Grundgesetzes. Die diffuse Angst, im Kalten Krieg durch das Unterlassen

27 Zit. n. BArch Berlin, DL 203/2575, unpag.

28 Zit. n. Münchner Merkur, 21. August 1958.

29 Zit. n. Lübecker Nachrichten, 16. März 1955.

30 Zit. n. Wiesbadener Kurier, 17. März 1955.

31 Vgl. Christian Härtel: Ostdeutsche Bestimmungen für den Paketverkehr im Spiegel westdeutscher Merkblätter, in: Christian Härtel, Petra Kabus (Hrsg.): Das Westpaket: Geschenksendung, keine Handelsware, Berlin 2000, S. 45-56, hier S. 52.

32 Vgl. Josef Foschepoth: Überwachtes Deutschland, S. 108. 
von Handlungen jedoch einen wirtschaftlichen oder politischen Schaden davonzutragen, überwog, sodass es letztendlich dabei blieb, dass auch private Sendungen überprüft wurden und die Öffentlichkeit uninformiert war. ${ }^{33}$ Vor allem der Deutschen Bundespost widerstrebten die neuen Anforderungen - war sie sich als ausführendes Organ doch unsicher darüber, ob dieser Eingriff »nicht schon wieder eine massive Verletzung geltenden Rechtes war ${ }^{34}$. Aus diesem Grund führte die Bundespost - ganz zum Missfallen des Bundesinnenministeriums und des Zolls - diese Aufgabe nicht mit höchster Priorität aus. In der Wahrnehmung der Menschen `hüben` wie `drüben` war es jedoch ausschließlich die DDR, welche ihre privaten Geschenke, deren Inhalt sie mit viel Liebe ausgesucht und verpackt hatten, kontrollierte und beschlagnahmte. Aus diesem Grund gaben sich die Kontrolleure der Bundesrepublik beim Wiederverpacken der Geschenksendungen meist nicht allzu viel Mühe ${ }^{35}$ - dachten die Menschen doch ohnehin, dass die Beschädigungen bei den DDR-Kontrollen entstanden seien.

Trotz der detaillierten Beschreibungen der GVO kam es immer wieder zu Schwierigkeiten für die Versender. Zum einen, da die Regelungen einem stetigen Wandel unterlagen und so die Versender teilweise nicht über die jeweils aktuell erlaubten Höchstmengen informiert waren. Dadurch kam es häufig zu einem ungewollten Verstoß gegen die GVO, was wiederum zu einer Teileinziehung oder Beschlagnahmung führte. Zum anderen entstanden immer wieder Probleme, da ein Teil des Inhalts - trotz genauer Befolgung der gesetzlichen Bestimmungen - beim Empfänger nicht mehr in der Geschenksendung vorzufinden war. Dies lag an den teilweise bewusst unscharf formulierten Bestimmungen der GVO, damit der Zoll der DDR bei seinen Überprüfungen stets einen gewissen Spielraum hatte. So durften beispielsweise "Schundliteratur « ${ }^{36}$ und $»$ Hetzmaterial $\aleph^{37}$ nicht in Päckchen und Paketen enthalten sein. Was sich jedoch genau hinter diesen beiden Begriffen verbarg, blieb unklar und variierte auch in der Vorstellung der DDR-Kontrolleure. So konnte es ohne weiteres passieren, dass ein und dasselbe Buch in Leipzig aus der Geschenksendung entfernt wurde, während es in Erfurt die innerdeutsche Grenze passierte. Eine Entnahme konnte geschehen, weil sich einige Kontrolleure persönlich berei-

\footnotetext{
33 Vgl. Ebd., 111.

34 Zit. n. Ebd. S. 108.

35 Vgl. Erinnerungsprotokoll Daniel Schmidt, Mitarbeiter eines westdeutschen Sicherheitsunternehmens, Interview am 24. März 2015.

36 Jens Ebert: Von der Feldpost zum zivil(isiert)en Briefverkehr.

$37 \mathrm{Vgl}$. Ebd.
} 
cherten oder die Kommunikation zwischen Versendern und Empfängern bewusst gestört werden sollte. ${ }^{38}$

Trotz der intensiven Bemühungen seitens der DDR, den Versand der Geschenksendungen einzuschränken, war sich die DDR-Regierung darüber bewusst, dass sie auf den Inhalt der Westpakete angewiesen war. Eine Studie des Marktforschungsinstitutes der DDR aus dem Jahr 1978 zeigt, wie groß der Umfang der erhaltenen Lebensmitteln war, die die DDR erreichten: ${ }^{39}$

Kaffee
Schokolade und Schokoladenerzeugnisse
Südfrüchte und Obst
Rosinen, Mandeln, Nüsse, Kokosflocken
Bekleidung für Damen, Herren und Kinder
Damenstrumpfhosen
Seife, Waschmittel und Rasierklingen
$1.596,6 \mathrm{t}$
$1.156,0 \mathrm{t}$
$799,7 \mathrm{t}$
$112,8 \mathrm{t}$
2,8 Mio. Stück
2,3 Mio. Stück
4,6 Mio. Stück

Die ideologische Bedrohung, die den Westpaketen durch die DDR zugeschrieben wurde, überwog nicht die Tatsache, dass sich durch die Einfuhr der Lebensmittel in den Päckchen und Paketen in erheblichem Maße Devisen einsparen ließen. Denn jedes Lebensmittel, welches die DDR auf dem Postweg erreichte, musste nicht eigens teuer eingeführt werden. So stellten die Geschenksendungen für die DDR stets eine Gratwanderung und ein Abwägen zwischen wirtschaftlichen Interessen und politischen Vorstellungen dar. Dieses Ringen zeigte auch die Kaffee-Krise Ende der 1970er-Jahre. Durch Missernten stieg der Kaffeepreis um das Sechsfache an. ${ }^{40} \mathrm{Da}$ eine mögliche Steigerung der Ausgaben im Fünfjahresplan jedoch nicht vorgesehen war, bestand keine Möglichkeit, die neuen Preise zahlen zu können. Nachdem eine Streckung des Kaffees mit Ersatzstoffen unter großem Protest der Bevölkerung gescheitert war, ließ die DDR im September 1977 alle bisher bestehenden Einfuhrbeschränkungen für Kaffee, Tabakwaren, Spirituosen, Wein und Sekt fallen. Zu groß erschien der DDR die Gefahr, dass der Unmut der Bevölkerung weiter anschwoll. ${ }^{41}$

38 Vgl. Schlussbericht über eine Verurteilung, 30. Juni 1988, Bundesbeauftragter für die Unterlagen des Staatssicherheitsdiensts (BStU), MfS, HA IX, Nr. 4635, S. 9.

39 Vgl. BStU, MfS, ZAIG, 15044, in: Christine André, Lilo Nagengast: Öffnen oder weiterleiten? Die Paketkontrolle in der DDR, in: Ein offenes Geheimnis. Post- und Telefonkontrolle in der DDR (Katalog der Museumsstiftung Post und Telekommunikation, Bd. 13), Heidelberg 2002, S. 171.

40 Vgl. Monika Sigmund: Genuss als Politikum. Kaffeekonsum in beiden deutschen Staaten (Studien zur Zeitgeschichte Bd. 87), Berlin/München/Boston 2015, S. 202.

41 Vgl. Ebd., S. 266. 


\section{Der Widerstand und die Folgen der Kontrollen}

Die Menschen sowohl in der Bundesrepublik als auch in der DDR wandten sich bei Verlust ihrer Sendungen, Teileinziehungen oder Beschädigungen oft mit Eingaben an die ihrer Meinung nach zuständige Stelle. Dies waren auf Seiten der DDR das Ministerium für Post- und Telekommunikation sowie die Zollverwaltung der DDR und in der Bundesrepublik die Bundespost und das Bundespräsidialamt. In ihren Eingaben verdeutlichten die Betroffenen ihren Unmut und versuchten durch die ausführliche Beschreibung ihrer eigenen Lebenssituation, aber auch der der jeweiligen Empfänger zu verdeutlichen, weshalb der Versand ihrer Päckchen und Pakete so wichtig sei. Die in den Eingaben vorgenommenen Beschreibungen ähnelten sich dabei in mehreren Punkten. So begann die Argumentation zumeist mit dem Verweis auf die gesundheitliche Verfassung. Es war nicht nur die Eigenbeschreibung der ostdeutschen Empfänger, sich in einem »sehr schlechten Gesundheitszustands ${ }^{42} \mathrm{zu}$ befinden, sondern auch die Fremdbeschreibung der westdeutschen Versender über die Situation der Empfänger, die sich »krank und elend « ${ }^{43}$ fühlen würden und aus diesem Grund einer Unterstützung bedürften, die sich regelmäßig in den Beschwerdebriefen wiederfanden. Zudem argumentierten beide Seiten, dass aufgrund der finanziellen Lage, die sich durch die Anzahl der Familienmitglieder oder die aktuelle Familiensituation ergab, die Pakete wichtig seien. Besonders interessant ist der immer wieder auftauchende Verweis darauf, dass der Versand von Westpaketen für die Menschen in der Bundesrepublik finanziell auch entsprechend bewältigt werden müsse. So schrieb Frau Arndt aus Potsdam, dass »auch die Menschen in West-Berlin [...] ihr Geld schwer erarbeiten [müssten] « ${ }^{44}$. Die Vorstellung, dass jene Leute mit »Glücksgütern ausgestattet ${ }^{45}$ seien, so eine weitere Versenderin, sei grundlegend falsch. Schließlich müssten die westdeutschen Werktätigen genauso hart arbeiten und seien somit »einfache Arbeiter wie wir « ${ }^{46}$, wie Frau Reddel aus Breitenbach betonte. Aus diesem Grund wiege der Verlust von versendeten Geschenksendungen auch hier schwer. ${ }^{47}$

42 Zit. n. Eingabe über den Verbleib eines Westpakets an das Ministerium für Post- und Fernmeldewesen, 9. April 1987, BArch Berlin, DM 3/33633, unpag.

43 Zit. n. Ebd.

44 Zit. n. Eingabe über den Verbleib eines Westpakets an das Ministerium für Post- und Fernmeldewesen, 19. Januar 1981, BArch Berlin, DM 3/33632, unpag.

45 Zit. n. Ebd.

46 Zit. n. Eingabe über den Verbleib eines Westpakets an das Ministerium für Post- und Fernmeldewesen, 22. Oktober 1970, BArch Berlin, DM 3/13625, unpag.

$47 \mathrm{Vgl}$. Bundesministerium für Post- und Telekommunikation über »Geschenksendungen in die SBZ«, 27. Februar 1962, BArch Berlin, DM 3/33633, unpag. 
Damit die Bundesbürger - trotz der vor allem in den 1960er-Jahren stark gestiegenen Zahl der Beschlagnahmungen und Verluste - weiterhin Päckchen und Pakete in die DDR versenden und so zum immer wieder durch die Bundesrepublik beschworenen Zusammenhalt mit den Brüdern und Schwestern im Osten beitragen konnten, entschied die Bundesregierung, die Versender finanziell $\mathrm{zu}$ entschädigen. ${ }^{48} \mathrm{Um}$ von vornherein den Kontrolleuren der DDR wenig Anlass zu geben, etwas an den verschickten Päckchen und Paketen zu beanstanden, bemühte sich die Bundesrepublik zudem, umfangreich über die Versandbestimmungen zu informieren. Dazu verfasste und aktualisierte sie in regelmäßigen Abständen Flug- und Merkblätter sowie eine Vielzahl von Plakataktionen. Mit dem Slogan "Dein Paket nach drüben ${ }^{49}$ versuchte das "Merkblatt für die Geschenksendungen in die Sowjetzone ${ }^{50}$, über die Vorgaben im Bereich der Lebensmittel, Medikamente und Bücher zu informieren und Tipps zu geben. Die Bundesrepublik ließ bei den Versendern der Päckchen und Pakete keinen Zweifel daran aufkommen, dass ihrer Überzeugung nach die Anzahl der Briefe und Geschenke in unmittelbaren Zusammenhang mit dem persönlichen Kontakt der Menschen über die Mauer hinweg stand. Zudem bestand für die Bundesbürger, anders als für die Menschen in der DDR, die Chance, die Ausgaben, die ihnen durch den Versand entstanden, steuerlich geltend zu machen. ${ }^{51}$

Doch nicht immer ließ sich der entstandene Schaden finanziell beheben. Nämlich immer dann, wenn die Versender ihre Verwandten und Bekannten mit einer Geschenksendung überraschen wollten und das Paket vorher nicht angekündigt wurde. Erreichte die Geschenksendung den Empfänger dann nicht, entstand oft eine missliche Situation: Der Versender erhoffte sich eine Bestätigung beziehungsweise einen kleinen Dank. Diese erhielt er jedoch nicht und da der Empfänger seinerseits nicht wusste, dass er mit einem Paket bedacht werden sollte, konnte er keine Rückmeldung über einen etwaigen Verlust geben. Nicht selten waren dann beide Seiten enttäuscht. Die Empfänger, weil die sich über ein Paket zu Weihnachten oder zum Geburtstag gefreut hätten, und die Versender, weil sie wiederum keine Reaktion auf ihr Geschenk erhalten hatten. Ein Missverständnis, welches nicht immer aufgelöst wurde, weil beide Seiten der jeweils anderen nicht zu nahe treten wollten.

48 Vgl. Merkblatt über Geschenksendungen in die sowjetische Zone, März 1960, BArch Koblenz, B 257/22777, unpag.

49 Zit. n. Merkblatt für Geschenksendungen in die sowjetische Zone, März 1960, BArch Koblenz, B 257/21085, unpag.

50 Zit. n. Ebd.

51 Vgl. Frankfurter Allgemeine Zeitung, 11. Dezember 1962. 
Auch Familie Geis wollte den Versendern von Westpaketen durch Nachfragen nicht zu nahe treten. Selbst dann nicht, wenn die Versenderin die eigene Oma war, die bereits in der Bundesrepublik lebte. So wünschte sich der Sohn von seiner Großmutter ein Matchbox-Auto K-19 und war voller Vorfreude, mit diesem bald spielen zu können. Doch der Wunsch des Jungen wurde trotz beharrlichen Wartens nie erfüllt. Obwohl er das Auto so sehr herbeigesehnt hatte, wurde die Oma auf diesen unerfüllten Wunsch nie angesprochen. Das Erkundigen nach Paketen fühlte sich für die Familie wie Betteln an - ein Eindruck, den sie auf jeden Fall vermeiden wollte. ${ }^{52}$ Später dann, als Frau Geis ihre persönlichen Akten bei der Behörde des Bundesbeauftragten einsah, stellte sie fest, dass der Wunsch die Großmutter nie erreicht hatte. Der Originalbrief befand sich in den Akten. Der Versenderin war der Wunsch ihres Enkels also gar nicht bekannt gewesen. ${ }^{53}$

Besonders Personen, die einen Ausreiseantrag gestellt hatten oder dem nichtsozialistischen Ausland gegenüber positiv eingestellt waren, erfuhren eine engmaschige Überprüfung ihrer Post. So wurden nicht nur ihre Briefe gelesen, bei Bedarf einbehalten und die Geschenksendungen überprüft. Die Art und Weise der Überprüfung stand meist in unmittelbaren Zusammenhang mit dem zuständigen Postkontrollamt. Trotz einheitlicher Vorgaben fiel deren Ausgestaltung im Einzelfall unterschiedlich aus. ${ }^{54}$

So wurden die Päckchen und Pakete, die Familie Geis aus dem Westen erhielt, regelmäßig beschädigt. Oft wurden die in den Geschenksendungen enthaltenen Fischdosen - die Verwandtschaft wusste, dass die Familie diese besonders gerne aß - zerstört, um ganz im Sinne der Inhaltsüberprüfung die enthaltenen Lebensmittel durch Öffnung zu kontrollieren. Da in diesen Fällen die Dosen mit Löchern versehen wurden, ergoss sich das Öl der Konservendose meist über den restlichen Inhalt wie Waschmittel, Schokolade oder Kaffee, der somit nicht mehr nutzbar beziehungsweise ungenießbar war. ${ }^{55}$ War auch zuvor schon mal eine Schokoladentafel zerbrochen, nahm die Zerstörung des Paketinhaltes merklich zu, nachdem Frau Geis ihren Ausreiseantrag gestellt hatte. Anscheinend sollte mit allen Mitteln versucht werden, den Kontakt mit den Verwandten und Bekannten in der Bundesrepublik zu stören, um einen Abbruch zu forcieren.

Auch wenn die Kontrolleure auf beiden Seiten angehalten waren, sich große Mühe beim Wiederverpacken zu geben, damit die Kontrolle nicht auffiel, war

52 Vgl. Interview Frau Geis am 24. Februar 2015, 00:36:25.

53 Vgl. Ebd., 00:36:11.

54 Vgl. Interview Herr Kleer am 28. Januar 2015, 00:06:12.

55 Vgl. Interview Frau Geis am 24. Februar 2015, 00:03:54. 
dies für die Betroffenen meist ohne Weiteres festzustellen. ${ }^{56}$ Sie erkannten vor allem an der Art und Weise, wie die Geschenksendungen wieder verpackt beziehungsweise die Briefe verschlossen wurden, eine Überprüfung - entweder, weil der Versender eine ganz bestimmte Art hatte, die Paketschnur zu binden, oder der Brief auf einmal neu verschlossen wirkte. Hatten die Betroffenen in Ost und West den Verdacht, dass sie kontrolliert wurden, nutzen sie häufig die Möglichkeit einer extra filigranen und ausgefallenen Verpackung, sodass eine Zerstörung offenbar wurde.

\section{Die Diebstähle in der DDR}

Da der Unmut der DDR-Bürger über die Diebstähle Anfang der 1980er-Jahre stärker denn je zu Tage trat, beschloss die DDR-Regierung auch ihre Überprüfungen zur "Gewährleistung von Sicherheit und Ordnung in den Abteilungen $\mathrm{M}$ sowie bei der Deutschen Post ${ }^{57} \mathrm{zu}$ intensivieren. Die internen Untersuchungen waren nach Einschätzung des Ministeriums für Staatssicherheit (MfS) von äußerster Wichtigkeit, da die Verluste in hohem Maße zu einem politischen Schaden und Ansehensverlust der DDR und der DDR-Post sowohl in der DDR selbst als auch im Ausland führen würden. ${ }^{58}$ Dabei war nicht der Diebstahl an sich für die DDR-Regierung ein Problem, sondern vielmehr die Berichterstattung darüber. Auffällig in den Akten des MfS ist die Beschreibung, dass zwar der Zoll und das MfS die Geschenksendungen kontrollieren würden, ${ }^{59}$ für Diebstähle jedoch ausschließlich die Mitarbeiter der DDR-Post verantwortlich seien. Diese Annahme zugrunde legend, überrascht es keineswegs, dass sich in den Anklageschriften und Verfahrensakten ausschließlich Mitarbeiter der Post wiederfinden. Dabei ist davon auszugehen, dass es sich hierbei wohl eher um ein politisches Kalkül als eine wahrheitsgetreue Wiedergabe handelt. In allen Personenkreisen, die mit der Kontrolle von Päckchen und Paketen betraut waren, wird es Menschen gegeben haben, die sich persönlich bereicherten. In der Öffentlichkeit sollten allerdings das ganze Ausmaß und der betriebene Aufwand, um die privaten Sendungen zu überprüfen, nicht bekannt werden.

56 Vgl. Interview Herr Siebold am 18. März 2015, 00:49:59.

57 Zit. n. Gewährleistung von Sicherheit und Ordnung in der Abteilung $M$ sowie bei der Deutschen Post, 1980, BStU, MfS, Pdm, Abt. XIX, Nr. 1350, S. 17.

58 Vgl. Information zur Lage im Bereich des Postwesen der Deutschen Post, 1986, BStU, MfS, HA XIX, Nr. 7546, S. 1.

59 Vgl. Uli Schulte-Döinghaus: Eine »gesonderte Behandlung jenseits des Regellaufs«. Der Paketverkehr unter Kontrolle von Stasi und Zoll, in: Christian Härtel, Petra Kabus (Hrsg.): Das Westpaket: Geschenksendung, keine Handelsware, Berlin 2000, S. 65-80, hier S. 74. 
Der DDR selbst war es dabei besonders wichtig, medienwirksam gegen die Diebstähle vorzugehen. Einerseits, um den Bürgern zu versichern, dass ihre Eingaben ernstgenommen wurden, und andererseits, um die Öffentlichkeit davon zu überzeugen, dass es sich um Einzeltäter handelte und nicht um systematisch vorgenommene Raubzüge ${ }^{60}$ - eine Vermutung, die vor allem in der bundesrepublikanischen Presse immer wieder laut wurde. Diese Einzeltäter waren nach Überzeugung der Abteilung $M$ beim MfS vor allem unter jenen Postangehörigen zu finden, die »ungefestigte Einstellungen zum sozialistischen Eigentum haben ${ }^{61}$ und erst relativ kurz im Postdienst tätig waren wie zum Beispiel Aushilfs- oder Pauschalkräfte. Auf diese könne jedoch zu Zeiten der Verkehrsspitzen nicht verzichtet werden, da nur mit ihrer Hilfe die zeitlichen Vorgaben einzuhalten wären. ${ }^{62}$

Im Gegensatz zur ausgegebenen Täterbeschreibung - dies bestätigen die einzelnen Strafanzeigen und internen Auswertungen - waren es jedoch immer wieder auch langjährige Mitarbeiter der Post, die ihr Wissen um die internen Kontrollabläufe nutzen, um sich an Gegenständen aus Geschenksendungen oder Briefen zu bereichern. ${ }^{63}$ Die Analysen der Bezirksverwaltung und die internen Ermittlungen des MfS hingegen ergaben, dass sich die tatsächlichen Täterprofile und die Motive nicht immer mit den gesellschaftspolitischen Stereotypen, die in Bezug auf die Diebe ausgegeben wurden, in Einklang bringen ließen. So war die Mehrzahl der Diebe »im Arbeitskollektiv geachtet ${ }^{64}$, Mitglieder der SED und des FDGB, ${ }^{65}$ für ihre Arbeitsleistungen geschätzt und nicht selten sogar prämiert. ${ }^{66}$ Interessant ist, dass ein Großteil der Verurteilten angab, aus materiell und finanziell gesicherten Verhältnissen zu stammen, und dennoch ein Wunsch nach Westgütern und Westgeld bestand. ${ }^{67}$ Die erbeuteten Gegenstände wurden entweder zur eigenen Bedürfnisbefriedigung genutzt oder um einzelne Produkte einzutauschen, um andere Waren oder Dienstleistungen zu erhalten. Dass die meisten Beteiligten die Diebstählte nicht aus einer finanziellen Notlage heraus beging, sondern eher aus dem Wunsch nach west-

60 Vgl. Information zu Diebstählen von Post- und Paketsendungen in Berlin - Hauptstadt der DDR für den Zeitraum 01. Januar 1986 bis 30. Juni 1986, BStU, MfS, HA XIX, Nr. 7022, S. 2.

61 Zit. n. Ebd.

62 Vgl. Information zur Lage im Bereich des Postwesen der Deutschen Post, 1986, BStU, MfS, HA XIX, Nr. 7546, S. 6.

63 Vgl. Information zu Diebstählen von Post- und Paketsendungen in Berlin - Hauptstadt der DDR für den Zeitraum 01. Januar 1986 bis 30. Juni 1986, BStU, MfS, HA XIX, Nr. 7022, S. 2.

64 Zit. n. Information über die Aufklärung umfangreicher Diebstahlshandlungen von Paket- und Päckchensendungen, Schwerin 7. Mai 1987, BStU, MfS, BV, Swm, AKG, Nr. 7, S. 162.

65 Vgl. Schlussbericht über eine Verurteilung, 30. Juni 1988, Bundesbeauftragter für die Unterlagen des Staatssicherheitsdiensts BStU, MfS, HA IX, Nr. 4635, S. 9.

66 Vgl. Ebd., S. 10 und S. 12.

$67 \mathrm{Vgl}$. Ebd. 
lichen Produkten, liest sich wie eine Bankrotterklärung für die DDRBemühungen, durch die verschiedenen sozialen Sicherungssysteme und die Subventionen in vielen Bereichen des alltäglichen Lebens die Menschen vom eigenen System zu überzeugen.

\section{Die Ankunft dies- und jenseits der Grenze}

Auch der Empfang von Päckchen und Paketen gestaltete sich in West und Ost unterschiedlich. Im Osten Deutschlands war es vor allem die pure Freude über den Inhalt, der auf diesem Wege ins Haus kam. Obwohl die Westpakete trotz leichter Veränderungen stets zum Großteil aus Lebensmitteln bestanden - also Gegenständen, denen typischerweise nicht unmittelbar der Charakter eines Geschenkes beigemessen wird - war die Freude auf Seiten der Empfänger dennoch über die Maßen vorhanden. ${ }^{68}$ Diesen Lebensmitteln wurde schon allein deshalb ein besonderer Status zugeschrieben, da sie in der DDR kaum oder gar nicht zur Verfügung standen. Sie waren bereits durch ihre bunten und farbenfrohen Verpackungen, die es in der DDR so nicht gab, von einer Aura des Exquisiten umgeben. ${ }^{69} \mathrm{Oft}$ war allein das Auspacken ein Ereignis für die ganze Familie. Ihre Freude kommunizierten die Westpaketempfänger meist per Brief an ihre Bekannten und Verwandten im Absenderland. So kam es, dass schließlich beide Seiten den Lebensmitteln einen Geschenkcharakter zuschrieben. ${ }^{70}$

Die Ostpakete hingegen lösten nur selten ähnlich euphorische Gefühle aus, obwohl sie, abgesehen von den regionalen Spezialitäten, mit Büchern, Kalendern und kunstgewerblichen Gegenständen viel eher Dinge enthielten, denen ein Geschenkcharakter zugeschrieben werden kann. Genau dieser Umstand ließ die Freude auf Seiten der Ostpaketempfänger jedoch schwinden. Denn anders als die Lebensmittel, die Verbrauchsgegenstände waren, stellten die geschnitzten Schwippbögen ${ }^{71}$ und umhäkelten Deckchen Gebrauchsgegenstände dar, deren Anzahl sich durch die Benutzung nicht verringerte. Nicht jeder freute sich über den dritten oder vierten Schwippbogen - vor allem, wenn diese Art von kunstgewerblichen Geschenken so gar nicht den eigenen Geschmack traf. ${ }^{72}$ Darüber hinaus war im Westen die Freude über die Pakete teilweise gedämpft, weil die Menschen aus der Bundesrepublik davon über-

68 Vgl. Interview Frau Dilling am 17. Februar 2015.

69 Vgl. Interview Frau Winterfeld am 4. Februar 2015.

70 Vgl. Lindner: 2000, S. 26.

71 Vgl. Interview Frau Lindner am 26. Februar 2015.

72 Vgl. Interview Frau Walter am 28. November 2014. 
zeugt waren, dass es um ihre Landsleute im Osten schlechter bestellt sei. Sie sollten deshalb für sie nicht unnötig Geld ausgeben oder ihre Zeit mit Anstehen vergeuden. Dass für ihr Gegenüber der eigene Versand jedoch wichtig war, um nicht nur als Nehmende wahrgenommen zu werden, war vielen in der Bundesrepublik nicht bewusst. ${ }^{73}$

Doch es gab auch Freude bei den westdeutschen Empfängern. Besonders groß war sie, wenn regionale Spezialitäten enthalten waren. Denn all jene, die selber einmal in der DDR gelebt hatten, fühlten sich so ein bisschen in ihre ehemalige Heimat zurückversetzt. ${ }^{74}$

\section{Die Unterstützung der polnischen Bevölkerung}

Als die Bundesregierung Anfang der 1980er-Jahre dazu aufrief, Päckchen und Pakete nach Polen zu versenden, blickten die Deutschen dies und jenseits der innerdeutschen Grenze bereits auf eine lange Tradition des Geschenkpaketversandes zurück. Doch die Deutschen versandten bereits von Anfang an nicht nur Päckchen und Pakete in den jeweils anderen Teil Deutschlands, sondern auch nach Polen.

Früh gründete sich vor allem in der Bundesrepublik eine Vielzahl von Hilfsorganisationen, die sich im Versand von Päckchen und Paketen in die DDR engagierten. Ein Teil von ihnen - stellvertretend sei hier der Deutsche Frauenring mit dem ihm angeschlossenen Hilfsring zu nennen - versandte ebenfalls Päckchen und Pakete nach Polen. ${ }^{75}$ Denn durch die Kriegswirren hatten viele der Flüchtlinge, die aus dem heutigen Polen stammten, Kontakte zu Leuten, die einer Unterstützung bedurften. Die ehrenamtlich arbeitenden Frauen packten dabei nicht nur die Geschenksendungen, sondern organisierten Spenden und standen auch in brieflichem Kontakt mit den Empfängern in Polen. Eine Voraussetzung, um mit Päckchen und Paketen des Frauenrings bedacht zu werden, bestand darin, dass sich Empfänger und Versender nicht kennen durften. Sendungen an Bekannte und Verwandte sollten die Frauen ausschließlich aus eigenen finanziellen Mitteln tragen. Damit die Freude auf Seiten der Empfänger besonders groß war, waren die Frauen stets bemüht, die Geschenksendungen so individuell wie möglich zusammenzustellen. So vermerkten sich die Versenderinnen persönliche Eigenschaften und Merkmale, die im Laufe des Kontaktes ausgetauscht wurden, wie beispielsweise Geburts-

73 Vgl. Interview Herr Seifert am 7. Mai 2015.

74 Vgl. Interview Frau Winterfeld am 04. Februar 2015.

75 Vgl. Interview Frau Feldkamp am 3. Dezember 2014, 01:55:39. 
jahr, Kleidungsgröße oder Vorlieben gewissenhaft. ${ }^{76}$ Aus diesem Grund gelang es ihnen im Rahmen ihrer Möglichkeiten ganz besonders, mit den Geschenksendungen eine Freude zu bereiten.

Während der deutsch-deutsche Versand von Geschenksendungen in den 1970er-Jahren rückläufig war, stieg der nach Polen in den 1980er-Jahren an. ${ }^{77}$ Dieser Anstieg war dabei jedoch nicht auf den innerdeutschen Austausch von Päckchen und Paketen zurückzuführen, sondern auf die Sendungen, die weiter ostwärts nach Polen verschickt wurden.

Denn nachdem es in Polen Anfang der 1970er-Jahre, auch bedingt durch Kredite der Bundesrepublik, zu einem wirtschaftliche Aufschwung kam, verschlechterte sich die wirtschaftliche Lage Mitte der 1970er-Jahre wieder zusehends. ${ }^{78} \mathrm{Um}$ diese Situation zu entschärfen, entschloss sich die polnische Regierung, einen Teil der bis dahin existierenden Subventionen aufzuheben, mit der natürlichen Folge, dass sich nun viele Produkte des täglichen Bedarfs für die eigene Bevölkerung verteuerten. Weil es auch in den folgenden Jahren nur schwer gelang, die wirtschaftliche Situation im eigenen Land zu verbessern, sah sich die Regierung gezwungen, erneute Preiserhöhungen ab dem 1. Juli 1980 durchzusetzen. Da die polnische Regierung den Unmut der eigenen Bevölkerung fürchtete, wurden die neuen Maßnahmen erst einen Tag nach Inkrafttreten in der Presse veröffentlicht. Die sich immer weiter verschlechternde wirtschaftliche und soziale Lage führte zu Unruhen in weiten Teilen der Bevölkerung und löste ab August 1980 eine große Streikwelle aus. Ebenfalls 1980 gründete sich die Gewerkschaft Solidarność. Bis zum Sommer 1983 litt die polnische Bevölkerung unter einer so schlechten Versorgungslage, dass sich die Bundesregierung dazu entschied, die Menschen bei der Versorgung mit Lebensmitteln zu unterstützen. Durch die Paketaktionen - diese wurden auch von polenfreundlichen Kreisen der DDR mitgetragen - zielte die Bundesregierung darauf $\mathrm{ab}$, die entstandene Arbeiterbewegung, welche sich gegen das bestehende Regime stellte, zu unterstützen. ${ }^{79}$

So veranlasste die Bundesregierung für die Zeiträume vom 8. Februar 1982 bis 30. Juni 1982 sowie vom 1. November 1982 bis 31. Dezember 1982, dass die Versender von Geschenksendungen nach Polen die Beförderungsgebühren nicht selbst zahlen mussten, sondern die Pakete kostenlos aufgeben konnten. Bundesregierung und Bundesrat verfolgten damit das Ziel, der einer »akuten, weit über die sonstigen Versorgungsengpässe in Ostblockstaaten hinausgehen-

76 Vgl. Ebd., 01:09:08.

77 Vgl. Soch 2018, S. 241.

78 Vgl. Jens Ebert: Von der Feldpost zum zivil(isiert)en Briefverkehr.

79 Vgl. Ebd. 
den Notlage ausgesetzten polnischen Bevölkerung ${ }^{80}{ }^{\mathrm{zu}}$ helfen. Der Spiegel titelte am 7. Juni 1982 sogar »Polen-Hilfe: Eine echte Volksbewegung « ${ }^{81}$. Doch diese rosige Überschrift trog, wie auch die Leser des Artikels im weiteren Verlauf feststellen mussten, denn viele, die in diesem Zeitraum dem Aufruf der Bundesrepublik folgten und das polnische Volk unterstützen wollten, taten es vorrangig während der Zeiträume, in denen der Versand von der Bundesrepublik finanziert wurde und sahen es mehrheitlich als eine nur für kurze Zeit notwendige Unterstützung an ${ }^{82}$ - so lange, bis die schlimmsten Versorgungsengpässe vorüber waren. ${ }^{83}$ Diese Art der Hilfeleistung ließ sich nur in der Bundesrepublik finden, denn eine offizielle Unterstützung durch die DDR für die polnische Bevölkerung hätte zugleich eine Kritik am Bruderstaat bedeutet.

Die von der Bundesrepublik ins Leben gerufene humanitäre Hilfe spaltete die Päckchen- und Paketversender in zwei Lager, jedoch geeint in der Forderung, dass auch für Geschenksendungen in die DDR die Möglichkeit bestehen müsse, diese zu bestimmten Zeiten kostenlos zu versenden. Viele Versender wandten sich mit ihrem Unmut an das Bundesministerium für Post- und Fernmeldewesen sowie an das Bundespräsidialamt. Die Eingaben ähnelten sich in der Beschreibung der eigenen Lebenssituation und in der Einschätzung, in welchem Umfang der Versand von Geschenksendungen in die DDR die eigene finanzielle Situation belaste. Dies diente meist dazu, den Ausführungen mehr Nachdruck beziehungsweise eine höhere Glaubwürdigkeit zu verleihen. Die große Mehrzahl der Briefverfasser war dabei bereits verrentet und hatte wie Frau Ziegler "treu und brav all die Jahre hindurch Freunde und Verwandte in der DDR mit Päckchen u. Paketen, trotz schmalen Geldbeutels, versorgt « ${ }^{84}$. Mittlerweile seien die Portokosten jedoch so hoch, dass sie sich den Versand nicht mehr leisten könne und ihren Verwandten und Bekannten mit großem Schamgefühl mitgeteilt habe, dass sie den Versand einstellen müsse. Dies wiege ihrer Einschätzung nach besonders schwer, da die meisten Westpaketversender wie Frau Ziegler selbst aus der DDR stammen würden und sich aus diesem Grund verpflichtet fühlten, in Form von Geschenksendungen eine Unterstützung zu leisten. Des Öfteren verwiesen die Verfasser zudem darauf, dass bei einem Absenken der Portogebühren beziehungsweise einer zeitweise kostenlosen Beförderung der Sendungen das gesparte Geld nicht einbehalten, sondern

80 Zit. n. BArch Koblenz, B 257/21417, unpag.

81 Zit. n. Der Spiegel, 7. Juni 1982.

82 Vgl. Ebd.

83 Vgl. Brief an das Bundeministerium für Post- und Telekommunikation vom 12. Dezember 1982, BArch Koblenz, B 257/21417, unpag.

84 Zit. n. Brief an das Bundeministerium für Post- und Telekommunikation vom 12. November 1982, B 257/21417, unpag. 
ebenfalls wieder den Empfängern in der DDR zu Gute kommen würde. So könnten Pakete mit mehr Inhalt geschickt werden. ${ }^{85}$

Aus einem Teil der Briefe geht das konkrete Alter der Versender hervor. Viele sind in den 1920er- oder zu Beginn der 1930er-Jahre geboren, teilweise sind die Versender mit einem Geburtsjahr von 1917 sogar noch einige Jahre älter. Auffällig bei den Briefverfassern ist, dass sie ebenso wie Zeitzeugen dieser Jahrgänge immer wieder darauf verweisen, dass auch für sie der Versand von Geschenksendungen nicht immer einfach $\mathrm{zu}$ realisieren und mit finanziellen Beeinträchtigen verbunden sei.

Ein anderer Teil der Menschen begrüßte diese Unterstützung als ein »sehr löbliches Unternehmen ${ }^{86}$ und bejahte dessen Notwendigkeit. Dennoch sollten doch auch die Menschen in der DDR nicht vergessen werden, »denen es in diesem Jahr nicht viel besser geht als den poln. Menschen ${ }^{87}$. Um diesem Ansinnen Nachdruck zu verleihen, beschrieben die Verfasser immer wieder den Alltag in der DDR und wie dieser ihrer Meinung nach aussah - unabhängig davon, ob sie regelmäßig die DDR besuchten oder ob sie das letzte Mal vor mehr als zwanzig Jahren, als sie diese selbst verließen, vor Ort waren.

Ein weiterer Teil der Beschwerdeführer, wie beispielsweise Frau Fiedel, war erschrocken, »dass man so viel von Polen spricht und unsere eigenen Leute ${ }^{88}$ vergessen werden. Sie stellte im Anschluss die Frage:

»Wie komme ich dazu, für Polen portofrei Pakete zu schicken, wenn unsere Brüder diese selbst brauchen? Ist die Entfremdung schon so gross [sic], dass wir daran garnicht [sic] mehr denken??? ${ }^{89}$

Manche Versender, wie etwa Herr Kriebler, erachteten es sogar als vollkommen »unerhört « $^{90}$, dass Päckchen und Pakete portofrei nach Polen befördert würden. Schließlich sei es nicht das Verschulden der Deutschen, wenn »Polen nicht in der Lage ist, mit dem uns geraubten fruchtbaren Land seine Einwohner zu ernähren «" ${ }^{91}$. Nicht wenige Menschen stellten deshalb die Frage, ob »man unsere eigenen Landsleute in der DDR vergessen $«{ }^{92}$ würde. Dies wurde für sie zu einer Frage der Gerechtigkeit - schließlich gehe es den Menschen in

85 Vgl. Ebd.

86 Zit. n. Ebd.

87 Zit. n. Ebd.

88 Zit. n. Brief an das Bundeministerium für Post- und Telekommunikation vom 2. Dezember 1982, BArch Koblenz, Ebd.

89 Jens Ebert: Von der Feldpost zum zivil(isiert)en Briefverkehr.

90 Zit. n. Ebd.

91 Zit. n. Ebd.

92 Zit. n. Welt am Sonntag, 19. Dezember 1982. 
der DDR ebenso schlecht wie jenen in Polen. ${ }^{93}$ Mehr noch, was sollten die Menschen in der DDR davon halten, dass für die polnische Bevölkerung eine solche umfassende Unterstützung möglich sei, für die Menschen in der DDR jedoch nicht? Die polnische Hilfsaktion müsse deshalb laut Frau Fiedel im anderen Teil Deutschlands zwangsläufig zu der Meinung führen, dass man abgeschrieben worden sei, und als erneuter Beweis betrachtet werden, dass die Entfremdung zwischen beiden Teilen Deutschlands stetig zunehme. ${ }^{94}$ Zudem befürchtete Frau Ziegler, die Menschen in der DDR könnten dies als ein Zeichen deuten, dass die Polen der Bundesrepublik wichtiger seien als die eigenen Landsleute. ${ }^{95}$ Dies könne und dürfe jedoch nicht im Interesse der Bundesregierung sein. Immer wieder wurde in den Briefen auf die unermüdlichen Hinweise seitens der Politik verwiesen, die Kontakte in die DDR aufrechtzuerhalten. Den Paketversendern gelang es nur schwer, die offiziell vertretene Argumentation nachzuvollziehen, weshalb sie immer wieder die Frage stellten, »warum die Pakete nach dort [Polen] frei sind und $\mathrm{zu}$ unseren eigenen Landsleuten in die DDR nicht « ${ }^{96}$.

Doch nicht nur die Bundesregierung unterstützte in den 1980er-Jahren verstärkt den Versand von Polenpaketen, auch viele kirchliche Gemeinden engagierten sich in diesem Bereich. Die Zeitzeugin Frau Pirner erlebte Anfang der 1960er-Jahre, dass ihre Schulklasse in Konstanz darum gebeten wurde, Päckchen und Pakete in den Osten Deutschlands zu verschicken. Im Vergleich dazu beschrieb sie ihren eigenen späteren Versand von Polenpaketen in den 1980erJahren als unkomplizierter. Dies begründet sie für sich vor allem damit, dass es Anfang der 1960er-Jahre - als sie als Klassensprecherin dafür Sorge zu tragen hatte, dass auch alle Pakete befüllt wurden ${ }^{97}$ - für einen Teil der Mitschüler schwierig war, einen Beitrag zu leisten. Entweder, weil die Familien schon ihre Verwandten in der DDR unterstützten oder ihre finanzielle Lage es ihnen noch nicht ermöglichte, zusätzliche Ausgaben für den Inhalt von Geschenksendungen zu tätigen. ${ }^{98}$ In den 1980er-Jahren hingegen nahm sie das Verschicken der Polenpakete als wesentlich unkomplizierter wahr, da die Lebensmittel, die verschickt wurden, für jeden zumeist ohne großen Aufwand oder eine große finanzielle Belastung erworben werden konnten. ${ }^{99} \mathrm{Da}$ die Kirchgemeinde der

93 Vgl.: Brief an den Bundespräsidenten vom 30. Oktober 1983, BArch Koblenz, B 122/ 33650, unpag.

94 Vgl. Brief an das Bundeministerium für Post- und Telekommunikation vom 7. Dezember 1982, BArch Koblenz, B 257/ 21417, unpag.

95 Vgl. Ebd.

96 Zit. n. Ebd.

97 Vgl. Interview Frau Pirner am 10. Dezember 2014, 00:01:20.

98 Vgl. Ebd., 00:04:47.

99 Vgl. Ebd., 00:22:00. 
Zeitzeugin in Polen direkt eine Patengemeinde hatte, blieben die Kontakte länger bestehen als bei den Versandaufrufen durch die Bundesregierung. ${ }^{100}$

Ähnlich wie beim Versand der Päckchen und Pakete zwischen DDR und BRD zeigte sich auch beim Versand der Polenpakete, welche stereotypen Vorstellungen die Menschen über das Leben des jeweils anderen hatten. Einerseits waren viele Menschen dazu bereit, ihre polnischen Nachbarn zu unterstützen. Es erschien ihnen nur folgerichtig und konsequent. Denn schließlich »litten« die Menschen in der DDR wie in Polen ihrer Ansicht nach auch unter dem Einfluss der Sowjetunion. Ein solches Leben war für die Verfasser der Briefe zwangsläufig mit Problemen behaftet. ${ }^{101}$ Andererseits schwang bei dieser Hilfe auch eine nicht $\mathrm{zu}$ unterschätzende Ablehnung mit. In erster Linie jedoch keineswegs, weil die Versender in der Bundesrepublik nicht der polnischen Bevölkerung helfen wollten. Viel eher richtete sich die Unzufriedenheit der Menschen im Westen Deutschlands gegen die eigene Regierung. Denn diese betonte schon seit Jahren wie wichtig es sei, den Päckchen- und Paketverkehr aufrecht zu erhalten. Und obwohl die Möglichkeit bestand, einen Teil der Kosten für den Inhalt der Geschenksendungen steuerlich geltend zu machen, fühlten sich die Westpaketversender über die Jahre hinweg in ihren Bemühungen nicht ausreichend unterstützt. Dies wird an zahlreichen Eingaben der Versender deutlich. ${ }^{102}$ Besonders interessant erscheint die Befürchtung der Versender in der Bundesrepublik, dass sich die Menschen in der DDR durch den zeitweise kostenlosen Versand der Polenpakete zurückgesetzt beziehungsweise deklassiert vorkommen könnten. Bei den brieflichen Eingaben fällt auf, dass es letztlich nur Vermutungen und Befürchtungen der Menschen in der Bundesrepublik waren, wie der zeitweise kostenlose Versand auf die Menschen in der DDR wirken musste. Denn keiner der Menschen, die Eingaben verfasst hatten, gab zur Begründung ganz konkrete Äußerungen von Menschen aus der DDR oder aus Polen an.

\section{Der Päckchenversand nach der Wiedervereinigung}

Die Wiedervereinigung 1990 stellte die menschlichen Beziehungen zwischen Ost und West - ähnlich wie die voranschreitende Trennung unmittelbar nach Kriegsende - vor eine Herausforderung. Vieles änderte sich und bisher praktizierte Verhaltensweisen standen nun auf dem Prüfstand. Päckchen und Pakete

100 Vgl. Ebd., 01:10:31.

101 Vgl. Brief an den Bundespräsidenten vom 6. Juni 1983, BArch Koblenz, B 122/ 33650, unpag.

102 Vgl. Brief an das Bundeministerium für Post- und Telekommunikation von 16. Dezember 1982, BArch Koblenz, B 257/ 21417. 
waren nicht mehr nötig, denn der 'goldene Westen hielt Einzug in das eigene Leben östlich der Elbe.

Durch die Wiedervereinigung wurde in vielen Familien offenbar, dass den Päckchen und Paketen eine unterschiedliche Rolle zugewiesen wurde. So fasste ein Teil der westdeutschen Versender die Sendungen vor allem als milde Gaben auf, die keiner Erwiderung bedurften, da diese ohnehin nur bedingt möglich war beziehungsweise die Dinge, die sie zurückerhielten, nur in geringem Maße eine Freude auslösten. Aus diesem Grund wurde auf den Wunsch nach einer Gegengabe verzichtet.

Ein anderer Teil der westdeutschen Versender hingegen empfand Dank für die von ihnen versandten Gegenstände als wünschenswert, wenn auch nicht notwendig, da auf diesem Wege die Wertschätzung für die erhaltenen Geschenke zum Ausdruck gebracht wurde.

Nach der Wiedervereinigung schien dieser Dank in manchen Familien nach Einschätzung Einzelner jedoch nicht ausreichend artikuliert worden zu sein, sodass eine Art Aufrechnung entstand, in welcher dargelegt wurde, wer wie viel Zeit und Geld in den Versand investiert hatte. Neben dem Aspekt der gefühlten unzureichenden Dankbarkeit existierte in besonderer Weise die Herausforderung, das jahrelang praktizierte Rollenbild zwischen wohlhabendem Geber und bedürftigem Nehmer neu zu verhandeln - das führte auch auf westdeutscher Seite zu Enttäuschungen.

Der immer wieder sowohl von der Bundesrepublik als auch der DDR aufgestellten Behauptung, dass der Austausch von Geschenksendungen den Kontakt zwischen den Menschen in der Bundesrepublik und in der DDR sichern würde, lässt sich deshalb nur bedingt zustimmen. So trug er zwar dazu bei, Beziehungen zu pflegen, jedoch vor allem bei jenen, die sowieso z. B. familienbedingt Kontakte in den jeweils anderen Teil Deutschlands hatten. Die Dauer der Teilung trug zur Entfremdung bei, da durch den Wechsel der Generationen auf beiden Seiten Versender und Empfänger keinen Bezug mehr zueinander hatten oder verstarben.

Die registrierten Versandzahlen und die reine Aufrechterhaltung der Kontakte sagen zudem wenig über die eigentliche Intensität der Kommunikation aus. So wurde oft aus Gewohnheit am Versand festgehalten, vertieft hat er die persönlichen Kontakte aber nicht. Im vereinten Deutschland erhielt der Versand und Empfang sowohl im Osten als auch im Westen - trotz der Dankbarkeit und der Mühen, die er hervorgerufen hatte - zum Teil eine negative Konnotation. Dennoch - und dies muss mit allem Nachdruck festhalten werden blieben die Empfänger und Versender durch den Versand - auf welche Art, ist jeweils zu differenzieren - aufeinander bezogen. Und dies ist eine Funktion des Geschenkpaketversands, die nicht zu gering geschätzt werden kann, da beste- 
hende Kontakte ansonsten schon viel eher abgebrochen wären. Gleichzeitig wirken die Rollenzuschreibungen der vermeintlich armen Nehmenden im Osten und der vermeintlich reichen Gebenden im Westen in den Köpfen und Herzen der Menschen auch heute - 30 Jahre nach der Wiedervereinigung nach.

\section{Literaturverzeichnis}

Büro des Ministerrates der Deutschen Demokratischen Republik (Hrsg.): Gesetzesblatt der Deutschen Demokratischen Republik, Teil 1, 74/1954, Berlin 20.08.1954.

Uli Schulte-Döinghaus: Eine "gesonderte Behandlung jenseits des Regellaufs«. Der Paketverkehr unter Kontrolle von Stasi und Zoll, in: Christian Härtel, Petra Kabus (Hrsg.): Das Westpaket: Geschenksendung, keine Handelsware, Berlin 2000, S. 65-80.

Jens Ebert: Von der Feldpost zum zivil(isiert)en Briefverkehr, in: www.museums-stiftung.de /briefsammlung/post-von-drueben/briefsammlung-ziviler-briefverkehr.html (letzter Zugriff: 28.11.2015).

Josef Foschepoth: Überwachtes Deutschland. Post- und Telefonüberwachung in der alten Bundesrepublik, Göttingen 2013.

Jörn-Michael Goll: Innenansichten. Die Kontrolle des deutsch-deutschen Paket- und Päckchenverkehrs durch Zoll und Staatssicherheit in der DDR, in: DA, 40 (2007) 6.

Rainer Gries: »Jacobs Kaffee - wunderbar« - Päckchen nach drüben: Eine geniale Verbindung von Produktwerbung und Politikpropaganda, in: Blum, Mechthild Blum und Thomas Nesseler (Hrsg.): Deutschland, einig Vaterland? Geschichte(n), Probleme und Perspektiven, Bonn 1992, S. 80-85.

Christian Härtel: Ostdeutsche Bestimmungen für den Paketverkehr im Spiegel westdeutscher Merkblätter, in: Christian Härtel, Petra Kabus (Hrsg.): Das Westpaket: Geschenksendung, keine Handelsware, Berlin 2000, S. 45-56.

Hilfsring e.V. 1953-1973, Broschüre des Frankfurter Hilfsrings, Frankfurt a. M. 1973.

Bernd Lindner: "Dein Päckchen nach drüben«. Der deutsch-deutsche Paketversand und seine Rahmenbedingungen, in: Christian Härtel, Petra Kabus (Hrsg.): Das Westpaket: Geschenksendung, keine Handelsware, Berlin 2000, S. 25-44.

Monika Sigmund: Genuss als Politikum. Kaffeekonsum in beiden deutschen Staaten (Studien zur Zeitgeschichte Bd. 87), Berlin/München/Boston 2015, S. 202.

Konstanze Soch: Eine große Freude? Der innerdeutsche Paketverkehr im Kalten Krieg (1949-1989), Frankfurt am Main/New York 2018. 
Open-Access-Publikation im Sinne der CC-Lizenz BY-NC-ND 4.0 (C) 2021, Vandenhoeck \& Ruprecht GmbH \& Co. KG, Göttingen ISBN Print: 9783847112280 - ISBN E-Lib: 9783737012287 


\section{Abbildungen}

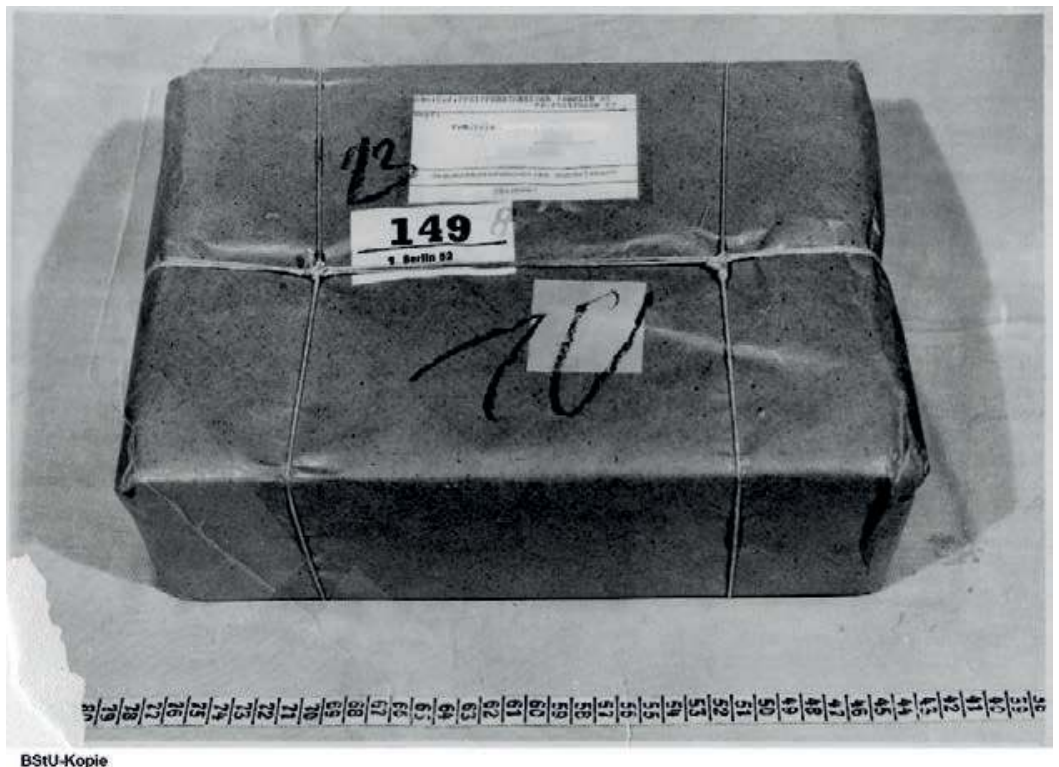

Abbildung 1: Kontrolliertes Westpaket versehen mit dem Hinweis "Geschenksendung - keine Handelsware«, ohne Datum.

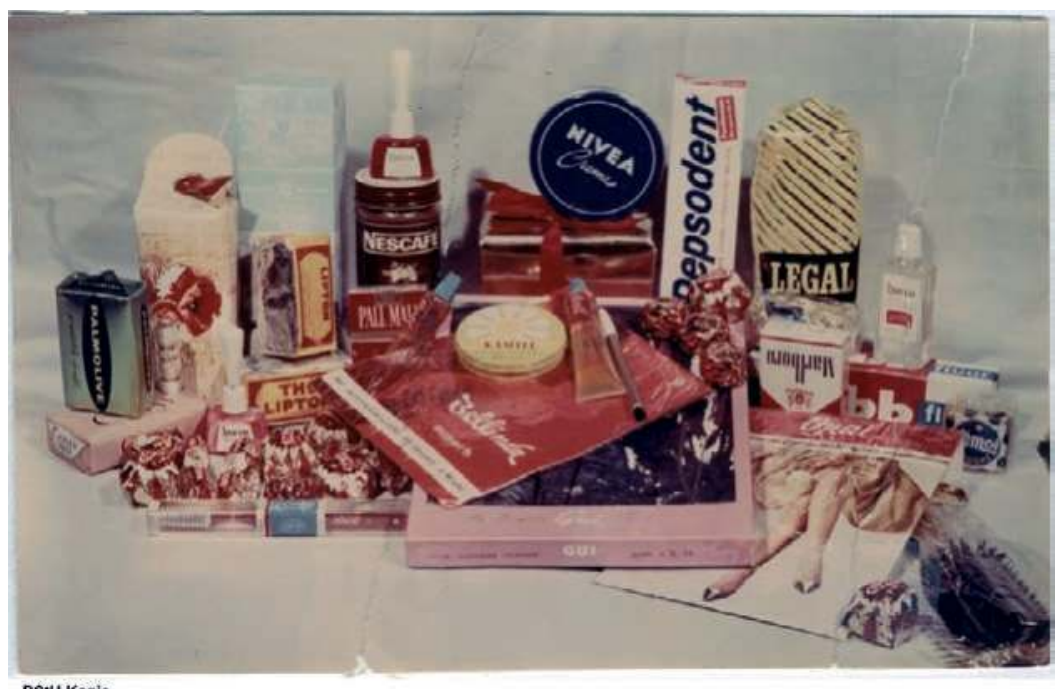

BStU-Kopie

Abbildung 2: Inhalt eines kontrollierten Westpakets, ohne Datum. 


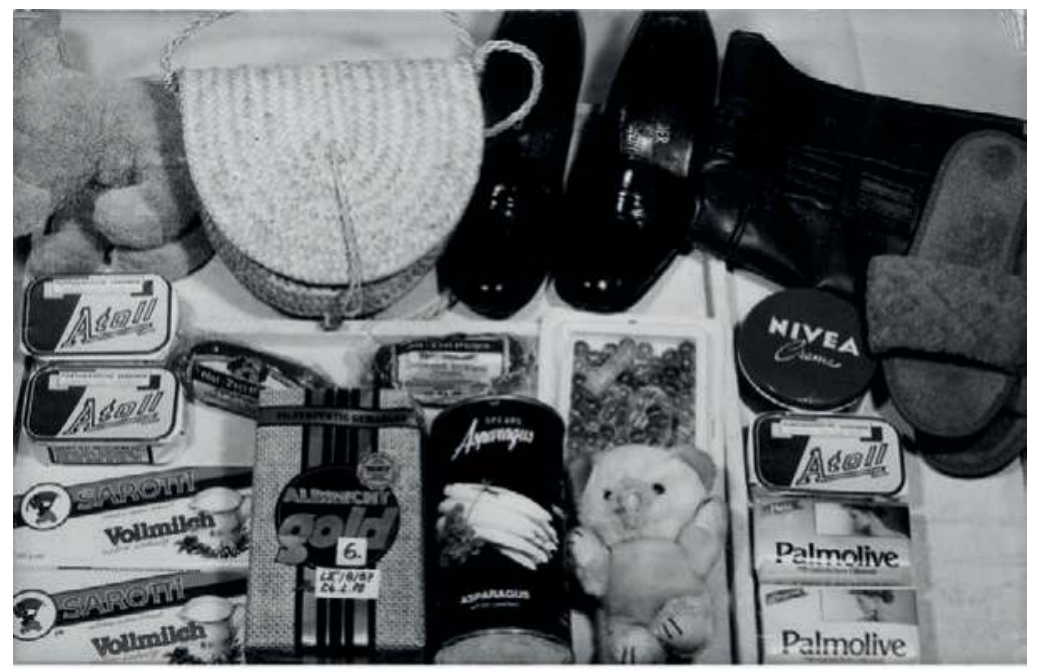

BstU-Kopie

Abbildung 3: Kontrolliertes Westpaket für eine Familie in Leipzig, ohne Datum.

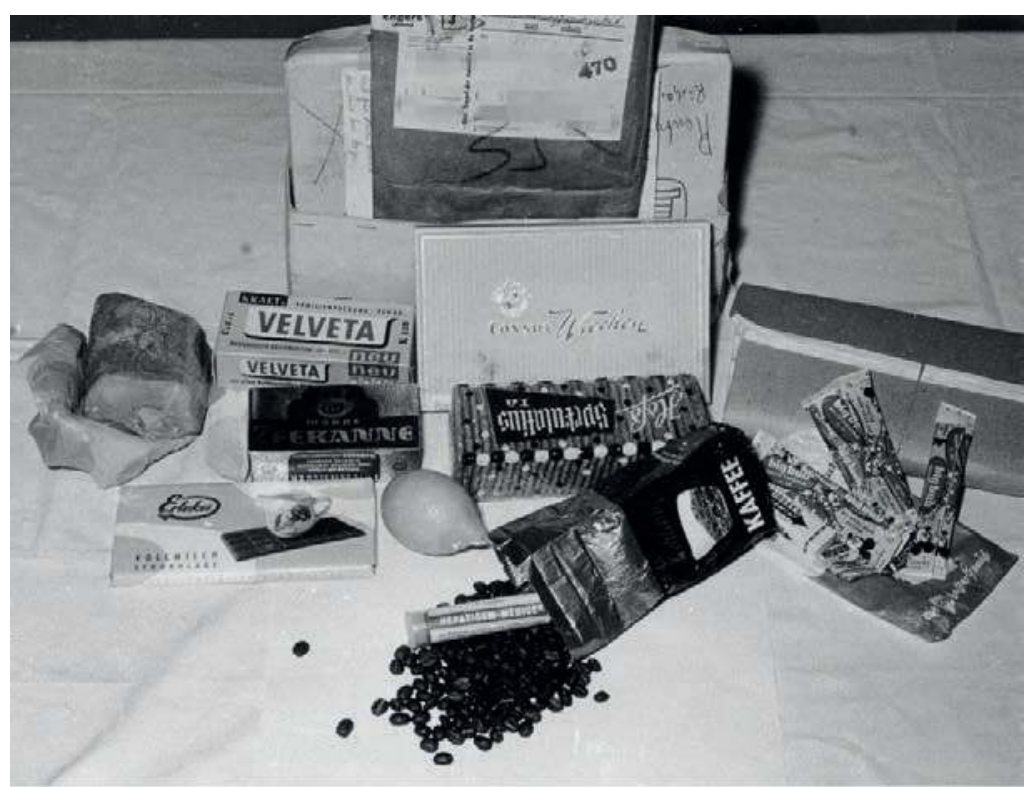

BStU-Kopie

Abbildung 4: Kontrolliertes Westpaket, in welchem in einer Kaffeeverpackung Medikamente versteckt waren, ohne Datum. 


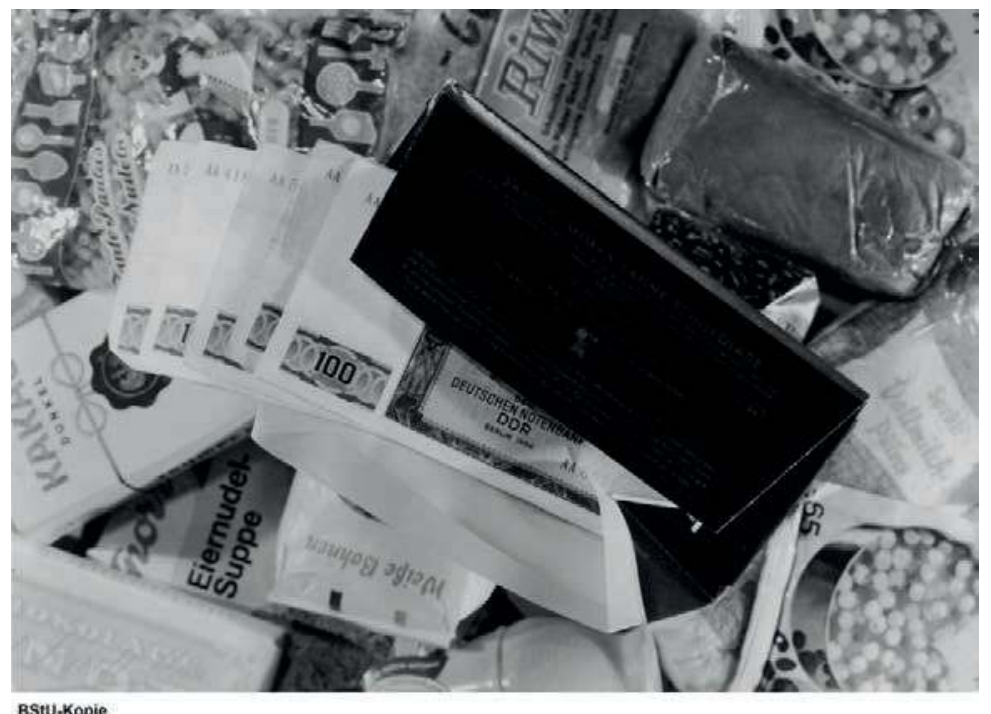

Abbildung 5: Von der Stasi geöffnete Schokoladenverpackung, in welcher DDR-Mark versteckt waren, ohne Datum. 
Open-Access-Publikation im Sinne der CC-Lizenz BY-NC-ND 4.0 (C) 2021, Vandenhoeck \& Ruprecht GmbH \& Co. KG, Göttingen ISBN Print: 9783847112280 - ISBN E-Lib: 9783737012287 


\section{Amelie Dreecke}

\section{Der Adel und die Ansichtskarte. Motivik im Dienste der Herrscher}

\section{Einleitung}

Über Jahrhunderte hinweg hatte das sogenannte einfache Volk nur äußerst limitierte Möglichkeiten einen näheren und tatsächlichen Blick auf ihre Herrschaften zu erhaschen. Alternativen boten dagegen diverse Abbildungsmechanismen. Münzprägungen porträtierten die Herrscher in einem, von antiken Traditionen idealisierten, Bild. ${ }^{1}$ Drucke, wie beispielsweise Kupferstiche, waren häufig ebenso idealisiert. ${ }^{2}$ Beeindruckende gemalte Portraits wurden meist in persönlichen Räumlichkeiten ${ }^{3}$ oder auch an offiziellen Orten aufbewahrt, zu denen meist nur die höheren Schichten der Bevölkerung Zugang hatten. ${ }^{4} \mathrm{~Pa}-$ raden oder Umzüge wurden von neugierigen und zugleich sichtversperrenden Menschenmassen besucht und waren zudem äußerst schnell vorüber. Folglich war das Vorhandensein eines tatsächlichen Bildes der Herrschenden, in all ihrer vorhandenen Varietät, dennoch begrenzt.

1 Als Einführungsliteratur sei hier Reinhard Wolters Beitrag erwähnt, vgl. Reinhard Wolters: Die Geschwindigkeit der Zeit und die Gefahr der Bilder: Münzbilder und Münzpropaganda in der römischen Kaiserzeit, in: Gregor Weber, Martin Zimmermann (Hrsg.): Propaganda - Selbstdarstellung - Repräsentation im römischen Kaiserreich des 1. Jhs. n. Chr., Wiesbaden 2003, S. 175-204. Für den weiteren historischen Verlauf siehe beispielsweise William McAllister Johnson: Numismatic propaganda in Renaissance France, in: Detroit Institute of Arts (Hrsg.): The art quarterly, 31, Detroit 1968, S. 123-153 oder Charles M. Rosenberg, Money talks. Numismatic propaganda under Alfonso I d'Este, in: Angela Ghinato (Hrsg.): L' età di Alfonso I e la pittura del Dosso, Modena 2004, S. 145-164.

2 Selbstredend konnten besonders Druckwerke von politischen Widersachern oder Andersdenkenden karikativ genutzt werden und so den Herrscher in eine negative Art porträtieren.

3 Als prominentes Beispiel sei Kaiser Franz Joseph I. (1830-1916) genannt, der in seinem Arbeitszimmer im Wiener Schloss Schönbrunn diverse Portraits seiner Gattin Kaiserin Elisabeth (1837-1898) angebracht hatte, unter anderem jene Gemälde von Franz Xaver Winterhalter (1805-1873), die die Kaiserin mit offenen Haaren zeigen und nur für die Augen des Kaisers geschaffen worden waren.

4 Vgl. Lutz Hieber: Gesellschaftsepochen und ihre Kunstwelten, Wiesbaden 2018, S. 2. 
Dank der Erfindung der Daguerreotypie und ihrer Weiterentwicklung zur Fotografie veränderte sich der Zugang zu visuellen Materialien enorm. Bei der Carte de visite, 1854 von André Adolphe Eugène Disdéri (1819-1889) patentiert, und der Kabinettkarte handelte es sich um kleinformatige Fotografien, die sich in der zweiten Hälfte des 19. Jahrhunderts einer großen Beliebtheit und Verbreitung erfreuten und zu begehrten Sammelobjekten avancierten. ${ }^{5}$ Nicht nur Fotografien, die einen selbst, Familienmitglieder oder Freunde zeigten wurden gesammelt, sondern ebenso Aufnahmen, die berühmte Zeitgenossen abbildeten, wie Schriftsteller, Schauspieler oder eben auch Adelige. ${ }^{6}$

Solche Fotografien wurden häufig zur Bebilderung von Ansichtskarten genutzt. Karten mit einer Abbildung auf der Rückseite wurden in der zweiten Hälfte des 19. Jahrhunderts ein alltägliches Kommunikationsmittel. In diesem Beitrag liegt das besondere Augenmerk auf den Ansichtskarten, die den Adel darstellen. Auf welche Art und Weise ließ sich der Adel auf Ansichtskarten darstellen? Welche Wege der Propaganda wurden für seine diversen Zwecke, beispielsweise die moralische und finanzielle Unterstützung von Kriegen oder wohltätiger Vereine, gewählt? Lassen sich stilistische Unterschiede zu anderen Darstellungsmöglichkeiten, wie dem klassischen, gemalten Portrait, ausmachen, oder ist die Ansichtskarte ein schlichtes Objekt, das vollends im Dienste der Herrschermotivik stand und kaum Raum für Gegenbewegungen, wie Kritik aus den Reihen der Bevölkerung, bot? Dies sind nur einige der Fragen, die in diesem Beitrag behandelt werden sollen. Um sich der Beantwortung dieser Fragen anzunähern, wurden Ansichtskarten vor allem aus dem Zeitraum von um 1900 bis um 1914 ausgewählt, einer Zeit, in der das Medium der Ansichtskarte in einem hohen Maße vertrieben wurde. Der Auswahl der Karten liegt eine Beschränkung auf Zentraleuropa zugrunde, wobei das Hauptaugenmerk auf dem Deutschen Kaiserreich liegt. Die in diesem Beitrag besprochenen Ansichtskarten wurden nach dem geographisch-zeitlichen Rahmen, ästhetischen Belangen und der Veranschaulichung der thematischen Diversität ausgewählt.

Kommunikation, nonverbal und verbal, ist ein äußerst breiter Forschungsbereich, der Wissenschaftler und Forscher über Jahrhunderte und Kulturen hinweg fasziniert. ${ }^{7}$ Ansichtskarten, obwohl sie Kunst, Kultur und nonverbale

5 Vgl. Ulla Fischer-Westhauser, Gerda Mraz: Elisabeth. Prinzessin in Bayern, Kaiserin von Österreich, Königin von Ungarn. Wunschbilder oder die Kunst der Retouche, Wien 1998, S. 37.

6 So existieren beispielsweise Karten, die Charles Dickens (1812-1870), Queen Victoria (18191901) oder die Schauspielerin Sarah Bernhardt (1844-1923) zeigen.

7 Als Beispiele seien an dieser Stelle folgende Forschungsarbeiten erwähnt: Vgl. Karen A. Foss, Stephen W. Littlejohn, John G. Oetzel: Theories of human communication, Long Grove 2017, Siegfried Frey: Die Macht des Bildes. Der Einfluß der nonverbalen Kommunikation auf Kultur und Politik, Bern 1999 oder ebenso Walter Goldschmidt: Nonverbal Communication and Cul- 
Kommunikation in sich vereinen, waren bisher allerdings noch kein Teil eines größeren Forschungsfeldes. An dieser Stelle sei auf das wahrscheinlich umfangreichste Werk verwiesen, das zu Ansichtskarten veröffentlicht wurde. Otto May veröffentlichte erstmals 1998 seine Forschungsergebnisse über die Untertanerziehung durch Ansichtskarten im deutschen Kaiserreich. ${ }^{8}$ Für seine Untersuchungen zog May 13000 Ansichtskarten heran, was seine Ergebnisse auf eine aussagekräftige Basis stellt. Seine Monographie ist in drei thematische Hauptglieder unterteilt, ${ }^{9}$ die jeweils in verschiedene Unterthemen unterteilt sind. Diese enorme thematische Fülle ist bedauerlicherweise auch ein negativer Aspekt in Mays Werk. Auf rund 700 Seiten behandelt May diverse Themenaspekte, doch ist so kaum Raum für tiefere Forschung, mehr ist es ein Anreißen der Felder. So beschäftigt er sich beispielsweise mit den Nationalsymbolen und Nationalfiguren des Rheins, des Brandenburger Tors und dem »Problem der Nationalhymne« auf lediglich einer einzigen Seite. ${ }^{10}$ Diese Verknappung verweist indirekt sehr gut auf die Komplexität des Themenfeldes und ist für die wissenschaftliche Beschäftigung mit Ansichtskarten während der Kaiserzeit in jedem Fall ein guter Startpunkt und eine unerlässliche Stütze. ${ }^{11}$ Ebenso umfangreich ist die Arbeit von Robert Lebeck und Gerhard Kaufmann, die sich bereits 1985 mit einer Kulturgeschichte der Postkarte befassten. ${ }^{12}$ Besonders die historischen Gegebenheiten sind sehr detailliert und aufschlussreich dargestellt. Zudem beschäftigten sie sich auch mit der Ästhetik ${ }^{13}$ und den Gestaltern ${ }^{14}$ der Postkarten. Ein bedauerliches Versäumnis sind jedoch die fehlenden Literatur- und Quellenverweise in ihrer Arbeit. Auch wenn die Autoren durchaus eine ausführliche Literaturauswahl anführen, so bleiben ihre spezifi-

ture, in: Ullica Segerstråle, Peter Molnár (Hrsg.): Nonverbal communication. Where nature meets culture, Mahwah 1997), S. 229-244.

8 Vgl. Otto May: Deutsch sein heißt treu sein. Ansichtskarten als Spiegel von Mentalität und Untertanerziehung in der Wilhelminischen Ära (1888-1918), Hildesheim 1998.

9 So der erste Teil »Untertanerziehung und das Medium der Ansichtskarte in der Ära des Wilhelminismus« (S. 11-96), der zweite Teil »Mentalitätspostkarten als Spiegel gesellschaftlicher Phänomene« (S. 97-653), der dritte Teil »Das konstruierte Modell der Untertanerziehung im Spiegel der Mentalitätspostkarten « (S. 654-685).

10 Alle Themenfelder in May 1998, S. 488.

11 Auch für die Zeit nach dem Kaiserreich liefert May umfangreiche Arbeiten, Otto May: Vom Wachsen lassen zum Führen. Die Ansichtskarte als Zeuge einer versäumten Erziehung zur Demokratie in der Weimarer Republik, Hildesheim 2003 sowie chronologisch folgend Otto May: Inszenierung der Verführung. Die Ansichtskarte als Zeuge einer autoritären Erziehung im III. Reich, Hildesheim 2003.

12 Vgl. Robert Lebeck, Gerhard Kaufmann: Viele Grüße... Eine Kulturgeschichte der Postkarte, Dortmund 1985.

13 Vgl. Ebd., S. 419-423.

14 Vgl. Ebd., S. 423-425. 
schen Äußerungen, speziell zum historischen Kontext, dadurch kaum nachzuvollziehen oder gar adäquat überprüfbar.

Ebenso umfangreich ist auch die Arbeit von Günter Formery und Thomas Fürst, die 2011 Die Welt des Ansichtskartensammelns herausgebracht haben. ${ }^{15}$ Einen größeren Raum finden in Formerys und Fürsts Arbeit die diversen Druckverfahren, die zur Gestaltung der Ansichtskarten genutzt wurden. ${ }^{16}$ Der Großteil der Publikation befasst sich allerdings mit dem Sammeln der Ansichtskarten und mit der Ermittlung der verschiedenen Preiskategorien, Vereine oder auch Börsen und ist von daher nur für bestimmte Fragestellungen von einem weiterführenden (wissenschaftlichen) Interesse. Nichtsdestotrotz zeigt die Arbeit eindrücklich, welche Bedeutung der Ansichtskarte als Sammelobjekt zukommt.

\section{Historie und Möglichkeiten der Ansichtskarte}

Karten mit geschriebenen Nachrichten, wie Grüße an die Daheimgebliebenen, Geburtstagsglückwünsche oder ähnliches, zu verschicken, ist für den Menschen des 21. Jahrhunderts keine Besonderheit. Der Sinn für Kommunikation und Visualisierung hat sich enorm verändert, vor allem in der letzten Dekade mit der Etablierung des Smartphones.

Die Idee eine Nachricht auf ein Stück Papier zu schreiben, die von jedem gelesen werden könnte, der das Papier in der Hand hält, war in diesem Sinne eine Neuheit des 19. Jahrhunderts. Über Jahrhunderte wurden Nachrichten über Boten verschickt, deren Ruf vor allem von ihrer Diskretion abhängig war. Nachrichten, offizielle wie private, waren im Grunde durch Umschläge versteckt und somit nicht sichtbar für das öffentliche Auge.

Der Anstieg der Alphabetisierung im Laufe der Jahrhunderte führte zu einem Anstieg an geschriebenen Briefen und Nachrichten. Zunehmend wurden Postunternehmen gegründet und ausgebaut, die versuchten durch ihre Schnelligkeit neue Kunden von sich zu überzeugen. ${ }^{17}$ Nichtsdestotrotz, als Heinrich

15 Vgl. Günter Formery, Thomas Fürst: Die Welt des Ansichtskartensammelns, Schwalmtal 2011. 16 Vgl. Ebd., S. 43-60.

17 Dank der Schnellpost, die sich in den 1820er Jahren in den deutschsprachigen Gebieten etablierte, konnten diverse Routen deutlich schneller mit Post versorgt werden. Die Attraktivität der Schnellpost stieg auf Grund der Möglichkeit der zum Teil stark verkürzten Transportzeiten. Auf manchen Strecken konnten die Zeiten gar halbiert werden, wie beispielsweise die Strecke zwischen Frankfurt am Main und Stuttgart, die ursprünglich 40 Stunden benötigte, die Schnellpost die jeweilige Destination jedoch bereits nach 24 Stunden erreichte. Vgl. Christine Haug: Reisen und Lesen im Zeitalter der Industrialisierung. Die Geschichte des Bahnhofs- und Verkehrsbuchhandels in Deutschland von seinen Anfängen um 1850 bis zum Ende der Weimarer 
von Stephan (1831-1897), der ab 1870 Generalpostdirektor zunächst des Norddeutschen Bundes und später auch des Deutschen Reichs wurde, im Jahr 1865 über die Einführung eines offen lesbaren Briefs sprach, einer Postkarte, blieb die Unterstützung seiner Idee vorerst aus. ${ }^{18}$ Dank der sogenannten Korrespondenzkarte, die 1869 durch die österreichische Postadministration und in Reaktion auf einen wissenschaftlichen Artikel des Ökonomen Emanuel Hermann (1839-1902) eingeführt worden war, war die erste Postkarte im eigentlichen Sinne geschaffen. ${ }^{19}$

Die damit verbundene Art der Kommunikation war ein derartiger Erfolg, dass Heinrich von Stephan es bei seinem Amtsantritt als Generalpostdirektor 1870 deutlich einfacher hatte, seine bereits 1865 geäußerte Idee eines offen lesbaren Briefes auch in Deutschland einzuführen.

Als der Vertrieb von Korrespondenzkarten am 25. Juni 1870 in Berlin begann, wurden bereits an diesem ersten Tag 45468 Karten verkauft, ${ }^{20}$ was für das enorme Interesse der Bevölkerung spricht.

Korrespondenzkarten waren streng unterteilt, eine Seite lediglich für die Adresse, die andere für die Nachricht, eben jene Korrespondenz, der das Objekt seinen Namen verdankte. Eine größere Beliebtheit der Korrespondenzkarte wurde erreicht, als mit der Zeit auch Bebilderungen auf den Karten abgedruckt wurden. Die offizielle Lizenz zum Druck solcher Ansichtskarten wurde 1885 in Deutschland beschlossen. Mit dieser Lizenz war es nicht nur der Reichsdruckerei erlaubt solche Ansichtskarten legal zu drucken, sondern auch private Initiativen und Unternehmen konnten seitdem ebenso Karten in den Druck bringen, so lange sie die offiziellen Vorgaben, wie das exakte Format oder eine gewisse Stabilität der Ansichtskarte, einhielten. ${ }^{21}$

Zur Illustration und Bebilderung der Ansichtskarten wurde jegliches Thema aufgegriffen, das einen kommerziellen Erfolg versprach. Der Themenspanne war folglich kaum eine Grenze zu setzen und reichte von Geburtstagswünschen, Momentaufnahmen prominenter Veranstaltungen wie dem Oktoberfest in München, Aufnahmen bestimmter Menschentypen, Karikaturen oder ähnlichem, wobei Fotografien, kopierte Gemälde, Kupferstiche, Zeichnungen und Weiteres Verwendung fanden. ${ }^{22}$

Republik, Wiesbaden 2007, S. 25-26. Zur Postgeschichte und ihrer Preisentwicklung siehe Johannes Rüberg: Die Preisbestimmung in der Telekommunikation, Tübingen 2017, S. 36-39.

18 Vgl. Lebeck, Kaufmann 1985, S. 401.

19 Vgl. Formery, Fürst 2011, S. 13.

20 Vgl. Lebeck, Kaufmann 1985, S. 402.

21 Vgl. Karin Walter: Die Ansichtskarte als visuelles Massenmedium, in: Wolfgang Kaschuba, Kaspar Maase (Hrsg.): Schund und Schönheit. Populäre Kultur um 1900, Köln 2001, S. 48.

22 Für die unterschiedlichen Möglichkeiten der Druckvorgänge und Drucktypen, die für das Herstellen von Ansichtskarten genutzt wurden siehe Formery, Fürst 2011, S. 43-60. 
Ansichtskarten avancierten zu einem Massenmedium, besonders vorangetrieben auf Grund des geringen Einkaufspreises. Um 1900 lag der Preis einer Postkarte in Deutschland bei rund 3 Pfennig, ohne Portokosten. Um einen Vergleich zu geben, sei darauf verwiesen, dass ein Kilogramm Brot im gleichen Zeitraum für 24 Pfennig zu erwerben war. ${ }^{23}$ Der vergleichsweise niedrige Preis unterstützte die Popularität der Postkarte, deren Beliebtheit sich bereits seit der zweiten Hälfte des 19. Jahrhunderts in einer stetig steigenden Anzahl von Kartensendungen widerspiegelte. Innerhalb von nur 15 Jahren erweiterte sich der Transport von Postkarten innerhalb Deutschlands enorm. Wurden im Jahr 1875 noch 61,9 Millionen Postkarten versendet, so waren es 1890 bereits 330,3 Millionen. ${ }^{24}$ Kaum messbar ist dagegen die Anzahl der Ansichtskarten, die nicht verschickt wurden, sondern als Erinnerungsstück behalten oder möglicherweise verschenkt wurden. Dass solche Karten ausgiebig gesammelt wurden, ist durch die Existenz von Sammelalben belegt, die vergleichbar sind mit Carte-de-Visite-Alben oder auch den späteren Fotoalben des 20. Jahrhunderts. Das Sammeln druckgraphischer Objekte kann dabei auf eine jahrhundertealte Tradition verweisen. Begründet wurde diese nicht zuletzt durch die Flugblätter in der Frühen Neuzeit, welche »neben tragischen Liebesgeschichten, Naturkatastrophen und anderen Unglücksfällen ebenfalls das Genre der Kriminalverbrechen $\aleph^{25}$ veranschaulichten. Die Nachfrage solcher Flugblätter war hoch, ${ }^{26}$ wobei eine besondere Nachfrage im Bereich der Kriminalitätsabbildungen vorherrschte, eine Vorliebe, die sich auch noch im 19. Jahrhundert immer wieder findet. ${ }^{27}$ Besonders im Falle der Verbrechens- oder Vollzugsdarstellungen wurde in der Metaebene die Abwesenheit und Anwesenheit der Macht des Adels dargestellt - eine dysfunktionale oder funktionale Justiz, die unter dem Herrscher ausgeführt wurde. Solche Graphiken vereinten offensichtliche bildliche sowie schriftliche Elemente, als auch in der Metaebene teils verschlüsselte Züge.

In diese Tradition kann sich auch die Ansichtskarte einreihen - bildliche und schriftliche Elemente jeglicher Ausrichtung konnten zu einem geringen Preis erworben werden, wodurch sie folglich von viele Menschen erworben,

\footnotetext{
23 Vgl. May 1998, S. 59.

24 Vgl. Walter 2001, S. 47.

25 Jutta Nowosadtko: Hinrichtungsrituale. Funktion und Logik öffentlicher Exekutionen in der Frühen Neuzeit, in: Michael Matheus, Sigrid Schmitt (Hrsg.): Kriminalität und Gesellschaft in Spätmittelalter und Neuzeit, Stuttgart 2005, S. 72.

26 Ebd.

27 Vgl. Uli Reiter: Illegalität. Phänomen und Funktion, Wiesbaden 2016, S. 211. Siehe zur historischen Druckgraphik im Bereich der Kriminalität auch Samuel Edgerton: Pictures and Punishment. art and criminal prosecution during the Florentine Renaissance, Ithaca 1985 oder Richard Ward: Print Culture, Crime and Justice in Eighteenth-Century London, London 2014.
} 
gesammelt oder verteilt werden konnten. Die Ansichtskarte ist ein wichtiges Bindeglied im Verlauf der nonverbalen, schriftlichen und bildlichen Kommunikationsentwicklung, in deren weiteren Verlauf auch die elektronischen Kurznachrichten angesiedelt werden können. Wie bereits angerissen, spiegelten sich in der Metaebene der frühneuzeitlichen und späteren Flugblätter und Druckgraphiken in der Regel gesellschaftliche Regelungen, Normen und Herrschaftsansprüche wider.

Der Adel gehörte damit von Beginn an zu einem wesentlichen Bestandteil der möglichen Bebilderung von Ansichtskarten, sowohl in der direkten Ansicht, als auch in der Metaebene.

In Bezug auf die Auseinandersetzung mit diesem Medium wird seine Beziehung zum Adel und dessen Darstellungsweisen in den Mittelpunkt gerückt und sind drei wesentliche Leitfragen zu nennen, die im Folgenden den weiteren Beitrag begleiten und die Beispielsauswahl bestimmen.

So soll mit diesem Beitrag untersucht werden, in welcher Art und Weise der Adel auf Ansichtskarten präsentiert wurde und welche Art von zeitgenössischer Propaganda und Wertvorstellungen durch die Präsentationen angesprochen und visualisiert worden sind. Die Propaganda und die Wertevorstellungen führen somit unweigerlich zum Ansehen, dem Image der abgebildeten Personen, welches durch die Ansichtskarten durch das gesamte Land, und über dessen geografischen Grenzen hinaus, getragen wurde.

Ebenso ist von Interesse, ob sich stilistische Unterschiede zwischen Ansichtskarten und klassischen Kunstwerken, wie der Malerei, erkennen lassen.

Um diesen Forschungsfragen zu folgen, wird der gängige Werdegang ${ }^{28}$ eines Adeligen um das Jahr 1900 durch diverse Ansichtskarten illustriert und untersucht, wobei es an geeigneten Stellen sowohl historische Vor- als auch Rückgriffe geben wird, um ein zusätzliches Verständnis für die Gestaltungen von Ansichtskarten zu gewährleisten.

Je nachdem, ob es sich um einen weiblichen oder männlichen Vertreter eines Adelsgeschlechts handelte, wurde dieser entsprechend ihrer oder seiner jeweiligen repräsentativen oder geschlechtsspezifischen Aufgaben dargestellt.

Die Gewährleistung des Fortbestands des Geschlechts und damit eine Sicherung von Kontinuität war seit jeher wesentlicher Bestandteil der Aufgabe weiblicher Adeliger. ${ }^{29}$ Mit der Bekanntgabe einer Verlobung und einer schließlich

28 Mit einem gängigen Werdegang sind die wesentlichen Lebensetappen gemeint, sprich Heirat, Kinder und Tod, also ein Kreislauf.

$29 »[\ldots]$ Für diejenigen Adelsfamilien, deren Strategie auf Ahnenproben und lupenreine Ahnenketten angewiesen war, verbot sich die bürgerliche Heirat von Söhnen und Töchtern ohnehin. [...] Die große Zahl von unverheiratet bleibenden Kindern, gerade auch der Töchter, zeigt an, dass Statuswahrung das primäre Ziel des Adels war.« Heinz Reif: Adel im 19. und 20. Jahrhundert, München 2012, S. 37. 
stattfinden Hochzeit ruhten und lasteten Erwartungen und Sorgen auf dem Ehepaar.

Die Gestaltungsweisen von Ansichtskarten in diesem thematischen Bereich konnten recht unterschiedlich ausfallen, was die folgenden Beispiele exemplarisch darstellen sollen.

Eine Postkarte anlässlich der Vermählungsfeier des Kronprinz Wilhelm von Preußen (1882-1951) mit Cecilie von Mecklenburg (1886-1954) im Jahr 1905 zeigt die beiden Jungvermählten in einem Passepartout (siehe Abbildung 1).

Die Vermählten sind nicht durch eine Fotografie abgebildet, sondern durch einen Künstler zeichnerisch festgehalten. Eingebettet ist die in schlichtem schwarz-weiß gehaltene Zeichnung in einem sich dem Betrachter öffnenden Vorhang. Die Zeichnung des Kronprinzen und seiner Braut schwebt in einem eigenen goldenen Rahmen eingefasst. Die Innenseite des Vorhangs ist bedeckt mit Hermelin, die Außenseite zeigt einen kräftigen Purpurton. Zu jeder Seite ist der Vorhang mit goldenen Kordeln gerafft, um die Vermählten dem öffentlichen Auge zu zeigen. Zu oberst des Umhangs thront die deutsche Kaiserkrone. In dieser Bildebene lassen sich zudem goldene Strahlen ausmachen, die von der Krone auszugehen scheinen. Im Hintergrund, der einen Farbverlauf von einem hellen Blauton zu einem Gelbton folgt, ranken sich die beblätterten Äste einer Eiche.

Gänzlich anders wurde hingegen die Hochzeit zwischen Prinz Olav V. von Norwegen (1903-1991) und Märtha von Schweden (1901-1954) im Jahr 1929 dargestellt (siehe Abbildung 2). Für das Motiv dieser Ansichtskarte, die die Prozession zum Altar zeigt, wurde ein tatsächliches Foto genutzt, das nicht wie im Falle des deutschen Kronprinzenpaares mit ornamentalem Beiwerk oder Zeichnungen geschmückt ist. Lediglich die Leuchtmittel im Innenraum der Kirche scheinen optisch dezent hervorgehoben. Abgesehen von dieser minimalen Bearbeitung handelt es sich um eine Momentaufnahme, die die wesentlichen Protagonisten von der hinteren Ansicht zeigt.

Zwischen den beiden Hochzeiten liegen knapp zwei Dekaden, in denen sich das Verständnis von Präsentation des herrschenden Adels veränderte, was diese beiden Ansichtskarten in einem leicht nachvollziehbaren Rahmen wiedergeben. Bei dem Beispiel der Hochzeit zwischen Olav V. und Märtha von Schweden liegt der Fokus auf dem Geschehen und dem Vorgang an sich und weniger auf den daran beteiligten Personen selbst. Der hier zu konstatierende Personenkult um die Regierenden ist jedoch kein Novum des 19. und frühen 20. Jahrhunderts, das erst eines Mediums wie der Ansichtskarte bedurfte, sondern setzte bereits mit der Etablierung der ersten Herrschaftsformen der 
Menschheit ein ${ }^{30}$ und entwickelte sich über die Jahrhunderte in verschiedenen Ausprägungen und Repräsentationsformen weiter, die bis hin zu Vorstellungen reichten, dass die Herrscher als Kopien der Götter auf Erden ${ }^{31}$ oder als eine Verkörperung eines übernatürlichen Wesens, das einen Körper beseelte, ${ }^{32}$ fungierten. Auch noch im 19. und beginnenden 20. Jahrhundert wurde die Idee des überragenden, übermenschlichen Herrschers vorangetrieben, besonders im Deutschen Kaiserreich, wobei auch hier bereits die Erziehung in einem solchen Verständnis bei den ersten Lehrversuchen der Kinder erfolgte - so etwa im Schulunterricht und in den dort eingesetzten Fibeln. ${ }^{33}$ Selbstredend verschwanden die Formen des Personenkults nicht mit dem Zusammenbruch der Monarchie in Deutschland oder Österreich ${ }^{34}$, sondern sind auch noch heute in diversen Formen zu beobachten. ${ }^{35}$

\section{Ein adeliges Leben verfolgbar durch die Ansichtskarte I- Die Hochzeit}

Stabilität und Kontinuität wurden nicht nur durch die Eheschließung an sich versprochen, sondern auch durch ihren tatsächlichen und langjährigen Fortbestand. So lassen sich nebst konkreter Hochzeitsansichtskarten auch Jubiläumskarten finden, wie anlässlich der Silberhochzeit des Deutschen Kaiserpaares im Jahr 1906 (siehe Abbildung 3).

In einem silbernen Kranz, unterbrochen durch ein Schild, das die Ehejahre aufzeigt, befinden sich monochrome Portraits von Wilhelm II. (1859-1941)

30 Vgl. Ernst Hartwig Kantorowicz: Götter in Uniform. Studien zur Entwicklung des abendländischen Königtums, (Bearb. Johannes Fried, Hrsg. Eckhart Gründwald, Ulrich Raulff, Stuttgart 1998. Insbesondere interessant ist dabei die wechselhafte Beziehung beziehungsweise Handhabe der Darstellung der Herrschenden und Göttern, was Ernst Kantorowicz in diversen Essays untersuchte und festhielt, dass »[...] die imitatio deorum von seiten der Kaiser [Kantorowicz bezieht sich hier auf die römischen Kaiser, bindet jedoch auch in einem anderen Abschnitt die Herrscher Ägyptens ein] durch eine imitatio imperatorum von seiten der Götter ergänzt.«, S. 61.

31 Ebd.

32 Vgl. Ernst Hartwig Kantorowicz: The King's Two Bodies. A Study in Medieval Political Theology, Princeton 2016.

33 Vgl. Gisela Teistler: Von der Kaiserverehrung zum Führerkult: Personenkult in Fibeln der Kaiserzeit und im Dritten Reich, in: Internationale Schulbuchforschung, 19 (3), Fibelgeschichte/History of primers, 1997, S. 285-304.

34 Vgl. Thomas Kunze, Thomas Vogel (Hrsg.): Oh Du, geliebter Führer. Personenkult im 20. und 21. Jahrhundert, Berlin 2013.

35 Exemplarisch seien hier die Kim-Dynastie in Nordkorea, sowie das enorme MerchandiseAufkommen des britischen Königshauses angeführt. Auch ist die Verlagerung des Personenkults des Adels auf andere Personen des öffentlichen Lebens, wie Schauspieler oder Musiker, interessant und zu erwähnen. 
und Auguste Viktoria (1858-1921), die jeweils in eine andere Richtung blicken. ${ }^{36}$ In einem zweiten Lorbeerkranz mit weißen Blüten oberhalb des Kaiserpaars ist der zum damaligen Zeitpunkt neu eröffnete Berliner Dom zu erkennen. Eine Germania, die einzig farbig gehaltene Person, hält in ihrer linken Hand ein mit Olivenblättern geschmücktes Schwert. Während die Germania leicht zum Kaiserpaar hinunterschaut und dabei ein dezentes Lächeln auf den Lippen auszumachen ist, hält sie in ihrer anderen Hand über das Portrait des Kaiserpaares einen kupfernen Kranz.

Weitere Olivenkränze erscheinen im linken, unteren Bereich der Ansichtskarte hinter einem Kissen, auf welchem die Herrscherkronen ${ }^{37}$ und ein Schwert niedergelegt sind.

Zwei Tauben im mittleren Bereich des Bildes scheinen miteinander zu interagieren und könnten das Herrscherpaar darstellen. Nebst der Tauben ist das Wappen Preußens niedergelegt, ebenso ein Wappen, das den preußischen Adler zeigt.

Kontinuität setzte bei den Zeitgenossen durchaus voraus, dass man sich seitens der Kartengestalter auf historische Vorkommnisse bezog, die im öffentlichen Gedächtnis präsent waren.

Auch Verbindungen, die bereits in einer weiter zurück liegenden Vergangenheit lagen, die für Zeitgenossen aber nach wie vor von historischer oder emotionaler Bedeutung waren, konnten folglich abgebildet werden. Als exemplarisches Beispiel sei an dieser Stelle die zweite Hochzeit Napoleon Bonapartes (1769-1821) angeführt, die 1810 mit Marie-Louise von Österreich (1791-1847) stattfand. Aus dieser Verbindung erhielt Napoleon seinen einzigen legitimen Sohn, Napoleon Franz Bonaparte (1811-1832) und bestärkte somit die Hoffnung einer Bonaparte-Dynastie. In einer Sammelreihe von Ansichtskarten, die die wesentlichen und einschneidenden Etappen in Napoleons Leben veranschaulichen ${ }^{38}$, ist auch jene Hochzeit grafisch festgehalten und wurde von keinem Zeitzeugen geschaffen. ${ }^{39}$ Der Szenenausschnitt der Ansichtskarte in Abbildung 4 zeigt Napoleon mit seiner rund zwei Jahrzehnten jüngeren Braut in Saint-Cloud während der Trauungszeremonie. Die Szenerie

36 Der abgewandte Blick ist ein Stilmittel, das sich auch häufig in Gemälden finden lässt. Ein in die Ferne gerichteter Blick kann als Protektion oder der Achtsamkeit gedeutet werden.

37 Auf der linken Seite die Kaiserkrone, auf der rechten Seite die Krone der Kaiserin, folglich sind die Kronen den Portraits entsprechend positioniert.

38 Auf der nun im Folgenden behandelten Ansichtskarte lässt sich beispielsweise die Zahl »21« in der rechten, unteren Ecke wiederfinden, die darauf hinweist, dass dies die einundzwanzigste Karte in dieser Themenreihe ist.

39 Auf der obersten Stufe vor dem Alter ist in Sichtrichtung des Betrachters eine Signatur erkennbar, »D. Mastroianni«, womit der italienische Künstler Domenico Mastroianni (1876-1962) gemeint ist. 
erweckt den Anschein der kirchlichen Hochzeit. Links unter Napoleons Füßen, scheinbar eingraviert in den Boden, ist deutlich zu lesen, dass es sich um Napoleons Hochzeit am 1. April handelt. An diesem Tag fand jedoch die zivile Hochzeit vom französischen Kaiser und seiner jungen Braut statt, erst einen Tag später wurde die kirchliche Trauung gefeiert. ${ }^{40}$

Das Motiv der Ansichtskarte wirkt bei näherer Betrachtung, als hätte der Künstler die Szenerie im kleinen Format in Ton modelliert und anschließend fotografiert.

\section{Ein adeliges Leben verfolgbar durch die Ansichtskarte II - Die Erben}

Im idealen Falle folgte auf die Hochzeit rasch der ersehnte Nachwuchs als Untermauerung der geschlossenen Verbindungen und, wie bereits erwähnt, als Garant für eine weitere Zukunft des Herrscher- oder Adelsgeschlechts.

Ansichtskarten bebilderten jegliche Varianten der jungen Adeligen, mit beiden Elternteilen, wie in einem Falle König Albert I. von Belgien (1875-1934) mit Gemahlin und Kindern (siehe Abbildung 5), nur einem Elternteil wie beispielsweise bei dem König der Sachsen, Friedrich August III. (1865-1932) (siehe Abbildung 6), lediglich mit ihren Geschwistern oder auch gänzlich allein.

Je nach Bedeutsamkeit ihrer Stellung lassen sich dabei Gefälle beobachten, sodass es einleuchtet, dass der einzige und letzte Zarewitsch Russlands, Alexei Nikolajewitsch Romanow (1904-1918), häufig alleine abgebildet worden ist (siehe Abbildung 7).

Doch auch Mehrgenerationen lassen sich ebenso als gewählte Motive auf Ansichtskarten wiederfinden. Da nicht zu jeder Zeit die wesentlichen Vertreter für eine Mehrgenerationsfotografie zugegen waren, oder der Fokus mitunter auf eine bestimmte Person gelegt werden sollte, waren bei der Bildbearbeitung und finalen Bildzusammenstellung nicht selten einige Kniffe unternommen worden. Durch solche Zusammenstellungen entstand folglich die Möglichkeit mehrere Generationen in einem Bildteil aufzubauen, wie einen klassischen Stammbaum (siehe Abbildung 8) oder schlicht Personen in das Bild einzufü-

40 Vgl. Maria Anna Flecken: Marie Louise von Österreich: „Concordia« oder die Fantasie der Einheit Italiens? Mehrdeutigkeit als politisches Gestaltungsmittel im Werk Antonio Canovas, o. A. 2008, S. 4. 
gen, sodass Paare gebildet wurden, die zum Zeitpunkt der einzelnen Aufnahmen nicht zugegen waren. ${ }^{41}$

\section{Ein adeliges Leben verfolgbar durch die Ansichtskarte III - Militärische Karriere}

Ein weiterer chronologischer Schritt ${ }^{42}$, der eine erhöhte Produktion von Ansichtskarten bedeutete, waren kriegerische Auseinandersetzungen, Darstellungen des Militärs und errungener Siege. ${ }^{43}$ In dem hier betrachteten Zeitraum ist vor allem der Erste Weltkrieg auch für die Motivik der Ansichtskarten von entscheidender und weitreichender Bedeutung.

Der Erste Weltkrieg war aus medialer Sicht ein Gipfelpunkt der Propaganda. Die Ansichtskarte war ein gerne genutztes Medium, um einerseits die Massen $\mathrm{zu}$ erreichen und diese von den Vorstellungen des Adels zu überzeugen und andererseits, um Unterstützungen, finanzieller oder ideeller Art, aus der Bevölkerung zu erhalten. Bewirkt werden sollte dies durch die Präsentation der heroischen Leistungen des Militärs, die im Nachhinein vornehmlich Einzelpersonen aus dem Kreis der Adeligen zugesprochen wurden (siehe Abbildung 9).

Vermeintliche Zitate, versehen mit abgedruckten Unterschiften und somit direkten Aufrufen an den Betrachter, dienten als propagandistisches Gestaltungsmittel. So finden sich beispielsweise auf Ansichtskarten aufgedruckte Aufrufe der deutschen Kaiserin Auguste, mit welchem sie sich an die deutschen Frauen richtete und deren Unterstützung einforderte (siehe Abbildung 10). ${ }^{44}$

Auch dienten in diesem Zusammenhang häufig Fotografien zerstörter Ortschaften, Wohnhäuser oder Kirchen als Motiv für entsprechende Ansichtskarten. Selbstredend konnte es sich bei diesen Fotografien lediglich um Bestands-

41 Zum Themenbereich der Reproduktion und Veränderung des Bildmaterials siehe in diesem Beitrag im späteren Abschnitt.

42 Chronologie im Sinne der klassischen Lebensabschnitte eines Adeligen.

43 Siegesdarstellungen sind jedoch kein Novum der Ansichtskarte des 19. oder frühen 20. Jahrhunderts, sondern gehören zum wesentlichen Instrument der visualisierten Machtansprüche und Untermauerungen jener. So lassen sich über Jahrhunderte Siegesdarstellungen unter anderem auf Münzen, Tapisserien, Gemälden oder Architektur wiederfinden.

44 Allgemein lässt sich die Wohltätigkeit häufig als thematisches Motiv auf Ansichtskarten finden, nicht nur während Kriegszeiten, sondern auch in der Nachsorge der Kriegsversehrten. In Preußen gab es bereits um 1900 seit einigen Jahrzehnten eine Vereinigung, die sich die Unterstützung der Kriegsfürsorge als Aufgabe gesetzt hatte. Der Vaterländische Frauenverein wurde 1866 von der damaligen Königin Preußens, Augusta (1811-1890), gegründet, Vgl. Marget Schulz: Frauen machen sich auf den Weg. Eine Dokumentation zur Geschichte der Frauen in Maintal, Hanau 1994, S. 93. 
aufnahmen handeln. Dennoch gibt es durchaus eine zusätzliche Bedeutungsebene, durch die diese Aufnahmen in Verbindung zum Adel gebracht werden konnten. Diese Abbildungen bezeugten die wortwörtliche Schlagkraft des Militärs und konnten somit ein Gefühl des Sieges vermitteln. Auf der anderen Seite konnte es allerdings auch als Abschreckung und zugleich als Anstachelung dienen, um den Feind an weiteren Zerstörungen zu hindern. Fraglich ist, wie präsent, in Form der Ansichtskarten, die jeweiligen Adelshäuser in den verfeindeten Ländern waren. Wenn sich überhaupt Darstellungen der anderen europäischen Königshäuser im Deutschen Kaiserreich zur Zeit des Ersten Weltkriegs wiederfinden lassen, stehen sie doch in der Regel in einem direkten Bezug zu einem Verbündeten- oder Feindbild und zeigen damit einhergehende Attribute. ${ }^{45}$ Studien, die sich damit jedoch eingehend beschäftigt haben, liegen bisher nicht vor.

\section{Ein adeliges Leben verfolgbar durch die Ansichtskarte IV - Tod und Glorifizierung}

Zahlreiche Tote waren auf jeder Seite des Krieges zu beklagen und selbst für die öffentliche Trauer wurde die Ansichtskarte als Medium genutzt. Auffällig ist dabei, dass vor allem künstlerische Karten dafür angefertigt wurden und keine Fotografien. ${ }^{46}$ Auch der Monarch starb, auf natürliche Weise wie Alter oder Krankheit, oder auch durch einen Gewaltakt, beziehungsweise einen Unfall. König Humbert von Italien, oder Umberto I. (1844-1900), erlag den Verletzungen, die ihm durch einen von einem Anarchisten abgefeuerten Revolver zugefügt worden waren, wie dies durch einen diesbezüglichen Schriftzug auf der entsprechenden Ansichtskarte (siehe Abbildung 11) betont wird. Nicht nur wird dieser unnatürliche Tod durch Schrift auf einer Ansichtskarte kommentiert.

Die Kränze erinnern an einen Lorbeer- oder Palmzweigkranz, wodurch eine Stilisierung des italienischen Königs zu einer Art Märtyrer stattfindet.

45 Studien, die sich mit den damit zusammenhängenden Fragen befassen, liegen jedoch bislang nicht vor. In der Historie ist der Verbündete in der Regel als gleichgestelltes, heroisches Individuum porträtiert, der Feind wird dagegen häufig karikiert und überzogen dargestellt, seine Handlungen werden mehr tierisch als menschlich klassifiziert. Verschmähende Darstellungen verfeindeter Gruppen oder Einzelpersonen weisen eine Jahrhunderte alte Tradition auf, wobei man den ersten Höhepunkt in der Zeit der Reformation mit dem Konflikt zwischen Katholiken und Protestanten sehen kann.

46 Interessant ist dabei auch ein offenbarer geschlechtsspezifischer Unterschied, so obliegt es dem deutschen Kaiser sein direktes Bekümmern auszudrücken durch »Ich habe es nicht gewollt«, doch im Falle der Kaiserin wird in der Regel den Verstorbenen ein »treues Andenken« versichert. 
Bei der ersten Karte von den bisher behandelten ist auf jener von König Humbert eine Möglichkeit der Anzahl der Drucke zu erkennen. Dieses Faktum ist dahingehend von Bedeutung, da es Aufschluss über eine Mindestangabe von Rezipienten gibt und im Zuge dessen eine Aussagekraft über eine begehrte Motivik. In der unteren rechten Bildebene ist ein »Dess. No. 732« abgedruckt, was sehr wahrscheinlich die Nummer des Dessins aufzählt. Ungewiss ist jedoch, ob sich diese Aufzählung auf das Dessin bezeichnet, das lediglich mit König Humbert in Verbindung zu bringen ist, oder generell eine Dessinnummer des Verlags, der die hier angesprochene Ansichtskarte veröffentlichte. Der Verlag ist auf der unteren linken Bildebene verzeichnet. ${ }^{47}$ Es handelt sich um eine häufige Begebenheit im Falle der Ansichtskarten, dass der Verlag auf ihrem Produkt verzeichnet worden ist, doch in der Regel ist dies die einzige Information, die zu den Produzenten noch zu finden ist. Vereinzelt lassen sich Zusatzinformationen finden, wie der Hinweis, dass die Abbildungen rechtlich geschützt sind ${ }^{48}$, die schlichte Adresse des Verlags oder der Fotograf und sein Status. ${ }^{49}$

Solchen Ansichtskarten, die zumindest ein kleines Maß an Informationen vermitteln, stehen solche gegenüber, die lediglich die bildliche Information transportieren und nichts auf den Verlag, Fotografen oder ähnliches hindeutet. Ein solcher Fall einer äußerst knappen Informationslage anhand einer Ansichtskarte stellt ein Exemplar mit der Abbildung des verstorbenen Königs Otto von Bayern (1848-1916) dar. Otto von Bayern, ein auf Grund seiner psychischen Schwierigkeiten unfähiger Regent, ${ }^{50}$ erlag einer Darmerkrankung,

47 »Max Marcus, Berlin W.«.

48 »Als Postkarte offen hinterlegt beim Königl. Amtsgericht. Jede Nachahmung wird gerichtlich verfolgt«, am Beispiel einer Ansichtskarte, die Kronprinz Wilhelm und seine Braut Cecilie anlässlich ihrer Vermählung zeigen, Vgl. Michael Meyer: Welsch Huus - Mehr als eine Kronenburger Familiengeschichte. Teil I Von den Anfängen bis zum Zweiten Weltkrieg, Norderstedt 2019, S. 163.

49 Status meint in diesem Sinne ob der Fotograf sich offiziell als Hoffotograf bezeichnen darf. Bedauerlicherweise scheint es in dieser Richtung noch keinerlei Forschungsvorhaben gegeben zu haben, weswegen das Verlagswesen der Ansichtskarten recht unerforscht ist. Bei dem Berliner Ansichtskartenverlag Gustav Liersch \& Co, nach der Adresssetzung im Kaiserreich zu lokalisieren in Berlin S.W. 48, Friedrichstraße 16, beispielsweise handelt es sich wahrscheinlich um den größten Produzenten von Ansichtskarten, die die Hohenzollern porträtierten. Die Ansichtskarten waren offiziell per Gesetz vor fremder Vervielfältigung geschützt. Dieser Verlag schien ein regelrechtes Monopol der Ansichtskartenherstellung gehabt zu haben, da sich auch der Zusatz des Hoffotografen wiederfinden lässt und somit die direkte Auftragsvergabe und Bestätigung ersichtlich ist. Umso bedauerlicher ist das bisherige Fehlen einer wissenschaftlichen Auseinandersetzung, besonders im Bezug auf die Vorgaben, Absprachen und Freiheiten, die mit der Vertragsaufgabe zwischen dem Adel und dem Verlag bestanden haben müssen.

50 Vgl. Thomas Köhler: Ruhm \& Wahnsinn. Psychische Störungen bekannter Persönlichkeiten, Stuttgart 2017, S. 99-103. 
wobei die Aufbahrung des Königs durch Fotografien dokumentiert wurde (siehe Abbildung 12).

Eine offensichtlich nachträglich eingefügte Schrift erläutert den Umstand der Entstehung der Fotografie, doch ansonsten steht die Abbildung für sich. Fotografien von Verstorbenen waren keine Seltenheit, sondern hatten bereits seit dem 19. Jahrhundert eine lange Tradition. ${ }^{51}$ In der Regel wurde der Körper so drapiert, dass es wirkte, als ob der Verstorbene sich eher in einem Schlaf befinden würde, als tatsächlich tot zu sein. Dies lässt sich auch auf die angefertigte Fotografie von Otto von Bayern übertragen, die als Motiv einer Ansichtskarte genutzt wurde. Selbstredend diente eine solche Ansichtskarte, die den verstorbenen Monarchen zeigte, der Memoria, einer letzten Ehrung und Erinnerung. Als Kaiserin Auguste Viktoria 1921, also drei Jahre nach dem Zerfall der Monarchie in Deutschland, verstarb, erfolgte eine regelrechte Flut an neu gedruckten Ansichtskarten, die ihrem Andenken gewidmet waren. Nicht nur Fotografien, die Jahre vor der erzwungenen Abdankung der Kaiserin angefertigt wurden, kamen erneut auf den Markt, sondern auch solche, die den Trauerzug dokumentierten. Augustes sterbliche Überreste wurden aus dem niederländischen Exil nach Deutschland überführt. In einer ausladenden Prozession wurde der Sarg durch Berlin in den Antikentempel im Park zum Schloss Sanssouci getragen. Rund 200.000 Menschen sollen an der Prozession beteiligt gewesen sein. ${ }^{52}$

Diese große Anteilnahme ist nicht nur mit dem tatsächlichen Wesen der Kaiserin zu begründen, sondern auch auf Grund der öffentlichen Meinung, die unter dem Volk über Auguste verbreitet wurde, wobei auch in diesem Falle Ansichtskarten eine tragende Rolle übernommen haben könnten. Auguste wurde als Mutter der Nation stilisiert, ${ }^{53}$ interessiert an Wohltätigkeit und Fürsorglichkeit, eine gute Mutter, die vielen Söhnen das Leben schenkte und gottesfürchtig war.

Eine Ansichtskarte, die die Kaiserin Auguste als eine solche Landesmutter wörtlich deklariert, zeigt sie vor einem dunkelroten Vorhang (siehe Abbildung 13).

51 Siehe dazu beispielsweise Katharina Sykora: Die Tode der Fotografie. Totenfotografie und ihr sozialer Gebrauch, Bd. 1 und 2, München 2017 oder auch Isabel Richter: Der phantasierte Tod: Bilder und Vorstellungen vom Lebensende im 19. Jahrhundert, Frankfurt am Main 2010.

52 Vgl. Karin Feuerstein-Praßer: Die deutschen Kaiserinnen 1871-1918, Regensburg 1997, S. 235. Abgesehen von der großen Anteilnahme in der Heimat organisierte die niederländische Regierung alle Flaggen an Bahnhöfen auf Halbmast zu senken, durch die der Zug mit Augustes sterblichen Überresten fuhr, Justin C. Vovk: Imperial Requiem. Four Royal Women and the Fall of the Age of Empires, Bloomington 2012, S. 418.

53 Franz-Josef Pütz klassifiziert ihre Präsenz auf Ansichtskarten als Mutter der Nation als eine Erfüllung ihrer auszuführenden, medialen Rolle, vgl. Franz-Josef Pütz: Das Ringen um Gefühle - Ansichtskarten im Ersten Weltkrieg, in: Michel-Rundschau, 2014 (9), S. 49. 
Zur Linken kann der Betrachter einen kleinen Ausschnitt einer Landschaft ausmachen, die vornehmlich gekennzeichnet ist durch Industrieanlagen, die Flagge des Deutschen Roten Kreuz ist ebenfalls in diesem Abschnitt zu erkennen. Augustes wertvolle Kleidung und Schmuck stehen im starken Kontrast zu den ärmlich gekleideten, teils zerlumpten beiden Kindern, die sie umrahmen. An ihrer linken Seite steht ein Junge, der zur Kaiserin nach oben blickt. Seine linke Hand berührt die Augustes, die sich auf der Schulter des Jungen befindet. Mit der anderen Hand hält der Junge eine Brotscheibe. Ein kleineres Mädchen steht auf der anderen Seite der Kaiserin. Auf Grund ihrer geringeren Körpergröße ruht Augustes Hand nicht auf der Schulter des Kindes, sondern berührt die Wange des Mädchens. Mit ihren kleinen Händen umschlingt das Mädchen einen kleinen Laib Brot. Ihr Blick ist jedoch weder auf das Brot, noch auf die Kaiserin gerichtet, mit ihren traurigen Augen schaut das Mädchen den Betrachter direkt an. Obwohl die Kaiserin in unmittelbarer Nähe zu den Kindern steht, geht ihr Blick nicht zu ihren Schützlingen runter, sondern ist in die Ferne gerichtet und führt am Betrachter vorbei. Die Szenerie wird abgeschlossen durch eine Inschrift, "Unsere Landesmutter", auf einer Art Marmor- oder anderen Steinplatte. Diese Steinplatte ist abgeschlossen mit einem vegetabilen Band. Zwei schlichte, ebenso steinerne Blüten verzieren die Platte neben der Inschrift. Interessanterweise lässt sich auf der Köthener Brücke in BerlinKreuzberg eine ähnliche Blüte finden. Die Köthener Brücke wurde zwischen 1909 und 1910 errichtet, hat unter sich den Landwehrkanal fließen und verband zum Zeitpunkt der Errichtung zwei eigenständige Städte, Berlin und den nun zur Hauptstadt gehörenden Bezirk Schöneberg. ${ }^{54}$ Zwei unterschiedliche Blumenmotive lassen sich auf der Köthener Brücke finden, wobei die erste Variante (siehe Abbildung 14) der Blüte ${ }^{55}$ auf der Ansichtskarte ähnelt, sodass ein interessanter Bezug, beziehungsweise eine interessante Wechselbeziehung, zwischen der Kunst auf einer Ansichtskarte und der Dekoration einer Alltagsarchitektur vorhanden sein kann. Solche Blumenmotive lassen sich zur Zeit des Jugendstils häufig als Gestaltungselement wiederfinden. Dem Geschmack der Zeit wurde dementsprechend auch bei den Ansichtskarten des Adels gefolgt.

Die andere Seite der just behandelten Ansichtskarte gibt nähere Auskunft über die Entstehung des Motivs. Das originale Gemälde, das als Vorlage für die

54 Die Brücke ist Teil der Denkmaldatenbank des Landesdenkmalamts in Berlin, http://www .stadtentwicklung.berlin.de/denkmal/liste_karte_datenbank/de/denkmaldatenbank/daobj.php? obj_dok_nr=09031182 (letzter Zugriff: 14.05.2019).

55 Die Blüte selbst wirkt wie eine Blumenrosette oder eine stilisierte Chrysantheme. Auch noch an einer anderen Berliner Brücke lässt sich diese Blüte wiederfinden, nämlich am Wandbrunnen der Eisernen Brücke in Berlin-Mitte, die 1914-1916 mit diesem Design errichtet wurde. 
Druckversion der Ansichtskarte genutzt wurde, schuf einer der Hofmaler Kaiser Wilhelms II. - der Berliner Künstler Arthur Fischer (1872-1948). Fischer nutzte für seine Arbeiten im ersten Schritt Fotografien, damit seine Modelle nicht allzu lange stillstehen oder in ihrer Position verharren mussten. Folglich war in der Regel das eigentliche Model gar nicht zugegen, als das Gemälde geschaffen wurde. Interessanterweise nutzte Fischer die Technik der Fotografie, um im nächsten Schritt auf Basis dieser Wiedergabe eine Reproduktion in klassischer Weise zu erschaffen. Die Fotografie diente damit dem gleichen Zweck wie die sonst üblichen Vorzeichnungen. Fischers Spezialität war die genaue Reproduktion. Er verstand sich somit nach eigener Aussage eher als ein hervorragender Kopist, denn als ein großartiger Künstler. Wobei er betonte, dass er auf diese Weise seine Aufträge stets in kürzester Zeit abschließen konnte. ${ }^{56}$

Nicht nur die Technik, mit der Arthur Fischer dieses Ansichtskartenmotiv schuf, ist klassisch, er bediente sich auch eines Stilmittels, das seit Jahrhunderten vornehmlich eingesetzt wurde, um Neugierde zu erwecken. Um den Hintergrund größtenteils zu verhüllen, nutzte Fischer einen Vorhang. Vorhänge haben in der klassischen Malerei eine lange Tradition, wie etwa bei Plinius dem Älteren (23-79) in seiner Naturalis Historia festgehaltenen Legende, in der Zeuxis und Parrhasius von Ephesus (beide 5. Jahrhundert v. Chr.) darum wetteiferten, wer der bessere Künstler wäre. Parrhasius schuf ein derart realistisches Bild eines Vorhangs, dass Zeuxis seinen Gegner dazu aufforderte den Vorhang zu lüften, damit er schließlich Parrhasius' eigentliches Werk sehen könne. ${ }^{57}$

Vorhänge als Bestandteil eines Gemäldes erhielten eine Wiederbelebung während der Renaissance, vor allem als Detail in Portraits. Den Adel in klassischen Gemälden zu porträtieren folgte einfachen, doch fest bestimmten Regeln. Die Person sollte in einer Umgebung präsentiert werden, die gewährleistet, dass Würde, Intelligenz, Gnade oder welche konkreten Eigenschaften sonst im Gemälde festgehalten und präsentiert werden sollten, auch wiedergespiegelt werden konnten. Diese Art und Weise ist vergleichbar mit den Bebilderungen der Ansichtskarten, denn auch diese folgten einem Regelwerk, um den Adel von seiner besten Seite zu zeigen.

Der Adel wurde durch Ansichtskarten nicht nur so präsentiert, wie das Volk ihn sehen wollte, sondern auch wie der Adel gesehen werden musste, um dem sozialen und gesellschaftlichen Stand gerecht zu werden, den er auch in der Wilhelminischen Zeit noch verkörperte. Um diesen Anforderungen gerecht zu

56 N.N.: Plünderer sind in Lebensgefahr, in: Der Spiegel, 1947 (6), S. 16, http://www.spiegel.de /spiegel/print/d-39344260.html.

57 Vgl. Plinius: Nat. Hist., XXXV, S. 64-65. 
werden, musste notfalls auch mit Retuschen nachgeholfen werden. Ein extremes Beispiel für eine solche Bildfälschung sind Darstellungen der österreichische Kaiserin Elisabeth (1837-1898). Es existiert lediglich eine einzige Fotografie, die sie zusammen mit ihrem Ehemann, Franz Josef von Österreich, und ihren beiden Kindern, Erzherzogin Gisela (1856-1932) und Kronprinz Rudolf (1858-1889) zeigt, 1859 durch den aus der Slowakei stammenden österreichischen Fotografen Ludwig Angerer (1827-1879) aufgenommen. ${ }^{58} \mathrm{Da}$ anderes Material nicht als Vorlage dienen konnte, mussten vielerlei Retuschen angefertigt werden, um für verschiedene Anlässe entsprechende Bilder zu generieren. Gleiches geschah auch im Falle des deutschen Kaiserhauses, auch wenn, im Vergleich zu den Habsburgern, deutlich mehr Ausgangsmaterial zur Verfügung stand, das für solche Zwecke genutzt werden konnte.

Häufig, wenn auch nicht immer eindeutig, ist auch nach der Retusche zu erkennen, welches Bild oder welche Fotografie als Ausgangsobjekt genutzt worden war. So war es durchaus möglich, dass aus einem grafisch gehaltenen Motiv wie in Abbildung 16, die Reproduktion eines vermeintlichen Porträts entstand (siehe Abbildung 17), in welcher der Kaiserin zudem ein gänzlich anderes Objekt in die Hand gelegt wurde, denn anstelle des kleinen Fächers, den sie im Original in Händen hält, wurde Auguste in einer der vielfältigen Variationen ein kleiner Blumenstrauß in die Hände drapiert. Darüber hinaus konnten Objekte und Personen jedoch auch gänzlich gedreht und neu hinzugefügt (siehe Abbildung 18) oder auch abgeschnitten ${ }^{59}$ und vergrößert werden (siehe Abbildung 19 und 20).

Wenige vorhandene Motive konnten auf diese Weise unzählige Male neu genutzt, umgedeutet, gedreht oder beschnitten werden. Die Idee ${ }^{60}$, eine Person nach bestimmten Vorgaben wiederzugeben, gab es bereits seit Jahrhunderten, doch im Laufe des 19. und 20. Jahrhunderts wurden die Techniken vereinfachter und, wenn man möchte, salonfähig. ${ }^{61}$

58 Vgl. Fischer-Westhauser, Mraz 1998, S. 103.

59 Häufig lassen sich auch Ansichtskarten finden, die durch eine Fotografie ein repräsentatives Gebäude, oder eine andere markante Architektur zeigen. Da sich diese in der Regel im innerstädtischen Bereich befanden, ist das Vorhandensein vieler Menschen unabdingbar und nachvollziehbar. Für die Ansichtskartenmacher störten jedoch die sogenannten alltäglichen Menschen, weswegen häufig einige Passanten aus dem Bild herausgeschnitten wurden. Dieses Wegretuschieren in einer Fotografie hinterließ Schraffierungen, sodass es durchaus sichtbar war, dass zuvor dort noch ein Mensch, Pferd oder Karren gestanden hatte. Obwohl diese Veränderungen teilweise derart sichtbar waren, dass dadurch das Auge eigentlich noch eher zu diesem Punkt gezogen werden müsste, wurden sie dennoch durchgeführt und so in den Druck geschickt.

60 Der Begriff Idee im Sinne von Tradition.

61 Wie bereits zu Beginn erläutert beschränkten sich die visuellen Wiedergaben der Herrschenden auf Objekte, die mitunter dem gemeinen Volk nur im geringen Maße zur Verfügung standen. 


\section{Der perfekte Schein - Retusche und Voyeurismus}

Ebenso salonfähig war seit den Kabinett- und Carte-de-Visite-Formaten die Illusion einen individuellen Blick auf eine sonst scheinbar unerreichbare Person zu werfen oder zu erhaschen, wie zum Beispiel auf die Kaiserin beim Lesen (siehe Abbildung 21).

Auch wenn deutlich zu erkennen ist, dass es sich bei Aufnahmen um eindeutige Inszenierungen handelt, beispielsweise im Falle einer lesenden Kaiserin, die während der privaten Lektüre ihre Krone auf dem Haupt trägt (siehe Abbildung 22), scheinen sie dennoch einen Absatz gefunden zu haben, die erlaubten solche Ansichtskarten über Jahre zu veröffentlichen.

Die Möglichkeiten der Bebilderung der Ansichtskarten zeichnen sich durch ihre Variabilität aus. Die Nachfrage zu solchen Abbildungen war groß, was den Wunsch der Untertanen nach Informationen unterstrich - Informationen, die bezeugten, dass es sich um eine intakte Familie und eine intakte Monarchie handelte. Ein Voyeurismus wurde bedient. Selbstredend war nicht das Volk die wesentliche, treibende Kraft bei der Herstellung der Ansichtskarten, sondern der Adel selbst. Jedes Bild, jede Handlung konnte perfekt inszeniert werden. Schwächen, wie politische Fehlentscheidungen, körperliche und geistige Missbildungen konnten schlicht kaschiert werden. In dieser Art ist die Kunst der Ansichtskarte vergleichbar mit den klassischen Medien der Kunst. Auch in Gemälden und grafischen Werken wurden die Schwächen des Adels versteckt und dafür ihre besonders positiven Eigenschaften hervorgehoben oder solche konstruiert. All die unterschiedlichen Variationen der Reproduktion haben gemein, dass sie von Menschenhand gemacht sind, sodass alles im Sinne des Auftraggebers inszeniert werden kann. Ansichtskarten hatten gegenüber Gemälden den Vorteil, dass sie in einer deutlich höheren Stückzahl und zudem in einer Geschwindigkeit produziert werden konnten und können, die bei Gemälden nicht möglich war. Aber dennoch bewahrt sich die Ansichtskarte etwas durchaus künstlerisches.

\section{Schlussbetrachtung}

Zusammenfassend muss festgehalten werden, dass der Adel auf Ansichtskarten so dargestellt ist, wie er von der Bevölkerung gesehen werden wollte, aber auch musste, um seiner gesellschaftlichen Stellung zu entsprechen. Anders ausge-

Salonfähig meint folglich in diesem Zusammenhang die zunehmende Vervielfältigung von visuellen Reproduktionen und der damit vereinfachte Zugang an Bildmaterialien, eben auch für die sogenannte einfache Bevölkerung. 
drückt bedeutete dies die Darstellung einer perfekten Erfüllung der klassischen Rollenverteilungen. Die Frau wurde als Stütze des Mannes dargestellt, die als Mutter der folgenden Generation den Fortbestandes des Adelsgeschlechts sicherte und die sich für die Belange der Bevölkerung interessierte und sich im besten Falle in Form von Hilfsorganisationen engagierte und somit zur Landesmutter avancierte. Der Mann dagegen wurde als die treibende Kraft und als Beschützer, zumeist in militärischer Funktion, dargestellt. Dass die Kinder in die wortwörtlichen Fußstapfen zu treten hatten, ist besonders im Falle der Jungen ersichtlich, wenn diese bereits im Kleinkindalter stolz in militärischen Uniformen posierten.

Auch wenn die Ansichtskarte ein noch verhältnismäßig neues oder junges Medium um 1900 war, konnten alte und neue Wege der Präsentation genutzt werden, um einen Blick auf den Adel zu gewährleisten. Traditionen, die den klassischen Darstellungen auf Gemälden, Münzen, Kupferstichen oder ähnlichem folgten, wurden zwar übernommen, aber auch um die neueren technischen Methoden ergänzt. Als eine schlichte Kopie der altehrwürdigen Kunst mit ein paar wenigen neuen Kniffen sollte die Ansichtskarte somit nicht angesehen werden, gewährleistete sie doch zum Teil die Sicht auf den Menschen hinter der Maske des Adels. Ein Novum der Ansichtskarten ist die Darstellung von Vertrautheit, sei es in der familiären Umgebung oder der Vertrautheit des Ortes, was durch Schnappschussfotografien, eine frühe Form der Paparazzi, erreicht wurde.

Die Möglichkeiten des Collagierens und der Retusche sind im Bereich der Ansichtskarten unbegrenzt. Mit diesen Bildveränderungen kann die Ansichtskarte als ein Photoshop des 19. Jahrhunderts bezeichnet werden und reiht sich zudem in eine Kontinuität der Bildveränderung ein. Besonders in diesem Bereich gibt es in der Forschung noch erhebliche Lücken. Der Ansichtskarte wurde in der Kunstgeschichte als Quellenmaterial bisher kein umfangreicher Platz zugesprochen, da sie meist schlicht als Propaganda oder Kitschobjekt in eine Vorurteilsecke abgeschoben wird. ${ }^{62}$ Die bisher vorhandene Forschungsliteratur setzt sich in der Regel mit dem Kaiserreich oder dem Militarismus in der ersten Hälfte des 20. Jahrhunderts auseinander, doch bietet dieses Feld durchaus mehr, was hoffentlich in den kommenden Jahren erkannt wird.

62 Vgl. Lebeck und Kaufmann 1985, S. 191 und S. 421 sowie May 1998 S. 89. 


\section{Literaturverzeichnis}

Samuel Edgerton: Pictures and Punishment. art and criminal prosecution during the Florentine Renaissance, Ithaca 1985 oder Richard Ward: Print Culture, Crime and Justice in Eighteenth-Century London, London 2014.

Karin Feuerstein-Praßer: Die deutschen Kaiserinnen 1871-1918, Regensburg 1997.

Ulla Fischer-Westhauser, Gerda Mraz: Elisabeth. Prinzessin in Bayern, Kaiserin von Österreich, Königin von Ungarn. Wunschbilder oder die Kunst der Retouche, Wien 1998.

Maria Anna Flecken: Marie Louise von Österreich: »Concordia« oder die Fantasie der Einheit Italiens? Mehrdeutigkeit als politisches Gestaltungsmittel im Werk Antonio Canovas, o. A. 2008.

Günter Formery, Thomas Fürst: Die Welt des Ansichtskartensammelns, Schwalmtal 2011.

Karen A. Foss, Stephen W. Littlejohn, John G. Oetzel: Theories of human communication, Long Grove 2017.

Siegfried Frey: Die Macht des Bildes. Der Einfluß der nonverbalen Kommunikation auf Kultur und Politik, Bern 1999.

Walter Goldschmidt: Nonverbal Communication and Culture, in: Ullica Segerstråle, Peter Molnár (Hrsg.): Nonverbal communication. Where nature meets culture, Mahwah 1997), S. 229-244.

Ernst Hartwig Kantorowicz: Götter in Uniform. Studien zur Entwicklung des abendländischen Königtums, (Bearb. Johannes Fried, Hrsg. Eckhart Gründwald, Ulrich Raulff, Stuttgart 1998.

Ernst Hartwig Kantorowicz: The King's Two Bodies. A Study in Medieval Political Theology, Princeton 2016.

Christine Haug: Reisen und Lesen im Zeitalter der Industrialisierung. Die Geschichte des Bahnhofs- und Verkehrsbuchhandels in Deutschland von seinen Anfängen um 1850 bis zum Ende der Weimarer Republik, Wiesbaden 2007.

Lutz Hieber: Gesellschaftsepochen und ihre Kunstwelten, Wiesbaden 2018.

Thomas Köhler: Ruhm \& Wahnsinn. Psychische Störungen bekannter Persönlichkeiten, Stuttgart 2017.

Thomas Kunze, Thomas Vogel (Hrsg.): Oh Du, geliebter Führer. Personenkult im 20. und 21. Jahrhundert, Berlin 2013.

Robert Lebeck, Gerhard Kaufmann: Viele Grüße...Eine Kulturgeschichte der Postkarte, Dortmund 1985.

Otto May: Deutsch sein heißt treu sein. Ansichtskarten als Spiegel von Mentalität und Untertanerziehung in der Wilhelminischen Ära (1888-1918), Hildesheim 1998.

Otto May: Vom Wachsen lassen zum Führen. Die Ansichtskarte als Zeuge einer versäumten Erziehung zur Demokratie in der Weimarer Republik, Hildesheim 2003.

Otto May: Inszenierung der Verführung. Die Ansichtskarte als Zeuge einer au-toritären Erziehung im III. Reich, Hildesheim 2003.

William McAllister Johnson: Numismatic propaganda in Renaissance France, in: Detroit Institute of Arts (Hrsg.): The art quarterly, 31, Detroit 1968, S. 123-153.

Michael Meyer: Welsch Huus - Mehr als eine Kronenburger Familiengeschichte. Teil I Von den Anfängen bis zum Zweiten Weltkrieg, Norderstedt 2019. 
Jutta Nowosadtko: Hinrichtungsrituale. Funktion und Logik öffentlicher Exekutionen in der Frühen Neuzeit, in: Michael Matheus, Sigrid Schmitt (Hrsg.): Kriminalität und Gesellschaft in Spätmittelalter und Neuzeit, Stuttgart 2005, S.71-94.

Franz-Josef Pütz: Das Ringen um Gefühle - Ansichtskarten im Ersten Weltkrieg, in: MichelRundschau, 2014 (9), S. 47-53.

Heinz Reif: Adel im 19. und 20. Jahrhundert, München 2012.

Uli Reiter: Illegalität. Phänomen und Funktion, Wiesbaden 2016.

Isabel Richter: Der phantasierte Tod: Bilder und Vorstellungen vom Lebensende im 19. Jahrhundert, Frankfurt am Main 2010.

Charles M. Rosenberg, Money talks. Numismatic propaganda under Alfonso I d'Este, in: Angela Ghinato (Hrsg.): L' età di Alfonso I e la pittura del Dosso, Modena 2004, S. 145164.

Johannes Rüberg: Die Preisbestimmung in der Telekommunikation, Tübingen 2017.

Marget Schulz: Frauen machen sich auf den Weg. Eine Dokumentation zur Geschichte der Frauen in Maintal, Hanau 1994.

Katharina Sykora: Die Tode der Fotografie. Totenfotografie und ihr sozialer Gebrauch, Bd. 1 und 2, München 2017.

Gisela Teistler: Von der Kaiserverehrung zum Führerkult: Personenkult in Fibeln der Kaiserzeit und im Dritten Reich, in: Internationale Schulbuchforschung, 19 (3), Fibelgeschichte/History of primers, 1997, S. 285-304.

Justin C. Vovk: Imperial Requiem. Four Royal Women and the Fall of the Age of Empires, Bloomington 2012.

Karin Walter: Die Ansichtskarte als visuelles Massenmedium, in: Wolfgang Kaschuba, Kaspar Maase (Hrsg.): Schund und Schönheit. Populäre Kultur um 1900, Köln 2001, S. 46-61.

Reinhard Wolters: Die Geschwindigkeit der Zeit und die Gefahr der Bilder: Münzbilder und Münzpropaganda in der römischen Kaiserzeit, in: Gregor Weber, Martin Zimmermann (Hrsg.): Propaganda - Selbstdarstellung - Repräsentation im römischen Kaiserreich des 1. Jhs. n. Chr., Wiesbaden 2003, S. 175-204. 


\section{Abbildungen}
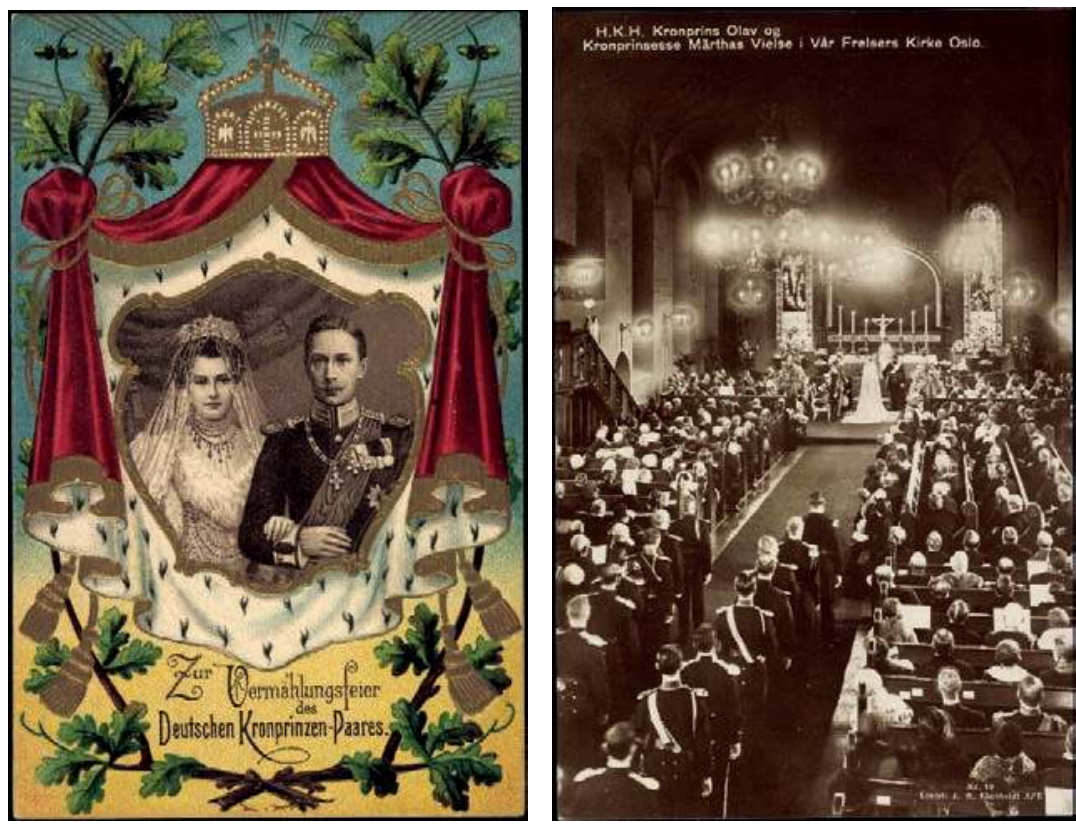

Abbildung 1-2: Ansichtskarte anlässlich der Hochzeit des deutschen Kronprinzen mit Cecilie von Mecklenburg, 1905 und Ansichtskarte anlässlich der Hochzeit von Prinz Olav V. von Norwegen mit Märtha von Schweden, 1929 

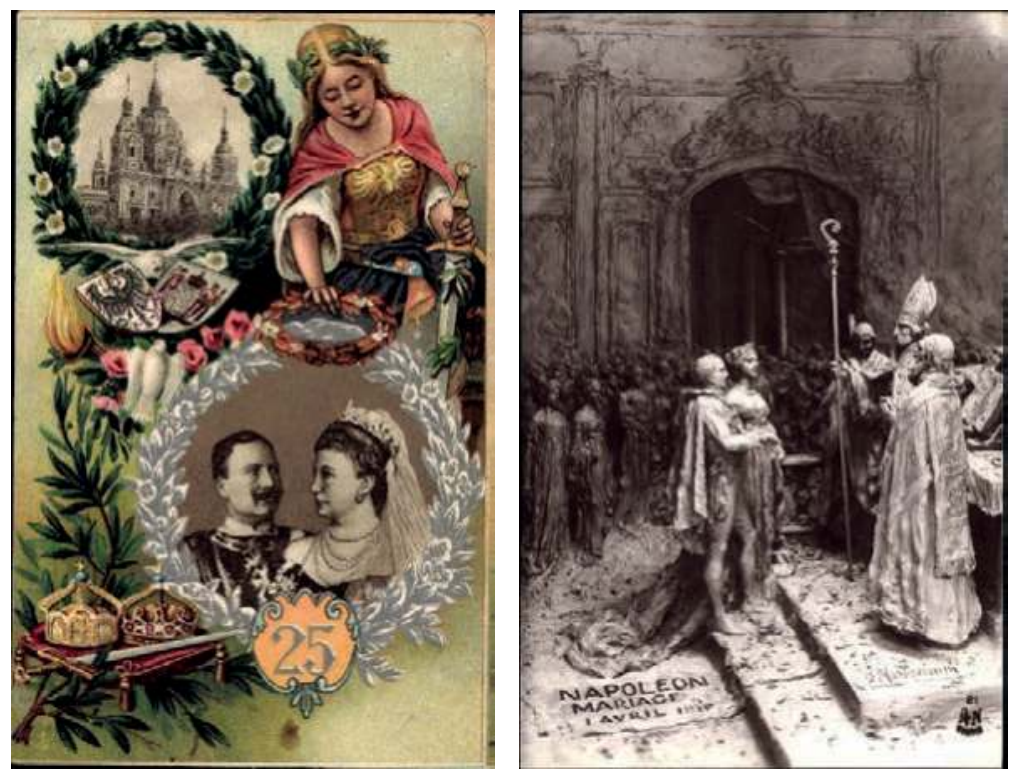

Abbildung 3-4: Ansichtskarte anlässlich der Silberhochzeit des Deutschen Kaiserpaares (1906) und Napoleons zweite Hochzeit mit Marie-Louise von Österreich 1810 (um 1900).
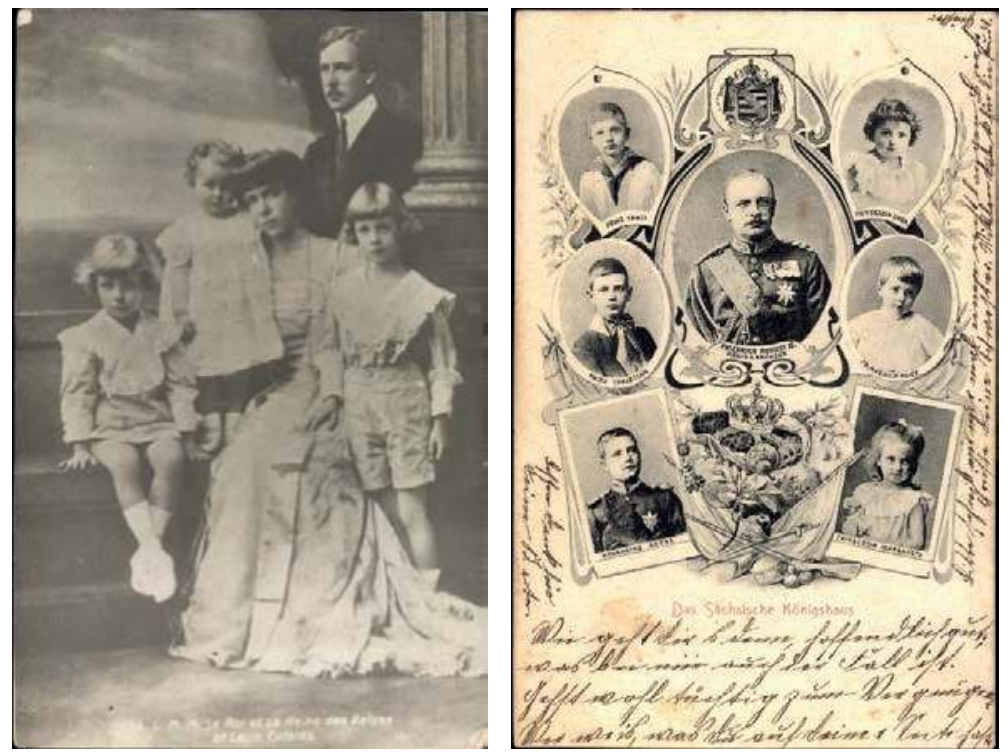

Abbildung 5-6: König Albert I. mit seiner Familie (um 1910) und König Friedrich August III. von Sachsen, mit seinen Kindern (um 1905). 

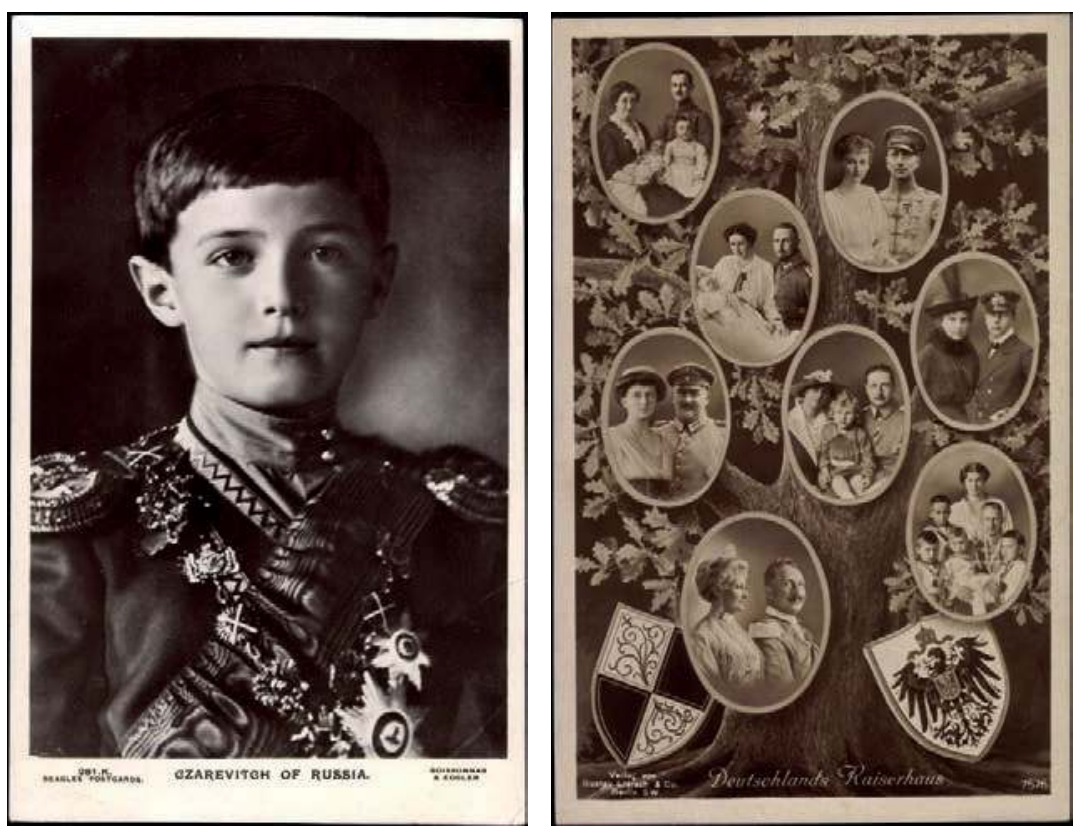

Abbildung 7-8: Zarewitsch Alexei Nikolajewitsch Romanow (um 1914) und »Deutschlands Kaiserhaus« (um 1914).

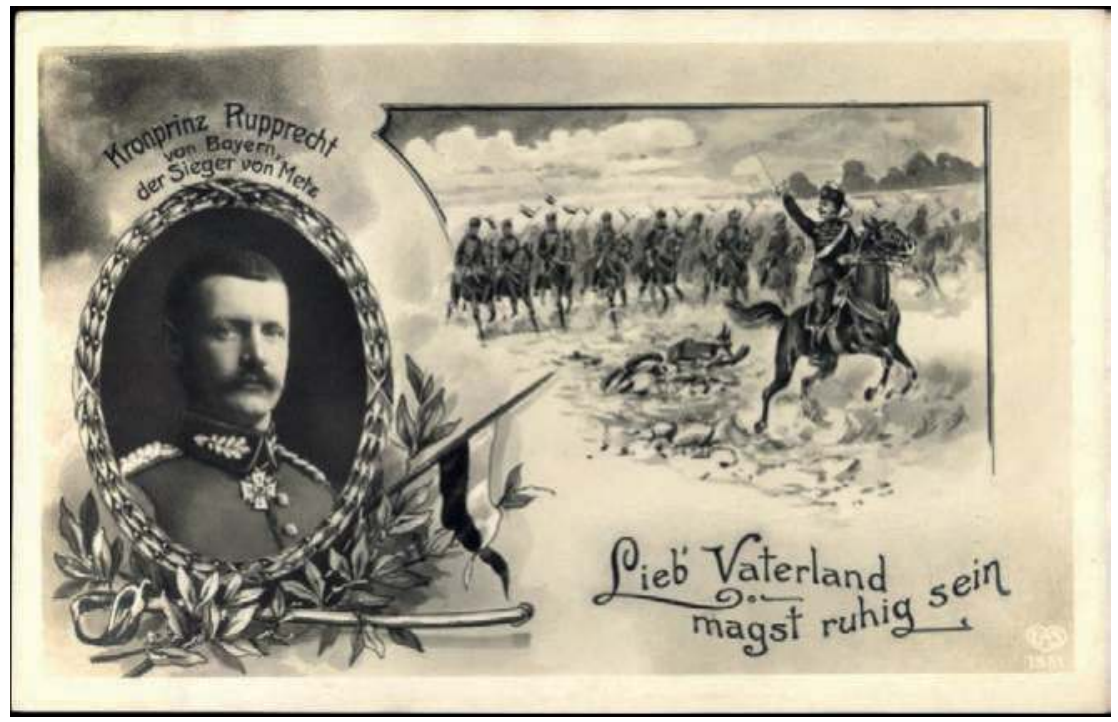

Abbildung 9: „Kronprinz Rupprecht von Bayern, der Sieger von Metz« (1914). 

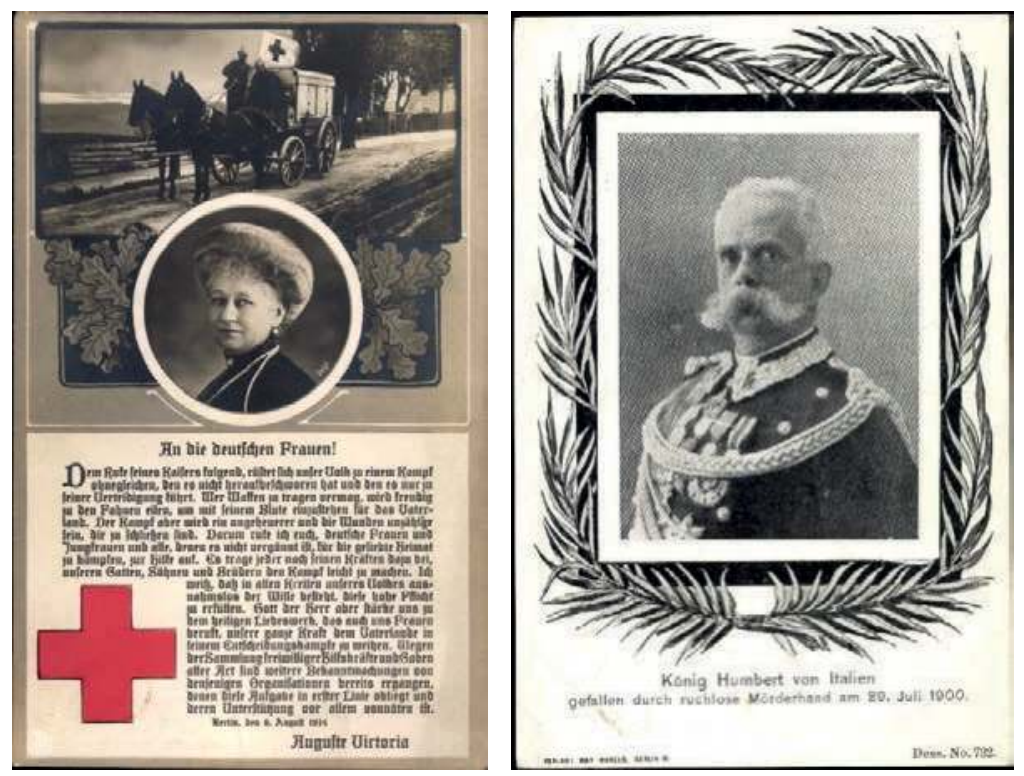

Abbildung 10-11: »An die deutschen Frauen!« (um 1914) und Memoriaansichtskarte anlässlich der Ermordung König Humberts (1900).
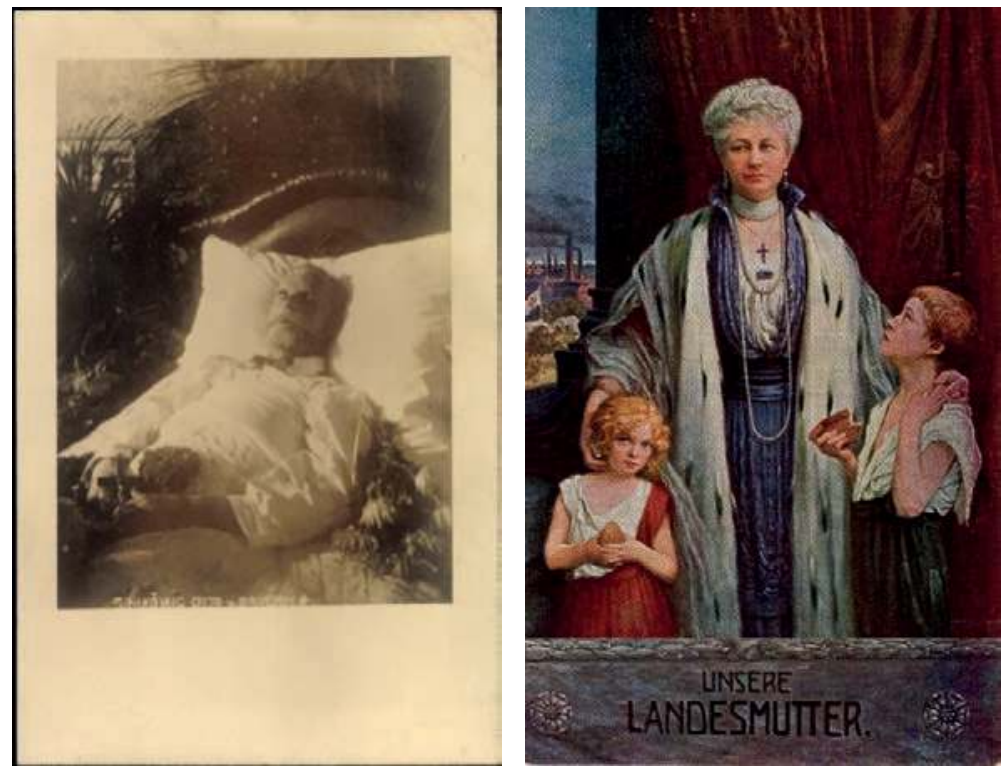

Abbildung 12-13: Aufbahrung König Ottos von Bayern (1916) und »Unsere Landesmutter« (um 1910). 

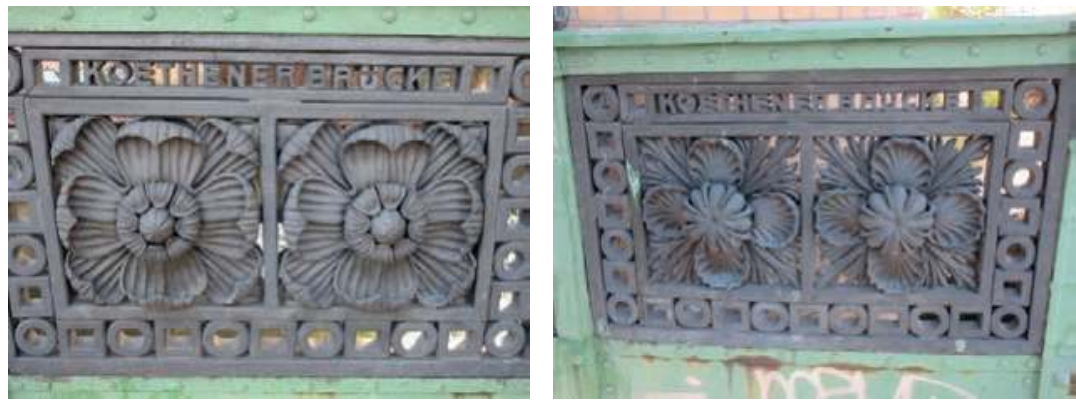

Abbildung 14-15: Detailfotografien der Arbeiten an der Köthener Brücke in Berlin-Kreuzberg und Kaiserin Auguste (um 1913).
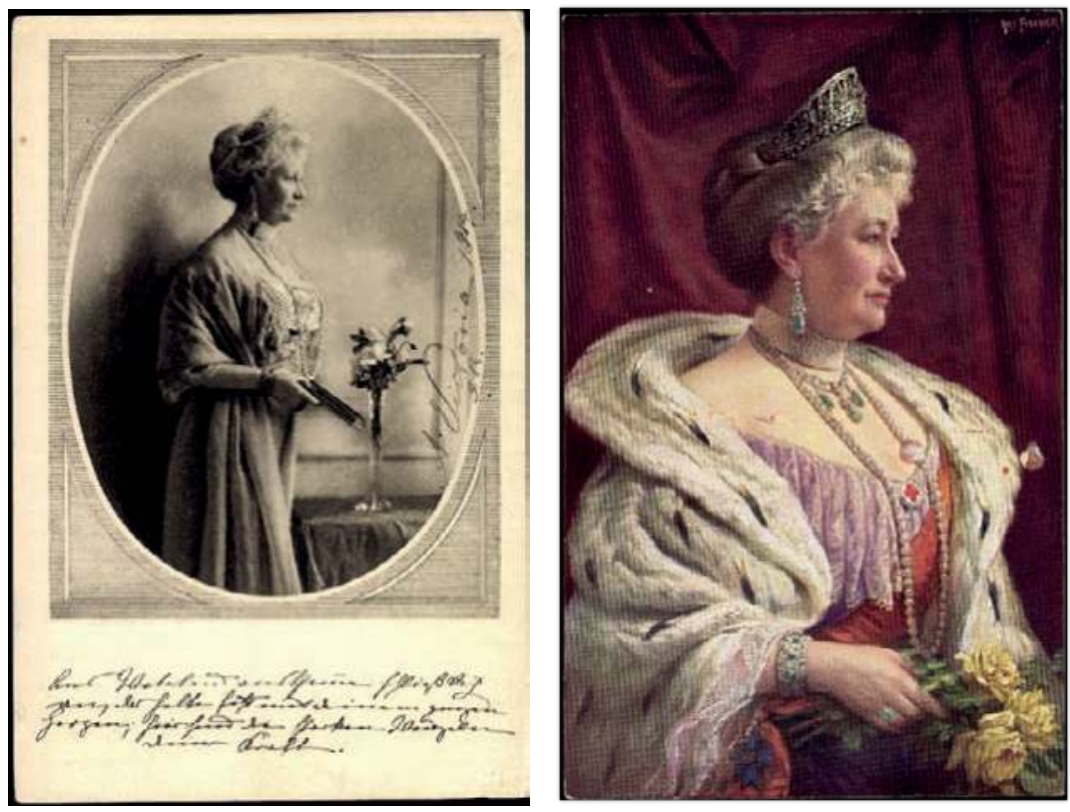

Abbildung 16-17: Kaiserin Auguste (um 1913). 

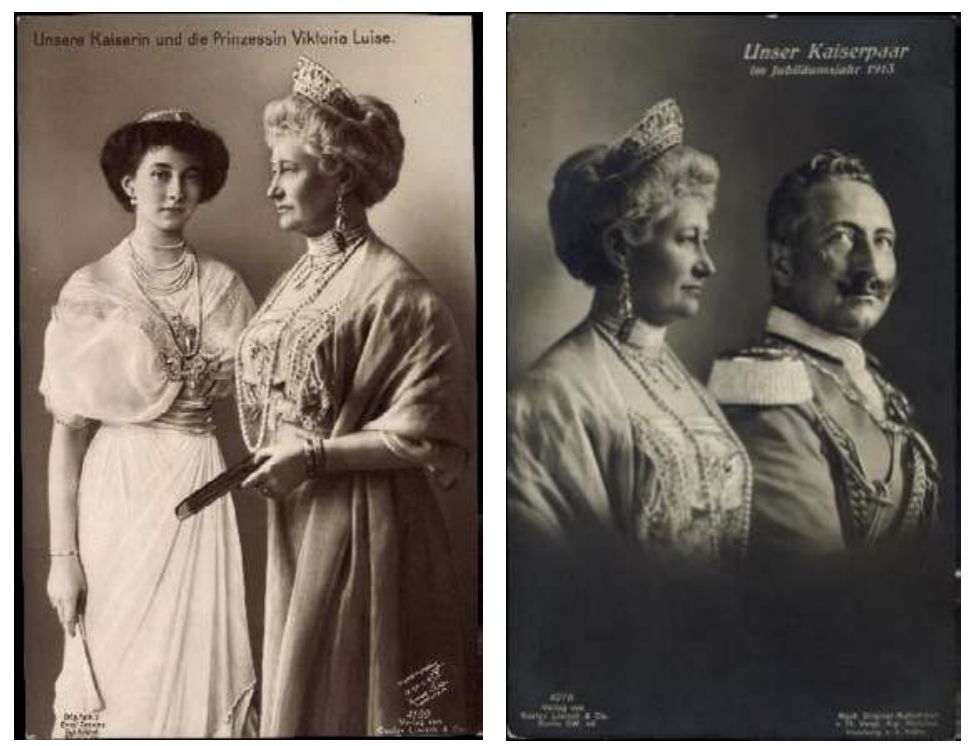

Abbildung 18-19: Kaiserin Auguste mit Prinzessin Viktoria Luise (um 1913) und Kaiserin Auguste mit Kaiser Wilhelm II (um 1913).

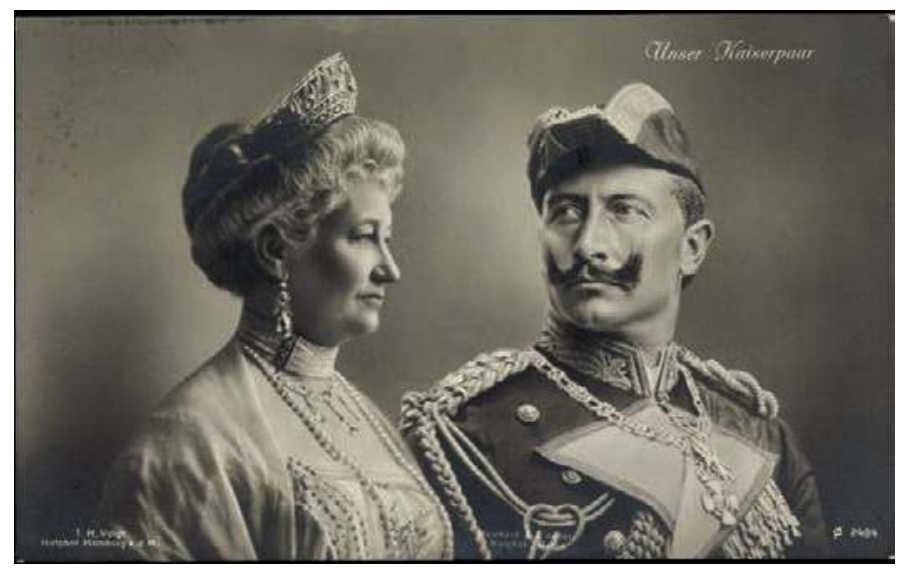

Abbildung 20: Kaiserin Auguste mit Kaiser Wilhelm II (um 1913) 

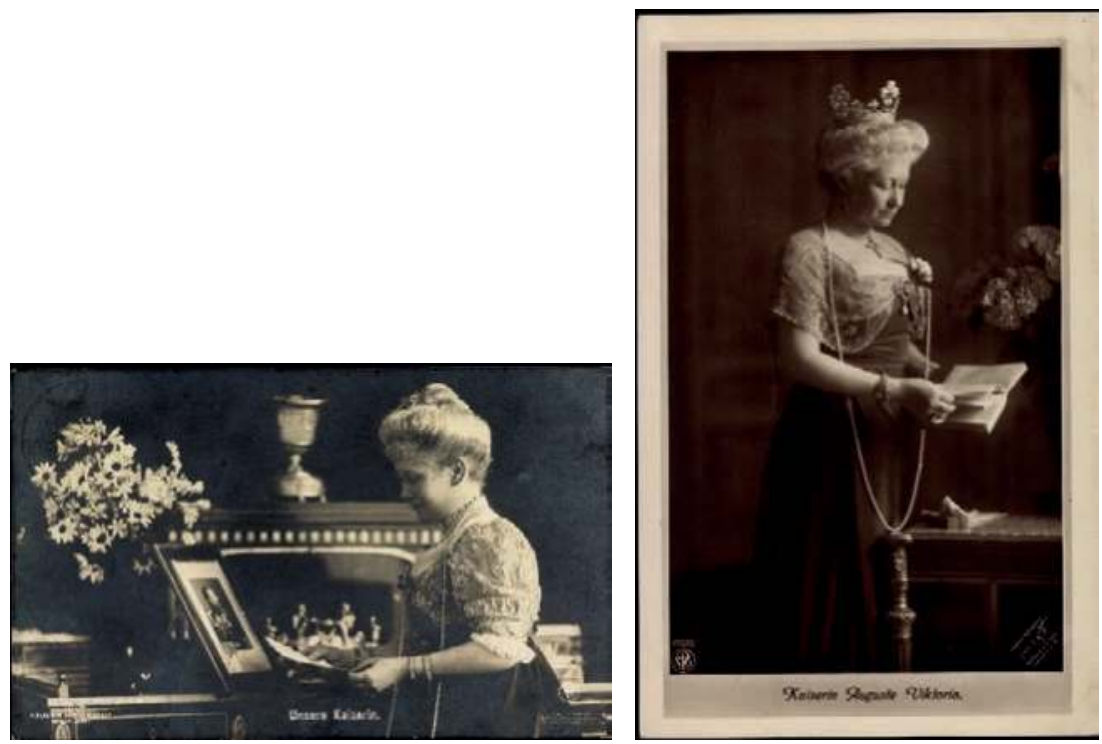

Abbildung 21-22: Kaiserin Auguste (um 1910).und Kaiserin Auguste (um 1910). 
Open-Access-Publikation im Sinne der CC-Lizenz BY-NC-ND 4.0 (C) 2021, Vandenhoeck \& Ruprecht GmbH \& Co. KG, Göttingen ISBN Print: 9783847112280 - ISBN E-Lib: 9783737012287 


\section{Abbildungsnachweise}

\section{Christoph Borbach}

Abb. 1: Vanessa Couchman: The Pneumatic Postal Service of Paris., online unter:

https://vanessacouchmanwriter.com/2019/07/02/the-pneumatic-postal-service-of-paris (letzter Zugriff: 15.07.2020)

Abb. 2: Ingmar Arnold: Luft-Züge. Die Geschichte der Rohrpost, Berlin 2016, S. 128

Abb. 3: Charles Bontemps: Les Systèmes Télégraphiques. Aériens - Électriques - Pneumatiques,

Paris 1876, Pl. IX

\section{Christian Methfessel}

Digitale Kartenbeilage 1: Sammlung Perthes: SPA-K0-227. Online verfügbar unter:

http://vandenhoeck-ruprecht-verlage.com/PWZ2 (unter Downloads); Lizenz: CC BY-SA 4.0

International

\section{Stefan Geissler}

Abb. 1: Wreck Book 1820, Guildhall Library MS31641_001, S. 43

Abb. 2: Lloyd's List, No. 17,700, Monday, January 9th 1871, S. 11

Abb. 3: Committee of Lloyd's, London 1821, S. 21

Abb. 4: Privates Foto by Michael Offerman, Universität Bern, 2018

\section{Reiner Prass}

Abb. 1: Sammlung Perthes: SPA ARCH PGM 195, Bl. 65

Abb. 2: Sammlung Perthes: SPA ARCH PGM 62, Bl. 1118

Abb. 3: Sammlung Perthes: SPK-10-IV.D C-15

Abb. 4: Sammlung Perthes: SPA ARCH PGM 354, Bl. 123

Digitale Kartenbeilage 2: Sammlung Perthes: SPK-10-IV.D C-16. Online verfügbar unter: http://vandenhoeck-ruprecht-verlage.com/PWZ2 (unter Downloads); Lizenz: CC BY-SA 4.0 International 


\section{Maksim Fomenko}

Abb. 1: https://www.stihi.ru

Abb. 2: https://topwar.ru

Abb. 3: https://fotosky.ru

Abb. 4: http://livinghistory.ru

Abb. 5: http://smolbattle.ru

Abb. 6: https://warspot.ru

\section{Heinz Wewer}

Abb. 1, 4, 6, 7, 9-11: Berlin, Sammlung Heinz Wewer

Abb. 2: Ravensbrück, Archiv der Mahn- und Gedenkstätte, V3706F2 02

Abb. 3: Ravensbrück, Archiv der Mahn- und Gedenkstätte, KL 31-89 01

Abb. 5, 12, 16-18: Berlin, Sammlung Wolfgang Haney

Abb. 8: Dachau, Archiv der KZ-Gedenkstätte, A 2555/38252/14/1

Abb. 13: Hailfingen/Tailfingen, KZ-Gedenkstätte

Abb. 14: Heidelberg, Dokumentations- und Kulturzentrum Deutscher Sinti und Roma

Abb. 15: Berlin, Sammlung Manfred Schulze

\section{Konstanze Soch}

Abb. 1: BStU, MfS, HA II, Fo, Nr. 314, Bild 12

Abb. 2: BStU, MfS, HA II, Fo, Nr. 314, Bild 4

Abb. 3: BStU, MfS, BV Lpz, Abt. VII, Fo, Nr. 1320, Bl. 1, Bild 9

Abb. 4: BStU, MfS, ZAIG, Nr. 10555, Bl. 12, Bild 44

Abb. 5: BStU, MfS, BV Lpz, Abt. KuSch, Fo, Nr. 739, Bild 7

\section{Amelie Dreecke}

Abb. 1-12, 16-22: Bildagentur arkivi

Abb. 13: Sammlung von Amelie Dreecke

Abb. 14-15: Fotografie von Amelie Dreec.ke (21.04.19) 